\title{
Investigation of the Linker Region of Coiled Coil SNARE-Analoga and Membrane Composition on Vesicle Fusion
}

\author{
Dissertation \\ for the award of the degree \\ "Doctor rerum naturalium" \\ of the Georg-August-Universität Göttingen \\ within the doctoral program chemistry \\ of the Georg-August University School of Science (GAUSS)
}

submitted by

Mike Christopher Groth

from Hannover

Göttingen 2020 


\section{Thesis Committee}

Prof. Dr. Ulf Diederichsen

Institute of Organic and Biomolecular Chemistry, Georg-August Universität Göttingen

Prof. Dr. Claudia Steinem

Institute of Organic and Biomolecular Chemistry, Georg-August Universität Göttingen

\section{Members of the Examination Board}

Reviewer:

Second Reviewer:

\author{
Prof. Dr. Ulf Diederichsen \\ Institute of Organic and Biomolecular Chemistry, University \\ of Göttingen
}

Prof. Dr. Claudia Steinem

Institute of Organic and Biomolecular Chemistry, University

of Göttingen

\section{Further Members of the Examination Board}

\section{Prof. Dr. Kai Tittmann}

Department of Molecular Enzymology, University of Göttingen

Prof. Dr. Michael Meinecke

Department of Cellular Biochemistry, University Medical Center Göttingen

Prof. Dr. Jörg Enderlein

III. Physical Institute, University of Göttingen

Dr. Holm Frauendorf

Institute of Organic and Biomolecular Chemistry, University of Göttingen

Date of the oral examination: $11^{\text {th }}$ January 2021 
The following work was carried out under the supervision of Prof. Dr. Ulf Diederichsen at the Institute of Organic and Biomolecular Chemistry of the Georg-August-University of Göttingen between January 2017 and November 2020.

This thesis was supported by the Deutsche Forschungsgemeinschaft in the context of the collaborative research center 803 (SFB 803, Functionality controlled by organization in and between membranes).

\section{Declaration of Authorship}

I declare that I prepared the doctoral thesis entitled "Investigation of the Linker Region of Coiled Coil SNARE-Analoga and Membrane Composition on Vesicle Fusion" on my own and with no other sources and aids than quoted.

Göttingen, 27.11.2020

Mike Christopher Groth 



\section{Contents}

1 Introduction.........................................................................................................................

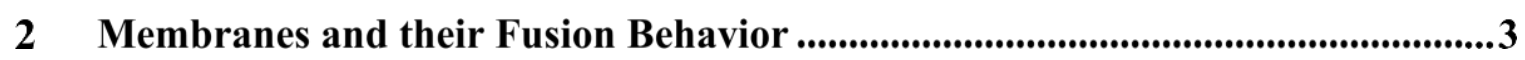

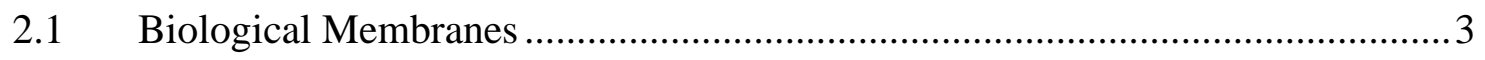

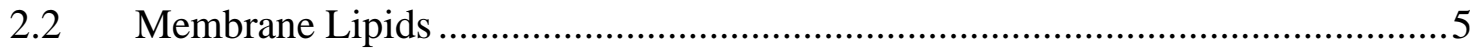

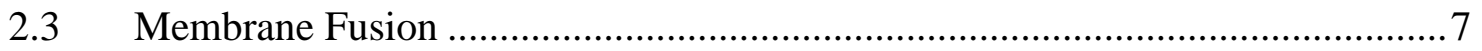

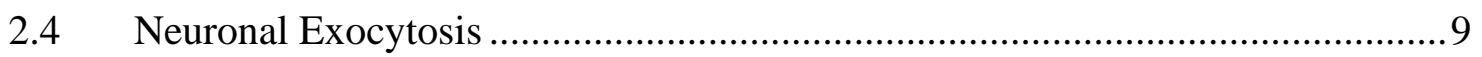

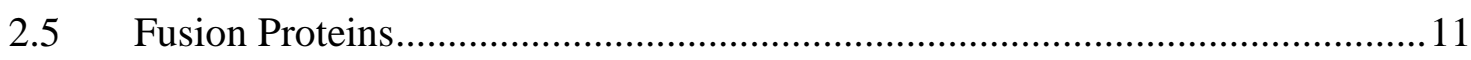

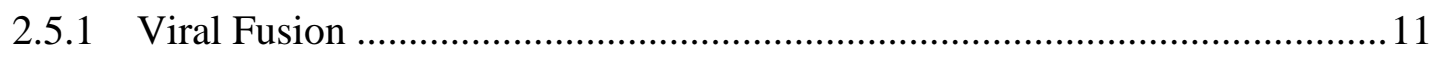

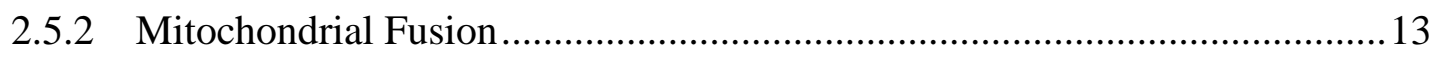

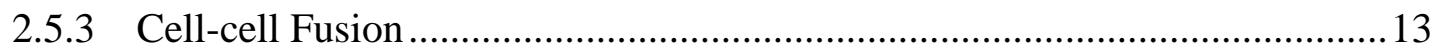

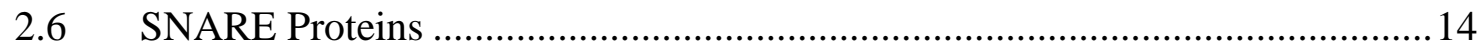

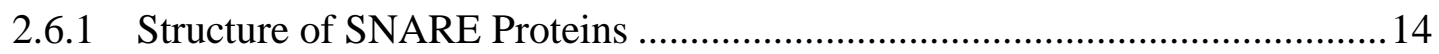

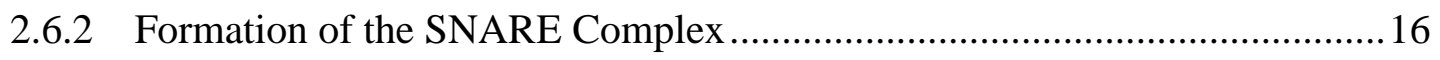

2.6.3 Regulation of the SNARE Complex Formation .......................................... 18

2.6.4 Role of the Linker in SNARE mediated Fusion ..........................................20

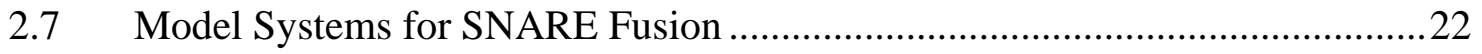

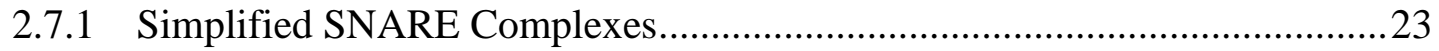

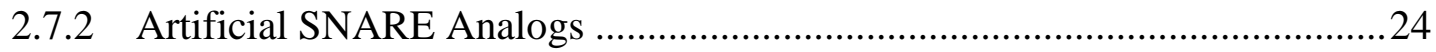

2.7.3 E3/K3-PEG-Lipid Model System ........................................................... 27

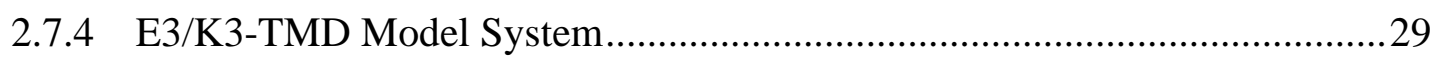

3 Detection of Liposome Fusion Events ........................................................................33

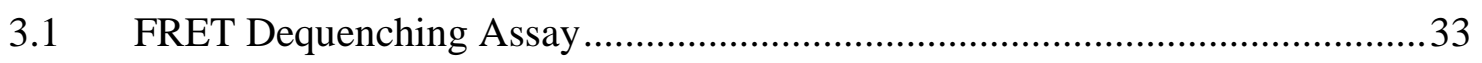

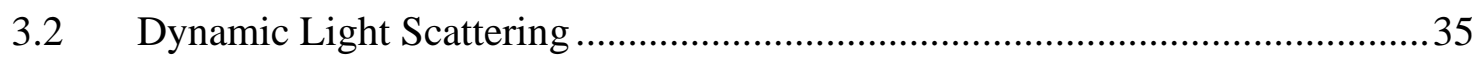

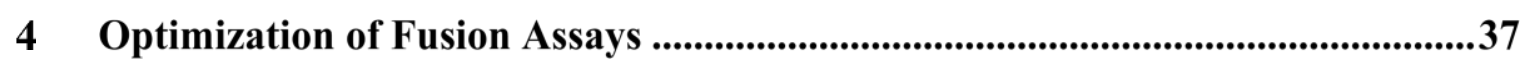

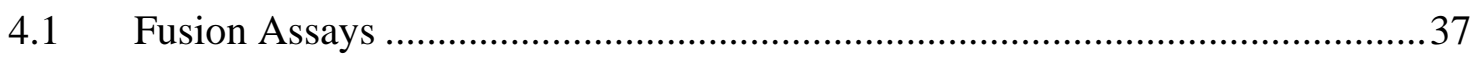

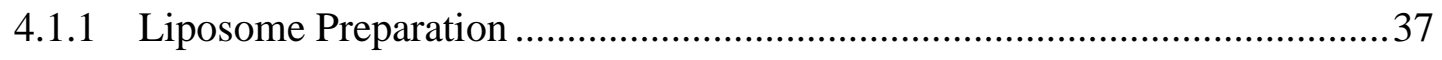

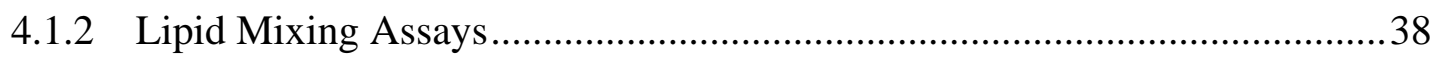

4.1.3 Dynamic Light Scattering Experiments ........................................................ 39 


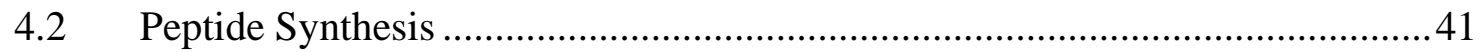

4.3 Enhancement of FRET Dequenching Assays ................................................43

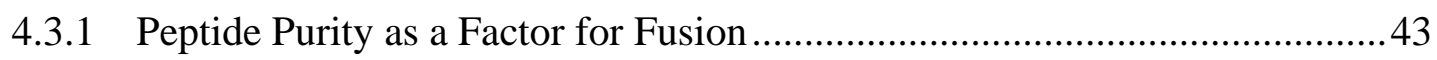

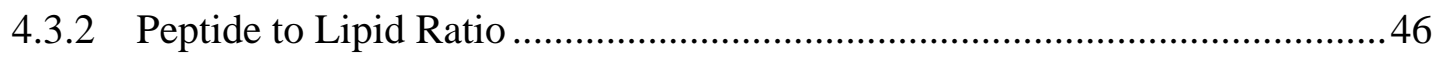

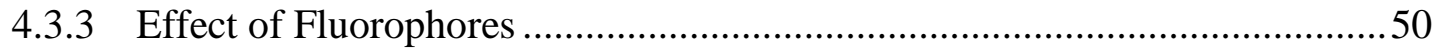

4.3.4 Assessment of Control Experiments ..........................................................53

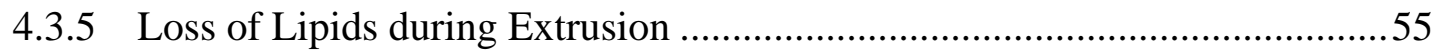

5 Modifications of the E3/K3-TMD Model System......................................................57

$5.1 \quad$ Effect of the Linker Length on Fusion Efficiency ........................................57

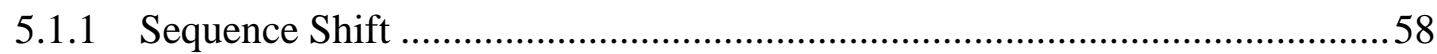

5.2 Aromatic Residues in the Linker Domains ....................................................6 60

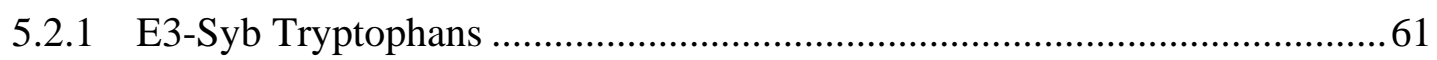

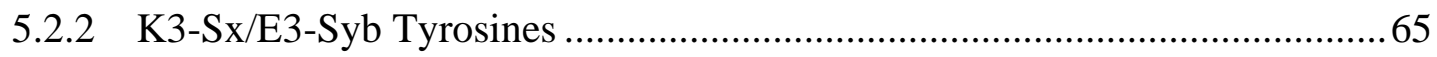

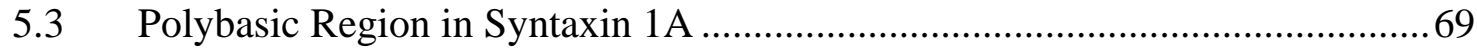

5.3.1 Role of the Arginine Domain in the Linker of Syntaxin 1A..........................71

5.3.2 Lysines in the Linker of Syntaxin 1A ....................................................... 74

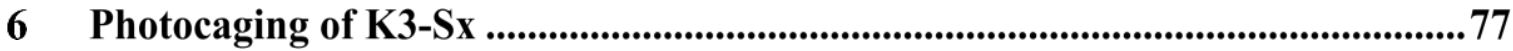

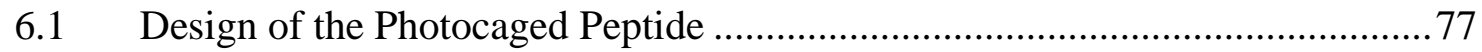

6.2 Synthesis of the Photocaged Peptide .................................................................. 81

6.3 FRET Dequenching Assays of DEACM caged Peptide .................................. 83

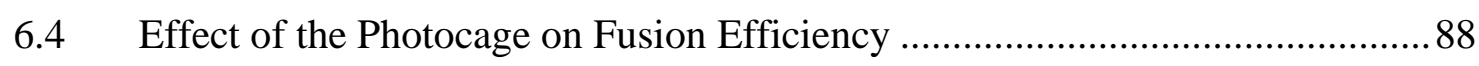

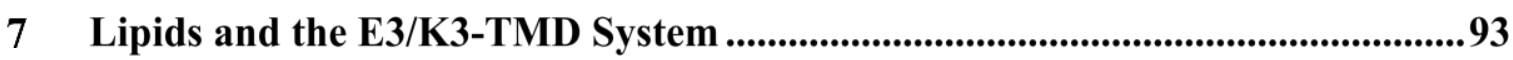

7.1 Effect of the Lipid Composition on the E3/K3-TMD System..........................93

7.2 Incorporation of DOPS in the Vesicle Membrane .......................................... 95

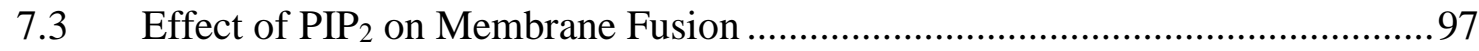

8 Conclusion and Outlook ...............................................................................................101

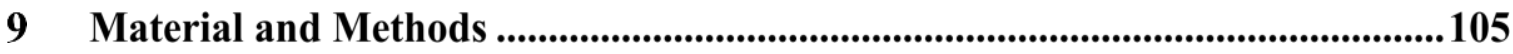

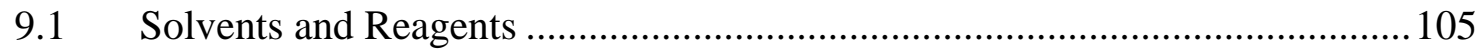

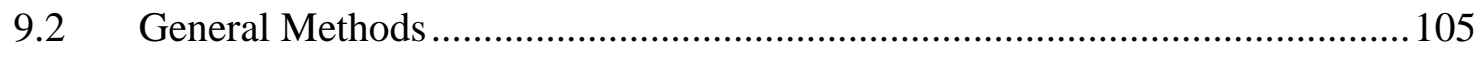




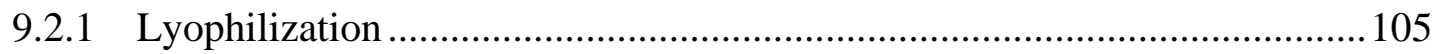

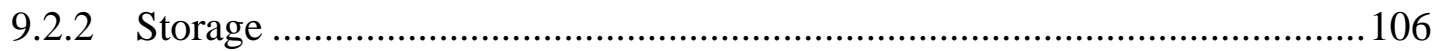

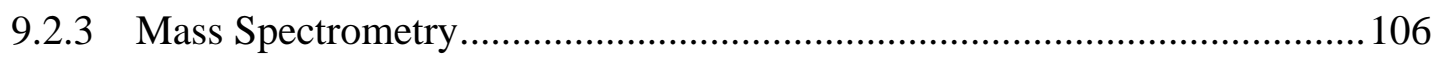

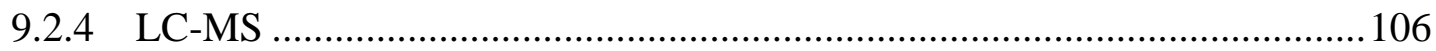

9.2.5 Nuclear Magnetic Resonance Spectroscopy ................................................ 106

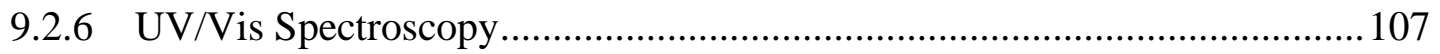

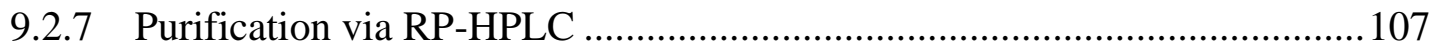

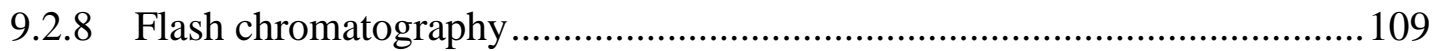

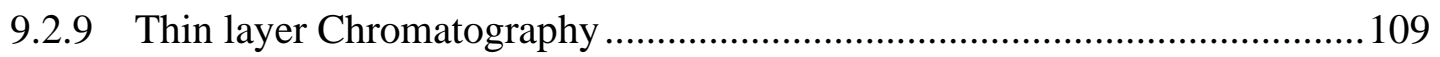

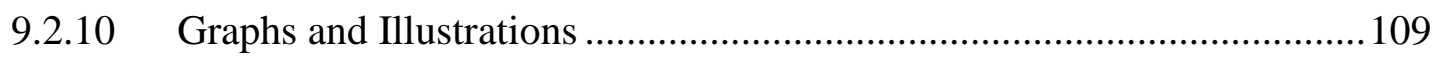

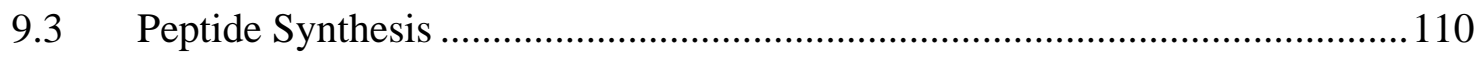

9.3.1 Solid Phase Peptide Synthesis (SPPS) …................................................... 110

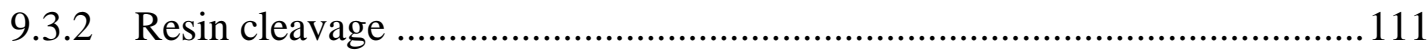

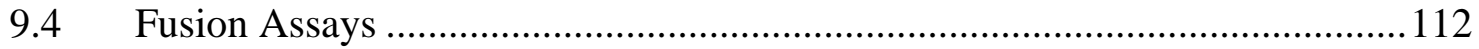

9.4.1 Lipid Film Preparation ............................................................................. 112

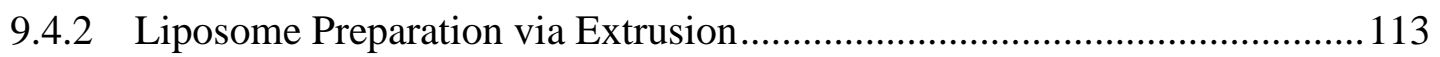

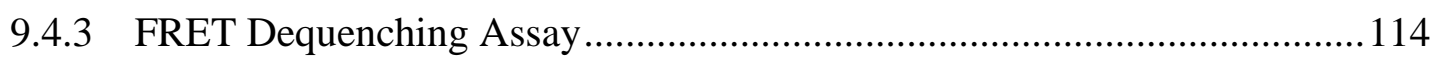

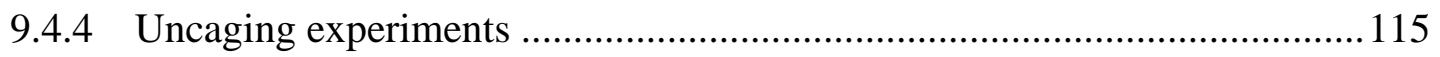

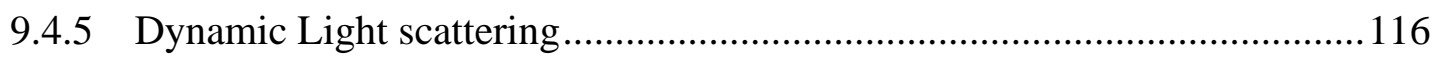

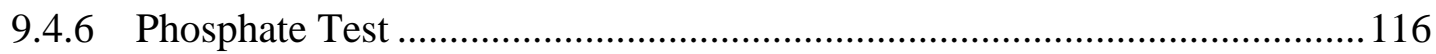

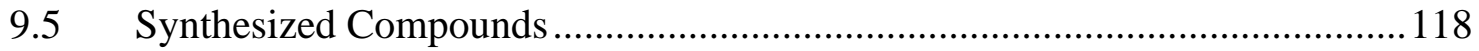

9.5.1 Synthesized Molecules................................................................................ 118

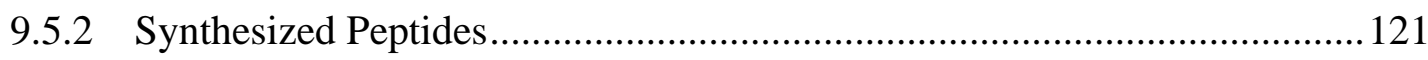

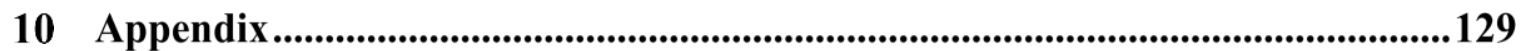

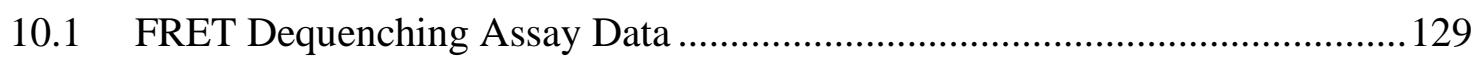

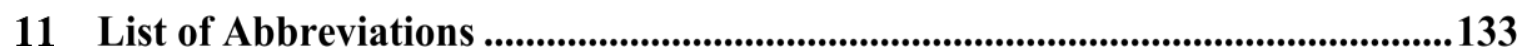

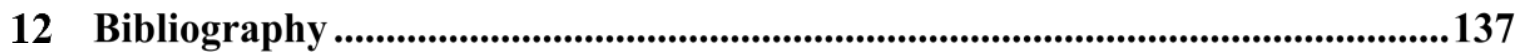





\section{List of Figures}

Figure 2.1: Schematic model of the different components located at cell membranes.....4

Figure 2.2: Illustration of membrane properties due to lipid diversity. ...........................6

Figure 2.3: Illustration of several fusion pathways of two lipid bilayers.........................

Figure 2.4: Illustration of neuronal signal transduction synaptic activity....................... 10

Figure 2.5: Schematic diagram of viral membrane fusion............................................ 12

Figure 2.6: Illustration of the structure of three neuronal SNARE proteins. ...................15

Figure 2.7: Illustration of the SNARE zippering mechanism...................................... 17

Figure 2.8: Schematic illustration of the recent presumed fusion cycle of synaptic

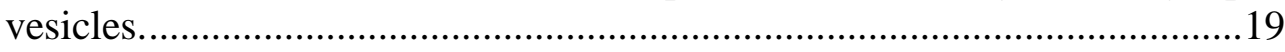

Figure 2.9: Illustration of the linkers of synaptobrevin 2 and syntaxin 1 A. ....................21

Figure 2.10: Illustration of the assembly pathway of SNAREs.....................................23

Figure 2.11: Illustration of the DNA based SNARE analog from STENGEL et al.............25

Figure 2.12: Illustration of the PNA-TMD model system by LYGINA et al.....................26

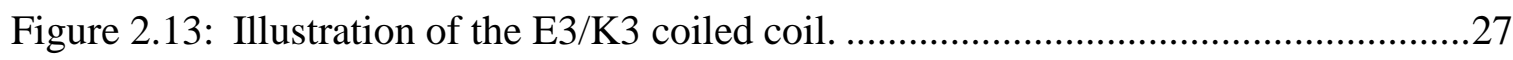

Figure 2.14: Schematic representation of the fusion behavior of LPE and LPK by DAUDEY

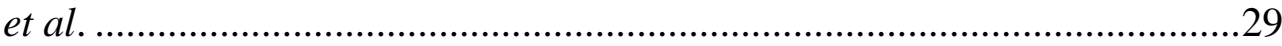

Figure 2.15: Illustration of the E3/K3-TMD system by MEYENBERG et al. ......................30

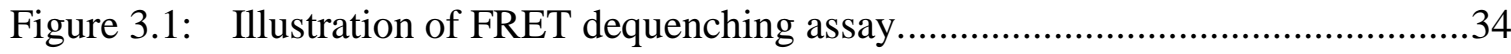

Figure 4.1: Exemplary DLS data of the three different vesicle populations...................40

Figure 4.2: UPLC chromatograms of a raw/unpurified peptide and the same peptide after HPLC purification. ..............................................................................44

Figure 4.3: FRET dequenching assay of the reference system with a peptide/lipid ratio of $1: 200$. 45

Figure 4.4: DLS data of the different used vesicle species........................................47

Figure 4.5: FRET dequenching assay comparison between different P/L ratios and their corresponding control.

Figure 4.6: DLS data comparison of vesicle species with different P/L ratios and different age.

Figure 4.7: FRET dequenching assays of different labeled vesicle species. .51

Figure 4.8: Comparison of FRET dequenching assay control measurements using different vesicle species. .53

Figure 5.1: Visualization of the shift in the sequence between natural protein and model system. .58

Figure 5.2: $\quad$ FRET dequenching assay of E3-Syb(RKY) and K3-Sx(KYQSK). .59

Figure 5.3: Illustration of the aromatic amino acids in the linkers of synaptobrevin 2 and syntaxin $1 \mathrm{~A}$ 
Figure 5.4: Comparison of different E3-Syb modified peptides. .63

Figure 5.5: Linker and TMD of synaptobrevin 2 and syntaxin 1A in the post fusion cis configuration. .66

Figure 5.6: FRET dequenching assay of tyrosine substituted peptides. .67

Figure 5.7: Exemplary DLS data of one measurement with E3-Syb(RKA) (blue) and $\mathrm{K} 3-\mathrm{Sx}(\mathrm{KAQSK})$ (red) containing vesicles.

Figure 5.8: Linkers and TMDs of synaptobrevin 2 and syntaxin $1 \mathrm{~A}$ in the post fusion $c$ is configuration. .70

Figure 5.9: FRET dequenching assay of the modified K3-Sx(QSKAAAK) peptide. ....71

Figure 5.10: FRET dequenching assay of the modified K3-Sx(QSAARRA) peptide......74

Figure 6.1: Schematic depiction of the experiment employed for the investigation of the fusion process of the E3/K3-TMD model system.

Figure 6.2: Illustration of a composition of the caged linker region of syntaxin 1A with DEACM.

Figure 6.3: Marked lysines in the linkers and TMDs of synaptobrevin 2 and syntaxin 1A in the post fusion cis-configuration. .80

Figure 6.4: Synthesis route of the photo cage Fmoc-L-Lys-(DEACM)-OH. .82

Figure 6.5: $\quad$ FRET dequenching assay with the caged K3-Sx peptide. .84

Figure 6.6: Fluorescence emission spectrum with excitation at $460 \mathrm{~nm}$ of E3-Syb vesicles.

Figure 6.7: Fluorescence emission spectrum with excitation at $460 \mathrm{~nm}$ of K3-Sx(QSXARRX) vesicles with different amounts of laser exposure....

Figure 6.8: UPLC chromatograms of $\mathrm{K} 3-\mathrm{Sx}(\mathrm{QSXARRX})$ after different amounts of laser exposure.

Figure 6.9: Comparison of different K3-Sx vesicle species in FRET dequenching assays.

Figure 7.1: FRET dequenching assay with the reference peptides E3-Syb(RKY) and K3$\mathrm{Sx}(\mathrm{QSK})$ in varying membrane lipid compositions.

Figure 7.2: FRET dequenching assay of vesicles containing DOPS. .95

Figure 7.3: Fusion curves obtained in FRET dequenching assays with different amounts of PIP

Figure 7.4: Fusion curves obtained in FRET dequenching assays with different amounts of $\mathrm{PIP}_{2}$. .98 


\section{List of Tables}

Table 4.1: Peptides used for the optimization process.................................................43

Table 4.2: Sizes of differently labeled vesicle species obtained via DLS....................52

Table 4.3: Vesicle sizes of liposomes from varying control experiments obtained via

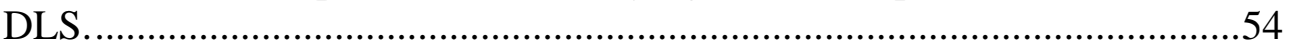

Table 4.4: Results of phosphate tests for different vesicle species...............................56

Table 5.1: Comparison of the peptide sequences for the experiments targeting sequence shift.

Table 5.2: Vesicle sizes of liposomes from experiments targeting the linker length of the model system.

Table 5.3: Peptide names and sequences synthesized for the determination of the role of the WW-domain in synaptobrevin 2.

Table 5.4: DLS data of the vesicles used for experiments regarding the WW-domain of synaptobrevins linker.

Table 5.5: Peptide names and sequences with substituted tyrosine residues. .67

Table 5.6: DLS data of measurements performed for the investigation of the role of tyrosine in the linker.

Table 5.7: Peptide sequences of reference and modified K3-Sx peptides used for the investigation of the polybasic area in syntaxin. . .71

Table 5.8: DLS data of vesicle species used in the experiments regarding the arginine domain of syntaxin $1 \mathrm{~A}$

Table 5.9: DLS data of vesicle species used in the experiments regarding the lysines of the linker of syntaxin.

Table 6.1: Comparison between the reference K3-Sx peptide and the caged species sequence.

Table 6.2: DLS data of vesicle species used in the uncaging experiment. .85

Table 6.3: Sizes of vesicle species used in the uncaging experiments obtained via DLS. . .90

Table 7.1: DLS data of vesicle species with a lipid composition of

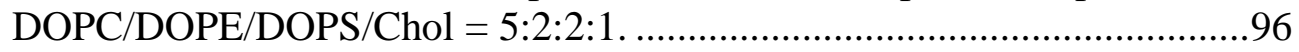

Table 7.2: Average of the recorded sizes of vesicle species used with varying $\mathrm{PIP}_{2}$ concentrations.

Table 9.1: Extinction coefficients used in this thesis. 107

Table 9.2: HPLC-Systems used for analysis and bulk purification of peptides. 108

Table 9.3: Columns used for peptide analysis and purification and the flow rates at which they were used.

Table 9.4: List of solvent systems used during LC peptide analysis and purification.109

Table 9.5: List of synthesizers used for automated microwave assisted peptide synthesis. 
Table 9.6: Used amino acids and concentrations of reagents for automated peptide synthesis on the Liberty Blue synthesizers. ...........................................110

Table 9.7: List of the different coupling methods used for peptide synthesis.............111

Table 9.8: List of the used lipid compositions in this thesis for the preparation of

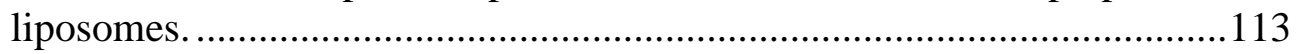

Table 9.9: Parameters applied in fluorescence measurements. .................................. 114 


\section{Introduction}

Communication between cells is a crucial process in multicellular organisms. One prominent communication pathway is the signal transduction between nerve cells. Here, an electrical impulse is translated into a chemical signal, which transfers information from one neuron to another. An important part of this transmission is the neuronal exocytosis. Synaptic vesicles fuse with the presynaptic membrane and release the stored neurotransmitters into the synaptic cleft. ${ }^{[1,2]}$ During this process, two separate membranes have to overcome an energy barrier to facilitate fusion. This complex task can be achieved by the evolutionary conserved SNARE (soluble $N$-ethylmaleimide-sensitive factor attachment protein receptor) family. ${ }^{[3-5]}$ The vesicle membrane contains synaptobrevin 2 , the acceptor membrane syntaxin $1 \mathrm{~A}$ and the $25 \mathrm{kDa}$ synaptosome-associated protein SNAP 25. ${ }^{[6]}$ The interaction between these proteins is thought to start $\mathrm{N}$-terminally and proceed in a zipper-like manner toward the C-termini. ${ }^{[7-9]}$ The membranes are brought in close proximity so that they ultimately fuse together. The strong interaction between the proteins, which is needed for the mechanism, is a result of the formation of a four-helical bundle. ${ }^{[10,11]}$ Each of the involved proteins contributes its motif to form this structure. In case of synaptobrevin 2 and syntaxin 1A, the motif is attached via a small flexible linker sequence to a transmembrane domain (TMD) which anchors the protein into the membrane. ${ }^{[12,13]}$ Since the discovery of the SNAREs in the late 1980s, the protein family has been studied extensively. ${ }^{[14-16]}$ Different synthetic models have been developed to mimic the function of the SNAREs to even further improve the understanding of the underlying mechanism. ${ }^{[17-20]}$

One of these SNARE mimetics consists of a dimeric peptide system, which is designed to keep the main three domains as close to the natural system as possible. The linker and TMD are the native sequences of synaptobrevin $2(\mathrm{Syb})$ and syntaxin $1 \mathrm{~A}(\mathrm{Sx})$. The natural parts are attached to an artificial motif which consists of the coiled coil pair E3 and K3. ${ }^{[21]}$ Initially, this system was developed to be synthesized via solid phase peptide synthesis (SPPS) and to mimic the zippering mechanism of the natural SNARE complex. ${ }^{[22]}$ 
Furthermore, the natural peptidic backbone was kept to reduce unnatural structural changes. The pair of E3-Syb and K3-Sx peptides has already been verified to be capable of fusing large unilamellar vesicles (LUVs) in bulk fusion assays. ${ }^{[22]}$ One of the advantages of this system is its peptidic character which can be modified easily and can therefore be used to address specific questions regarding the fusion mechanism or peptide structure.

In fact, one of the objectives of this work is to investigate the fusion mechanism of the E3/K3-TMD model system and to connect the results to the natural SNARE fusion process. This is achieved by precisely stopping the fusion process after the coiled coil formation of the motifs and starting it again after a specific trigger. To accomplish this, a photocleavable protecting group was introduced into the linker of one of the peptides. Using Förster resonance energy transfer (FRET) based bulk fusion assays the inhibitory effect of the group was tested. For this purpose the position of the group in the peptide has been evaluated via the $\mathrm{x}$-ray data of the natural SNARE complex. ${ }^{[23]}$ Additionally, the predetermined positions have been investigated regarding their effect on fusion efficiency of the system. Peptide variants with substituted amino acids have been synthesized and tested in this regard.

Another focus of this work targets a deeper understanding of the role of the linker sequence in the fusion mechanism. To achieve this, the E3/K3-TMD systems sequences are selectively modified and the resulting changes of vesicle sizes and fusion behavior are monitored. The modifications are aimed at three properties of the linker. First, the effect of the linker length is tested. Here, the particular connection between the artificial motif and natural linker is of interested. Second, the charge of the polybasic area in syntaxins linker, which has been investigated by research groups for years. ${ }^{[24-26]}$ In this regard, the interaction with charged lipids was also investigated in this work. Especially PIP $_{2}$ (phosphatidyl 4,5-bisphosphate) is known for its interactions with syntaxins linker. ${ }^{[27,28]}$ Third, a ring of aromatic amino acids is believed to be responsible for the insertion depth of adjacent lysines, ${ }^{[29]}$ the stiffness of synaptobrevins linker, ${ }^{[23]}$ and plays a role in the fusion process itself. ${ }^{[30]}$ To test whether these amino acids are important for the E3/K3-TMD model systems ability to fuse vesicles, alterations in the peptide sequence have been monitored via FRET assays and DLS measurements. 


\section{Membranes and their Fusion Behavior}

This chapter focusses on the properties of biological membranes and on proteins which have the ability to fuse two separate lipid bilayers. Chapter 2.1 and 2.2 give an introduction in today's knowledge about biological membranes and their main building block lipids. The next two chapters (2.3 and 2.4) deal with membrane fusion and describe the neuronal exocytosis pathway. Sections 2.5 and 2.6 present proteins which are capable of performing the task of fusing separate lipid bilayers in nature. Finally, chapter 2.7 is dedicated to model systems, which are developed to mimic SNARE protein structure and/or imitate their fusion mechanism.

\subsection{Biological Membranes}

Biological membranes are an essential structure for cells and cell organelles to maintain their functional capabilities. ${ }^{[31]}$ Many different processes vital for life are occurring in or at membranes. The reason for this is the high number of different lipids, proteins, sugars and other molecules and cell organelles which are associated to the membrane (see Figure 2.1). The structural foundation of biological membranes are lipids (see chapter 2.2). ${ }^{[32]}$ These molecules have an amphipathic character due to their polar head groups and hydrophobic acyl tail chains and are therefore prone to form lipid bilayers in an aqueous environment. ${ }^{[33]}$ The driving force of this behavior is the hydrophobic interaction between the acyl chains. ${ }^{[34]}$

A key feature of these membranes is the separation of different cells and compartments. ${ }^{[35]}$ This opens up the possibility for diverse biochemical reaction environments and specialized domains within a cell. These domains provide a foundation for proteins and other molecules for important cellular tasks like proton gradient generation or signaling cascades. ${ }^{[36]}$ The bilayer can also act as a barrier to protect the cell against toxins or oxidation, as well as maintain an electrochemical gradient which is important for multiple other cell activities. ${ }^{[37]}$ 
Membrane proteins are responsible for many processes such as enzymatic activity, particle transport across the membrane, communication between cells and signal transduction. ${ }^{[38]}$ Classification of these proteins can be done via the type of attachment to the membrane. Integral proteins are embedded into the lipid bilayer via specific protein structures. They have been identified to contain $\alpha$-helical and/or $\beta$-barrel structures, which contain hydrophobic domains to stay inside the bilayer. ${ }^{[39,40]}$ Peripheral membrane proteins, on the other hand, are attached to the outside of the membrane. The proteins are mostly acting in the lipid-water interface and are fully water soluble. They can interact with the bilayer reversibly through electrostatic interactions, hydrophobic interactions or attachment to lipid anchors. $^{[41,42]}$

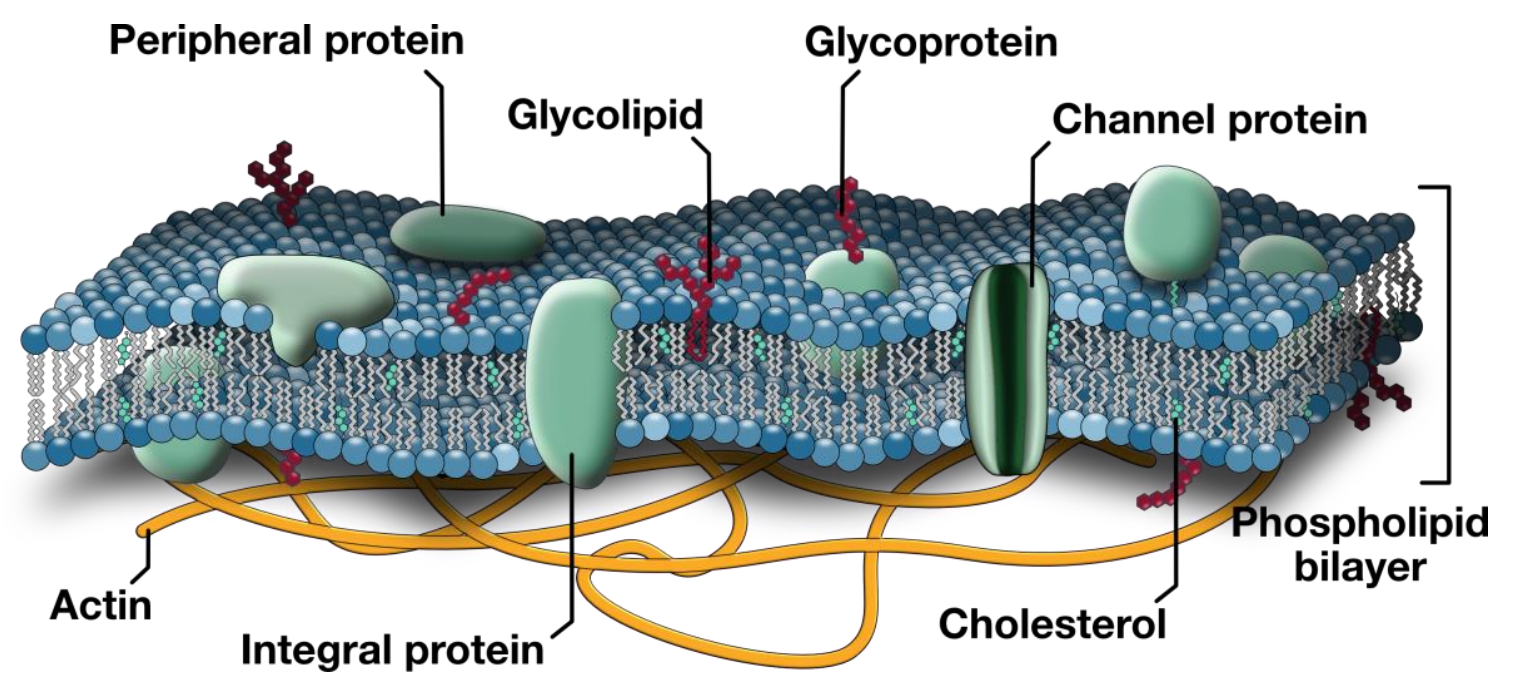

Figure 2.1: Schematic model of the different components located at cell membranes. The bilayer consists of various lipids (phospholipids, glycolipids, sphingolipids, etc.), proteins, sugars and other molecules. The components are usually not distributed evenly and are known to form specialized areas, where the local concentration of specific lipids or proteins is higher compared to the rest of the membrane.

Membranes are highly asymmetric regarding the lipid and protein composition between the two sides of the bilayer. ${ }^{[43,44]}$ Processes like ion pumping and signaling cascades have to be directional to serve a purpose for the cell. This asymmetric feature is vital for many cell activities and has for example been correlated with cell apoptosis. ${ }^{[45]}$

In 1972 SINGER and NICOLSON introduced the fluid mosaic model which contributed to a better understanding of lipid membranes and proteins at the time. ${ }^{[46]}$ Biological membranes are described as a two dimensional matrix, which is made up of a fluid bilayer of 
phospholipids in which mobile integral globular proteins are embedded. These proteins are distributed evenly throughout the fluid membrane but are also able to form aggregates at short ranges. The fluid mosaic model replaced the at that time prevalent unit membrane model and tri-layer model. ${ }^{[47-49]}$ As research progressed, the fluid mosaic model reached its limits and is not generally applicable for the description of membranes anymore. The model fails to describe lipid-lipid, lipid-protein, protein-protein, membrane-associated cytoskeletal and extracellular matrix interactions. ${ }^{[35]}$ Furthermore, observation of lateral membrane heterogeneity lead to the development of a new concept to explain these findings. The compartmentalization of the membrane was first discovered in the 1970s and led to the concept of lipid rafts. ${ }^{[50-53]}$ Rafts are defined as highly dynamic, heterogeneous sterol- and sphingolipid-enriched domains with a size of about $10-200 \mathrm{~nm} .{ }^{[54]}$ They are proposed to form relatively ordered domains which recruit other proteins and lipids to form functionally specialized membrane regions. ${ }^{[50]}$ However, as detection of these rafts is difficult and different methodologies often yield contradictory results, lipid rafts have yet to be observed in living cells. ${ }^{[52]}$ The ongoing controversy of this model sparked a debate about renaming these previous findings into "transient nanodomains" rather than rafts. ${ }^{[55]}$ Alongside this model, other alternatives discuss the organization inside the plasma membrane. ${ }^{[56-58]}$ Charge-mediated formation of clusters, lipid shells or areas confined by cortical actin skeleton may also compartmentalize the plasma membrane. ${ }^{[59]}$

\subsection{Membrane Lipids}

Lipids are the main building blocks of biological membranes and are essential for every organism. For example, about $50 \%$ of the human brains dry weight is accounted for by lipids. ${ }^{[60]}$ Plenty proteins rely on their amphoteric nature to form a suitable environment for protein activity. Understanding the properties and behavior of lipids is therefore crucial for the elucidation of membrane processes.

Membrane lipids are amphoteric molecules with a polar head group and a lipophilic acyl chain. Generally, these lipids can be categorized by their physicochemical properties. ${ }^{[61]}$ The chemical diversity can be achieved with different types of headgroups, backbones and acyl chains. Phosphatidylethanolamine, phosphatidylcholine and phosphatidylserine are prominent head groups. The backbone consists of either glycerol or sphingosine. The acyl chain can vary in length as well as in conformation due to possible double bonds. For 
phosphoglycerolipids a combination of two different acyl chains is also possible, increasing the number of available lipids. ${ }^{[32,61]}$ The majority of biological lipids can be classified into glycerophospholipids, sphingolipids and sterols. ${ }^{[32]}$ Another important characteristic of lipids is their interaction with each other. Due to the high number of lipids inside a lipid bilayer, compositional diversity expands the scope of application of membranes even further. Depending on the type or mixture of lipids in the membrane, several properties of the structure can be changed (see Figure 2.2). Membrane thickness, flexibility or even local charge can be modulated with different lipid compositions. As biological membranes consist of hundreds of different species (lipids and proteins) the complexity of these systems is immense. ${ }^{[62,63]}$

(a)

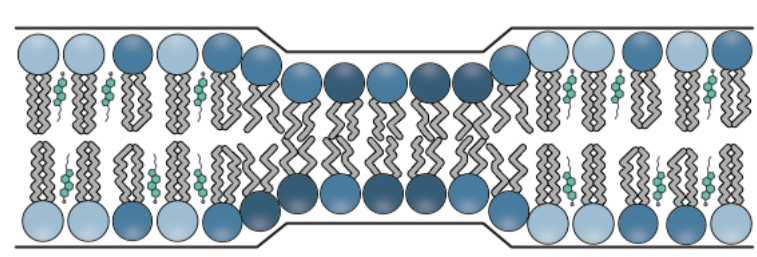

(b)

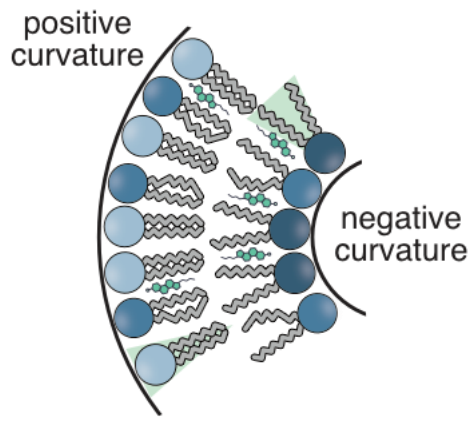

(c)

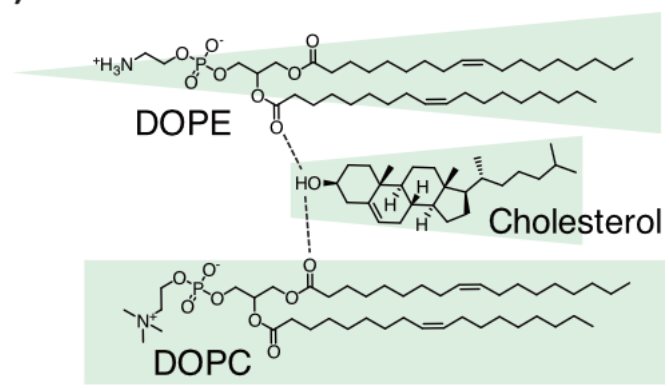

Figure 2.2: Illustration of membrane properties due to lipid diversity. (a) Membrane thickness is modulated by different lipids and cholesterol. (b) Depending on the curvature of the membrane, specific types of lipids are recruited. (c) DOPE as an example for a cone-shaped lipid and DOPC as a cylinder-shaped lipid. Location of cholesterol in between lipids is shown.

The fluidity and flexibility of lipid bilayers is a result of the interaction between the lipids and proteins present at this interface. ${ }^{[61]}$ Lipids have intrinsic shapes which are dependent on their hydrophobic acyl chains and hydrophilic head groups. Different geometric shapes of the lipids are possible. Cone shaped lipids are a result of headgroups having a smaller mean diameter compared to the acyl chains. Unsaturated phosphoethanolamine (PE) and phosphatidic acid (PA) lipids are cone shaped, whereas phosphatidylcholine (PC) headgroups tend to be cylindrical. The shape determines the side-by-side packing and 
therefore, the curvature of the monolayers. The thickness of the bilayer is also an important parameter which can determine the properties of a membrane. For example, the activity of many proteins is regulated via the thickness of the bilayer. ${ }^{[64]}$ The length of the acyl chains of lipids, the amount of cholesterol, as well as proteins itself can modify this property. ${ }^{[64,65]}$ Another important lipidic component are sterols, with the most prominent being cholesterol. ${ }^{[66]}$ Cholesterol modulates the bilayers fluidity, thickness and curvature, making it an essential component in mammalian cells. ${ }^{[65,67][65, \mathrm{ERL}}$ It increases the bilayer thickness by straightening the acyl chains of adjacent lipids and can be found preferably in negatively curved bilayers due to its intrinsic curvature. ${ }^{[65]}$ The molecule is positioned in between the acyl chains of the phospholipids with its hydroxyl group at the level of the ester groups. ${ }^{[68,69]}$ Furthermore, cholesterol is known to interact with many proteins e.g. SNARE proteins and HIV-1 gp41. ${ }^{[70,71]}$

\subsection{Membrane Fusion}

Fusion of biological membranes is the process in which two separate lipid bilayers overcome an energy barrier and merge together, forming one continuous bilayer. In most cases, this event also leads to mixing of the two contents which were enclosed by the two separate bilayers. There are two main mechanisms for the fusion process which can be separated into protein independent and protein dependent fusion. The mechanism for the former was first postulated by KOZLOV and MARKIN in 1983. ${ }^{[72]}$ The original theory describes a mechanism in which first a hemifusion step has to be passed before full fusion occurs (see Figure 2.3). Here, the two membranes are in close proximity when a point-likeprotrusion minimizes the hydration energy so that a hemifusion stalk can be formed $(1>2>3)$. In this stage, the outer membrane leaflets are mixed, whereas the inner leaflets stay separated. Continuing from the stalk, either a hemifusion diaphragm can be formed $(3>4),{ }^{[73]}$ or a direct fusion pore opening occurs $(3>5) .{ }^{[74]}$ Either way, the last step includes the formation of a fusion pore which enables the exchange of the aqueous contents between the formerly separated bilayers. The process of hemifusion stalk formation is generally agreed upon, as it has been observed with X-ray diffraction studies. ${ }^{[75,76]}$ Although the formation of a hemifusion diaphragm was experimentally observed, ${ }^{[77,78]}$ it is still debated whether the process can proceed to the pore formation from this stage. ${ }^{[79]}$ Since the first postulation, several improvements and additions on the "stalk model" have been made to 
describe the process more accurately. ${ }^{\left[{ }^{80-82]}\right.}$ Recently, the mechanism has been modified and adapted even further to accommodate several possible alternative fusion pathways. ${ }^{[83]}$ These new pathways are less symmetric than the original mechanism but originate from the hemifusion stalk.

During the first route $(3>6>7>8)$, the stalk grows linearly along a circular path to form an inverted micelle (6). From here, two pores need to be opened, with the first leading to the formation of a $\pi$-shaped hemifusion diaphragm (7). This diaphragm is similar to (4), however the lipids have been mixed during its formation.

In an alternative route, the fusion stalk opens transiently and forms the stalk-pore complex (9), which closes to form a hemifusion diaphragm before the fusion pore opening $(3>9>7>8) .{ }^{[84-86]}$ It is also currently debated, that the stalk-pore complex opens during the hemifusion diaphragma elongation $(3>4>9>7>8) .{ }^{[87]}$

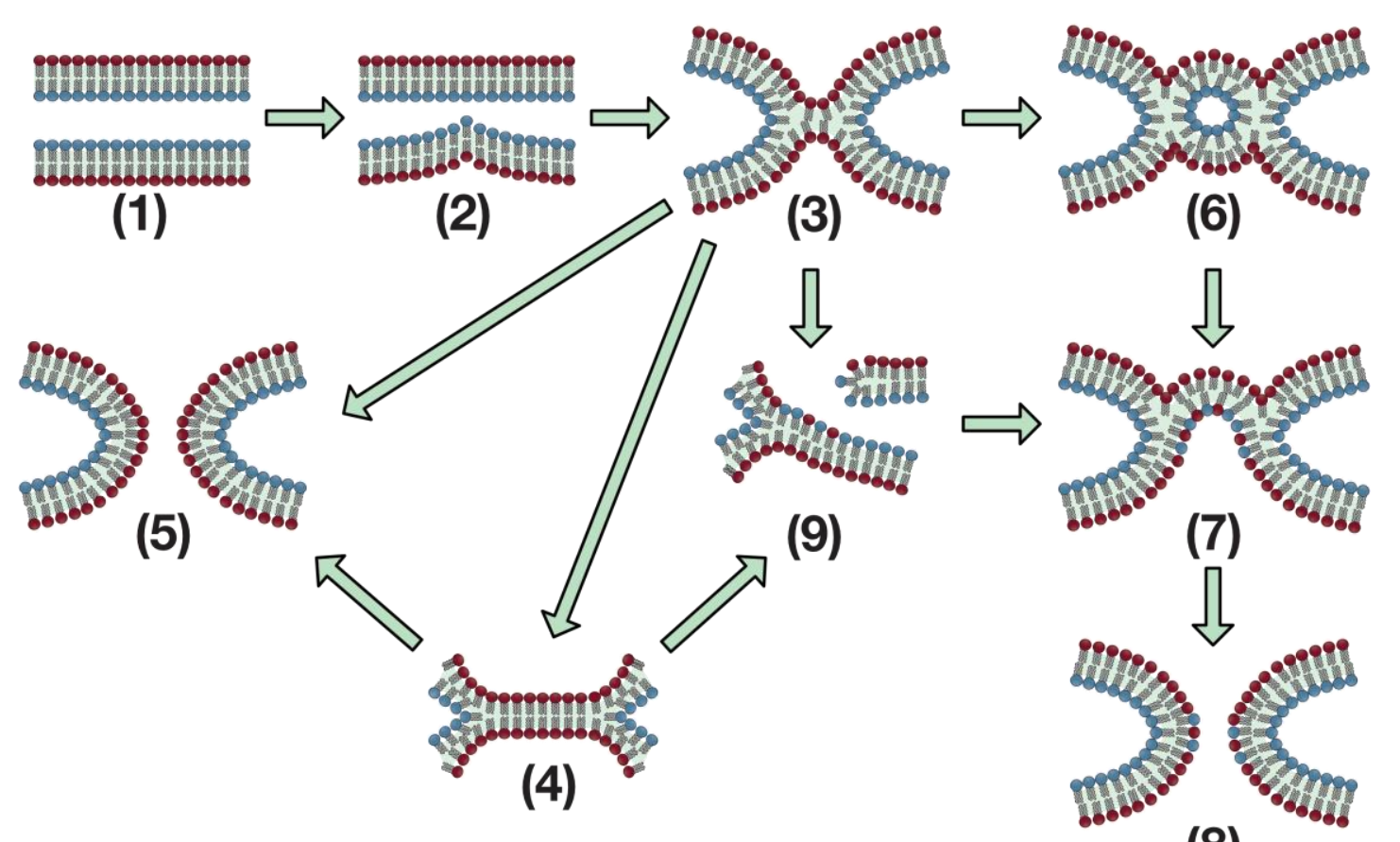

(8)

Figure 2.3: Illustration of several fusion pathways of two lipid bilayers. (1) Separate bilayers. (2) Point-like protrusion. (3) Hemifusion stalk. (4) Hemifusion diaphragm. (5) Fusion pore. (6) Inverted micelle. (7) $\pi$-shaped hemifusion diaphragm. (8) Fusion pore with mixed lipids. (9) Stalk-pore complex. Image based on ${ }^{[83,85,88,89]}$.

The second type of membrane fusion includes proteins. Here, many of the previously stated concepts are applied for the membrane merger itself. The advantages which result from the inclusion of proteins in the fusion process are manifold. The hydration, curvature, lipid 
composition or stability of the membrane can be altered due to proteins. ${ }^{[90]}$ Furthermore, proteins can actively pull membranes together to perform the fusion process. ${ }^{[7]}$ The mechanism can therefore be controlled more precisely. Some proteins capable of this task are discussed in more detail in the chapters 2.5 and 2.6.

One important factor for bilayer fusion is the lipid composition of the membrane. During the process the shapes of the bilayers change dramatically. This change has to be accommodated by the structures of the involved lipids. During stalk formation, a highly negatively curved membrane develops in the inner leaflets which has to be stabilized by a suitable lipid. As described in section 2.2, lipids with PE headgroups promote this formation, whereas lysophosphatidylcholine (LPC) inhibit the process. ${ }^{[91]}$ Conversely, LPC is better suited for the outer leaflet than PE lipids. These findings can also be applied to the curvature of liposomes. Less curved membranes tend to fuse less readily than highly curved membranes. ${ }^{[91]}$ Thus, larger liposomes are less fusogenic, whereas small liposomes, for example small unilamellar vesicles (SUVs) are more prone to fuse.

\subsection{Neuronal Exocytosis}

Since the 1960s, when the fundamental understanding about synaptic signal transduction was obtained, a lot of research was conducted in this field. Especially the exocytosis of synaptic vesicles has been studied intensively, making it one of the best understood membrane fusion processes. ${ }^{[2,92-94]}$

Transferring information between cells is a fundamental process in many organisms. In mammals, the central nervous system is filled with specialized cells to conduct reliable and fast information transduction. These so-called neurons consist of different components (see Figure 2.4a). The cell body is made of the soma which contains the nucleus and is connected to multiple branched dendrites. These dendrites receive information from other cells that is processed in the cell body. The information is then transferred via the axon, an elongation of the cell, in which electrical pulses can be transmitted over a long distance to the axon termini. The speed of the information transfer is further increased by Schwann cells, which wrap around the axon to form the myelin sheath. At the terminal points a connection to other cells e.g. other neurons form a synapse (see Figure 2.4b). At the synapse the electrical information is translated into a chemical signal that is transmitted to the next cell. 
(a)

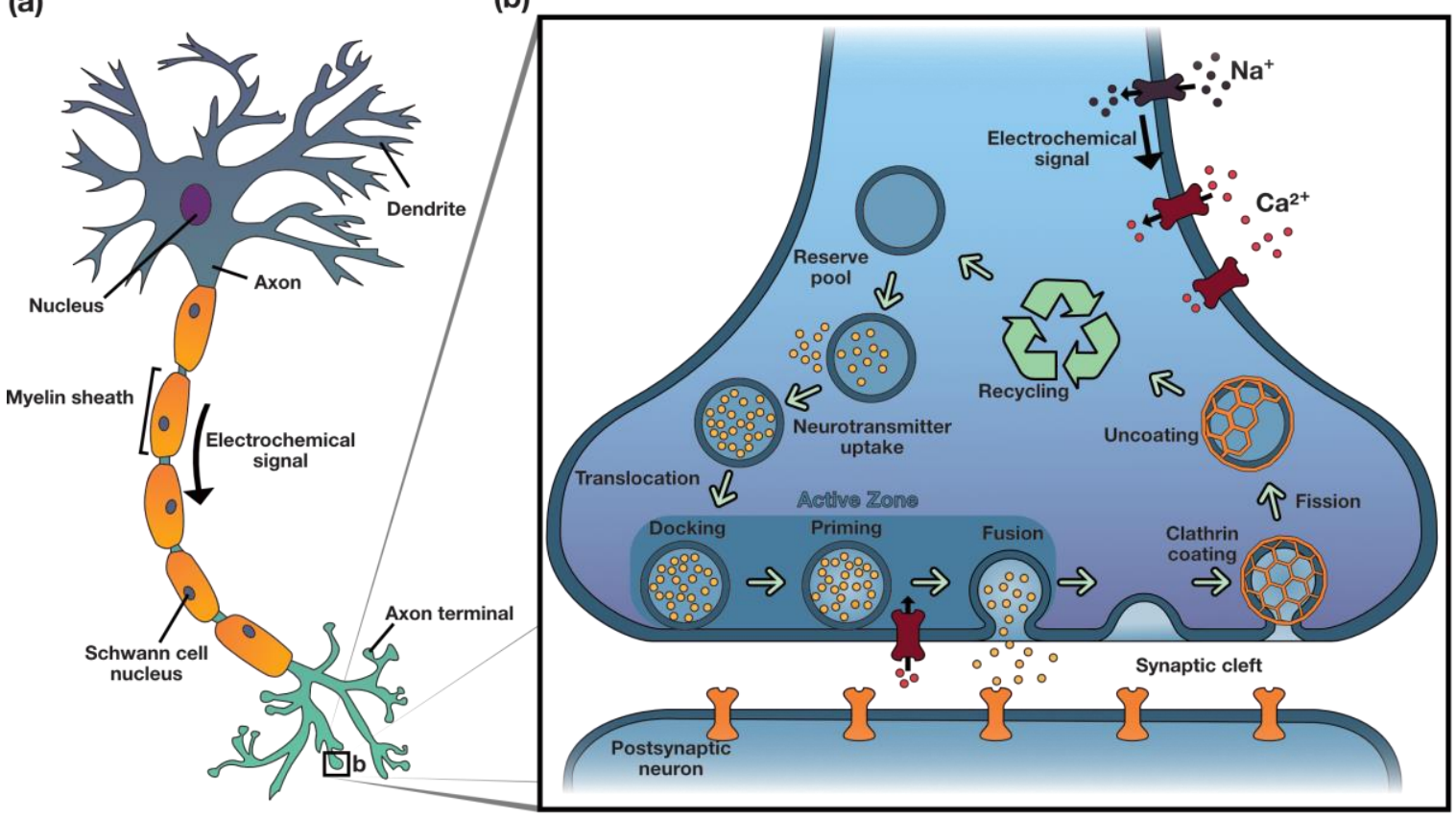

Figure 2.4: Illustration of neuronal signal transduction synaptic activity. (a) Structure of a neuron. (b) Synapse with simplified vesicle trafficking cycle. The electrical signal from the axon is translated into chemical information. Neurotransmitters are released into the synaptic cleft, where they bind onto receptors on the postsynaptic membrane. Based on ${ }^{[94]}$.

The underlying principle of the information transfer is a difference in the electrochemical potential between the intra- and extracellular area. The composition of ions differs between the inside of the neuron and the outside. Consequently, a charge difference across the membrane exists. This potential, which is maintained by active ion pumps, can be changed in the event of an action potential. An action potential is generated when signals from the dendrites arrive in the axon hillock and a specific threshold is exceeded. After that, the signal is sent down the axon and induces a change in the resting potential. This is done via a set of voltage-gated ion channels which alter the permeability of $\mathrm{Na}^{+}$and $\mathrm{K}^{+}$-ions of the membrane. Upon arrival at the axon terminal region, voltage-gated $\mathrm{Ca}^{2+}$ channels open. The previously prevalent low amount of $\mathrm{Ca}^{2+}$-ions increases and triggers multiple events, one of which is the fusion of transmitter filled synaptic vesicles with the presynaptic membrane. These transmitters are then released into the synaptic cleft and can bind to specific receptors on the postsynaptic membrane.

The formation and recycling of synaptic vesicles is a process needed for signal transduction, to maintain a readily releasable pool of neurotransmitter filled vesicles (see Figure 2.4b). ${ }^{[2]}$ 
For the neurotransmitters to be released, they first have to be incorporated inside a vesicle via active transport. Filled vesicles form the readily releasable pool of synaptic vesicles which are stored inside the cell until needed. ${ }^{[55]}$ In the next step, the filled vesicles dock at the active zone near the synaptic membrane and undergo a priming process. After priming, the vesicles can be triggered by means of a $\mathrm{Ca}^{2+}$ to take part in the fusion process. Subsequent, the empty synaptic vesicle can undergo endocytosis to be recycled via endosomes. ${ }^{[2]}$ This whole process is accompanied by a variety of proteins e.g. clathrin, to regulate and catalyze the different steps.

\subsection{Fusion Proteins}

Fusion of biological membranes can be performed by different types of proteins. Depending on the evolutionary background, the protein structures and fusion mechanisms differ quiet substantially. For example, viral fusion proteins facilitate the merger with the proteins being present on only one of the membranes. ${ }^{[96]}$ SNARE proteins on the other hand are located in both membranes and interact with each other to facilitate fusion. ${ }^{[6]}$ Knowledge about natures fusion machinery is important considering e. g. the recent outbreak of the SARS-CoV-2 virus. The fusion proteins used by the virus are potential targest for drug agents, as they are exposed to the environment. ${ }^{[97]}$ In this chapter, the different types of fusion proteins are discussed.

\subsubsection{Viral Fusion}

Viral fusion differs significantly compared to neuronal exocytosis. The acceptor membrane does not have proteins specifically designed for the fusion of viral membrane with the host. Furthermore, viral fusion proteins do not necessarily have to be recycled after use, as one fusion event leads to the desired outcome. Consequently, viruses have developed alternative methods of fusing separate membranes. ${ }^{[96]}$ The proteins used by viruses can be divided into three main classes. ${ }^{[98,99]}$ Class I fusion proteins are trimers consisting mainly of $\alpha$-helical parts. Class II proteins have a $\beta$-sheet as the most defining feature and class III fusion proteins share the features of class I and II. ${ }^{[99]}$

One of the best researched virus fusion proteins is hemagglutinin (HA) in the influenza virus, which belongs to the class I fusion proteins. ${ }^{[100,101]}$ In the case of HA, a protein trimer 
is needed for this process. The monomers consist of two domains, the globular head region $\mathrm{HA}_{1}$, which contains the receptor-binding site and the $\mathrm{HA}_{2}$ domain, which encloses the fusion peptides. ${ }^{[101,102]}$ Both are connected via a disulfide bridge. Briefly, the transmembrane viral fusion proteins form a trimer which reside in an inactive state (see Figure 2.5 step 1). Upon a specific trigger, which can be the interaction with a receptor or $\mathrm{pH}$ change, the protein changes its conformation rather drastically. During this change, $\mathrm{HA}_{1}$ folds towards the outside (2) and the fusion peptide of $\mathrm{HA}_{2}$ is exposed (3). The previously unstructured parts form $\alpha$-helices which point toward the target membrane. The fusion peptide is inserted into the target membrane (4) and further conformational changes occur, leading to a bending of the proteins (5). Meanwhile, the two membranes are pulled toward each other, creating disturbances in the bilayers. Consequently, a hemifusion diaphragm is formed (6) and the fusion pore opens, completing the process (7). ${ }^{[98]}$

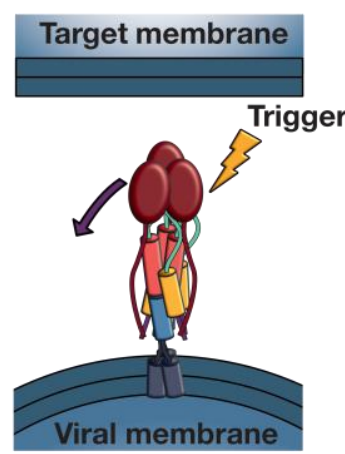

(1)

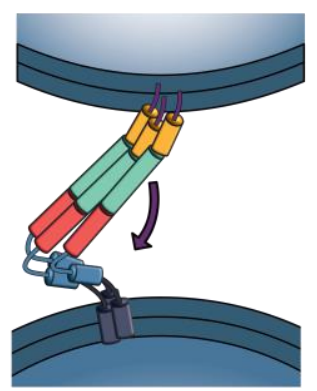

(5)

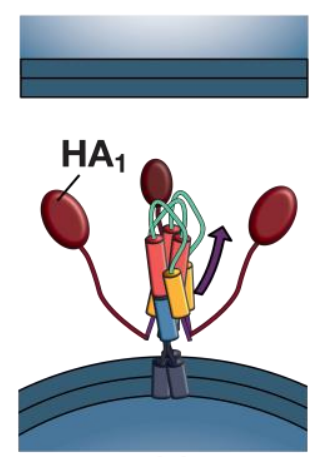

(2)

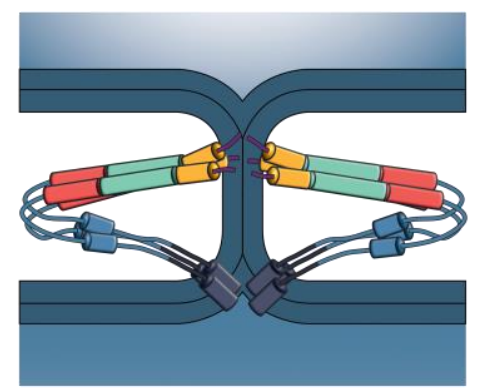

(6)

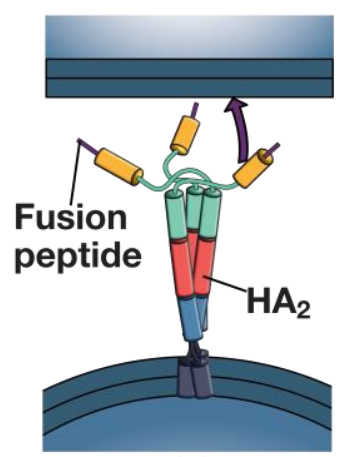

(3)

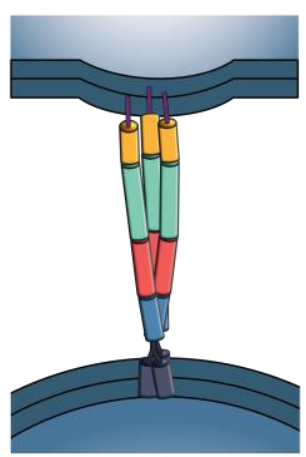

(4)

Figure 2.5: Schematic diagram of viral membrane fusion. (1) Assembled viral proteins wait for a trigger. (2) $\mathrm{HA}_{1}$ folds away from $\mathrm{HA}_{2}$ but stays connected the whole process (not shown in following steps). (3) The fusion peptide is extended. (4) Insertion of the fusion peptide into the target membrane. (5) A conformational change of the proteins pulls both membranes toward each other. (6) A fusion diaphrama develops. Multiple trimers are involved in the whole process. (7) Opening of the fusion pore. Based on ${ }^{[97,103]}$. 
One virus which gained worldwide attention in 2019/2020 is the severe acute respiratory syndrome coronavirus-2 (SARS-CoV-2). The virus causes the coronavirus disease 2019 (Covid-19) which can lead to severe respiratory malfunctions. ${ }^{[104]}$ Coronavirus (CoV) membrane fusion is achieved by the coronavirus spike protein, which assembles into trimers on the surface to form the crown-like (corona) appearance. ${ }^{[105,106]}$ It belongs to the class I fusion proteins and is therefore similar to the HA protein in structure and fusion mechanism. ${ }^{[103,107]}$ Fusion can be triggered either by the presence of the exogenous protease trypsin or the cathepsin L protease. ${ }^{[108,109]}$

\subsubsection{Mitochondrial Fusion}

In contrast to viral fusion, the mechanism for mitochondrial fusion remains elusive to this day. Key proteins involved have been identified but the mechanism is still being researched. ${ }^{[110]}$ The outstanding characteristic of mitochondrial fusion is the even more complex task of fusing four membranes, as mitochondria consist of an outer- and inner mitochondrial membrane, which use a different set of fusion proteins. ${ }^{[111]}$

The proteins involved in mitochondrial membrane fusion are part of the dynamin-related proteins. ${ }^{[110,112]}$ These proteins are a group of GTPases which main task is to shape membranes. The process starts with two MFN1 (mitofusin 1) molecules on opposite mitochondria docking to each other. The association triggers conformational changes which lead to GTP hydrolysis and induces the fusion of the outer mitochondrial membrane. ${ }^{[113]}$ The next step is the fusion of the mitochondrial inner membranes, which is achieved by OPa1 (optic atrophy protein 1) and MFN2. Fusion and fission of the mitochondrial membrane appears to be an integral part of many essential cell processes like cell signaling, apoptosis or mitophagy. ${ }^{[12]}$

\subsubsection{Cell-cell Fusion}

Fusion of multiple cells is an essential mechanism in mammals. Nevertheless, only a limited collection of cells has this ability. For example, fertilization is needed for proliferation of the species and includes a cell-cell fusion event. Here, the sperm fuses with the oocyte to convey information in form of its DNA. Immune responses also include such events and are crucial to maintain health of its host. ${ }^{[98]}$ Macrophages are mononucleate cells whose range of tasks is quiet big owing to their high mobility, plasticity and 
adaptability. ${ }^{[114]}$ In certain conditions, fusion between two macrophages occurs to generate a multinucleated osteoclast, which has multiple tasks in bones. ${ }^{[115]}$ Skeletal muscles are also a product of cell-cell fusion, in fact, fusion of multiple myoblasts is needed to form a single muscle fiber. ${ }^{[116]}$

Further proteins capable of fusing membranes, have been summarized as FF-Proteins (fusion family). ${ }^{[117]}$ These proteins are a superfamily referred to as fusexins and are required in both fusing membranes. Upon docking to one another, trimers form during the fusion process, where one of the proteins is on the opposing membranes compared to the other two. After a relocation process, the two membranes get pulled toward each other and a supposedly zippering-like process executes the fusion. ${ }^{[118]}$ The most prominent proteins of this family are EFF-1(epithelial fusion failure 1) and AFF-1 (anchor-cell fusion failure 1), which are related to membrane glycoproteins found in C. elegans. ${ }^{[119,120]}$

\subsection{SNARE Proteins}

Beside the previously mentioned membrane fusion proteins, a different family of proteins is responsible for membrane fusion in the secretory pathway. SNAREs (soluble $\mathrm{N}$-ethylmaleimide-sensitive factor attachment protein receptor) have been studied since the late 1980s and identified as a key complex in membrane fusion. ${ }^{[6]}$ Though, a lot of research has been conducted already, SNAREs remain a point of interest concerning their fusion behavior to this day. In this chapter, the fusion mechanism and structure of the SNARE machinery is discussed. Furthermore, important associated and regulative proteins which are also involved in SNARE mediated membrane fusion are examined.

\subsubsection{Structure of SNARE Proteins}

For neuronal exocytosis the SNARE machinery consists of synaptobrevin 2, syntaxin $1 \mathrm{~A}$ and SNAP-25 (25 kDa synaptosome-associated protein). Syntaxin 1A and SNAP-25 are located at the presynaptic membrane, whereas synaptobrevin 2 can be found on the synaptic vesicle. On the basis of the protein location, syntaxin 1A and SNAP-25 were historically classified as t-SNAREs (target-membrane) and synaptobrevin 2 as a v-SNARE (vesicle membrane). Attachment to the membrane is achieved either through a peptidic TMD (trans 
membrane domain) or a lipid anchor (see Figure 2.6). For example, SNAP-25 uses palmitoyl chains to attach to the membrane whereas synaptobrevin 2 and syntaxin $1 \mathrm{~A}$ have a TMD at the C-terminus. ${ }^{[6]}$

(a)

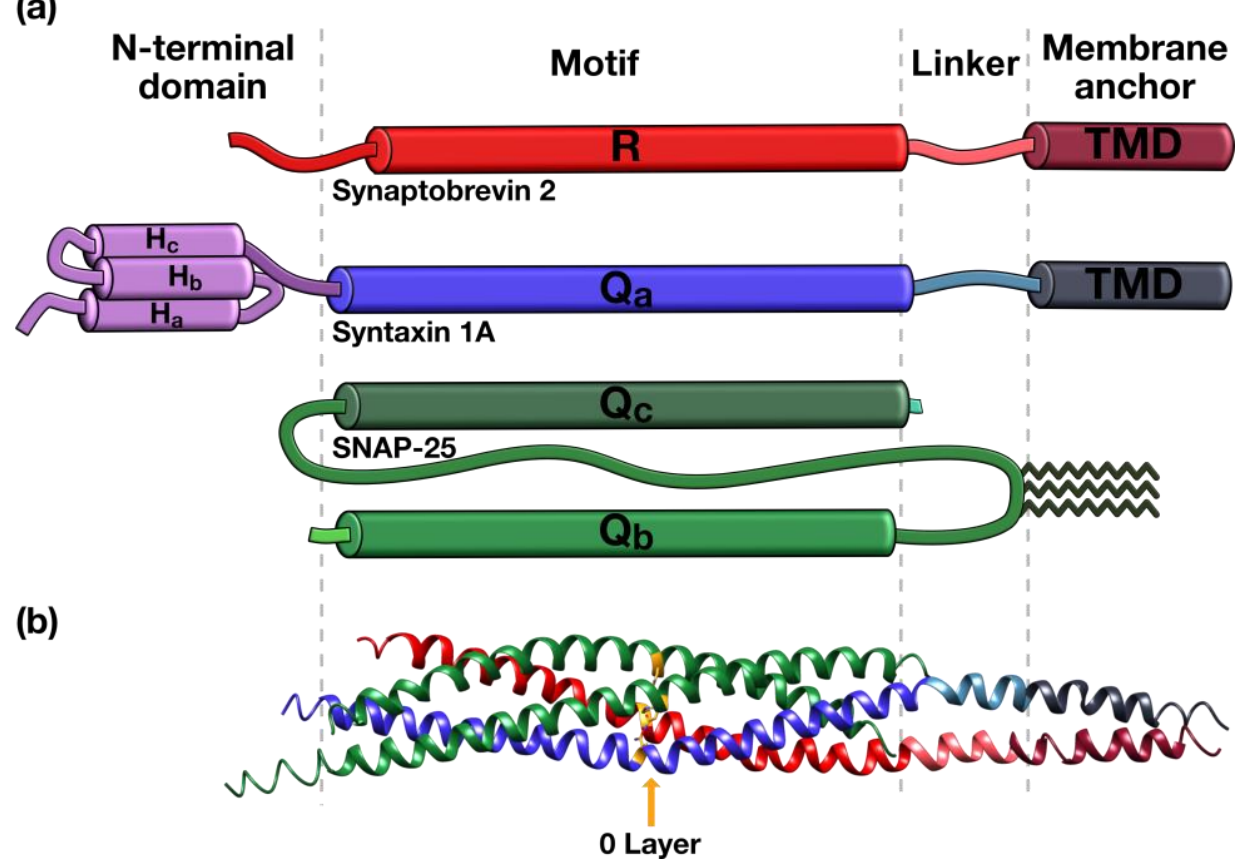

Figure 2.6: Illustration of the structure of three neuronal SNARE proteins. (a) Synaptobrevin 2 and syntaxin 1A have peptidic membrane anchors, whereas SNAP-25 has lipids attached to its peptidic backbone. The subunits $\mathrm{Q}_{b}$ and $\mathrm{Q}_{\mathrm{c}}$ are connected via a peptide chain. The chain is not part of the motif. (b) X-ray structure of the assembled SNARE complex with marked 0-layer. X-ray data from STEIN et al. ${ }^{[23]}$ Molecular graphics and analyses were performed with the UCSF Chimera package. ${ }^{[121]}$

The structure of SNAREs can be divided into domains which serve different functions. The most characteristic domain is the SNARE motif which consists of a 60-70 amino acid long evolutionary conserved sequence. Four of those motifs form a tetrameric coiled coil, yielding the core SNARE complex. ${ }^{[6]}$ In case of the neuronal SNAREs, syntaxin and synaptobrevin contribute one $\alpha$-helix each, whereas SNAP-25 contributes two motifs, which are connected to each other via a peptidic loop. The core complex consists of 16 layers of mostly hydrophobic amino acids, which interact with each other to form a stable parallel coiled coil. In the center of this bundle, three arginine $(\mathrm{R})$ and one glutamine $(\mathrm{Q})$ residue form the so called "zero layer". ${ }^{[3]}$ These specific residues are highly conserved in the SNARE family. Due to this, SNARE proteins are classified as R- or Q-SNAREs (see Figure 2.6). ${ }^{[122]}$ The SNARE complex is a remarkably strong structure, which needs 
multiple ATP molecules for disassembly. ${ }^{[123,124]}$ The energy which is released during the formation is used to bring the opposing membranes into close proximity. ${ }^{[77,125,126]}$

On the C-terminal end of the motif, a short linker connects it to the TMD. The linker in syntaxin $1 \mathrm{~A}$ and synaptobrevin 2 are a sequence of ten amino acids each which reside in the junction between lipid membrane and cytosol. ${ }^{[6,23]}$ The structure of the linker, especially its stiffness or flexibility is being discussed in literature extensively as described in section 2.6.4. ${ }^{[127-130]}$ The TMD is located on the C-terminal end of the proteins and consists of about 20 amino acids with mainly hydrophobic side chains. ${ }^{[10,23]}$ These form primarily an $\alpha$-helical structure in the membrane. Recent molecular dynamics (MD) simulations suggest, that the TMD specifically of synaptobrevin has a flexible glycine kink, which modulates the insertion angle. ${ }^{[129]}$ Furthermore, the linker and N-terminal half of synaptobrevins TMD (residues 85-99) form a continuous helix which is decoupled from the C-terminal part of the TMD (101-116) due to the glycine in position $100{ }^{[130]}$ Also, the simulations suggest a tilt between $30^{\circ}$ to $40^{\circ}$ in a POPC membrane for the TMD of synaptobrevin. ${ }^{[129,130]}$

At the N-terminal end of the proteins, additional domains can be located which serve many different functions. ${ }^{[6,131]}$ For example, the N-terminal domain in syntaxin $1 \mathrm{~A}$ forms an antiparallel trimeric helix bundle which interacts with the motif, resulting in two conformations. ${ }^{[132]}$ In the closed conformation, the $\mathrm{H}_{\mathrm{abc}}$ domain is folded onto the $\mathrm{N}$-terminal part of the motif. In this conformation, the regulatory protein munc18-1 (mammalian uncoordinated-18) can bind to syntaxin 1A, inhibiting the formation of the SNARE core complex. Upon release of munc18-1, via the help of additional proteins, syntaxin converts to the open state, in which the core SNARE-complex formation takes place. ${ }^{[133]}$ The question whether the domain is essential for the fusion process can not be generally answered. For some SNAREs (Sso1p of yeast) the domain is necessary, ${ }^{[134]}$ whereas for others (Vamp3p of yeast) the domain is dispensable. ${ }^{[135]}$ Additionally the $\mathrm{N}$-terminal domain can serve as a docking point for different other regulatory proteins. ${ }^{[136]}$

\subsubsection{Formation of the SNARE Complex}

The assembly of the core SNARE complex and the succeeding membrane fusion have yet to be understood in its entirety. Due to the many proteins associated to this process, especially for regulation, a generally accepted mechanism remains to be found. 
Nevertheless, the "zippering" hypothesis of SNARE fusion is the most recognized and describes the fusion process to start at the $\mathrm{N}$-termini and proceeding toward the $\mathrm{C}$-termini in a zipper-like manner (see Figure 2.7). ${ }^{[7]}$

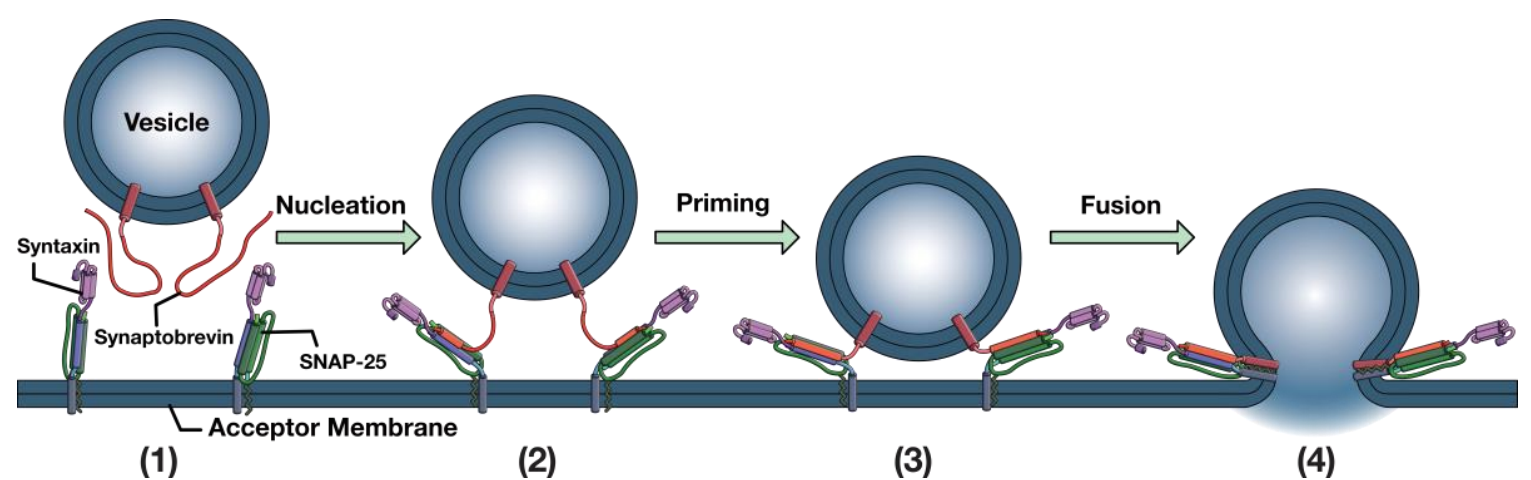

Figure 2.7: Illustration of the SNARE zippering mechanism. (1) Syntaxin 1A and SNAP-25 pre-assemble on the acceptor membrane. The vesicle with synaptobrevin 2 comes into proximity of the other proteins. (2) Formation of the half-zippered state at the N-termini of the motif. (3) Zippering continues toward the C-termini. (4) Completed zippering leads to pore opening. The SNARE proteins are now all in the same membrane and form the cis-SNARE complex. Based on ${ }^{[6]}$.

Recent studies suggest the zippering to proceed in distinct steps. ${ }^{[9,137,138]}$ It starts with the association of the N-terminal domain of the motif, which leads to the formation of a halfzippered state. This process is slow and believed to be the rate determining step of the whole assembly. ${ }^{[139]}$ However, during this stage the readily releasable vesicle pool is build which ensures a fast signal transduction between nerve cells. The half-zippered state was detected by several research groups with a variety of methods. Using single molecule FRET and EPR (electron paramagnetic resonance), the SNAREpin assembly was shown to form a helical structure right above the conserved ionic layer, whereas the $\mathrm{C}$-terminal structure was determined ambiguous. ${ }^{[140]}$ Another study using magnetic tweezers found similar results and also verified the directionality of the complex formation to go from $\mathrm{N}$ - to C-termini. ${ }^{[141]}$ The intermediate state is believed to be important for the organization of the regulatory machinery. ${ }^{[139,142,143]}$ The next step includes the zippering of the C-terminal domain which is accompanied by a high energy release. ${ }^{[9]}$ Furthermore, it is believed that zippering continues throughout the linker and the TMD to trigger fusion of the membranes. ${ }^{[144-146]}$ At this stage, all SNARE proteins are in the same membrane forming the cis-SNARE complex. Although, the different energy stages of the assembly were shown with multiple methods, it remains unclear if the natural process halts at these positions. ${ }^{[147]}$ 


\subsubsection{Regulation of the SNARE Complex Formation}

Albeit the SNARE proteins resemble the minimal machinery needed to facilitate the fusion of the synaptic vesicles and the presynaptic membrane, many more proteins are involved in this process. The roles of the regulatory proteins include the priming of the complex, activation of proteins, sensing of triggers and many more. ${ }^{[6,148-150]}$ Although, over three decades of research have been done in this field, the exact role of each protein has yet to be determined. ${ }^{[148,151,152]}$

Munc18-1 was one of the first proteins found to interact with syntaxin 1A. It is arch-shaped, consists of three domains and belongs to the SM (Sec1/Munc18-like) protein family. ${ }^{[153]} \mathrm{A}$ large central cavity provides the binding surface for the closed state of syntaxin $1 \mathrm{~A}$. The $\mathrm{N}$-terminal region of syntaxin including the $\mathrm{N}$-peptide and $\mathrm{H}_{\mathrm{abc}}$ domains as well as the fourhelix bundle are points of contact with munc18-1. ${ }^{[153]}$ The exact role of the SM protein is still under debate. ${ }^{[154]}$ One task involves the stabilization of the SNARE complex in a primed state. ${ }^{[155]}$ However, recent research supports the idea of munc18-1 remaining associated with the SNARE complex during the fusion process, making it an essential component of the fusion mechanism. ${ }^{[150,156]}$ Accordingly, a complex between syntaxin $1 \mathrm{~A}$ and munc18-1 rather than with SNAP-25 is currently debated to be the beginning of the fusion process (see Figure 2.8). ${ }^{[157]}$

Another protein associated to the SNARE complex assembly is munc13. ${ }^{[152]}$ This protein has a large ( $200 \mathrm{kDa})$ arche-shaped multidomain structure and is involved in the opening of the conformation of syntaxin $1 \mathrm{~A} \cdot{ }^{[158]}$ It is also capable of bridging both involved membranes due to its interaction with DAG and $\mathrm{PIP}_{2}$. Furthermore, munc13 assists in the formation of the ternary SNARE complex between syntaxin, synaptobrevin and SNAP-25. ${ }^{[159]}$ The cooperation of munc18-1 and munc13 ensure the formation of the fourhelix bundle and keep the system in a primed state. 


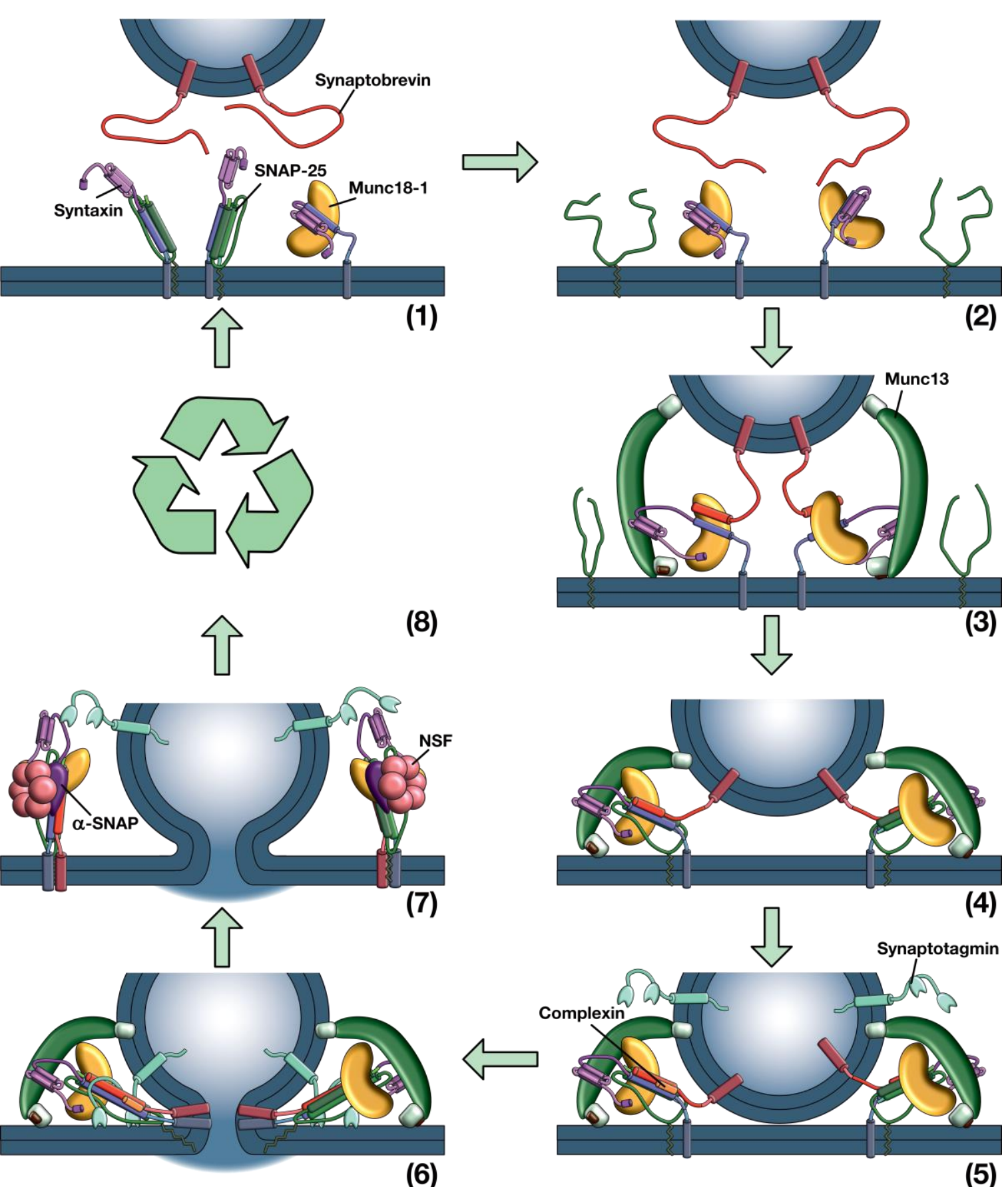

(6)

Figure 2.8: Schematic illustration of the recent presumed fusion cycle of synaptic vesicles. (1) Syntaxin 1A and SNAP-25 are forming a complex which is presumably disassembled by NSF and SNAPs. (2) After disassembly, munc18-1 and syntaxin $1 \mathrm{~A}$ in its closed state form the starting complex. (3) Munc13 connects to both membranes and interacts with the N-terminal domains of syntaxin 1A. Synaptobrevin attaches to the munc18-1/syntaxin 1A complex. (4) Munc18-1 acts as a template to initiate the motif assembly including SNAP-25. (5) Complexin attaches to the four-helical bundle. (6) A calcium trigger activates synaptotagmin, which attaches to the motif ensamble to initiate membrane fusion. (7) NSF and SNAPs disassemble the fusion machinery post fusion. (8) The proteins are recycled and used in consecutive fusions. Mechanism based on ${ }^{[1,152]}$. 
In neuronal exocytosis $\mathrm{Ca}^{2+}$ influx triggers the release of the neurotransmitters into the synaptic cleft. Sensing the change in the concentration is associated with synaptotagmins. ${ }^{[160]}$ Synaptotagmin 1 (Syt1) can be classified as an evolutionary conserved transmembrane protein which is located in the synaptic vesicle. Beside the transmembrane domain it has two connected domains $\mathrm{C} 2 \mathrm{~A}$ and $\mathrm{C} 2 \mathrm{~B}$ which are capable of binding multiple $\mathrm{Ca}^{2+}$-ions. ${ }^{[161]}$ After calcium binding, synaptotagmin stimulates membrane fusion by interacting with the target membrane. ${ }^{[162]}$ However, the proteins task does involve many more functions. For example, another task which is associated to synaptotagmin is its ability to act as a fusion clamp for the SNARE complex. ${ }^{[160]}$ This topic has been discussed controversially over the past years, as other proteins were suspected to perform in the same way. ${ }^{[163,164]}$ Nevertheless, recent research appoints this function to the C2B domain of synaptotagmin. ${ }^{[165]}$

Another protein group involved in the SNARE mediated fusion mechanism are complexins (Cpx). Complexins and synaptotagmins have a concurrent binding behavior to the SNARE complex. The interplay of the SNARE-Cpx-Syt1 tripartite is specific, as X-ray data of the complex showed a high complementarity between the molecules. ${ }^{[166]}$ Collaboration of these proteins leads to a successful $\mathrm{Ca}^{2+}$-triggered neurotransmitter release.

After the membrane fusion process is completed, the SNARE complex is disassembled and recycled for further uses. The dismantling of the protein complex is an integral part of the whole process as many consecutive fusions have to be performed in a short time. The whole procedure is organized by NSF (N-ethylmaleimide-sensitive factor) and SNAPs (soluble NSF attachment proteins). NSF is an ATPase that binds together with SNAPs to the assembled SNARE proteins to form a supercomplex. ${ }^{[167]}$ ATP hydrolysis provides the necessary energy to execute the disassembly. However, the exact mechanism remains elusive and is topic of research interest until today. ${ }^{[124]}$

\subsubsection{Role of the Linker in SNARE mediated Fusion}

The linker connects the characteristic SNARE motif with the transmembrane domain. The exact role of the linker during the fusion process is under investigation for years. Most research focused on the flexibility of this domain and its ability to direct the energy generated by the motif assembly towards the TMD. ${ }^{[6,168]}$ Experiments as well as MD simulations indicate an ambivalent characteristic regarding the stiffness, which is necessary for efficient fusion. ${ }^{[168]}$ Stiffness is achieved by the formation of an $\alpha$-helix, which 
compared to a random coil is limited in its flexibility. A stiff linker is needed to ensure a force propagation from the motifs to the TMDs. ${ }^{[127]}$ Mechanical stress stored in the linker leads to a self-organization which conducts the membrane fusion process. Stress cannot be propagated beyond the linker if it is too flexible, MD simulations have shown. ${ }^{[127]}$ Furthermore, the X-ray structure of the post-fusion cis-SNARE complex shows a fully helical TMD of synaptobrevin and syntaxin, suggesting it to be the end state. ${ }^{[23]}$ The X-ray structure of the natural SNARE complex of syntaxin 1A and synaptobrevin 2 shows multiple aromatic residues present in the linker (see Figure 2.9). These are arranged in such a way, that they wrap around the synaptobrevin linker to form a collar of aromatic residues. It is assumed that these aromatic residues stiffen the linker region due to intermolecular interactions. ${ }^{[23]}$ Furthermore, they appear to modulate insertion depth of the TMD into the membrane. ${ }^{[169]}$

(a)

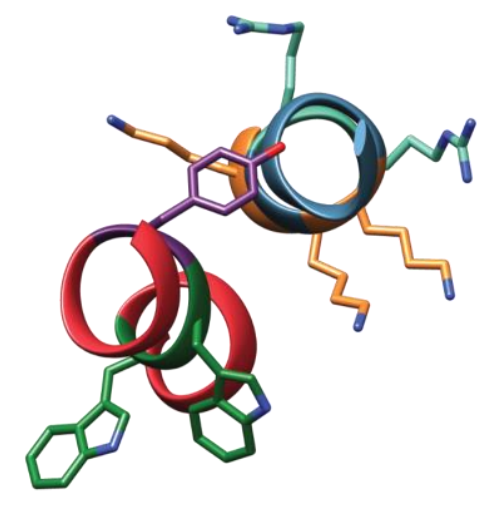

(c) (b)

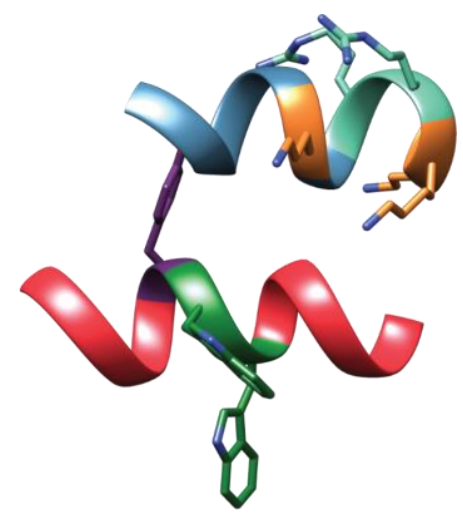

Syntaxin 1A KYQS KARRKK

Synaptobrevin 2 KRKYWWKNLK

Figure 2.9: Illustration of the linkers of synaptobrevin 2 and syntaxin 1A. (a) Top view from the N-termini; (b) side view with $\mathrm{N}$-termini on the left; (c) amino acid sequence of the linker domains of both proteins. X-ray data from STEIN et al. ${ }^{[23]}$ Molecular graphics and analyses were performed with the UCSF Chimera package. ${ }^{[121]}$

As the linkers of syntaxin and synaptobrevin are small, research focused on the specific amino acids present in the linkers. For this, different mutations, ranging from amino acid substitutions to the insertion of small peptides into the linker have been done. Previous deletion experiments of specific amino acids in synaptobrevins linker showed, that fusion can still be observed albeit with a loss in the efficiency. ${ }^{[77]}$ Regarding the linker length, opposing results were reported with different experimental setups. Thereafter, linker 
elongation may prolong the time between signal stimulus and exocytosis. ${ }^{[170]}$ Furthermore, small changes by adding six additional amino acids does not have significant effects in in vivo experiments. Whereas, the addition of eleven or more amino acids to the linker have an impact on the growing behavior of Saccharomyces cerevisiae. ${ }^{[25]}$ Unfortunately, there is still a lot of controversy regarding the linker. Disrupting the helicity of the linker yielded mixed results in literature. While overexpression of a mutant with two proline residues shows growth, using endogenous levels of the target protein did not. ${ }^{[25]}$ This phenomenon was also observed in hippocampal neurons by addition of twelve and 24 amino acids. ${ }^{[171]}$ The polybasic residues are a highly conserved motif in syntaxin $1 \mathrm{~A} .{ }^{[172]}$ Multiple functions and mechanisms have been reported to be linked to this region of the protein, making it essential for the fusion process. Especially the high density of positive charges and the position in the membrane is important regarding the surrounding lipids. ${ }^{[2]}$ Studies conducted with Sso1p showed that substitution of the polybasic area in the juxtamembrane region leads to a total loss of function of the protein. ${ }^{[25]}$ Depending on the experiment, a substitution of as few as three residue changes showed a complete stop in activity. However, the exact reason for this remains unclear, as the results could not be reproduced with artificial liposomes. ${ }^{[25]}$ Furthermore, the polybasic area is known to interact with head groups of lipids due to its charge and position in the membrane. ${ }^{[172]}$ The recruitment of these lipids may therefore also be a feature of these amino acids.

\subsection{Model Systems for SNARE Fusion}

Studying the SNARE machinery can be done with an assortment of approaches. The direct way is to examine the proteins by themselves. Another possibility is to use molecules which resemble the natural systems function or structural features. Over the years, many different model systems have been developed to mimic the membrane fusion capabilities of SNAREs. Due to the high complexity of the natural proteins and whole exocytotic pathway, a need for more simple assemblies arose to isolate specific parts of the process. The focus of these structures varies from being easy to synthesize to maintaining key parts of the natural sequences. In the following chapter, some of these SNARE analogs are presented and discussed regarding their specific features. 


\subsubsection{Simplified SNARE Complexes}

One way of studying the SNARE machinery is to isolate the proteins and use them in in vitro experiments. The advantage of this method is that other proteins and molecules are excluded from the experiment while studying the targeted component. The observed results can be assigned beyond doubt to the isolated proteins. With this approach, the SNAREs were identified to be the minimal machinery needed to perform membrane fusion. ${ }^{[173]}$ However, extraction of the proteins from their natural surroundings changes several important factors. Due to this, contradicting results were obtained in many studies comparing in vitro with in vivo experiments. ${ }^{[174]}$ Especially, the time needed to facilitate fusion differed drastically. From the below millisecond fusion times in neurons, ${ }^{[175]}$ to several hours until completion arose questions whether this approach is reasonable. These concerns were addressed by the introduction of the $\Delta \mathrm{N}$-complex, a stabilized syntaxin/SNAP25 acceptor assembly which is capable of doing the task of membrane fusion in reasonable times (Figure 2.10). ${ }^{[7]} \mathrm{A}$ short fragment of synaptobrevins C-terminal region (AA 49-96) prevents the formation of the inactive 2:1 complex which consists of two syntaxin and one SNAP-25 protein. During the fusion process, the small fragment is replaced by the full-length synaptobrevin 2 . In comparison to the $1: 1$ complex, the $\Delta \mathrm{N}$-complex shows a highly increased liposome fusion rate.

(a)

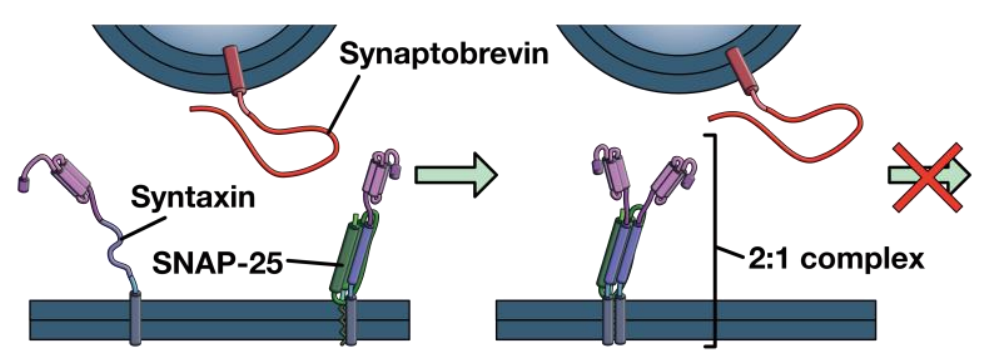

(b)

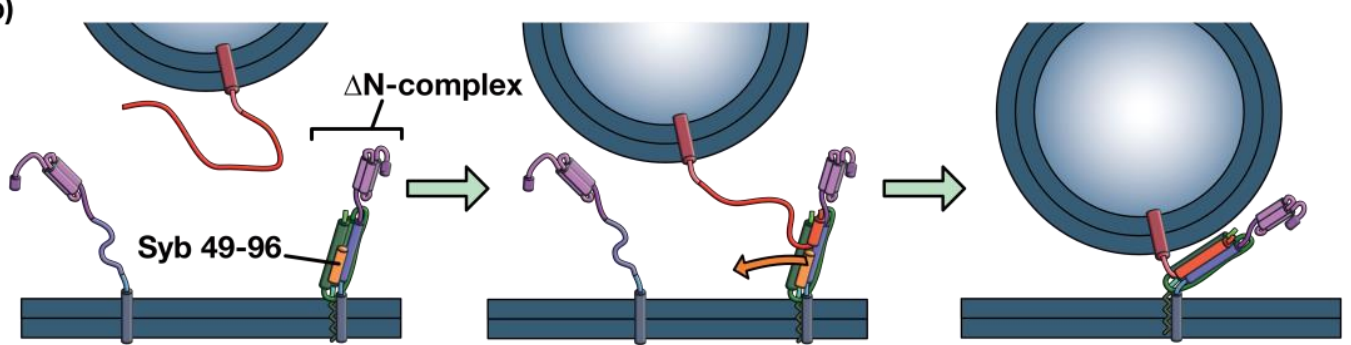

Figure 2.10: Illustration of the assembly pathway of SNAREs. (a) Formation of the 2:1 complex which reduces the fusion activity in in vitro experiments. (b) Demonstration of the Syb 49-69 fragment stabilizing syntaxin and SNAP-25. The $\Delta \mathrm{N}$-complex prevents the 2:1 complex formation and improves the fusion speed of the system. Based on ${ }^{[7]}$. 


\subsubsection{Artificial SNARE Analogs}

Although, isolation of the SNARE proteins made research more accessible and easier to understand, an alternative approach reduces the complexity even further. Instead of using the natural system as a whole, only specific parts are used and combined with artificial components. It is also possible to substitute every aspect with a mimetic, to study the general mechanism or structure. The key features of the distinct SNARE domains are imitated by simpler structures. For example, instead of a four-helical-bundle as the natural recognition center, an interaction between only two artificial motifs is used. The approach of breaking down the SNAREs key features and substituting them with artificial components has previously shown to be successful in mimicking the fusion process.

The recognition unit can consist of small molecules like melamine and cyanuric acid. ${ }^{[176]}$ These are attached to a lipid anchor which are embedded in opposing liposomes. Hydrogen bonds between melamine and cyanuric acid are formed, which is enough to facilitate fusion between the two liposomes. Similar results could be obtained with the use of boronic acid and diols as the recognition unit. ${ }^{[18]}$ In this system, an additional PEG linker was attached between the boronic head group and lipid anchor to prevent the recognition unit from being buried in the hydration layer. Another example for a fairly simple fusion system was developed recently and utilizes a strain-promoted alkyne-azide cycloaddition. ${ }^{[177]}$ A click reaction between the alkine and azide fusogenes, which reside in opposing liposomes, induces membrane fusion.

A more sophisticated approach of mimicking SNARE recognition utilizes bigger molecules which can be programmed to have a high specificity. It has been shown that DNA mediated liposome fusion can be achieved by the attachment of two complementary DNA strands in the right orientation to a phospholipid anchor. ${ }^{[178]}$ One strand is attached at the 5 '-terminus and the complementary strand in the opposing liposome is connected at the 3'-terminus to the anchor. A similar approach based on DNA as the motif uses a double cholesterol anchor (Figure 2.11). ${ }^{[20,179]}$ The strands in the vesicles are already in a duplex formation, with one strand being longer and partly not participating in nucleobase pairing. This end is capable of interacting with the complementary strand in the other liposome and facilitates fusion in a zipper-like manner. The shorter ends also start interacting, leading to the generation of a short helix. 


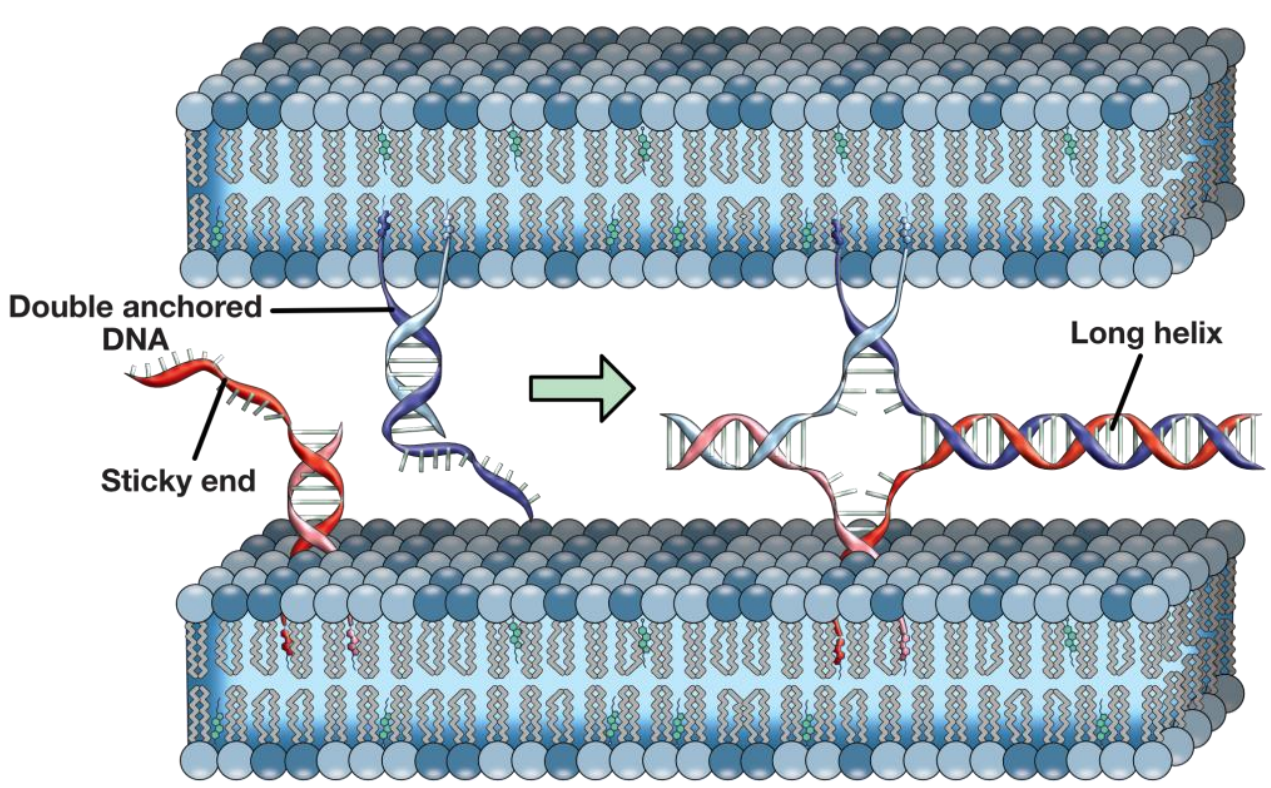

Figure 2.11: Illustration of the DNA based SNARE analog from STENGEL et al. ${ }^{[20]}$ Both liposomes contain different sets of DNA double helices which are attached to the membrane by two cholesterol anchors. One of the two DNA strands is longer and acts as a sticky end to catch the complementary strand of the opposite liposome. The formation of a short and long helix pulls both membranes together, which facilitates fusion.

A DNA alternative has been found to be a good motif for fusogens as well. Peptide nucleic acids (PNA) use a peptidic backbone onto which the nucleobases are attached. ${ }^{[180,181]}$ The recognition between complementary strands follows the Watson-Crick pairing and can be easily modified. Stability and strand orientation can be modulated by exchanging nucleobases in the sequence. Recently, PNA was attached to a PEG linker and lipid anchor to successfully promote membrane fusion. ${ }^{[182]}$

It is also possible to combine both natural and artificial structures into one model system. Thereby, more insight about the specific domains of the natural proteins can be gained. An example for this approach is the PNA-TMD model system introduced by LYGINA et al (see Figure 2.12). ${ }^{[183]}$ PNA was used as the motif and was attached to the TMD of the natural proteins syntaxin $1 \mathrm{~A}$ and synaptobrevin 2 . With this system, the effect of the orientation of duplex assembly on fusion efficiency was tested. The antiparallel orientation showed a lower amount of lipid mixing compared to parallel aligned species. As a result of the composition of the model system, the TMD could be investigated regarding several other aspects. First, the use of identical or truncated TMDs showed a significant reduction in fusion efficiency. Furthermore, the charges at the C-terminus have been altered by WEHLAND et al. to inquire into their role during membrane fusion. ${ }^{[184]}$ Normally the 
C-terminus is negatively charged due to its carboxylic group. However, changing it to a neutral amide terminus showed an increased amount of lipid mixing. An increase was also observed by the use of the net neutrally charged amino acid lysine at the C-terminal position. The introduction of a doubly anionic charge on the other hand reduced the systems fusion capabilities.

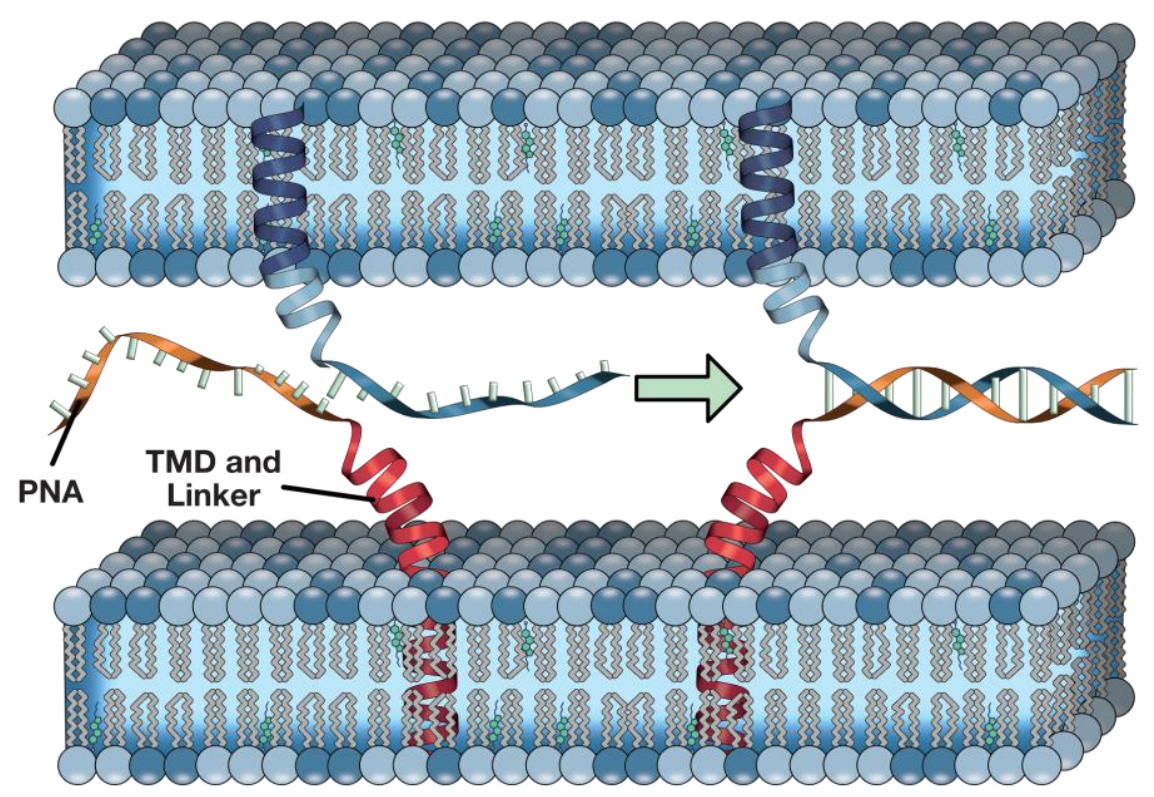

Figure 2.12: Illustration of the PNA-TMD model system by LYGINA et al. ${ }^{[183]}$ The recognition motif consisting of complementary PNA strands which are attached to the linker and TMD of synaptobrevin 2 (red) and syntaxin 1A (blue). WatsonCrick base pairing between the PNA motifs in the opposing membranes ultimately leads to fusion of the lipid bilayers.

The PNA-TMD model system was developed even further to incorporate two types of PNA. $N$-(2-aminoethyl)glycine (aeg-PNA) and alanyl-PNA which differ in their duplex formation kinetics and topology. ${ }^{[185]}$ A duplex consisting of aeg-PNA forms a helix, whereas alanyl-PNA strands form a linear structure. ${ }^{[186,187]}$ The idea behind the hybrid motif was to force a directional zippering of the peptides and draw comparisons to the native mechanism. However, the use of only five aeg-PNA building blocks turned out to be the most optimal combination for maximal fusion efficiency. ${ }^{[188]}$ Another version of this analog uses $\beta$-peptides and $\beta$-PNA and was used to modulate the distance between the two liposome species. ${ }^{[189]}$ Although the duplex formation was oriented in an antiparallel manner, lipid mixing has been observed. 


\subsubsection{E3/K3-PEG-Lipid Model System}

An alternative model system for SNARE mediated membrane fusion was developed by MARSDEN et al. ${ }^{[19,190,191]}$ Here, the three key components of the minimal SNARE fusion machinery; motif, linker and TMD were substituted by artificial structures. The transmembrane region was exchanged with a lipid or cholesterol anchor. A PEG linker with varying length was attached to the anchor to connect it to the artificial motif consisting of the heterospecific duplex peptides E3/K3. The E/K-coiled coil was first introduced by LITOWSKI et al. for the purpose of peptide delivery and as a capture system e.g. in purifications (Figure 2.13). ${ }^{[21]}$ Coiled coils are generally designed following a heptad repeat pattern denoted abcdefg. ${ }^{[192,193]}$ The residues at the positions $a$ and $d$ are exclusively hydrophobic residues which upon coiled coil formation pack into knobs-into-holes to form a hydrophobic core. ${ }^{[194]}$ Depending on the type of hydrophobic amino acid (leucine, isoleucine, valine, alanine), the formation of two-, three- or four-stranded coiled coils is preferred. ${ }^{[195]}$ In case of the E3/K3 coiled coil, positions $e$ and $g$ are occupied either with glutamic acids $(\mathrm{E})$ or lysines $(\mathrm{K})$ depending on the peptide species and are hampering the formation of homomeric coiled coils. The remaining positions are occupied by alanines or a contrarily charged amino acid to reduce the net charge of the peptide. These positions are not taking part in the coiled coil interaction and can therefore be modified more forgivingly. These heptads are repeated three times each to form the E3 or K3 peptide respectively.

(a)

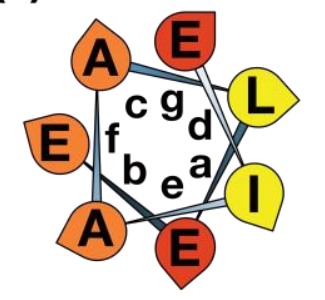

(c)

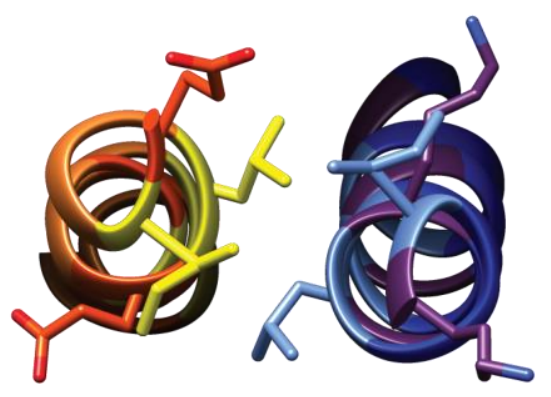

(b)
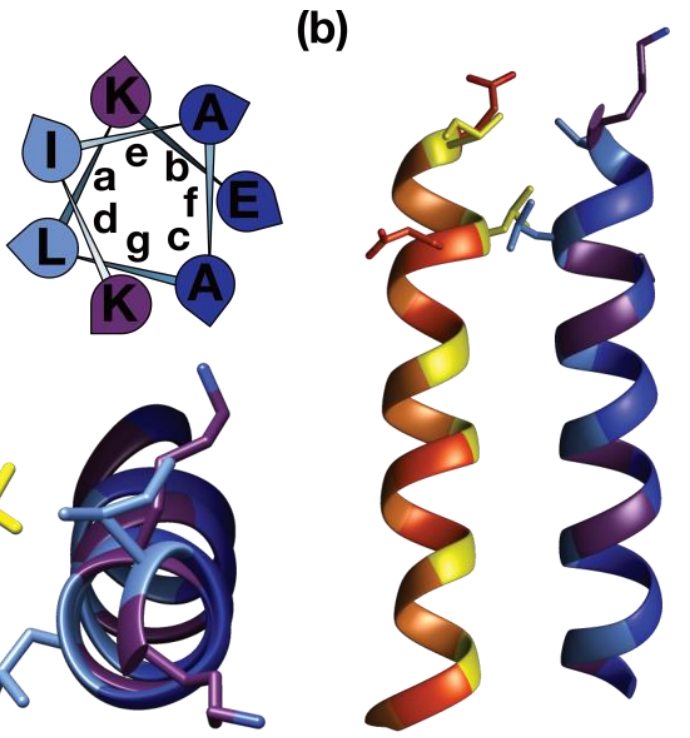

Figure 2.13: Illustration of the E3/K3 coiled coil. (a) Helical wheel depicting a heptad repeat of the sequences. (b) E3/K3 coiled coil side view. (c) Top view of the peptides. NMR solution structure from LINDHOUT et al. ${ }^{[196]}$ Molecular graphics and analyses were performed with the UCSF Chimera package. ${ }^{[121]}$ 
The E3/K3-PEG-Lipid anchor model system is capable of fusing lipid membranes and has been studied extensively regarding the fusion mechanism. ${ }^{[197]}$ As the membrane anchor, several lipids have been tested with cholesterol being the most efficient in facilitating fusion. ${ }^{[198]}$ However, it has also been observed that the secondary structure of the motif changes depending on the type of lipid anchor. ${ }^{[197]}$ Cholesterol bound species showed a more pronounced helicity indicating a more extensive interaction with the membrane. The effect of the distance between motif and membrane was also tested with this model system by attaching different lengths of PEG spacers to the two molecule species. Here, the specific mechanism of this analog can be observed, as the different roles of the two molecules become more evident. The lack of a spacer prevented fusion, whereas a too long spacer lessened the fusion efficiency. ${ }^{[197]}$ More interestingly, the combination of asymmetric spacer lengths in the K lipopeptide (LPK) and the E species (LPE) showed that LPK is much more sensitive to variations in the linker length and determines the overall fusion efficiency. The authors concluded that both lipopeptides serve different functions in the process. The E-lipopeptide acts as a handle in such a way, that it extends into the aqueous surrounding and offers its motif to bind LPK (see Figure 2.14). This is supported by experiments which modulated the membrane pressure and showed that LPE rather forms random coils than to interact with the membrane. ${ }^{[199,200]}$ LPE is also forming weak homodimeric coiled coils in aqueous environment. On the other hand, LPK showed strong interactions with the membrane which was shown by FTIR and temperature dependent CD spectroscopy. ${ }^{[201]}$ This observation can be explained by lysines ability to interact with the polar headgroups of the lipids by snorkeling into it. ${ }^{[202]}$ This K3 interaction with the membrane has a destabilizing effect, as lipids with smaller headgroups e.g. DOPE tend to be recruited by this. ${ }^{[197,203-205]}$ Consequently, the membrane is more prone to fuse as the destabilization reduces the energy needed for the merger.

It was concluded, that the LPE motif grabs onto $\mathrm{K} 3$ and transfers it to the other membrane. The interaction of $\mathrm{K} 3$ destabilizes both membranes which leads to the formation of protrusions. At this point, the distance between the liposomes is minimal which subsequently leads to fusion. ${ }^{[197]}$ 


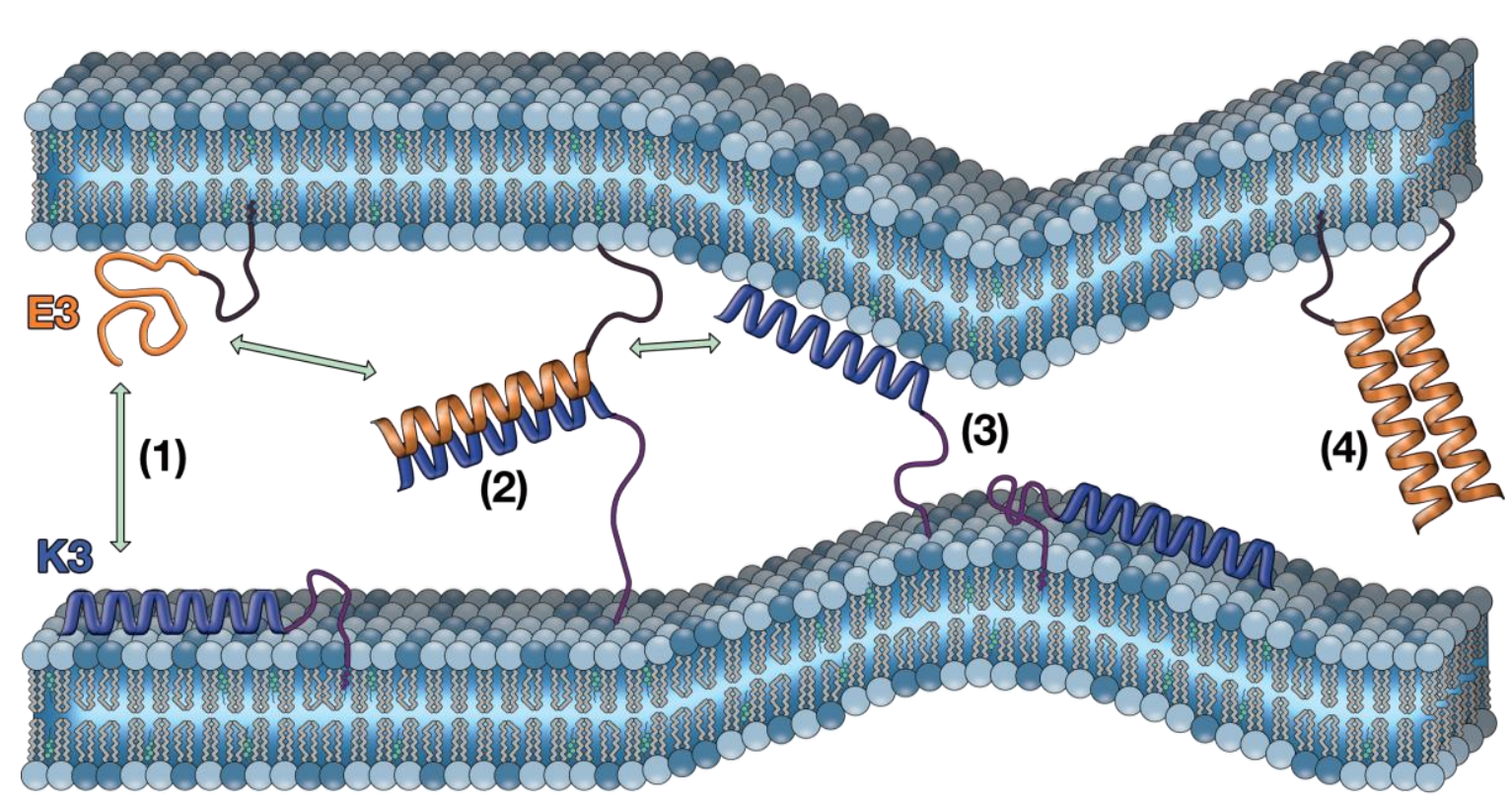

Figure 2.14: Schematic representation of the fusion behavior of LPE and LPK by DAUDEY et al. ${ }^{[197]}$ (1) E3 acts as a handle to recruit the membrane bound K3. (2) Coiled coil formation of E3/K3. (3) K3 is transferred to the opposing membrane. Destabilization of both membranes by K3 promotes fusion. (4) E3 is also forming weak homodimeric coiled coils. Based on ${ }^{[197]}$.

\subsubsection{E3/K3-TMD Model System}

With the E3/K3 coiled coil being established as a good alternative motif to the comparably big SNARE recognition unit, it was incorporated into a similar SNARE alternative. The E3/K3-TMD model system is a combination of two peptides which are designed to mimic the function of the natural SNARE core complex. ${ }^{[6]}$ It was first introduced by MEYENBERG et al. and tested upon its fusion capabilities. ${ }^{[22,206]}$ Both peptides consist of an artificial motif and a native linker and transmembrane domain as illustrated in Figure 2.15. The unique feature of this system is the incorporation of the native amino acid sequences into the design. Additionally, the artificial part is of peptidic nature which makes the transition between the two seamless. In comparison to other SNARE analogs the topology resembles more closely the native one. This has the advantage of being able to form $\alpha$-helical structures throughout the whole peptide. Both the peptides E3-Syb and K3-Sx contain 30 amino acids of the natives TMD and linker. Therefore, not all amino acids of the designated linker region of the natural proteins are included in this mimetic. 


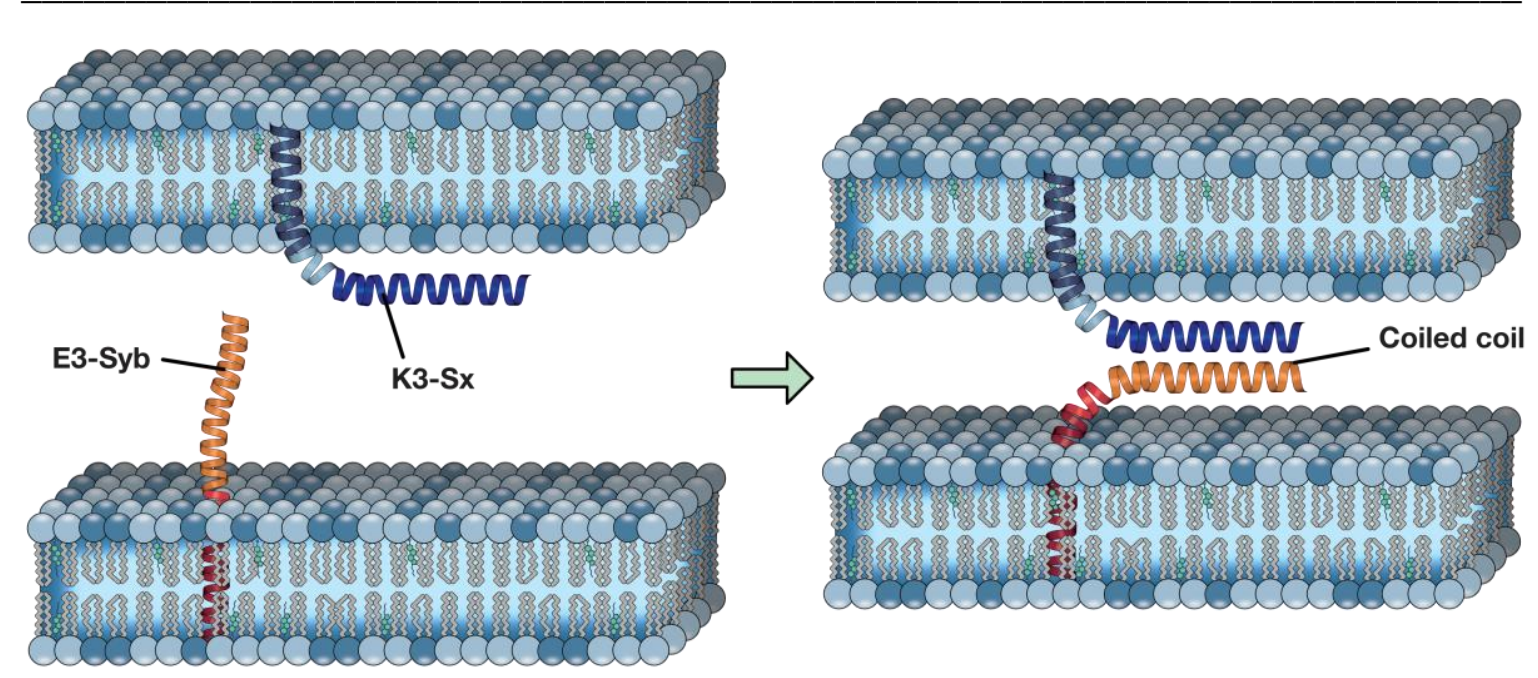

Figure 2.15: Illustration of the E3/K3-TMD system by MEYENBERG et al. ${ }^{[22]}$ The peptides E3-Syb (orange/red) and K3-Sx (blue) reside in opposite membranes. Formation of a coiled coil initiates fusion of both membranes.

As the artificial motif, the E3/K3 coiled coil pair has been chosen due to its simplicity, high stability and it being well characterized. ${ }^{[206]}$ Additionally, the coiled coil was already successfully used for vesicle fusion in a similar system (see section 2.7.3). ${ }^{[19]}$ A side effect of the artificial motif K3 is its ability to adhere to membranes and destabilize them. ${ }^{[197,202]}$ This feature differs from the natural system and has yet to be investigated in more detail.

One advantage of the E3/K3-TMD system is the high potential of modification. In fact, due to its peptidic nature and the current state of peptide synthesis methods, alterations are very easy and reliable. On the other hand, a disadvantage of the model system is the amphiphilic character of the peptides. The hydrophilic motif and the lipophilic TMD make the purification quiet challenging. For this reason, up until now, experiments performed with this system could not be conducted with high peptide quality. ${ }^{[206,207]}$

Nevertheless, the system is capable of performing full fusion as indicated by lipid and content mixing assays. ${ }^{[22]}$ Furthermore, an increase of the K3-Sx linker length showed a reduction in fusion efficiency and especially lowered the amount of content mixing. ${ }^{[206]}$ Similar to the PNA-TMD model system (see section 2.7.2), differently charged C-termini have been introduced into the E3/K3-TMD peptides. ${ }^{[207]}$ The exchange of the carboxy group with an amide yielded the most fusogenic system, whereas additional negative charges reduced overall fusion efficiency. Furthermore, antiparallel variants of E3-Syb and K3-Sx have already been synthesized and their fusion behavior studied in lipid mixing 
assays. This orientation significantly hampered with the systems ability to perform fusion and is in line with the expectations considering the natural machineries setup.

Considering all the above, the E3/K3-TMD model system offers many possibilities of modification, easy synthesis and high potential regarding its comparability to the natural system. Due to these advantages the model system has been chosen for this works investigations. 



\section{Detection of Liposome Fusion Events}

Over the years many different methods of monitoring liposome fusion in vitro have been developed. ${ }^{[208]}$ Techniques ranging from bulk assays with many liposomes fusing simultaneously to the observation of single vesicle fusion events are used for this purpose. ${ }^{[209,210]}$ Most of these methods use a fluorescence-based approach, where a fluorophore is incorporated either into the membranes or the peptides itself. Fusion can then be observed by monitoring changes in the fluorescence intensity using a microscope or spectrometer. During the fusion process, different stages are passed (e.g. docking or hemifusion, see section 2.3) which can be distinguished using some of these methods.

Many of the techniques use two fluorophores which form a Förster resonance energy transfer (FRET) pair. ${ }^{[211]}$ FRET is a physical phenomenon where energy is transferred nonradiatively between a donor and acceptor fluorophore. ${ }^{[212]}$ The donors emission spectrum overlaps with the acceptors excitation spectrum. The energy transfer is very sensitive to the distance between the two molecules as depicted in equation 2.1.

$$
\eta_{F R E T}=\frac{1}{1+\left(\frac{r}{R_{0}}\right)^{6}}
$$

The FRET efficiency $\eta_{F R E T}$ is a result of the distance $r$ between the fluorophores and the Förster radius $\mathrm{R}_{0}$, a value describing the distance where $50 \%$ efficiency is reached between the specific fluorophores. FRET is most efficient between the ranges of 1-10 nm. ${ }^{[213]} \mathrm{In}$ the following chapters, fusion monitoring methods are described which were used in this work.

\subsection{FRET Dequenching Assay}

FRET dequenching assays are bulk fusion detection methods where total lipid mixing (TLM) is observed using a FRET pair. The donor fluorophore $N$-(7-nitro-2-1,3benzoxadiazol-4-yl) (NBD) and the acceptor dye Lissamine rhodamine B (Rh) are both introduced into the same liposome species (see Figure 3.1). Both are connected to a lipid head group e.g. DOPE and embedded in an appropriate concentration inside the membrane. 
Due to the fluorophores being in close proximity within the FRET range (see equation 3.1), excitation of the donor leads to the energy transfer and light emission of the acceptor. FRET can be reduced by increasing the distance between the dyes in the vesicle. This can be achieved by the merger with a liposome lacking fluorophores. As the average distance between the FRET pair increases, the acceptor emission decreases. This results in an increase of the donor emission during its excitation.

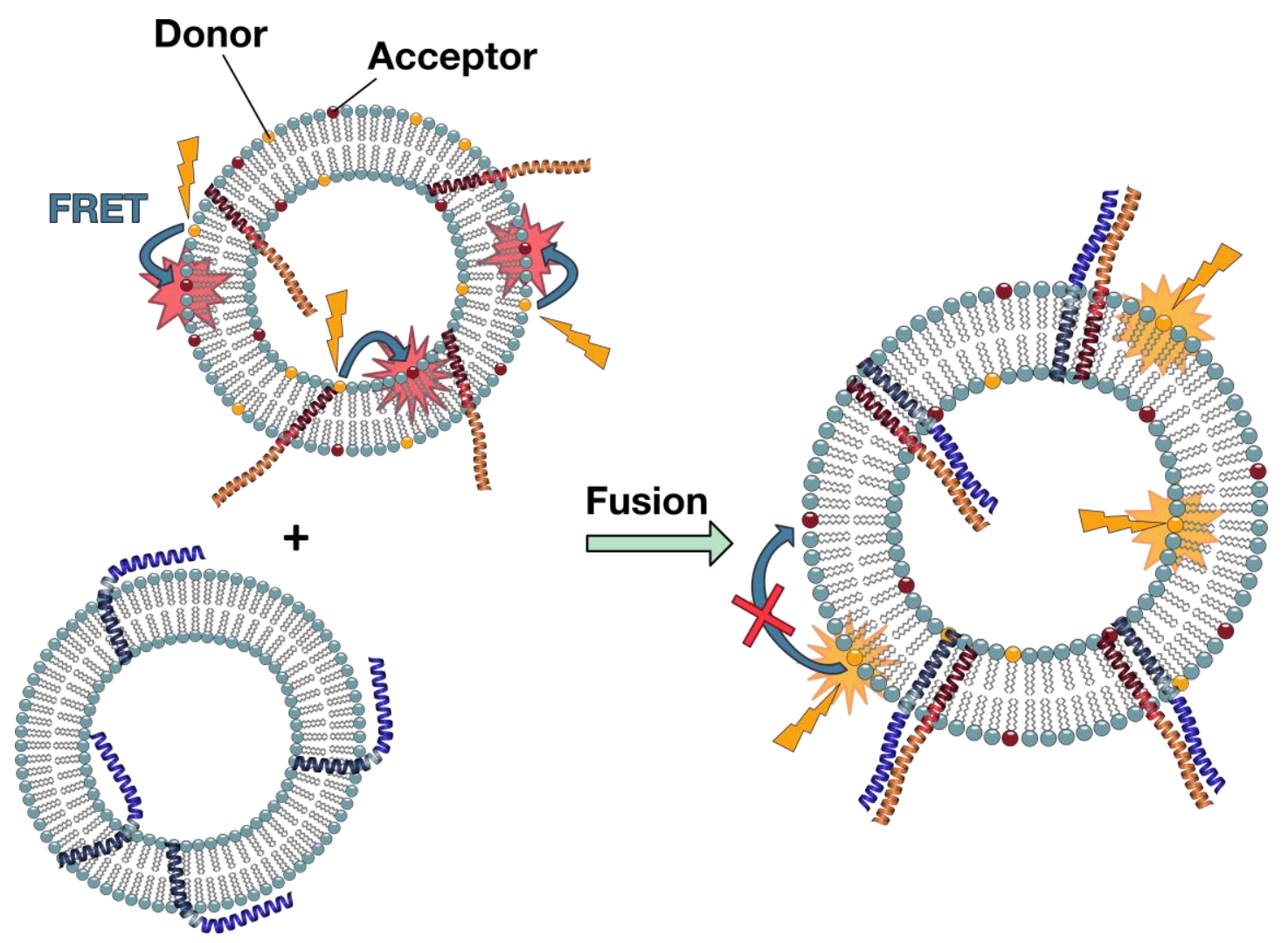

Figure 3.1: Illustration of FRET dequenching assay. Two liposome species are decorated with different peptides. The labeled liposome contains both fluorophores and the E3-Syb peptide. Due to the small distance between the fluorophores, FRET occurs, which leads to the emission of light by the acceptor. The unlabeled liposome only contains the K3-Sx peptide. After fusion, the concentration of the fluorophores decreases and the distance between the molecules increases. FRET can no longer occur and the donor emits light.

However, this method cannot differentiate between full and hemifusion, as the fluorophores are located in the inner and outer leaflet. To distinguish between the two, NBD of the outer leaflet can be destroyed e.g. through oxidation. Consequently, an increasing NBD emission can only be due to inner leaflet mixing (ILM). 


\subsection{Dynamic Light Scattering}

An alternative method of detecting fusion between liposomes is dynamic light scattering (DLS). With DLS the sizes of the liposomes can be monitored. Comparing sizes of the initially used liposomes with the population post fusion is a direct way of observing fusion or aggregation. Using DLS to determine the size increase has been established in the past decades. ${ }^{[214-217]}$

The technique uses monochromatic light which is directed at the sample. The light is then scattered depending on the size and shape of the macromolecules. ${ }^{[218]}$ Additionally, the intensity fluctuates due to Brownian motion is measured. The particle size is one of the defining properties responsible for the fluctuation frequency. The smaller the particle, the faster it moves. The fluctuations of the scattered light are recorded and analyzed according to the Stokes-Einstein relationship in equation 3.2.

$$
d_{\mathrm{h}}=\frac{k_{\mathrm{B}} T}{3 \pi \eta D}
$$

The hydrodynamic diameter $\mathrm{d}_{\mathrm{h}}$ is calculated using the Boltzmann constant $k_{\mathrm{B}}$, the absolute temperature $T$, the viscosity of the solvent $\eta$ and the diffusion coefficient $D$. The coefficient is calculated out of the decay rate, which is gathered during the autocorrelation analysis of the fluctuations. The equation applies only to spherical particles, which makes the interpretation of other shaped pieces challenging. Furthermore, the hydrodynamic diameter includes the core particles as well as anything bound to the surface e.g. ions and the hydration sphere. The measured size is therefore always larger than its actual size. The hydrodynamic radius is often presented as a Z-average value, which is the intensity weighted mean of the measurement.

An additional value obtained with this method is the polydispersity index (PdI), which describes the heterogeneity of the measured sample based on size. The larger the number, the more heterogenic the sample. 



\section{Optimization of Fusion Assays}

In this chapter, the experimental setup and parameters are investigated thoroughly to improve upon previous difficulties in the analysis of the fusion behavior of the E3/K3-TMD model system. In section 4.1, the applied experiments are described and introduced refinements are discussed. Section 4.2 focusses on the synthesis of the peptides used in this work. Here, different methods were tested and evaluated to improve the overall purity of these peptides. Finally, part 4.3 is about multiple optimization approaches in the different experimental steps of FRET dequenching assays. The resulting effects and outcomes are presented and discussed regarding their benefits.

\subsection{Fusion Assays}

The main measurement technique employed in this work were FRET dequenching assays. As described in chapter 3.1, two different liposome species were used. The one including the FRET pair (labeled liposome) was either not containing any peptide or exclusively a E3-TMD peptide variant. The unlabeled vesicles were always decorated with a K3-TMD if a peptide was used.

\subsubsection{Liposome Preparation}

The liposomes were prepared by combining all components in small glass tubes (detailed description in chapter 9.4). The lipids and peptides were stored as stock solutions in chloroform or TFE at $-20^{\circ} \mathrm{C}$. Lipids were additionally stored under an argon atmosphere to reduce the amount of oxidation. In order to minimize evaporation of the solvents, the solutions were kept at $0{ }^{\circ} \mathrm{C}$ during usage and only briefly exposed to ambient air for withdrawal. After combining of all components in the glass tube, the samples were vortexed for $5 \mathrm{~s}$ and heated to ambient temperature. As TFE and chloroform are immiscible at $0{ }^{\circ} \mathrm{C}$, the samples were vortexed a second time for $5 \mathrm{~s}$ to ensure even mixing. Next, the solvent was evaporated at $50{ }^{\circ} \mathrm{C}$ using a nitrogen stream. A lipid film formed in the bottom part of 
the glass tube which was dried overnight in vacuo. Lipid films were generally used one day after preparation. Rehydration was achieved by using HEPES (4-(2-hydroxyethyl)-1piperazineethanesulfonic acid) buffer and small glass beads at a temperature of $40{ }^{\circ} \mathrm{C}$ while stirring for at least $2.5 \mathrm{~h}$. During this time, the formation of multilamellar vesicles (MLVs) occurred by the detachment of the lipid films from the glass wall. Prior to extrusion, the solution was vortexed for $5 \mathrm{~s}$, exposed to sonication for a maximum of $10 \mathrm{~s}$ and vortexed again for $5 \mathrm{~s}$ to yield a fine cloudy solution. The solution was extruded through a polycarbonate membrane to yield large unilamellar vesicles (LUVs) with diameters ranging from 120-150 nm. Lipid loss was determined for E3-Syb peptide containing vesicles to be $\sim 32 \%$ and K3-Sx containing vesicles at $\sim 45 \%$. These values were highly dependent on the used peptide and also varied in between same peptide species. K3-Sx containing vesicles were always extruded last, as the stability is lower compared to the other used vesicle species. The time between extrusion and start of the measurements for each measurement was below $5 \mathrm{~min}$, eliminating effects which could occur in case of aggregation of the vesicles over a period of time.

\subsubsection{Lipid Mixing Assays}

The measurements were performed at $25^{\circ} \mathrm{C}$ in HEPES buffer to ensure comparability to previous experiments. ${ }^{[22,188,207,219]}$ To the buffer ethylenediaminetetraacetic acid (EDTA) was added to catch present $\mathrm{Ca}^{2+}$-ions, which are known to be involved in the natural SNARE mediated vesicle fusion (see section 2.6.3). Effects due to the ion are inhibited in presence of EDTA. Additionally, dithiothreitol (DTT) was added to prevent disulfide bridge formation of cystein residues by oxidation.

Primarily, FRET dequenching assays were performed using the FRET donor 7-nitro-2-1,3benzoxadiazole (NBD) and acceptor Lissamine Rhodamine B (Rh). These labels were also used in previous experiments which increases comparability between these results. ${ }^{[188,206,207]}$ The use of Oregon Green 488 (OG) and Texas Red (TR) was also tested, but did not show significant benefits compared to NBD and Rh. In fact, handling these labels was more challenging, as they are more sensitive to ambient light and are therefore more prone to photo bleaching. In addition to the dequenching assays, FRET quenching assays were also performed for a few samples. However, the obtained results were not consistent and reproducible and consequently not included in this work. 
Vesicles containing E3-Syb peptides were prepared containing the lipidic fluorophores whereas K3-Sx vesicles were always unlabeled. As described in section 4.3.3, it cannot be ruled out, that the fluorophores interact with the peptides. The interaction is small but is nevertheless being taken into account by the use of the right control measurements (see section 4.3.4). Stability of the vesicles was the main complication in previous experiments. ${ }^{[207]}$ Indeed, K3-Sx vesicles are much more prone to aggregation and lipid loss during extrusion. But the optimizations employed to the FRET dequenching assays improved vesicle stability significantly (see section 4.3).

\subsubsection{Dynamic Light Scattering Experiments}

Dynamic light scattering (DLS) experiments were performed for each single vesicle population as well as with the mixed populations after the FRET dequenching assays (for a more detailed description see chapter 9.4). Measurements for different vesicle species were done within $5 \mathrm{~min}$ after extrusion. The exception being the mixed population, which was measured directly after the $20 \mathrm{~min}$ fusion assay finished. With this approach, the vesicle sizes at the start of the dequenching assays could be determined consistently. For the measurements, part of the vesicle sample was diluted with buffer. The reason for this was twofold. A too high concentration of particles in the DLS sample can lead to multiple light scattering. ${ }^{[218]}$ This phenomenon occurs when a photon is scattered by more than one particle. The result is a reduction in the particle size measured. Second, the DLS and fusion assay measurements could be performed simultaneously. The times between extrusion, DLS and dequenching assay measurements were reduced to a minimum. This way, the sizes of the vesicles conform to both experiments, ruling out time dependent alterations of the sample. The measured sizes differed for the different peptide species as illustrated in Figure 4.1.

Generally, E3-Syb containing vesicles were smaller compared to K3-Sx vesicles. Liposomes without peptides were about the same size as E3-Syb vesicles with a diameter intensity mean (Z-average) of $\sim 125 \mathrm{~nm}$. K3-Sx vesicles were usually measured with $\sim 135 \mathrm{~nm}$ and mixed vesicles with $\sim 145 \mathrm{~nm}$. The value for the mixed vesicles is a composition of the two used vesicle species and fused vesicles. The increased size of the particles after mixing indicates that the vesicles either fused or aggregated. In conjunction with the fusion assays as well as with previous results using this model system, ${ }^{[22]}$ the size 
increase is believed to be due to vesicle fusion rather than aggregation. Size increase was only observed in case of a positive FRET dequenching assay result.

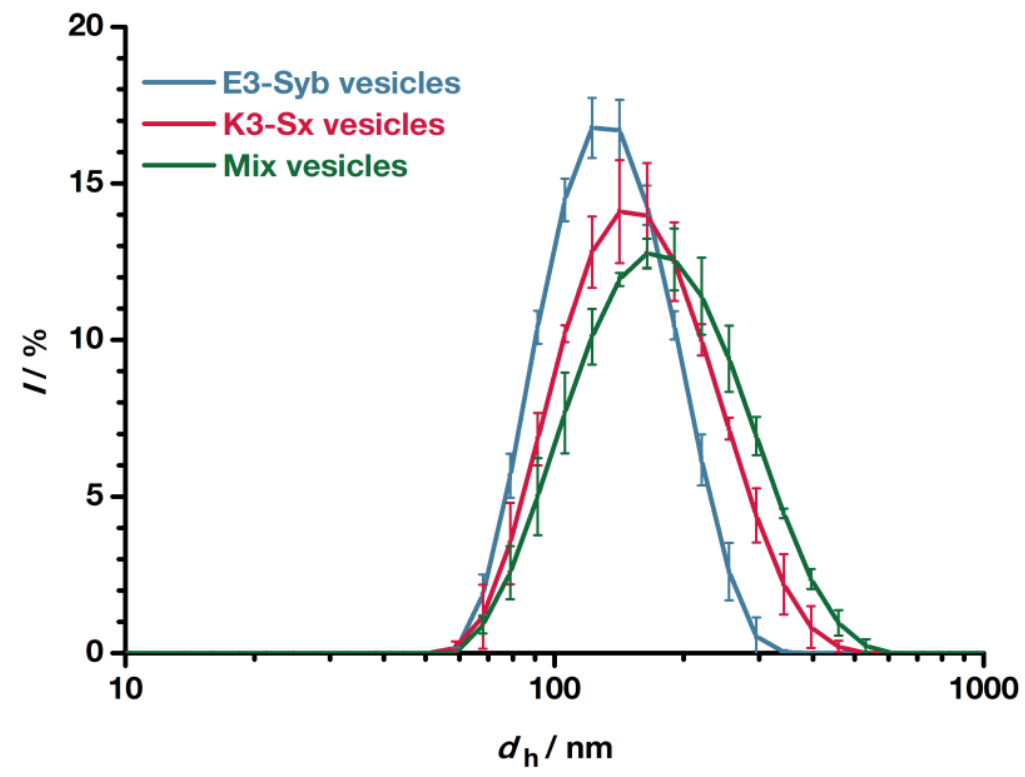

Figure 4.1: Exemplary DLS data of the three different vesicle populations. (blue) Vesicle population containing E3-Syb peptides and the FRET fluorophores. (red) Vesicles with K3-Sx peptides. (green) Mixed population consisting of E3Syb, K3-Sx and fused vesicles.

The relatively small size increase can be explained with the stoichiometry of the fusion. In the case of E3-Syb vesicles having a size of $125 \mathrm{~nm}$ and K3-Sx vesicles $135 \mathrm{~nm}$, the theoretical size can be calculated using the volume of a sphere with the following equation: $V=1 / 6 \pi d^{3}$. After summation of both volumes, the diameter of the fused vesicle can be calculated yielding $164 \mathrm{~nm}$. This implies a fusion ratio of K3-Sx/E3-Syb $=1: 1$. However, the volume ratio at which the two vesicle species are mixed is K3-Sx/E3-Syb $=4: 1$. Therefore, after the hypothetical complete fusion, two kinds of vesicles are left in the sample. Three parts of K3-Sx vesicles and one part of the fused species. Calculating the mean diameter of these four vesicles yields $\sim 142 \mathrm{~nm}$. This diameter lies in the size range of the measured mixed species. Of course, this explanation attempt has to be treated very cautiously, as it leaves out many other plausible liposome species and other factors. For example, fused liposomes resulting of more than one fusion event, or docked liposomes are not factored in. Moreover, as the FRET dequenching assays show, not all vesicles are involved in fusion events. The majority of particles in the sample remain sized like the initial two species. With these caveats in mind, the size increase is expected to be small, rather than to show a significant difference. Additionally, smaller sizes indicate that no 
aggregates are formed, which in return can help to draw conclusions about the used model system. Consequently, the DLS data acquired has to be accompanied by other measurements for interpretation. Nevertheless, the technique can give more information about the fusion experiment and especially the quality of the vesicles used (see also section 4.3.2).

\subsection{Peptide Synthesis}

The E3/K3-TMD model system is a combination of an artificial motif connected to the native linker and transmembrane domain (TMD) of synaptobrevin II and syntaxin 1A. The motif consists of the well-studied E3/K3 coiled coil pair. ${ }^{[21,220-222]}$ The model system has been developed earlier and was already tested for its fusion capabilities. ${ }^{[22,184,206]}$ Synthesis of all peptides was performed via automated microwave assisted solid phase peptide synthesis (SPPS). Briefly, SPPS uses a solid support onto which the peptide is bound covalently and is elongated with consecutive couplings. ${ }^{[223]}$ Excess reagents and other solvable side products can be removed easily via filtration. The synthesis utilizes an orthogonal protecting group strategy and optimized reagents for high yields and purity.

The syntheses were performed on a total of three different synthesizers. Two of which were of the same model but used different methods for coupling. Generally, the same reagents were used for all synthesizers, but different coupling times, concentrations and temperatures were applied. As solvent dimethylformamide (DMF) was used for all synthesizers. The specific reaction conditions for every machine are listed in section 9.3. For the solid support a low loaded Wang resin, preloaded with the first amino acid, either glycine or threonine, was used. The low load density $(0.28-0.36 \mathrm{mmol} / \mathrm{g})$ makes the individual peptides spatially more available and reduces aggregation of the peptides. The resins were equipped with sufficient swelling properties, enhancing the handling and reducing swelling times prior to synthesis. After cleavage, the peptides were left with a carboxylic acid at the C-terminus. The effect of different C-terminal groups and amino acids was previously studied with the E3/K3-TMD and PNA-TMD model system. ${ }^{[184,207]}$ To stay close to the natural system, no additional modifications have been made in the TMDs of both peptide species. 
Deprotection of the N-terminal fluorenylmethyloxycarbonyl (Fmoc) group was achieved with $20 \%$ or $25 \%$ piperidine in DMF. The reaction was heated to $90{ }^{\circ} \mathrm{C}$ for $90 \mathrm{~s}$ to maximize the deprotection rate. After deprotection, the next amino acid was coupled onto the peptide. The coupling method was chosen following three criteria. First, which synthesizer was the peptide coupled on. Second, which kind of amino acid was coupled. For arginine, cysteine and modified amino acids, specialized methods were used to reduce side reactions and improve coupling efficiency. Third, the position of the amino acid in the peptide sequence was considered. The first 30 amino acids were coupled once, whereas all subsequent amino acids were coupled twice. This was done to maximize coupling efficiency and reduce the amount of undesired peptides with errors in the sequence due to omission. Coupling was facilitated with commercially available amino acids (5 eq.), the activator $N, N^{\prime}$-diisopropylcarbodiimide (DIC) (5/10 eq.), Oxyma Pure (5 eq.) as an additive to prevent racemization and DIPEA (0.5 eq.) to reduce formation of precipitates of the reagents. Coupling times and temperatures differed between the individual synthesizers. Most peptides were coupled at $90{ }^{\circ} \mathrm{C}$ for $4 \mathrm{~min}$. However, the best results were obtained using the CarboMAX method which was developed by $C E M .^{[224]}$ Here, an increased amount of DIC (10 eq.) and an elevated temperature of $105^{\circ} \mathrm{C}$ were used. This method decreased the overall needed time and yielded better HPLC separation results and less impurities in mass spectra of the raw peptide.

Cleavage from resin and deprotection of all protecting groups of the amino acid side chains was achieved simultaneously with a mixture of TFA/TIS/EDT/ $\mathrm{H}_{2} \mathrm{O}(95: 2: 2: 1, v / v / v / v)$. TIS and $\mathrm{H}_{2} \mathrm{O}$ were used to capture reactive products and EDT to prevent disulfide formation between cysteine residues. Prior to HPLC purification, the raw peptide was precipitated multiple times in ice cold diethyl ether and resolubilized in HFIP and water to get a uniform powder after lyophilization.

All in all, the synthesis of the peptides was improved due to the use of optimized synthesis methods. The peptide quality before purification was increased, which is beneficial for all consecutive steps e.g. FRET dequenching assays. 


\subsection{Enhancement of FRET Dequenching Assays}

In previous experiments FRET dequenching assays conducted with K3-Sx and E3-Syb revealed many obstacles. Especially, the stability of K3-Sx vesicles posed a challenge in the preparation and use during measurements. ${ }^{[207]}$ The main problem has been identified as an aggregation process of the vesicle solution pre and post extrusion. The aggregation was monitored with a variety of techniques and was also visible for the naked eye as the formation of precipitant would occur within only a few minutes. Vesicles containing no peptides, either labeled or unlabeled, did not show any kind of aggregation. This lead to the conclusion, that the used peptides are the main factor of this event. To improve upon the above-mentioned challenges, several tests have been performed in this thesis.

The following experiments were done using the peptides shown in Table 4.1. The combination of E3-Syb(RKY) and K3-Sx(QSK) is referred to as the reference system in this work. The peptide sequences match the ones originally introduced by MEYENBERG. ${ }^{[22,206]}$

Table 4.1: Peptides used for the optimization process. The sequences of the transmembrane domains and linker (bold) resemble the ones of the natural proteins synaptobrevin 2 and syntaxin $1 \mathrm{~A}$.

$\begin{array}{lll}\text { Peptide Sequence } & \text { No. }\end{array}$

E3-Syb(RKY) G-(EIAALEK) $)_{3}-$ RKYWWKNLK-MMIILGVICAIILIIIIVYFST-OH (1)

K3-Sx(QSK) WWG-(KIAALKE) ${ }_{3}$-QSKARRKK-IMIIICCVILGIIIASTIGGIFG-OH (2)

\subsubsection{Peptide Purity as a Factor for Fusion}

In previous works, the decrease of vesicle concentration from $5 \mathrm{nM}$ to $1.3 \mathrm{nM}$ yielded more stable vesicles, as well as an increase of fusogenicity. ${ }^{[188]}$ These advancements were achieved using a similar model system consisting of an aegPNA motif attached to the natural TMD. These improvements were implemented in this thesis using the E3/K3-TMD system. However, this complex behaves quite differently and therefore additional adjustments had to be made.

To gather more consistent and reliable data in experiments where peptides are used in vesicles, the production of these vesicles had to be optimized. The first approach was to use peptides of a higher purity. In previous experiments, the purification of the E3-Syb and K3-Sx was not successful. ${ }^{[206]}$ After more research and optimization, these peptides could 
be purified successfully (for more details, see chapter 9.2.7). Solvation of the raw peptides in HFIP and water before elution upon a HPLC column yielded a significant increase in peptide purity (See Figure 4.2). Each peptide used for this thesis was successfully purified and its quality verified via UHPLC and high-resolution mass spectrometry.

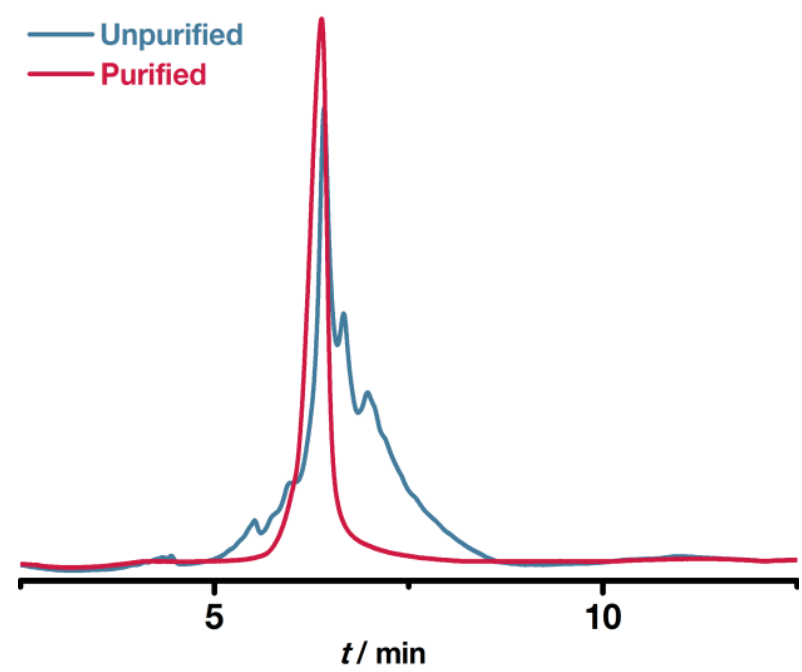

Figure 4.2: UPLC chromatograms of a raw/unpurified peptide and the same peptide after HPLC purification. Parameters: ACE Excel 2, 100 A $2 \mu \mathrm{m} \mathrm{C18,}$ gradient $70-99 \% \mathrm{~B}$ in $8 \mathrm{~min}, 50{ }^{\circ} \mathrm{C}, 215 \mathrm{~nm}$. A: water $+0.1 \%$ TFA; B: $\mathrm{MeOH}+0.85 \%$ TFA.

Intuitively, purified peptides should be capable of fusing LUVs more efficiently and effectively, as no impurities should interfere with the pure peptides. In addition, determination of peptide concentration should be more accurate as a consequence of the reduced number of break-off peptide sequences. This entails a higher number of peptides capable of performing the fusion process. According to these assumptions, unpurified peptides should yield a lower signal and a slower increase in dequenching FRET assays. Surprisingly, the exact opposite effect was observed in multiple experiments as seen in Figure 4.3. After 20 min the disparity in the absolute signal is about $6 \%$ which translates to a relative difference of $40 \%$ between the curves. Furthermore, in the first $200 \mathrm{~s}$ a noticeable contrast in kinetics, regarding the slope is present. From these observations, it can be concluded that the vesicle species with unpurified peptides fuse more rapidly as well as more effectively, leading to a higher total signal. These findings contradict the initial predictions regarding the fusion behavior. 


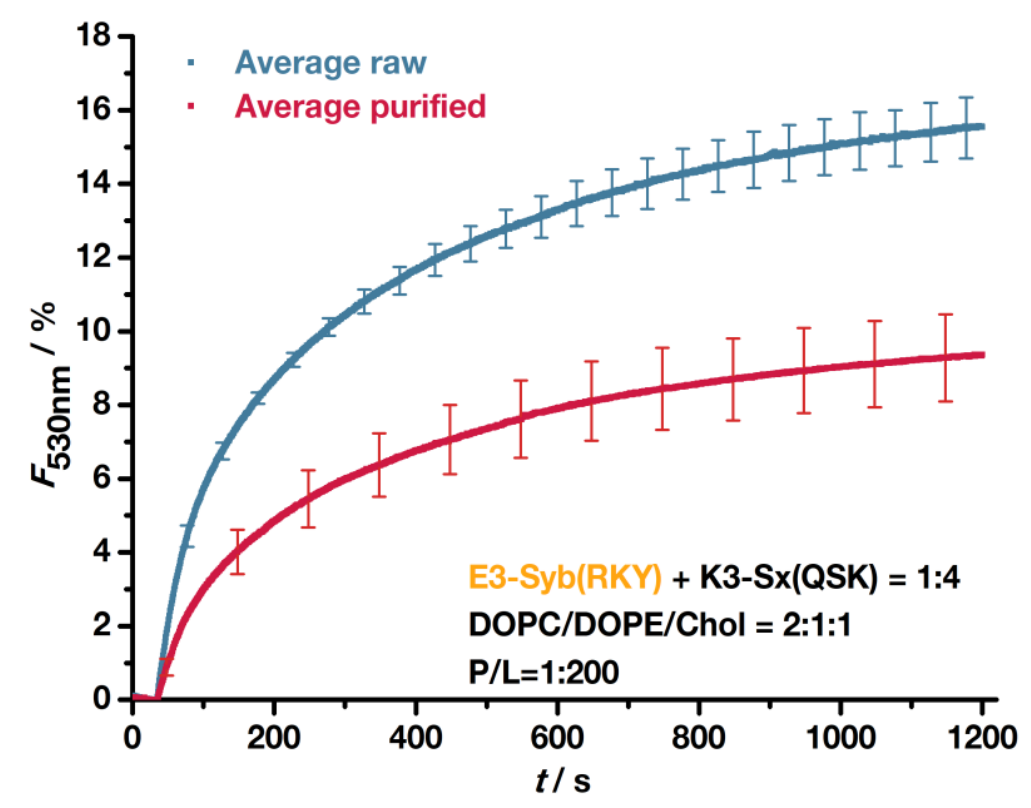

Figure 4.3: FRET dequenching assay of the reference system with a peptide/lipid ratio of 1:200. The effect of using HPLC-purified peptides (red) in comparison to unpurified peptides (blue) is shown. The curves represent the average of at least three measurements and the error bars show the standard deviation.

Apparently, break-off peptide sequences and other impurities have a different effect on the fusion process as previously thought. A reason for this observation could be that the impurities destabilize the vesicle membranes, reducing the energy needed for fusion. Another approach to explain this discovery, is that the lack of impurities leads to more homomeric interactions with the completed peptides. For example, K3 is capable of forming homodimeric structures, albeit the percentage of peptides following this behavior is quiet low. ${ }^{[25]}$ In combination with observations of syntaxin $1 \mathrm{~A}$ clustering in membranes, ${ }^{[226-229]}$ it is possible that the interaction between the peptides is more prominent without impurities. A stronger homomeric binding behavior entails less peptides which are engaged in the fusion process. Furthermore, kinetics can change as a result of the new formed complex of K3-Sx, reducing the speed of fusion. One more reason for the higher signal of the unpurified species can be related to the peptide concentration. Especially in case of K3-Sx, a big discrepancy in the number of peptides in the vesicles is possible. The tryptophan residues used for the determination of the concentration are located at the $\mathrm{N}$-terminus and are therefore coupled last. The coupling efficiency decreases with the length of the peptide, making it more likely to produce peptides with just one or even without tryptophan residues. ${ }^{[230]}$ In the end, the tryptophans are not important for the interaction of the E3/K3 motif (see chapter 2.7.3), making them capable of performing the 
fusion. However, the tryptophan residues are vital for determining the concentration of the stock solution.

To summarize, these results show that the impurities increase the FRET intensity measured, rendering peptide purification as an essential step to obtain more accurate results. Without purification, more peptides capable of performing the necessary interactions are used in the experiment than intended, leading to a higher total signal. Additionally, the stability of the vesicles was improved as no precipitant formed within a few hours after extrusion. However, a polymodal particle solution could be measured via DLS after a few minutes, indicating aggregation. Further improvements had to be implemented to produce even more consistent and reliable data.

\subsubsection{Peptide to Lipid Ratio}

The use of purified peptides in the dequenching FRET experiments lead to a decrease in total fusion efficiency. However, the loss of intensity was accompanied with more reliable data and a deeper understanding of the role of impurities in this process. After all, the main complication in preceding experiments was the stability of the K3-Sx vesicles. Although using purified peptides did not solve this problem, a significant improvement in data quality was achieved. The next approach focused on the number of peptides included in the vesicles.

Determining the quality of the vesicles was achieved by DLS measurements (see also section 4.1.3). A monomodal sample with a small Polydispersity index (PdI) is a good indication whether the used vesicles are stable and can therefore be used for the FRET dequenching assays. As illustrated in Figure 4.4, E3-Syb and labeled vesicles without peptides show a narrow distribution with an average diameter of $125 \mathrm{~nm}$ and $124 \mathrm{~nm}$ respectively. Both vesicle species maintained their size for several days and can therefore be considered as stable. K3-Sx vesicles on the other hand, showed a much bigger PdI of 0.406 , in comparison to $0.069 / 0.078$, a mean diameter of $172 \mathrm{~nm}$ and multiple peaks ranging up to $6000 \mathrm{~nm}$. In this polymodal solution the mean diameter is not a reliable value anymore. The measurement for the K3-Sx vesicle species was observed just 2 min after extrusion, concluding this vesicle species to be highly unstable and verifying previous results. ${ }^{[207]}$ 
(a)

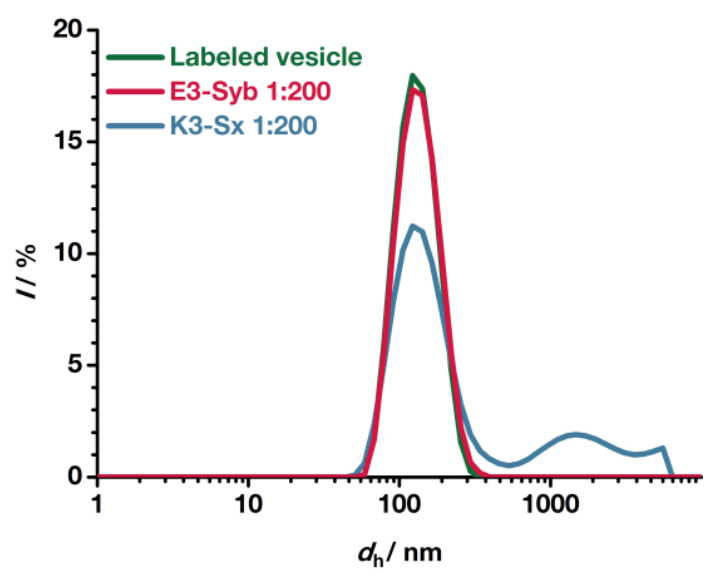

(b)

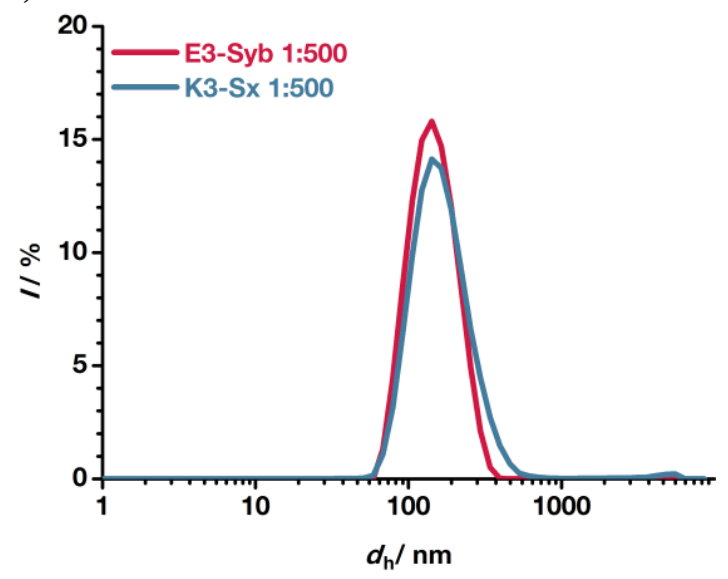

\begin{tabular}{lcc} 
Peptide & $\begin{array}{c}\text { Z-average } \\
\boldsymbol{d}_{\mathbf{h}} / \mathbf{n m}\end{array}$ & PdI \\
\hline E3-Syb(RKY) & 125 & 0.069 \\
K3-Sx(QSK) & 172 & 0.406 \\
No Peptide & 124 & 0.078 \\
\hline
\end{tabular}

\begin{tabular}{lcc} 
Peptide & $\begin{array}{c}\text { Z-average } \\
\boldsymbol{d}_{\mathbf{h}} / \mathbf{n m}\end{array}$ & PdI \\
\hline E3-Syb(RKY) & 123 & 0.047 \\
K3-Sx(QSK) & 134 & 0.083 \\
\hline
\end{tabular}

Figure 4.4: DLS data of the different used vesicle species. (red) E3-Syb vesicles. (blue) K3-Sx vesicles. (green) Labeled vesicle without peptide. (a) DLS data of vesicles used in the experiments with $\mathrm{P} / \mathrm{L}=1: 200$. (b) Vesicles used in experiments with $\mathrm{P} / \mathrm{L}=1: 500$. The labeled vesicle species is omitted, as there is no difference in the experiments due to the lack of peptides in this vesicle.

To improve on the inconsistent $\mathrm{K} 3-\mathrm{Sx}$ vesicles, a variety of changes in the preparation method have been tested. For example, the vesicles were exposed to several freeze-thaw cycles, purification of the vesicles using gel-columns and the lipid composition was changed. Despite several tests, the quality of the K3-Sx vesicles could not be improved significantly until changes in the peptide to lipid ratio were implemented. Previously, changes to the peptide to lipid ratio from 1:200 to 1:1000 yielded promising results in the PNA-TMD system. ${ }^{[188]}$ However, this ratio was not suitable for the use with the E3/K3TMD system, as the FRET dequenching assay signal were too low. The difference between the control and a measurement with the reference system in total intensity was below $1 \%$, making it impossible to detect any changes in the fusion behavior. In the end, using a $\mathrm{P} / \mathrm{L}=1: 500$ ratio resulted in positive results regarding the dequenching assay intensities as well as vesicle quality as illustrated in Figure 4.5. 


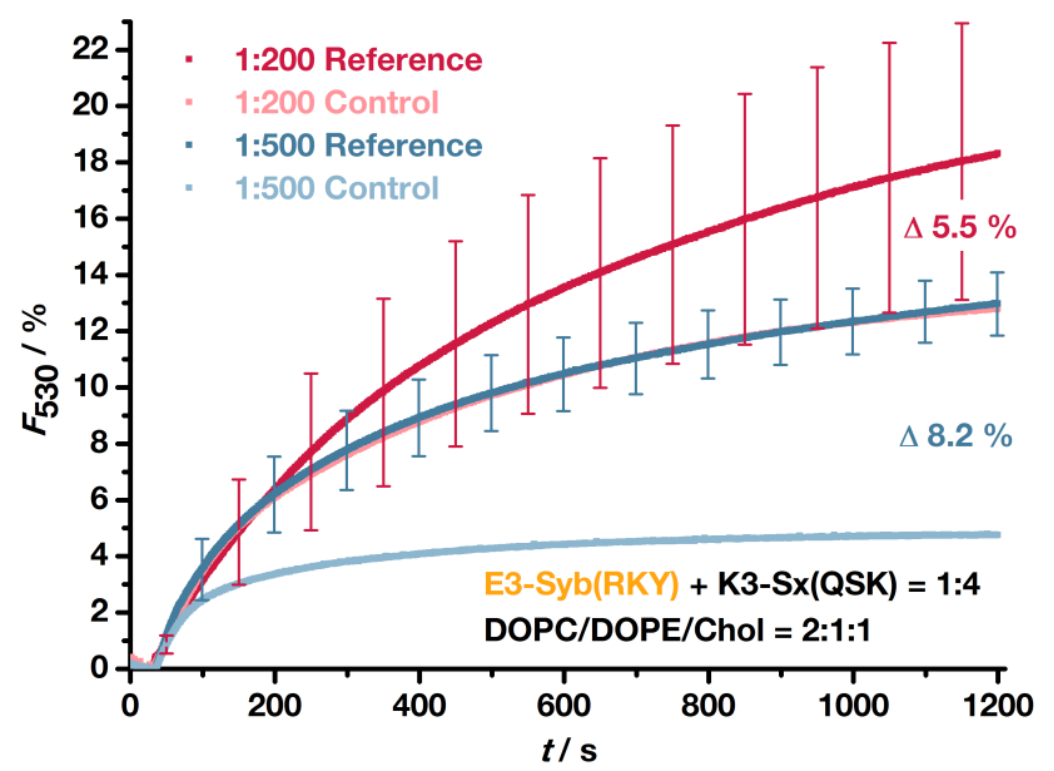

Figure 4.5: FRET dequenching assay comparison between different $\mathrm{P} / \mathrm{L}$ ratios and their corresponding control. (red) 1:200 measurements with a difference in intensity after $20 \mathrm{~min}$ of $5.5 \%$. (blue) Measurements with $\mathrm{P} / \mathrm{L}=1: 500$ showing a difference of $8.2 \%$ after $20 \mathrm{~min}$. The nearly identical curve of the 1:200 control and 1:500 reference is coincidental. Error bars for control measurements were omitted due to the comparably small error. All measurements were performed thrice.

First, the reference measurements of $\mathrm{P} / \mathrm{L}=1: 200$ show the biggest total increase after $20 \mathrm{~min}$ in signal intensity. Surprisingly, one of the measurements reached a total intensity of over $23 \%$, making it the highest recorded measurement in this work. However, this is also accompanied by the biggest mean variation as indicated by the error bars. These measurements were much more inconsistent in terms of their total signal intensity. In the first $200 \mathrm{~s}$ of the measurements, the $\mathrm{P} / \mathrm{L}=1: 200$ reference and control experiments showed very similar kinetics. This observation is quiet surprising, as the control has only one of the two needed peptides (K3-Sx(QSK)) and should therefore show completely different kinetics in the beginning. In fact, this observation leads to the conclusion that the vesicles of the control interact with each other in some way, that the fluorophores are being separated from each other. The liposomes are capable of either fusing with only one peptide present, or K3-Sx destabilizes the other vesicle species. Both scenarios are not desirable during the investigation. The total intensity difference between control and reference was $5.5 \%$ after $20 \mathrm{~min}$. This translates to only a $30 \%$ lower end state of the control compared to the reference. Also, as indicated by the error bars, an overlap between control and the other experiment is quite possible, making it difficult to draw conclusions. 
The $\mathrm{P} / \mathrm{L}=1: 500$ measurements show an overall lower total intensity increase. Nonetheless, the difference between control and the reference measurement in the total signal intensity is $8.2 \%$, making the control measurement $65 \%$ less efficient than the reference. Furthermore, all $\mathrm{P} / \mathrm{L}=1: 500$ measurements showed less deviation in the individual experiments, hence more consistent data was produced.

Another improvement was observed in the vesicle stability. For this experiment, vesicles with the purified peptides incorporated were prepared and immediately analyzed via DLS. Afterwards, the stock solution of the vesicles was left at room temperature for 15 days and subsequently analyzed again. The experiments were performed with both P/L ratios as illustrated in Figure 4.6.

(a)

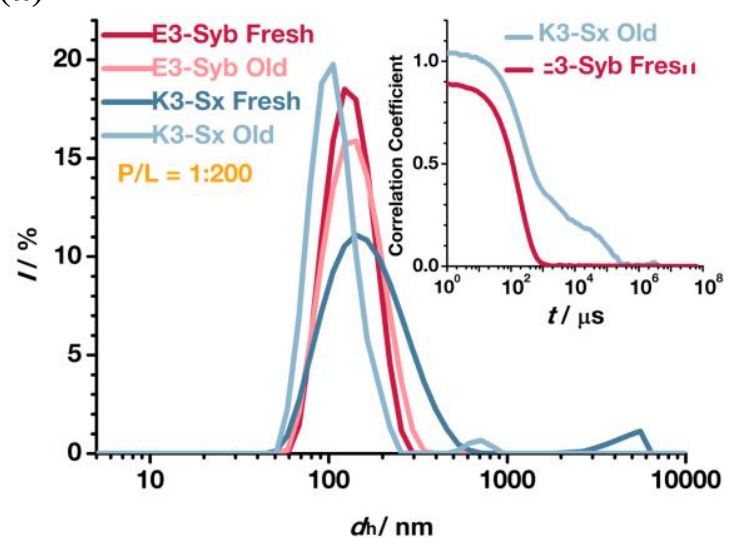

\begin{tabular}{lcc} 
Peptide & $\begin{array}{c}\text { Z-average } \\
\boldsymbol{d}_{\mathbf{h}} / \mathbf{n m}\end{array}$ & PdI \\
\hline E3-Syb Fresh & 125 & 0.069 \\
E3-Syb Old & 129 & 0.103 \\
K3-Sx Fresh & 154 & 0.254 \\
K3-Sx Old & 380 & 0.450 \\
\hline
\end{tabular}

(b)

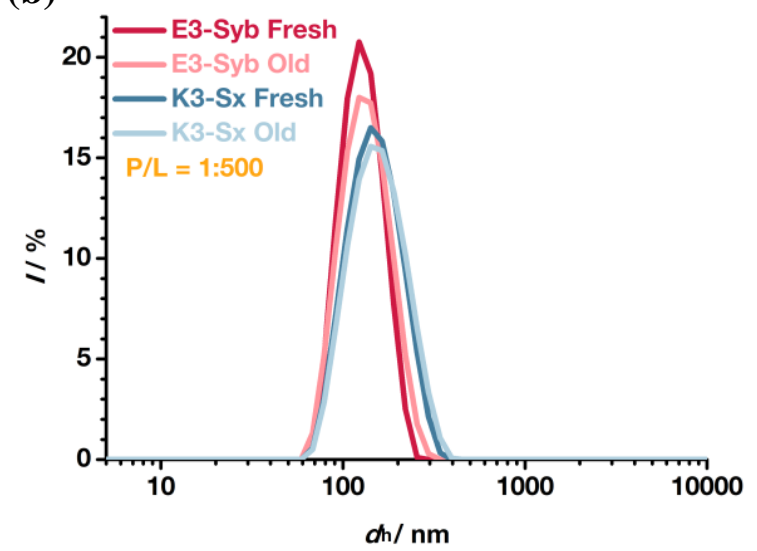

\begin{tabular}{lcc} 
Peptide & $\begin{array}{c}\text { Z-average } \\
\boldsymbol{d}_{\mathbf{h}} \text { /nm }\end{array}$ & PdI \\
\hline E3-Syb Fresh & 122 & 0.047 \\
E3-Syb Old & 126 & 0.071 \\
K3-Sx Fresh & 140 & 0.094 \\
K3-Sx Old & 145 & 0.111 \\
\hline
\end{tabular}

Figure 4.6: DLS data comparison of vesicle species with different P/L ratios and different age. (red) E3-Syb vesicles. (blue) K3-Sx vesicles. Dark colors depict vesicles which were measured within 5 min after extrusion (fresh). Pale curves show measurements after 15 days (old). (a) Measurements with $P / L=1: 200$. The correlation data of the old K3-Sx and fresh E3-Syb measurement is shown for comparison. (b) Measurements with $\mathrm{P} / \mathrm{L}=1: 500$ vesicles.

E3-Syb vesicles did not show a significant change regarding the DLS signals after 15 days regardless of which peptide to lipid ratio was used. Surprisingly, the same effect was observed with $\mathrm{K} 3-\mathrm{Sx}$ vesicles with $\mathrm{P} / \mathrm{L}=1: 500$. Both, the change of the mean diameter and the PdI was minimal and no visible precipitate could be seen. On the other hand, K3-Sx 
species with $\mathrm{P} / \mathrm{L}=1: 200$ showed significant formation of precipitate as well as a noticeable shift to bigger vesicle sizes. Unfortunately, the size representation of the old K3-Sx sample is not accurate due to the macroscopic particles in the sample. The correlation data shown in Figure 4.6, illustrates, that the 15 days old K3-Sx vesicles cannot be described sufficiently with the mean diameter and PdI. This is also true for the fresh K3-Sx sample due to the polymodal character of the sample.

In conclusion, changing the P/L to 1:500 improved the stability and size distribution of the K3-Sx vesicles significantly. In addition, the deviations of the individual FRET dequenching assays were significantly lowered, improving the overall data quality in respect to reliability, reproducibility and handling of the sample.

\subsubsection{Effect of Fluorophores}

The FRET pair used for the fusion assays are Lissamine rhodamine B (Rh) and N-(7-nitro2-1,3-benzoxadiazol-4-yl) (NBD) which are attached to the lipid anchor DOPE. The fluorophores are located at the polar head group region and can therefore interact with peptides or proteins. ${ }^{[231,232]}$ Depending on the $\mathrm{pH}$, the fluorophores are likely charged and can therefore interact with other charged molecules. To investigate whether this is also the case for the E3/K3-TMD model system, experiments involving the fluorophores monitoring the effect on fusion assays and vesicle sizes have been performed. The impact of the interaction between fluorophore and E3/K3-TMD peptides can be seen in Figure 4.7. In these experiments there are two main differences between the curves apparent. First, they differ in the matter of which vesicle population the FRET fluorophores are incorporated. Second, the ratio at which the vesicle species are mixed stays the same regarding the labeled and unlabeled species. This means, that the experiments from the red curve have a ratio of E3-Syb vesicle/K3-Sx vesicle = 1:4 and the blue curve the inverted ratio. From a statistical standpoint it does not matter which population is more abundant. However, this might still affect the fusion assays in an unexpected manner.

Primarily, FRET dequenching assays were performed with the fluorophores present in the E3-Syb vesicles (red curve). In this case an increase in signal intensity and an expected curve form can be observed in the fusion assay. On the other hand, incorporation of the fluorophores in the K3-Sx vesicles lead to a very small intensity increase, comparable with control measurements. 


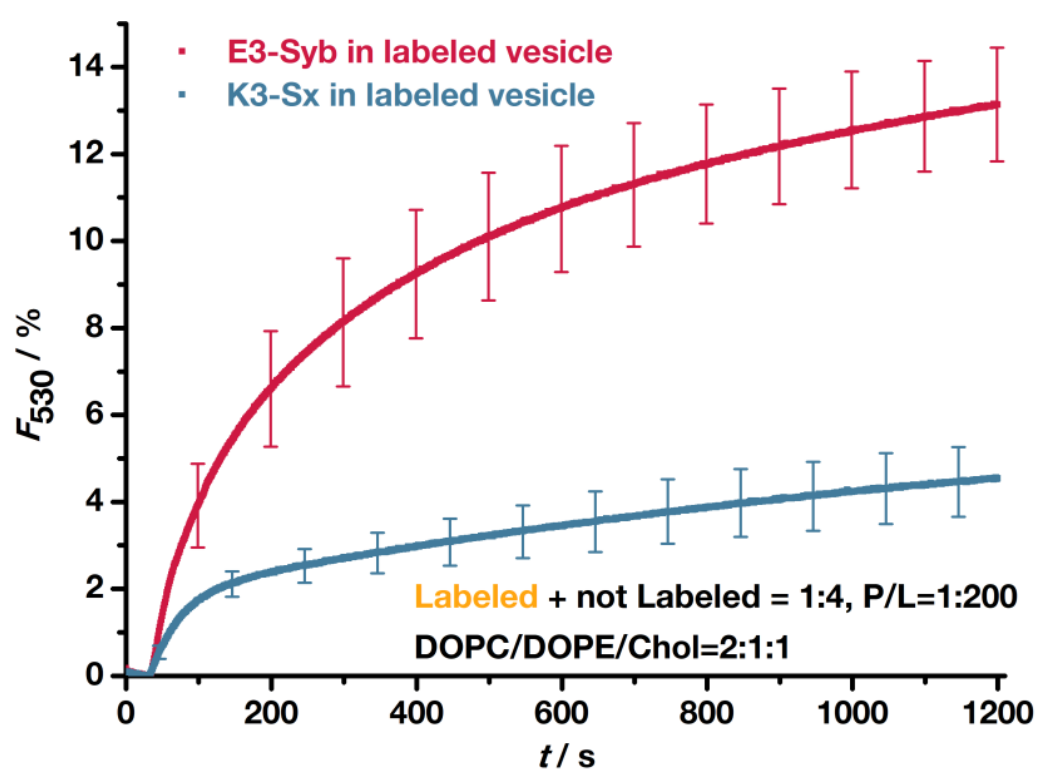

Figure 4.7: FRET dequenching assays of different labeled vesicle species. (red) Standard assay with E3-Syb peptides in the labeled vesicle. (blue) K3-Sx peptide in the labeled vesicle. The labeled vesicles contained NBD-DOPE and Rh-DOPE lipids, each at $1.5 \mathrm{~mol} \%$. The measurements were performed with $\mathrm{P} / \mathrm{L}=1: 200$.

Apparently, the location of the fluorophores matters in this assay with the E3/K3-TMD models system. There are three possible explanations for this behavior. First, K3-Sx interacts with the fluorophores rather than or in addition with E3-Syb to perform fusion. This hypothesis would explain the high signal increase in the standard fusion assay with the labels incorporated in the opposing vesicles of K3-Sx. This can be disproven by doing the same experiment but without the incorporation of the E3-Syb peptide. The experiment is discussed in more detail in section 4.3.4. Second, K3-Sx sticks to the own membrane more strongly due to the fluorophores interacting electrostatically with it. In this case fusion efficiency would suffer as the peptide to peptide interaction would decrease. This would explain the blue curve in Figure 4.7. Third, the ratio of E3-Syb vesicles and K3-Sx vesicles in the sample has a direct impact on the fusion efficiency.

To elaborate on the possible explanations, DLS experiments were performed concomitant to the fusion assays (see Table 4.2). Comparison of the E3-Syb vesicle sizes shows a small difference $(\sim 5 \mathrm{~nm})$ depending on the presence of fluorophores in the same vesicle. The size difference of the K3-Sx vesicles is noticeably bigger with $\sim 20 \mathrm{~nm}$ indicating an effect of the fluorophores. Furthermore, the comparison between the different vesicle species is also showing differences regarding the fluorophore placement. Generally, K3-Sx vesicles are 10-15 nm bigger in diameter compared to E3-Syb vesicles. Especially using the reference peptides $\mathrm{K} 3-\mathrm{Sx}(\mathrm{QSK})$ and $\mathrm{E} 3-\mathrm{Syb}(\mathrm{RKY})$, the sizes were fairly consistent and were 
therefore used as a vesicle quality indicator. In the case of the experiments with labeled K3-Sx vesicles, both vesicle species show a nearly identical mean size average of $144 \mathrm{~nm}$. This result is unexpected and differs from other experiments. A possible explanation for this behavior is that the fluorophores interact with $\mathrm{K} 3-\mathrm{Sx}$ in such a way that it is not capable of interacting with other vesicles. As mentioned in chapter 2.7.3, the K3-motif of the similar E3/K3-PEG-lipid model system was proposed to attach to the membrane, disturbe it and consequently facilitate fusion. ${ }^{[197,199,202,205]}$ Though, this effect was most efficient using $\mathrm{PEG}_{12}$ which has a length of $4.4 \mathrm{~nm}$ and is therefore significantly longer than the linker region of syntaxin. ${ }^{[197]}$ Another hint for the K3-membrane interaction comes from the vesicle sizes of the mixed population.

Table 4.2: Sizes of differently labeled vesicle species obtained via DLS. The sizes differ depending on the presence of fluorophores in the same membrane. Vesicles were prepared with $\mathrm{P} / \mathrm{L}=1: 200$.

\begin{tabular}{ccccc} 
Vesicle & $\begin{array}{c}\text { E3-Syb vesicle labeled } \\
\text { Z-average } \\
\boldsymbol{d} \text { /nm }\end{array}$ & PdI & $\begin{array}{c}\text { K3-Sx vesicle labeled } \\
\text { Z-average } \\
\boldsymbol{d h} / \mathbf{n m}\end{array}$ & PdI \\
\hline E3-Syb & 139.8 & 0.099 & 144.4 & 0.106 \\
K3-Sx & 163.1 & 0.192 & 143.3 & 0.098 \\
Mix & 200.6 & 0.219 & 156.7 & 0.131 \\
\hline
\end{tabular}

In the standard fusion arrangement (E3-Syb vesicles labeled) a compelling size increase was observed after mixing. In combination with the results of the dequenching assay, fusion of the two vesicle species is most probable. In contrast, the inverse fusion arrangement (K3-Sx vesicles labeled) does only show a moderate size increase, which supports the corresponding fusion assay results.

It has to be noted, that these experiments were performed with $\mathrm{P} / \mathrm{L}=1: 200$. Therefore, the sizes and the polydispersity index for the K3-Sx vesicles are larger than usual. A straight up comparison to $\mathrm{P} / \mathrm{L}=1: 500$ measurements has to be made cautiously. However, the before mentioned effects are more pronounced here than with a lower peptide to lipid ratio and accordingly emphasize the obtained observations.

In summary, placement of the fluorophores for FRET dequenching assays appears to matter in the case of the E3/K3-TMD model system. Especially the vastly different sizes of the K3-Sx vesicles indicate an involvement of the fluorophores in the vesicle behavior. Still, the possibility of an effect due to the ratio of the vesicles in the mixed sample has not been ruled out and may thus be also a contributor to these observations. 


\subsubsection{Assessment of Control Experiments}

Control experiments play an important role for the interpretation of data. Effects which are not due to the intended event but rather to other phenomena, can be quantified and accounted for. Especially in the employed FRET dequenching assays with different vesicle and peptide species, many factors have to be considered during data interpretation. Thus, several different controls have been tested to identify the best experiment setup. Figure 4.8 depicts three different controls which were tested.

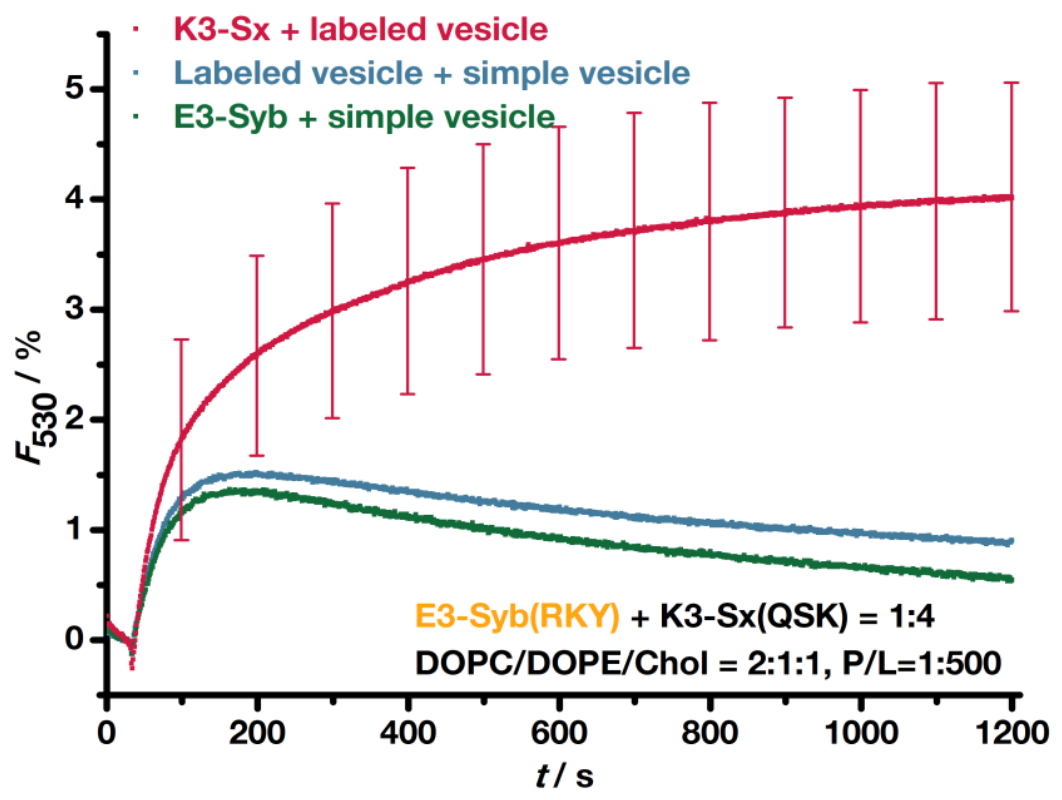

Figure 4.8: Comparison of FRET dequenching assay control measurements using different vesicle species. (red) K3-Sx vesicles interacting with labeled vesicles. (blue) Labeled vesicles interacting with simple vesicles. (green) Labeled E3-Syb vesicles interacting with simple vesicles. Simple vesicle refers to the standard lipid composition of DOPC/DOPE/Chol = 2:1:1 without peptides. All measurements including peptides were done with a $\mathrm{P} / \mathrm{L}$ ratio of 1:500 with the reference peptides K3-Sx(QSK) and E3-Syb(RKY).

The simplest control consists of two vesicles containing no peptide. The labeled vesicles contained the FRET fluorophores, whereas the simple vesicles consisted only of DOPC, DOPE and cholesterol. Upon mixing the two vesicle species (at $30 \mathrm{~s}$ ), a minimal increase of fluorescence intensity is observed. The maximum is reached $150 \mathrm{~s}$ after mixing. This small artefact is detected for every labeled vesicle type and is most likely an effect due to the polarity change around the embedded NBD. ${ }^{[233]}$ The addition of the second vesicle species decreases the polarity and increases the fluorescence intensity. The same curve shape was observed in the experiment with labeled E3-Syb and simple vesicles (green 
curve). The fusion assay results of these two experiments suggest that no lipid mixing of the opposing membranes in any way occurred. Conversely, the results of the unlabeled $\mathrm{K} 3-\mathrm{Sx}$ vesicles interacting with the labeled vesicles show a clear increase in signal intensity after the mixing point at $30 \mathrm{~s}$. The shape of the curve resembles a typical fusion curve of this model system. Additionally, the fluorescence intensity is much higher compared to the other two control experiments. This general trend was observed for every type of K3-Sx except K3-Sx(QSXARRXK). Apparently, the presence of the K3-Sx peptide triggers a positive signal in the experiment. To investigate whether K3-Sx by itself is sufficient to initiate the fusion process, DLS measurements were performed (Table 4.3).

Table 4.3: Vesicle sizes of liposomes from varying control experiments obtained via DLS. Mixed populations consist of a ratio of labeled/unlabeled $=1: 4$ vesicles after an incubation time of at least $20 \mathrm{~min}$.

\begin{tabular}{cccc} 
Experiment & Vesicle & $\begin{array}{c}\text { Z-average } \\
\boldsymbol{d} \mathbf{h} / \mathbf{n m}\end{array}$ & PdI \\
\hline \multirow{2}{*}{ K3-Sx + labeled } & K3-Sx & 141.2 & 0.095 \\
& Labeled & 117.8 & 0.066 \\
& Mix & 142.1 & 0.138 \\
\hline \multirow{2}{*}{ Labeled + simple } & Simple & 124.1 & 0.044 \\
& Labeled & 120.6 & 0.068 \\
\hline \multirow{2}{*}{ E3-Syb labeled +} & Mix & 125.0 & 0.074 \\
simple & Simple & 122.4 & 0.059 \\
& E-Syb labeled & 124.5 & 0.054 \\
\hline
\end{tabular}

For the fusion experiment of K3-Sx and a labeled vesicle the DLS data is not conclusive. The size of the mixed population is nearly identical with the size of the K3-Sx vesicle. Though, the polydispersity index is considerably higher which is to be expected as a mixture of at least two different vesicle types with a big size difference $(\sim 20 \mathrm{~nm})$ are present in the measured sample. This observation was also discovered for the other two controls. Due to the ratio of labeled/unlabeled vesicle of 1:4 in the mixed sample, it is difficult to draw conclusions of the DLS results in these control measurements. A small increase in the fusion assay suggests only a small number of vesicles fusing. In the case of DLS measurements, the intensity increase is not sufficient to see a noticeable average size increase. Consequently, it cannot be ruled out that $\mathrm{K} 3-\mathrm{Sx}$ is capable of performing fusion on its own to a small extent. However, it is also possible that hemifusion, other unspecific interactions or the destruction of the labeled vesicles are the reason for the obtained FRET 
data. Undeniably the peptide is interacting in some way with the labeled vesicle due to the completely different curve form in the fusion assay. A possible explanation for the obtained data is the interaction of the K3-motif with the labeled vesicle. Here, the "snorkeling" effect of lysins as well as the interaction of the hydrophobic face with the membrane can be part of this strong interaction. ${ }^{[197,199,202,205]}$ As the motif contains six lysines, this effect is likely to happen. In fact, researchers using a similar model system with the E3/K3 motif showed this behavior to be critical for high fusion efficiency (see chapter 2.7.3). ${ }^{[197]}$ In addition, due to the fluorophores the labeled vesicle contains negatively charged lipids. ${ }^{[231,232,234]}$ It is plausible, that the positively charged $\mathrm{K} 3$ motif interacts more easily with the labeled vesicle, leading to the obtained results.

To summarize, E3-Syb peptides by themselves do not show signs of being capable to perform fusion or destroy the membrane they are incorporated in. For K3-Sx vesicles a different behavior was observed. Although, it is not clear what the underlying reason of the intensity increase is, $\mathrm{K} 3-\mathrm{Sx}$ seems to be responsible for it. To draw a conclusion, the control including K3-Sx and a labeled vesicle is the most reasonable, if a K3-Sx peptide is involved in the experiment. The inherent trait of this peptide to give a positive signal in the FRET dequenching assay has to be taken into account when interpreting the fusion assays. Nevertheless, compared to experiments with both peptides present, a clear distinction between control and fusion experiment is possible. Furthermore, the change to a peptide to lipid ratio of 1:500 overall decreased the effect of the K3-Sx peptide on the fusion assay as illustrated in Figure 4.5. Therefore, the specific interaction between the different peptides can still be considered the main reason for positive fusion signals.

\subsubsection{Loss of Lipids during Extrusion}

The following experiments were performed mostly by Fehmke Reichardt during her bachelor thesis under the supervision of Mike C. Groth. ${ }^{[235]}$

Aggregation of liposomes was a significant problem in previous experiments which lead to a loss of lipid and peptide material during and after liposome preparation. ${ }^{[207]}$ Two main factors for the loss were already identified. First, during the extrusion process, where MLVs are pushed through a polycarbonate membrane, a lot of material adheres to it. This can be seen clearly in case of labeled liposomes, as the polycarbonate membrane changes its color 
from white to pink. Second, self-aggregation of liposomes of the same species over time which concludes in the formation of precipitate.

Determination of lipid loss before and after extrusion was performed for selected vesicle species via phosphate test. For experimental details see section 9.4.6. The results are presented in Table 4.4.

Table 4.4: Results of phosphate tests for different vesicle species. Lipid loss is obtained by the calculated input of phospholipids.

\begin{tabular}{lcc} 
Peptide in vesicle & No. & Lipid loss / \% \\
\hline E3-Syb(RKY) & (1) & $34 \pm 7$ \\
E3-Syb(RKYAW) & (3) & $32 \pm 2$ \\
E3-Syb(RKYWA) & (4) & $31 \pm 9$ \\
K3-Sx(QSK) & (2) & $42 \pm 10$ \\
K3-Sx(QSXARRXK) & (5) & $49 \pm 7$ \\
\hline
\end{tabular}

Overall, lipid loss lies in between 30-50 \% with a high dependency on the incorporated peptide. Lipid films with K3-Sx peptides tend to be affected with 10-15\% more lipid loss than E3-Syb containing films. This result is in agreement with previous experiences and observations from other experiments.

A drawback of this method is that the amount of peptide loss cannot be determined. Merely the number of phospholipids (eg. DOPC, DOPE, DOPS) can be obtained. As cholesterol does not feature a phosphate group, it also stays invisible for this method. With the peptide to lipid ratio a rough estimation of the number of peptides left in the sample could be calculated. But this approach does not take into account, that the peptides might interact with each other, cholesterol or the polycarbonate membrane, forming aggregates which stick to the membrane or are too big to pass it. Due to these many compromises and inaccuracies, this method was used only for few measurements. 


\section{Modifications of the E3/K3-TMD Model System}

The focus of this chapter lies on the E3/K3-TMD model systems response to modifications in the peptide sequences during fusion assays. In section 5.1 the effect of the linker length on fusion efficiency is discussed. Here, the specific connection between artificial and the natural part of the system and the resulting structural conformation is investigated. Sections 5.2 and 5.3 portray the importance of the aromatic amino acids, which are present in both linkers. Some of these residues have been found to be important during the fusion mechanism of natural SNARE complexes. The effects on this model system are examined.

\subsection{Effect of the Linker Length on Fusion Efficiency}

The length of the linkers in Synaptobrevin and Syntaxin have been of interest for a long time. As the stiffness or flexibility is postulated to be a key feature of the linker, several experiments have been performed regarding its length in past years. ${ }^{[25,170,236,237]}$ In fact, one experiment focusing on linker length with the E3/K3-TMD model system has already been performed. ${ }^{[206]} \mathrm{K} 3-\mathrm{Sx}$ was elongated with its native sequence by six amino acids. In content mixing assays the elongated peptide performed slightly less efficient compared to the reference model system. To further understand the linkers function in the model system, multiple experiments are presented in the following section regarding the linker length. 


\subsubsection{Sequence Shift}

The reference system which consists of the two peptides E3-Syb(RKY) and K3-Sx(QSK) was designed in such a way, that the sequence length of the natural TMD and linker is equally long. For both species 30 amino acids starting from the C-terminus were counted and cut off from the rest of the natural protein. Amino acid 31 is part of the artificial E3/K3 coiled coil (see Figure 5.1).

(a)

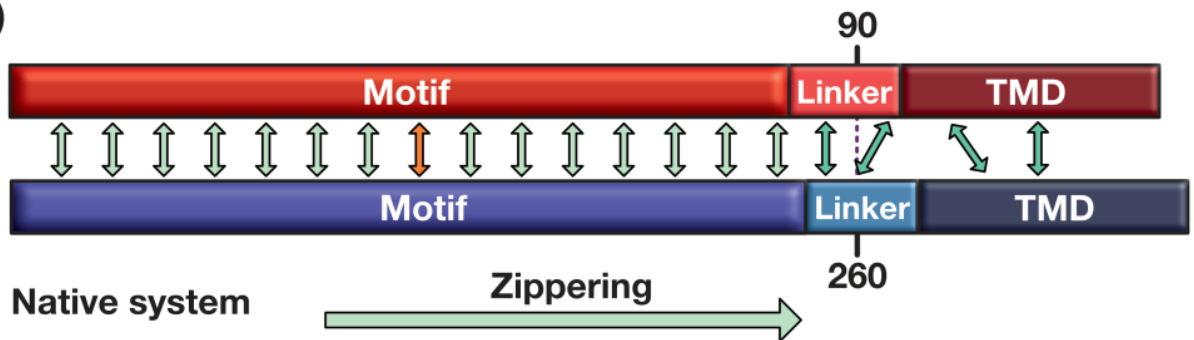

(b)

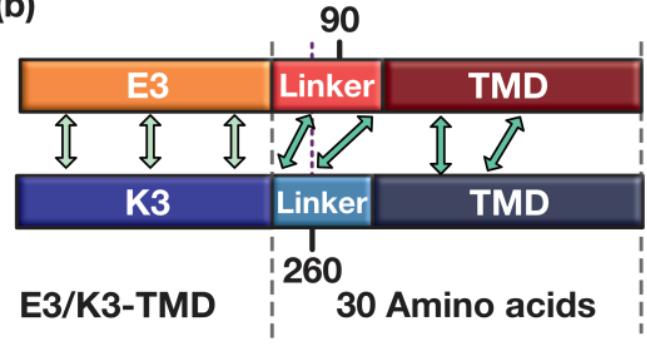

(c)

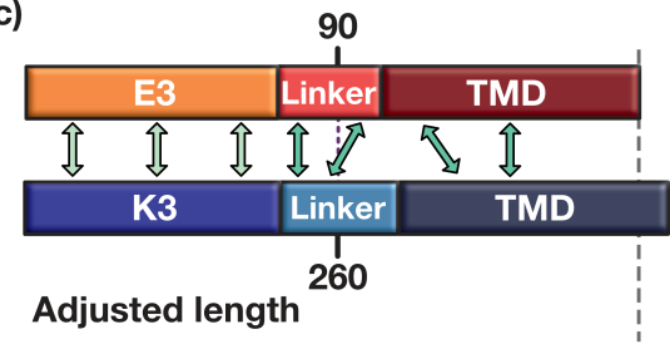

Figure 5.1: Visualization of the shift in the sequence between natural protein and model system. The arrows indicate possible interactions between the two proteins/peptides and visualize the shift due to the attachment of the artificial motif after 30 amino acids. (a) Native sequence, (b) E3/K3-TMD standard sequence, (c) sequence with adjusted length.

In the assembled natural SNARE complex, the amino acids 90 of synaptobrevin and 260 of syntaxin are aligned with each other. Specific interactions between different residues in the linker and TMD have been proposed previously and are illustrated with double sided arrows in Figure 5.1. ${ }^{[23]}$ Interaction between the two model system peptides starts at the $\mathrm{N}$-termini with the formation of the coiled coil. The alignment starts here and propagates toward the C-termini. Assuming the conformation of the linker and TMD of the artificial system stays the same as in the natural complex, a shift of two amino acids is expected starting with the first amino acid of the linker in the model system. This is due to the fact that in the natural complex, syntaxin has an overhang of two amino acids at the C-terminus. ${ }^{[23]}$ To elucidate the significance of the right sequence position in the 
E3/K3-TMD model system, the peptides have been adjusted in length to depict the situation in the natural one (Table 5.1).

Table 5.1: Comparison of the peptide sequences for the experiments targeting sequence shift. The E3/K3 motif is omitted. Amino acids of the linker domain are denoted in bold.

Peptide

Sequence

No.

Sx(QSK) QS KA R RKK I M I I I C C V I L G I I I A S T I GG I F G

Sx(KYQSK) KY Q S K A RRKK I M I I I C C V I L G I I I A S T I GG I F G (6)

Syb(RKY) R K Y WWK N L KMM I I L G V I C A I I L I I I I V Y F S T

The peptides K3-Sx(QSK) and E3-Syb(RKY) are referred to as the standard system in this thesis. Both feature 30 amino acids from the natural SNARE proteins. The sequence shift adjusted peptide K3-Sx(KYQSK) has 32 amino acids from the natural sequence. On paper, if the assembly of the heterodimeric complex starts from the $\mathrm{N}$-terminus and continues throughout the TMD, then the positions of the involved amino acids should resemble the natural complex as depicted in the X-ray data of the cis-complex. ${ }^{[23]}$ The results of the FRET dequenching assay are illustrated in Figure 5.2.

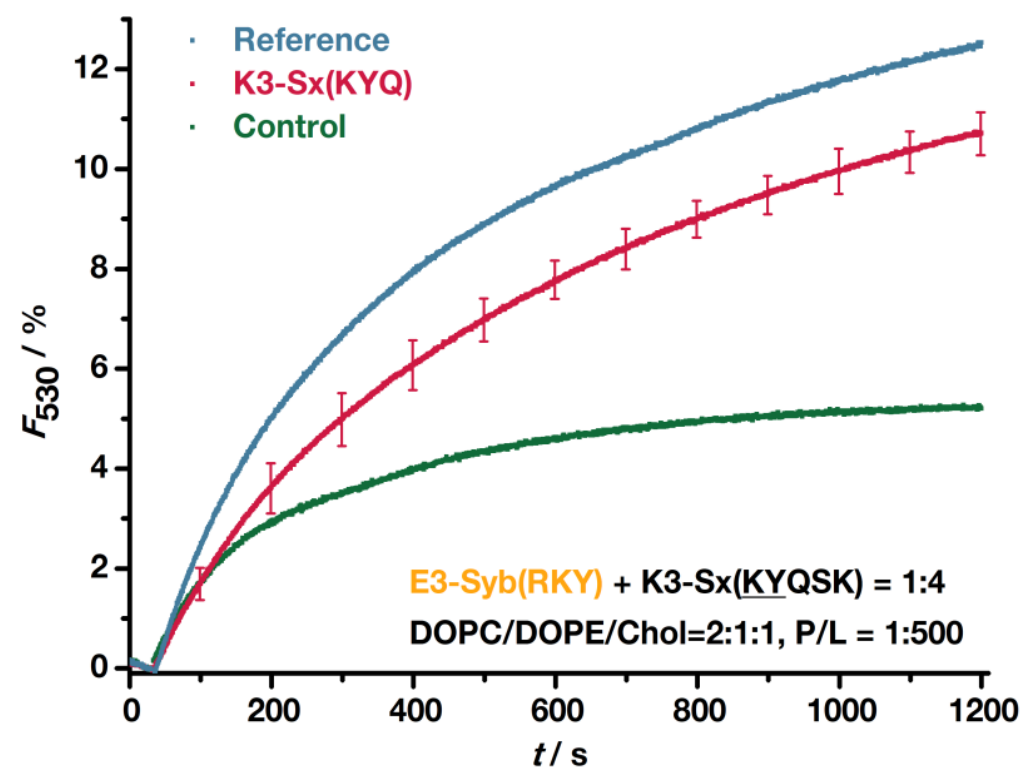

Figure 5.2: FRET dequenching assay of E3-Syb(RKY) and K3-Sx(KYQSK). The measurements (red), standard system as reference (blue) and a control measurement (green) consisting of labeled vesicles without peptide and K3-Sx(KYQSK) vesicles are shown.

The size adjusted system shows an unambiguous positive signal. Compared to the reference, the fusion efficiency after $20 \mathrm{~min}$ is slightly lower. Also, the slope is less steep 
at every time point during the experiments. The error of the measurement is quite small, but this is due to the experiment being performed only two times. The control experiment shows a relatively high signal compared to other controls in this work. The acquired DLS data validates the results obtained from the fusion assays (Table 5.2).

Table 5.2: Vesicle sizes of liposomes from experiments targeting the linker length of the model system. The data was obtained via DLS.

\begin{tabular}{lccc} 
Measurement & Vesicle & $\begin{array}{c}\text { Z-average } \\
\boldsymbol{d}_{\mathbf{h}} / \mathbf{n m}\end{array}$ & PdI \\
\hline \multirow{2}{*}{ E3-Syb + K3-Sx } & E3-Syb(RKY) & 132.3 & 0.056 \\
& K3-Sx(KYQSK) & 144.5 & 0.112 \\
& Mix & 161.5 & 0.143 \\
Control & Labeled & 125.0 & 0.067 \\
& K3-Sx(KYQSK) & 144.2 & 0.124 \\
& Mix & 144.0 & 0.123 \\
\hline
\end{tabular}

The measurements with both peptides clearly show an increase in vesicle sizes after mixing, confirming the length adjusted systems fusion capabilities. The control measurement does not show a size change in the mixed population. Reasons for the mixed results of the dequenching assay and DLS data are discussed in section 4.3.4.

To summarize, the sequence adjusted peptide performs slightly less efficient than the reference system. The misalignment on paper does not correlate to the experimental results, suggesting the reference sequence to be aligned ideally for the E3/K3-TMD model systems fusion abilities.

\subsection{Aromatic Residues in the Linker Domains}

The X-ray structure of the natural SNARE complex of syntaxin 1A and synaptobrevin 2 shows multiple aromatic residues present in the linker. These are arranged in such a way, that they wrap around the synaptobrevin linker to form a collar of aromatic residues. It is assumed that these aromatic residues stiffen the linker region due to intermolecular interactions. ${ }^{[23]}$ Furthermore, the tryptophan residues of synaptobrevin are proposed to be controlling the positions of adjacent lysine and arginine residues. ${ }^{[29]}$ The following chapter is about the significance of the mentioned amino acids for the functionality of the E3/K3-TMD system. 


\subsubsection{E3-Syb Tryptophans}

Synaptobrevin has been of interest for researchers for many decades. Especially, the two tryptophans in the linker have been studied by several research groups. ${ }^{[237,238]}$ In chromaffin cells, different experiments including substitutions of these amino acids have been performed. Interestingly, the substitution of just one of the tryptophans with serine or alanine did not affect the exocytotic burst of chromaffin cells. ${ }^{[29]}$ Nonetheless, it was also suggested, that the tryptophans act as a fusion clamp making fusion stimulus-dependent. ${ }^{[30]}$ Furthermore, MD simulations lead to the conclusion, that the tryptophan residues determine the insertion depth of the protein in the membrane. ${ }^{[29]}$ Due to the adjacent basic residues, this has an effect on the electrostatic potential on the fusion site. The tryptophan motif was also linked to the rigidity of the linker itself. ${ }^{[129]}$ A substitution with alanine lead to a more flexible linker, highlighting the importance of these residues. The position of the residues as well as the approximate insertion in the membrane are illustrated in Figure 5.3.

(a)

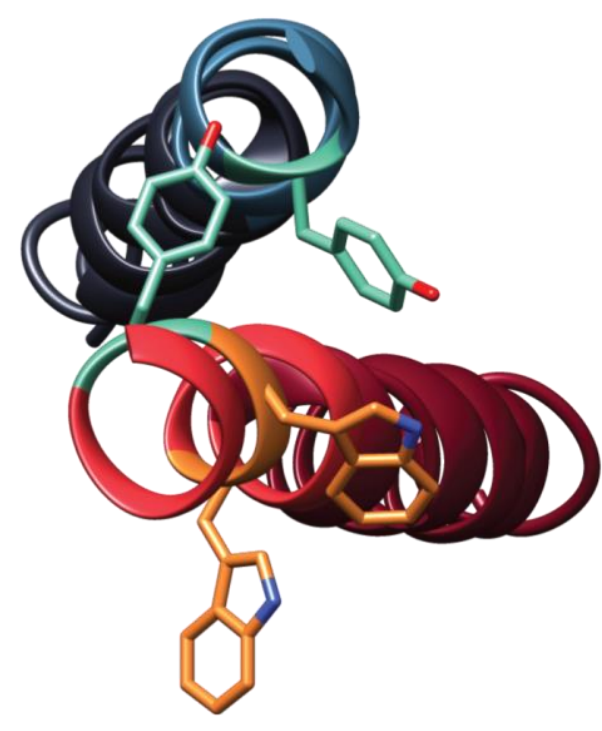

(b)

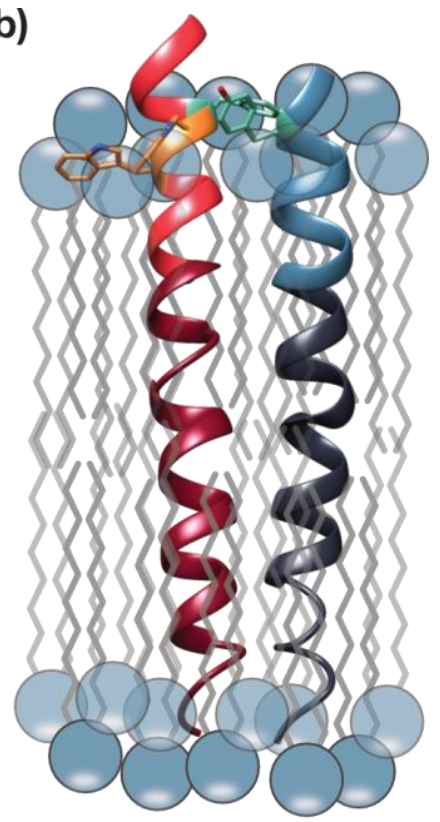

Figure 5.3: Illustration of the aromatic amino acids in the linkers of synaptobrevin 2 and syntaxin 1A. Tyrosins are colored in teal, tryptophans are colored in orange, syntaxin $1 \mathrm{~A}$ is colored blue and synaptobrevin 2 is red. (a) Interface of both proteins from X-ray data. (b) Illustration of the SNARE complex inside a membrane. X-ray data from STEIN et al. ${ }^{[23]}$ Molecular graphics and analyses were performed with the UCSF Chimera package. ${ }^{[121]}$

Investigating these aromatic amino acids with the less complex E3/K3-TMD model system might yield further information regarding their tasks in membrane fusion. With this system, 
interactions with associated proteins can be excluded and parts of their functions isolated. This approach is also working vice versa, as the results can be compared to the studies with the associated proteins and therefore make predictions about their importance in this process. However, as the E3/K3 motif behaves differently to the natural motif, conclusions must be drawn conservatively.

To determine the effects of WW 89/90 of synaptobrevin 2 in the E3/K3-TMD model system, three peptides have been synthesized (Table 5.3). Peptide $\mathbf{3}$ and $\mathbf{4}$ have only one substitution, whereas peptide 7 has both tryptophans substituted with alanine. The substitution was performed using alanine for its low interaction with other residues, neutral net charge and helix favoring traits. Furthermore, the results can be compared more easily with other systems including the natural complex.

Table 5.3: Peptide names and sequences synthesized for the determination of the role of the WW-domain in synaptobrevin 2.

\begin{tabular}{llc} 
Peptide & Sequence & No. \\
\hline E3-Syb(RKYAW) & G-(EIAALEK) 3 -RKYAWKNLK-TMD & (3) \\
E3-Syb(RKYWA) & G-(EIAALEK) 3 -RKYWAKNLK-TMD & (4) \\
E3-Syb(RKYAA) & WG-(EIAALEK) 3 -RKYAAKNLK-TMD & $\mathbf{( 7 )}$ \\
\hline
\end{tabular}

Synthesis and purification of these peptides did not show significant changes in behavior compared to the standard E3-Syb peptide (see sections 9.2.7 and 9.3). The peptide E3-Syb(RKYAA) had to be modified with an additional tryptophan residue at the $\mathrm{N}$-terminus. This is due to the light absorption properties at $280 \mathrm{~nm}$ which were used for the determination of the peptides concentration (see chapter 9.2.6). Indeed, tyrosine is also absorbing at this wavelength, but at a much lower efficiency. Consequently, much more sample material would be needed for the determination of the peptide concentration. As the final amount of purified peptides is quite small, the addition of an N-terminal tryptophan was reasonable. Also, the $\mathrm{N}$-terminal ends of the E3/K3 motif should not interact strongly with each other.

The results of the FRET dequenching assays show a slight reduction in fusion efficiency for all three peptides compared to the reference (Figure 5.4). It is quite remarkable, that the substitution of one amino acid has such a big impact on the efficiency of the system. This highlights the importance of the specific sequence of amino acids in the linker of synaptobrevin 2 . 
E3-Syb(RKYWA) shows an unusually small error margin due to two of the four measurements being nearly identical in their shape and intensity. This observation is most likely by chance, as no other experiment yielded such a small error. The error of the E3-Syb(RKYAW) measurement is substantial, whereas the double substituted peptide has a medium error. In general, all three peptides behave fairly similar in regard to curve form and end intensity. Due to their proximity and the associated error, conclusions can only be drawn conservatively in regard to the position of each other.

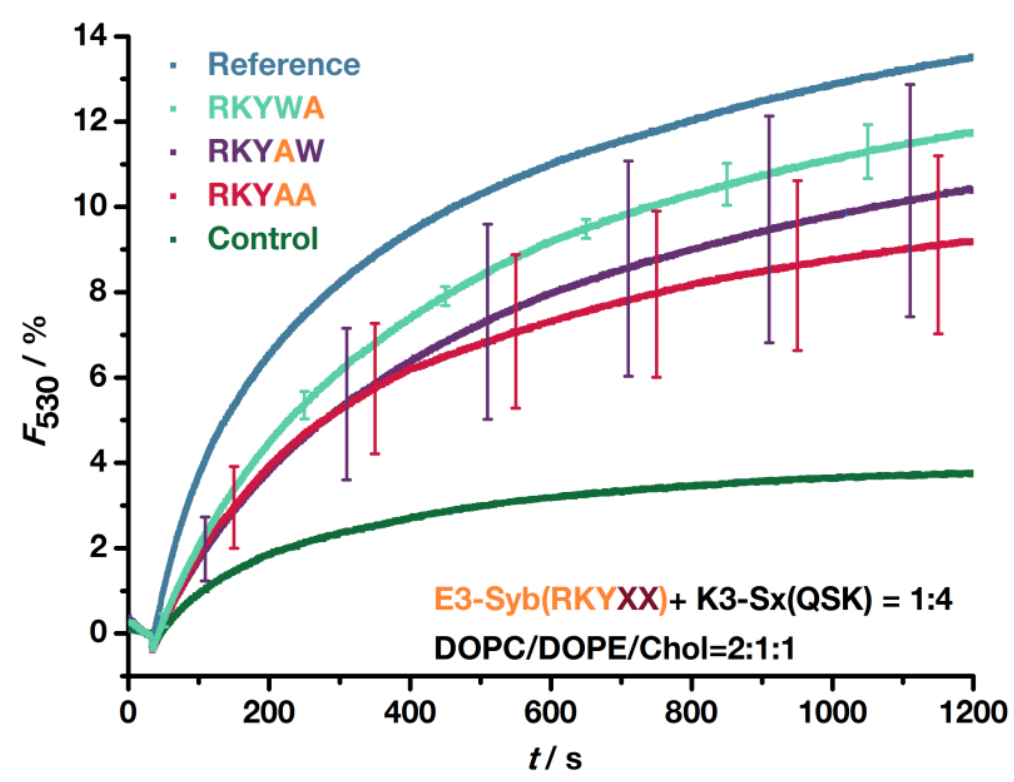

Figure 5.4: Comparison of different E3-Syb modified peptides. (blue) Reference measurement with the standard peptides. (teal) E3-Syb peptide with the second tryptophan substituted for alanine. (purple) E3-Syb peptide with the first tryptophan substituted for alanine. (red) E3-Syb peptide with both tryptophans substituted for alanine. (green) Control measurement. All measurements were performed at least thrice and with $\mathrm{P} / \mathrm{L}=1: 500$.

Apparently, it does not matter which of the tryptohans is substituted, as the curves are alike. Interestingly, the substitution of both tryptophans did not lower the fusion efficiency noticeably lower compared to single substitution. The lack of one residue is sufficient to interfere with the fusion process. Here, the difference of the model system with the natural SNARE complex becomes apparent. As initially mentioned, a study using chromaffin cells to investigate the WW-domain published the opposite result. ${ }^{[29]}$ Substitution of both tryptophans lead to a decrease in primed release-ready vesicles but did not change the kinetics of the process. One explanation for this difference might be, that the lack of just one tryptophan can be compensated by other proteins involved in the process. Another reason might be that the model system and the natural complex perform the fusion very 
differently in regard to the mechanism. In fact, the observations done by BORISOVSKA et al. referred to the priming process in which other proteins such as synaptotagmin or munc13/munc18-1 are involved. ${ }^{[94,239]}$ Due to other proteins interacting with the core complex, the process is more sophisticated compared to the E3/K3-TMD model system. Investigations about possible interactions between other proteins and the model system were not performed in this work.

The sizes of the used vesicles determined by DLS show homogenic distribution for all vesicle species (Table 5.4). All K3-Sx-vesicles, which used the same peptide, show an average size of $140 \mathrm{~nm}$. The PdI is well below 0.1 indicating a narrow monomodal distribution. Consequently, the vesicles were of proper quality.

Table 5.4: DLS data of the vesicles used for experiments regarding the WW-domain of synaptobrevins linker.

\begin{tabular}{lllll} 
Peptide & & \multicolumn{1}{c}{ E3-Syb } & K3-Sx(QSK) & \multicolumn{1}{c}{ Mix } \\
\hline \multirow{2}{*}{ E3-Syb(RKYAW) } & $d_{\mathrm{h}} / \mathrm{nm}$ & $124.9 \pm 3.8$ & $139.9 \pm 4.2$ & $152.1 \pm 1.4$ \\
& PdI & $0.062 \pm 0.023$ & $0.093 \pm 0.02$ & $0.124 \pm 0.05$ \\
\hline \multirow{2}{*}{ E3-Syb(RKYWA) } & $d_{\mathrm{h}} / \mathrm{nm}$ & $127.6 \pm 3.5$ & $140.1 \pm 3.9$ & $154.3 \pm 1.3$ \\
& $\mathrm{PdI}$ & $0.080 \pm 0.019$ & $0.105 \pm 0.021$ & $0.139 \pm 0.020$ \\
\hline \multirow{2}{*}{ E3-Syb(RKYAA) } & $d_{\mathrm{h}} / \mathrm{nm}$ & $128.3 \pm 2.1$ & $140.2 \pm 3.0$ & $147.0 \pm 1.9$ \\
& PdI & $0.084 \pm 0.036$ & $0.100 \pm 0.016$ & $0.126 \pm 0.018$ \\
\hline
\end{tabular}

The sizes of the E3-Syb vesicles did not differ in a significant amount. The reference vesicles size with the E3-Syb(RKY) peptide is around $125 \mathrm{~nm}$. The other E3-Syb species did not show noticeable changes in their sizes. The PdI for all species is also well below 0.1 , certifying their proper quality. Thus, the small sequence changes have no effect on vesicle sizes. This observation is an indication, that the peptides do not interact with the membrane in a significantly different manner compared to the reference peptide. DLS data of the mixed population also show an increase in particle size after $20 \mathrm{~min}$. In combination with the results of the FRET dequenching assay the three E3-Syb peptide variants seem to be able to perform vesicle fusion. Determination of lipid loss after extrusion also showed no significant difference compared to the reference, suggesting no major changes in vesicle precipitation due to the peptide (Table 4.4). Still, the changes in the amino acid sequence could influence the peptide's insertion depth or angle into the membrane. Clustering properties of the peptide could also be changed but not observed with the measurement techniques applied. Summarizing the results of the FRET dequenching assay and DLS 
measurements, an explanation for the reduced fusion efficiency could be linked to the insertion depth of the peptide inside the membrane. Recent MD simulations of the TMD with the linker showed, that the WW-domain is responsible for the insertion depth of the adjacent basic residues of the natural TMD of synaptobrevin. ${ }^{[29]}$ Nonetheless, a substantial change was only observed for the double substituted mutants. Again, the results obtained with the E3/K3-TMD system show an effect starting with the substitution of just one of the tryptophans. The reduced fusogenicity could also be an effect of a changed insertion angle into the membrane. The shallower the angle, the smaller is the distance of the motif from the membrane. This in return means, that the E3 motif is less able to reach for the K3 motif of the opposing vesicle. Reduced fusion activity could be the consequence.

To conclude, the WW-domain is important for the fusion behavior of the E3/K3-TMD model system. However, there are differences compared to the natural SNARE system. In contrast to nature, the model system shows a significant change upon the substitution of only one tryptophan. This suggests a different fusion mechanism. Due to the substantial changes in the structure as well as the absence of other proteins involved in the process, this result is not surprising. Besides, the model system is meant to reduce complexity and make small changes more noticeable. However, this makes it difficult to compare both the analog with the natural system.

\subsubsection{K3-Sx/E3-Syb Tyrosines}

The linkers of both peptides contain a total of four aromatic amino acids, two of which are tyrosines and the other tryptophan residues. One tyrosine is located in the linker of syntaxin, whereas the remaining three aromatic amino acids are located in synaptobrevin. Tyr 257 of syntaxin is buried inside a basic pocket of adjacent lysine residues (Figure 5.5). Previously, several substitution experiments have been performed with the focus of the linker, but only the substitution of Tyr 257 lead to a significantly reduced thermal stability. ${ }^{[23]}$ Furthermore, in the post-fusion complex, the tyrosine residues of both proteins are positioned facing each other. 
(a)

(b)

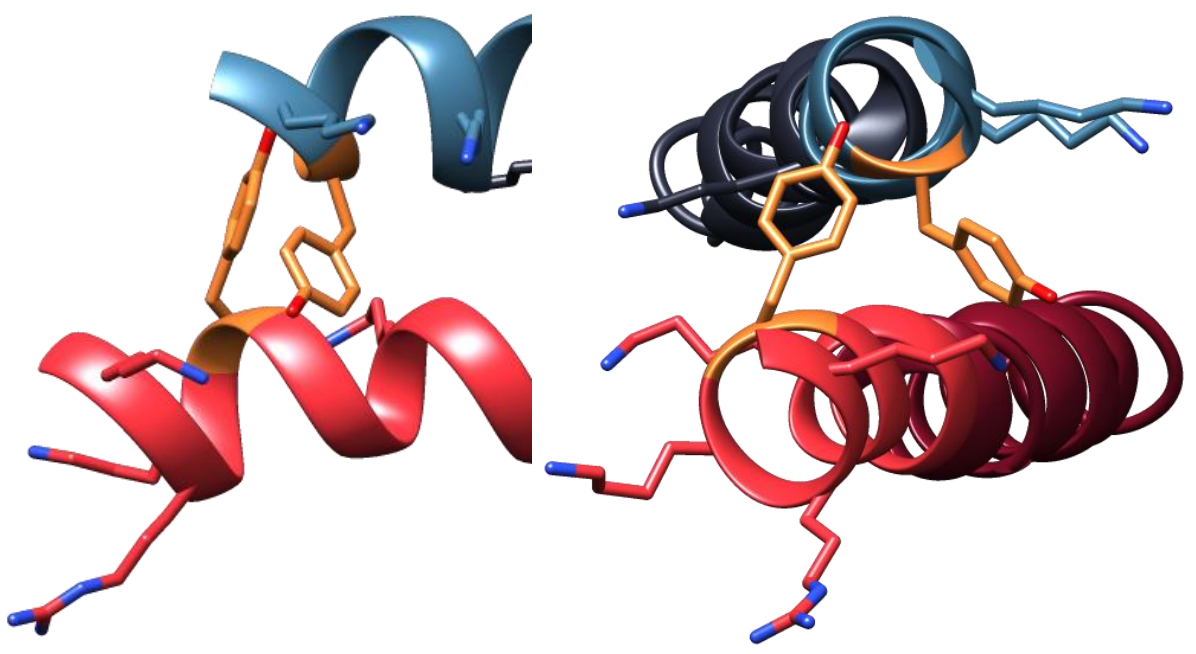

Figure 5.5: Linker and TMD of synaptobrevin 2 and syntaxin 1A in the post fusion cis configuration. Tyrosine residues which were substituted with alanine are marked in orange, synaptobrevin is red and syntaxin is blue. Basic residues surrounding the tyrosines are also displayed. (a) Side view. (b) Front view from $\mathrm{N}$ - to C-termini. X-ray data from STEIN et al. ${ }^{[23]}$ Molecular graphics and analyses were performed with the UCSF Chimera package. ${ }^{[121]}$

Due to their close proximity, interactions between those residues are probable. To investigate whether these two tyrosines are important for fusion in the model system, two new peptide species have been synthesized (Table 5.5). The E3-Syb peptide was altered by the substitution of Y88A referring to the position in the natural protein. The K3-Sx peptide had to be modified even more compared to the E3-Syb peptide. The sequence of the standard model system does not include the targeted tyrosine. Therefore, the linker had to be extended by at least one amino acid. In the end, two more residues from the native linker (KY) have been introduced to the new peptide. The additional lysine was added to maintain the basic environment around the tyrosine residues. Furthermore, the addition of two amino acids should change the relative positions of the linker in the complex (see section 5.1.1). Further insight into the importance of the right sequence shift could be gained with this peptide. Synthesis and purification were performed as described in the standard procedures without noticeable differences. 
Table 5.5: Peptide names and sequences with substituted tyrosine residues. Substituted amino acids are marked in orange. The K3-Sx peptide was elongated by two amino acids (underlined) as they are not present in the reference peptide.

\begin{tabular}{llc} 
Peptide & Sequence & No. \\
\hline E3-Syb(RKA) & G-(EIAALEK) 3 -RKAWWKNLK-TMD & (8) \\
K3-Sx(KAQSK) & WWG-(KIAALKE) $3-\underline{K}$ AQSKARRKK-TMD & (9) \\
\hline
\end{tabular}

The performed FRET dequenching assays show a high degree of variation regarding kinetics and total intensity increase (Figure 5.6). The average curve suggests a slightly less efficient fusion behavior compared to the reference system and a higher efficiency compared to the elongated reference. The latter one resembles the sequence of the measured peptides with the exception of the two tyrosines still being included (discussed in more detail in section 5.1.1). However, due to the big error of the measurements, no definitive conclusion can be made. The substitution of both tyrosine residues does not completely shut down the ability of the system to perform fusion. One explanation for the significant difference between the measurements are errors linked to the experiment itself. Bulk fusion assays are very susceptible to having a wide distribution of results. Furthermore, other phenomena e.g. aggregation of the particles or interaction with the quartz glass wall are possible. Immobilized vesicles have a lower chance of performing fusion.

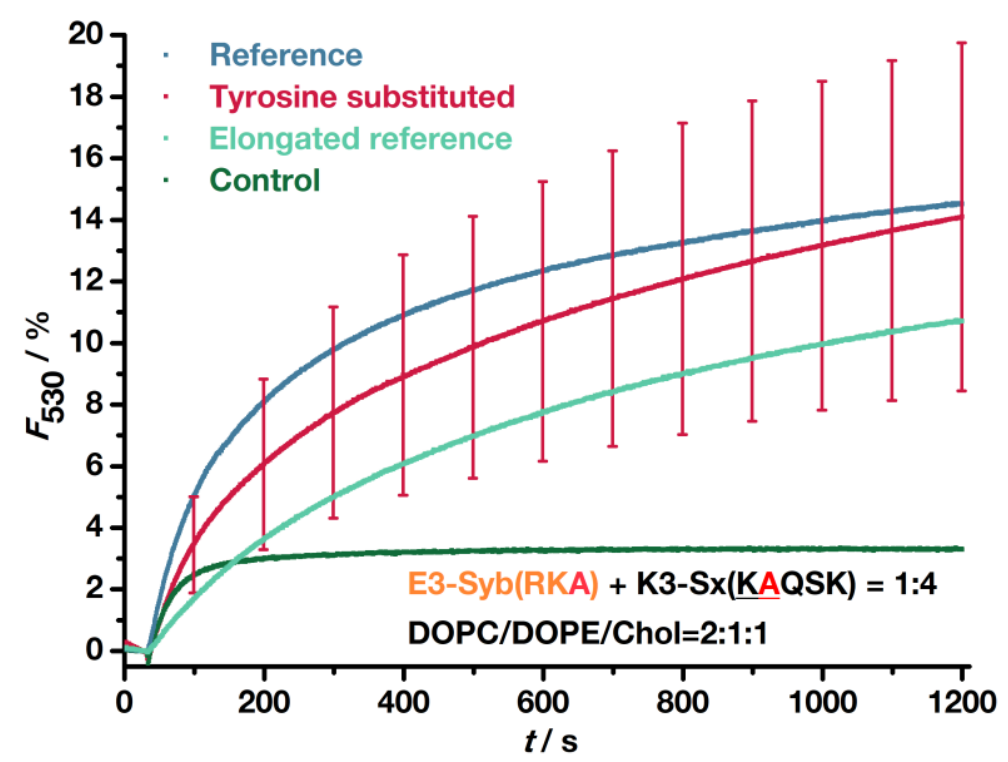

Figure 5.6: FRET dequenching assay of tyrosine substituted peptides. (blue) Standard reference with E3-Syb(RKY) + K3-Sx(QSK). (red) Tyrosine substituted peptides. (teal) Reference of E3-Syb(RKY) + K3-Sx(KYQSK). (green) Control with tyrosine substituted K3-Sx(KAQSK). The average of five measurements with the standard deviation as error bars is illustrated. $\mathrm{P} / \mathrm{L}=1: 500$. 
All vesicles used in the experiments were also analyzed via DLS (Table 5.6). K3-Sx vesicles are bigger than the E3-Syb ones for every measurement. This behavior was observed for every species tested in this work. The mixed population, which was measured 20 min after mixing, showed a higher average size value indicating fusion of at least two vesicles. Indeed, the size increase is expected to be higher. For example, for the first measurement, fusion of a $135 \mathrm{~nm}$ and a $123 \mathrm{~nm}$ vesicle should yield ideally a $162 \mathrm{~nm}$ vesicle. As the average size value is a cumulants mean including all fused and unfused vesicles, this discrepancy is to be expected.

Table 5.6: DLS data of measurements performed for the investigation of the role of tyrosine in the linker.

\begin{tabular}{ccccccc} 
& \multicolumn{2}{c}{ K3-Sx(KAQSK) } & \multicolumn{2}{c}{ E3-Syb(RKA) } & \multicolumn{2}{c}{ Mix } \\
No. & $\begin{array}{c}\text { Z-average } \\
\boldsymbol{d}_{\mathbf{h}} / \mathbf{n m}\end{array}$ & PdI & $\begin{array}{c}\text { Z-average } \\
\boldsymbol{d} / \mathbf{h m}\end{array}$ & PdI & $\begin{array}{c}\text { Z-average } \\
\boldsymbol{d}_{\mathbf{h}} / \mathbf{n m}\end{array}$ & PdI \\
\hline 1 & 135.40 & 0.107 & 123.77 & 0.148 & 150.03 & 0.130 \\
\hline 2 & 133.17 & 0.083 & 130.77 & 0.148 & 155.10 & 0.142 \\
3 & 141.87 & 0.123 & 123.03 & 0.075 & 161.53 & 0.193 \\
4 & 143.47 & 0.177 & 127.77 & 0.063 & 153.00 & 0.129 \\
5 & 136.13 & 0.097 & 124.43 & 0.063 & 145.20 & 0.118 \\
\hline
\end{tabular}

The polydispersity indices behave differently for every measurement, but generally no major changes compared to the two parent vesicles were recorded. Interestingly, between vesicle species of the same kind, mentionable differences were observed. Especially $\mathrm{K} 3-\mathrm{Sx}(\mathrm{KAQSK})$ vesicles show a high variability in the vesicle quality although the peptide was used from the same stock solution. This is also true for the same vesicle population and can be seen in Figure 5.7. Every population is measured three times to form the average value (see section 9.4.5). Normally the standard deviation between the measurements is quite small. However, in these experiments the error was considerably higher, as depicted by the error bars. This behavior was not observed in this magnitude for other experiments, suggesting that the inconsistencies are due to the used peptides. The size distribution clearly shifts to bigger sizes in the mixed sample, signaling fusion or aggregation of the vesicles.

Combining the results of the FRET dequenching assay with the DLS data, interpretation is rather difficult. The high inconsistencies between measurements show a different behavior of the modified peptides compared to other modifications. A comparison between the 
measurement, standard reference and the elongated reference hints, that the high error margin might be due to the tyrosine substitution. Surprisingly, the elongated reference shows a significantly lower intensity increase as well as a different curve form than the tyrosine substituted species. The tyrosines appear to be inhibiting the fusion mechanism of the model system. Though, this observation might also be due to increased destructive aggregation or other phenomena which could also lead to these results. Ultimately, the employed techniques are not sufficient to exclusively explain the obtained results. Nevertheless, they show that the lack of the tyrosines is sufficient to interfere with the fusion mechanism of the model system.

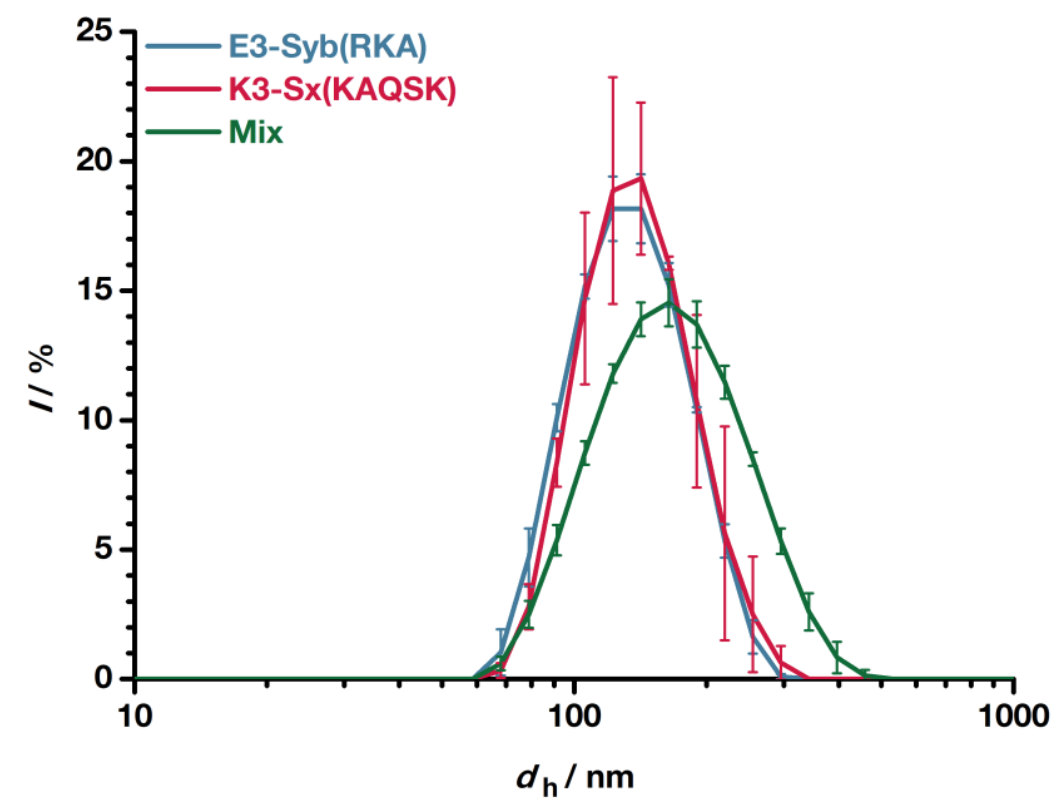

Figure 5.7: Exemplary DLS data of one measurement with E3-Syb(RKA) (blue) and $\mathrm{K} 3-\mathrm{Sx}(\mathrm{KAQSK})$ (red) containing vesicles. The mix curve (green) shows data gathered directly after the FRET dequenching assay.

\subsection{Polybasic Region in Syntaxin 1A}

The polybasic region KARRKK of the syntaxin domain is located in the linker connecting motif and TMD. It is positioned at the junction between polar head groups of the lipid bilayer and the aqueous phase. Due to this location there are multiple interaction partners possible. There are several reports about interaction with specific lipids e.g. $\mathrm{PIP}_{2 .}{ }^{[28,172,240,241]}$ These interactions are presumed to be responsible for the energetics and stability of the membranes. Furthermore, calcium dependent release was impaired in PC12 cells after substitution of this region with alanine. ${ }^{[242]}$ To investigate the role of the lysine 
and arginine residues in the E3/K3-TMD model system, peptides with modifications in the sequence were synthesized and their fusion behavior monitored. The specific substitution pattern was derived from the cis-complex of the native system. As illustrated in Figure 5.8, the lysine residues 260/264 are located near the interface of the two proteins. Interactions with residues of the opposing protein are likely, especially Lys 264 which is in close proximity to Asn 92 and Met 95 of synaptobrevin 2.

(a)

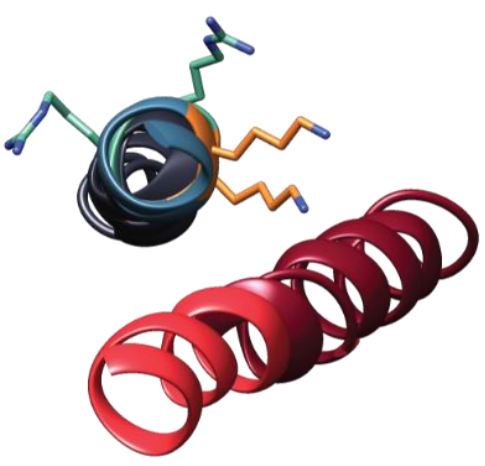

(b)

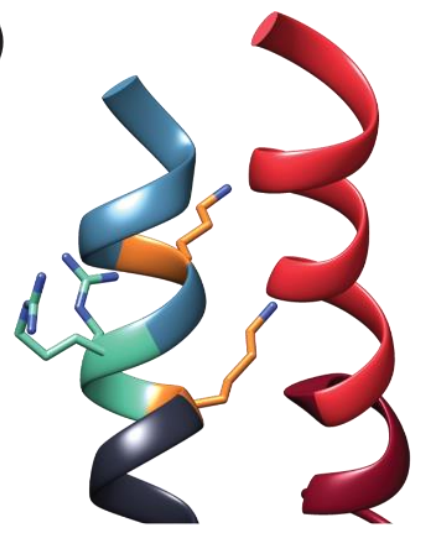

(c)

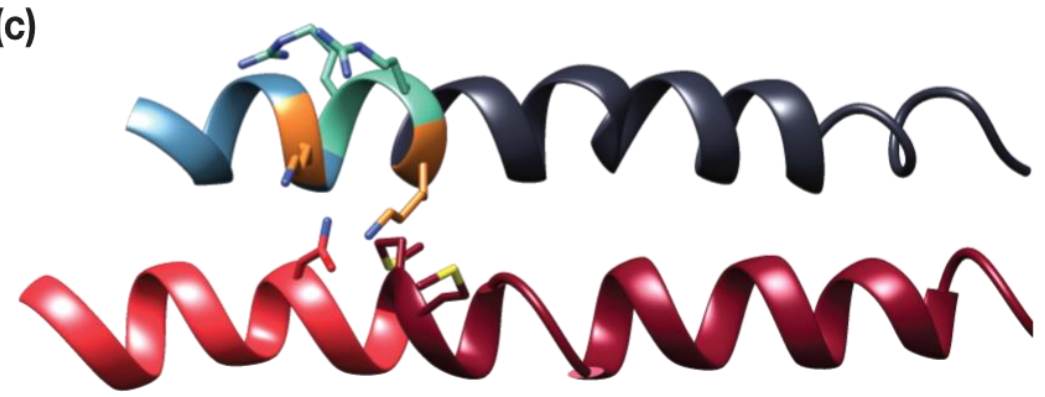

Figure 5.8: Linkers and TMDs of synaptobrevin 2 and syntaxin $1 \mathrm{~A}$ in the post fusion cis configuration. Lysine residues which were modified are marked in orange, synaptobrevin is red and syntaxin is blue. Arginine residues which were substituted for alanine are marked in teal. (a) View from the N-terminal end, (b) view from the side, (c) residues in close proximity to the polybasic area are shown. X-ray data from STEIN et al. ${ }^{[23]}$ Molecular graphics and analyses were performed with the UCSF Chimera package. ${ }^{[121]}$

In contrast to the lysine residues, the arginine moieties are facing the opposite direction in the cis-complex. Embedded into a membrane, these arginines can possibly interact with other proteins or the lipid headgroups surrounding the protein. To investigate if these residues affect the fusion behavior of the E3/K3-TMD model system, two new peptides were synthesized (see Table 5.7). 
Table 5.7: Peptide sequences of reference and modified K3-Sx peptides used for the investigation of the polybasic area in syntaxin. The marked arginines (teal) and lysines (orange) are exchanged with alanine residues.

\begin{tabular}{llc} 
Peptide & Sequence & No. \\
\hline K3-Sx(QSK) & WWG-(KIAALKE) $)_{3}-Q S K A$ KRRK-TMD & (2) \\
K3-Sx(QSKAAAK) & WWG-(KIAALKE) $)_{3}-Q S K A A A K-T M D$ & $\mathbf{( 1 0 )}$ \\
K3-Sx(QSAARRA) & WWG-(KIAALKE) $)_{3}-Q S A A R R A-T M D$ & $\mathbf{( 1 1 )}$ \\
\hline
\end{tabular}

\subsubsection{Role of the Arginine Domain in the Linker of Syntaxin 1A}

To investigate the role of the arginine residues in the linker, both amino acids have been substituted with alanine residues. With this substitution the charge and polarity has been reduced in this area due to the lack of functional groups in the alanine residue. In the used model system, the substitution has a dramatic effect on the fusion efficiency as illustrated in Figure 5.9. The ability of the system to perform fusion is abolished completely. The control experiment consisting of the $\mathrm{K} 3-\mathrm{Sx}(\mathrm{QSKAAAK})$ vesicle and a labeled vesicle is similar in intensity from the start. In contrast, the measurements intensity increases over the course of the experiment more compared to the control. A small amount of interaction between the peptides is therefore possible.

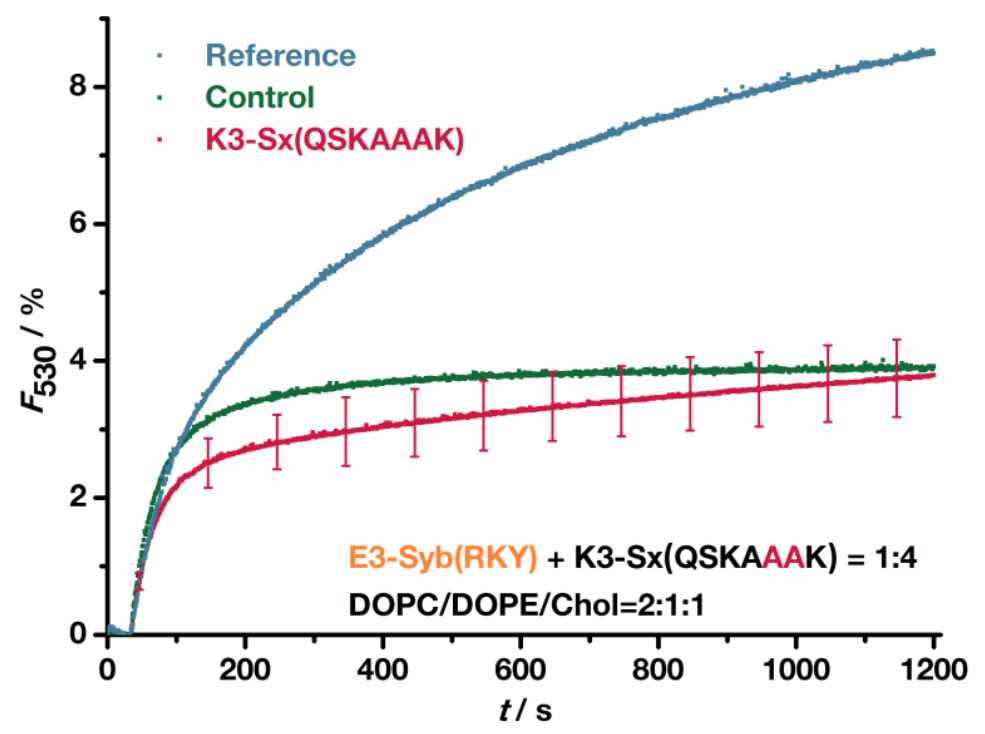

Figure 5.9: FRET dequenching assay of the modified K3-Sx(QSKAAAK) peptide. (blue) reference system. (green) Control measurement. (red) Average of five measurements containing the modified peptide. All measurements were performed with $\mathrm{P} / \mathrm{L}=1: 500$. 
The error of the measurement is noticeably small in spite of being calculated out of five measurements. The used peptide is of an excellent quality as verified via ESI-MS and UPLC, eliminating effects from impurities during the fusion assay. Consequently, the results emphasize the importance of the arginine residues in the syntaxin 1A linker of the model system. To see whether the modified K3-Sx peptide has an effect on the vesicles, DLS data was collected for every measurement and vesicle species (Table 5.8).

Table 5.8: DLS data of vesicle species used in the experiments regarding the arginine domain of syntaxin $1 \mathrm{~A}$.

\begin{tabular}{ccccccc} 
& \multicolumn{2}{c}{ K3-Sx(QSKA_AAK) } & \multicolumn{2}{c}{ E3-Syb(RKY) } & \multicolumn{2}{c}{ Mix } \\
No. & $\begin{array}{c}\text { Z-average } \\
\boldsymbol{d}_{\mathbf{h} / \mathbf{n m}}\end{array}$ & PdI & $\begin{array}{c}\text { Z-average } \\
\boldsymbol{d}_{\mathbf{h}} / \mathbf{n m}\end{array}$ & PdI & $\begin{array}{c}\text { Z-average } \\
\boldsymbol{d}_{\mathbf{h}} / \mathbf{n m}\end{array}$ & PdI \\
\hline 1 & 149.0 & 0.129 & 138.6 & 0.105 & 146.5 & 0.111 \\
2 & 161.1 & 0.099 & 150.0 & 0.127 & 160.5 & 0.081 \\
3 & 157.3 & 0.081 & 148.4 & 0.106 & 160.7 & 0.122 \\
4 & 158.2 & 0.083 & 147.5 & 0.091 & 160.7 & 0.104 \\
5 & 158.9 & 0.088 & 148.6 & 0.108 & 160.6 & 0.102 \\
\hline
\end{tabular}

The initial vesicles are relatively large with $\sim 157 \mathrm{~nm}$ for the K3-Sx vesicles and $\sim 147 \mathrm{~nm}$ for the E3-Syb vesicles. Also, a significant difference between the first and the rest of the measurements is apparent. This inconsistency was caused due to the use of a new batch of polycarbonate membranes which are used during the extrusion process. Unfortunately, there are considerable discrepancies in the quality of these membranes. Due to this, the manufacturer had to be changed, to maintain comparable sizes to previous measurements. Despite the relatively big size difference, the overall trend and observation is the same for all five measurements. The mixed population (post fusion) shows no significant increase in size after being mixed and incubated for $20 \mathrm{~min}$. Usually an increase of $\sim 10 \mathrm{~nm}$ in comparison to the K3-Sx vesicle is detected for fusion. Here, the increase is minimal with $\sim 2 \mathrm{~nm}$.

For the interpretation of these results, many factors have to be considered. The positioning of the arginine residues in the cis-complex facing the lipid environment is ideal for interaction with lipid head groups. As this is the final conformation, it should be low in energy and therefore the desired end state. Nevertheless, the complex has to be formed first to get to the low energy state. The obtained results suggest that the formation did not occur in the first place, otherwise the FRET signal and vesicle sizes would be different. The 
arginines most likely have an effect on the fusion process of the model system prior to the assembly of the complex. The possible effects which explain the observation are manifold. First, the aggregation of the peptide inside the membrane might be altered. It is well known that syntaxin 1A forms clusters in the natural environment. ${ }^{[27,172,243,244]}$ Exchanging the arginines might lead to a different clustering behavior and consequently change crucial properties which are needed for the mechanism. However, in the natural system the substitution of the arginines showed low effect on the binding behavior with PIP $_{2}{ }^{[26,245,246]}$ This trait correlates with the formation of clusters, hence attesting the contrary of this explanation. Yet, the model system has already shown that it behaves quite differently in many other regards. For example, the formation of K3 homodimers has to be considered, which could potentially increase clustering and therefore challenge the results obtained with the natural system. Second, the flexibility of the linker region itself could be improved, as alanine is slightly more flexible than arginine. ${ }^{[45,247]}$ An improved flexibility could mean, that the K3-motif can interact more with the lipid membrane. As it was previously shown, $\mathrm{K} 3$ can attach to the membrane due to the lysines being able to "snorkel" into the membrane. ${ }^{[197,199,202,248]}$ A stronger attachment to the membrane is harder to overcome, thus might lead to less fusion activity of the peptide. Third, due to the substitution the total charge in the linker has changed significantly. The two arginines contribute a net plus two charge toward the peptide. This might interfere with the incorporation of the peptide into the membrane during the extrusion process. The lower the amount of peptide inside the vesicle, the lower the fusion efficiency. Unfortunately, the peptide concentration in the membrane cannot be determined for these types of experiments. The peptides would have to be labeled with a different fluorophore or another group, which would most likely interfere with the ability of the peptide to perform fusion. Furthermore, the substitution of adjacent lysines did not stop fusion completely (see section 5.3.2). Consequently, the loss of the charge is not the conclusive reason for the obtained results.

In summary, substitution of the two arginines drastically changed the peptides ability to perform fusion. This in turn means, that these residues are crucial parts of the model system. 


\subsubsection{Lysines in the Linker of Syntaxin 1A}

Lys 260/264 are part of the polybasic area in syntaxin's linker and are positioned near the interface of the SNARE cis-complex (Figure 5.8). To see whether the lysines are important for the model systems performance regarding fusion, a peptide species with substituted alanines was synthesized (see Table 5.7).

The results from the FRET dequenching assay show a reduction in fusion efficiency due to the substitution of the lysines 260/264 (see Figure 5.10). In comparison to the reference the measurements show a decrease in total intensity of $30 \%$ after $20 \mathrm{~min}$. The standard deviation, displayed by the error bars, is reasonably low. Toward the 10 min mark the error is smaller compared to the beginning and end of the experiment. This can be explained due to the various single measurements showing a different slope of the fusion curves. Coincidentally, some of the measurements intersect at the 10 min mark. A peptide specific reason for this observation is highly unlikely as this was not noticed for any other species before. Furthermore, the curve shapes of all measurements do not show a kink or other anomalies. The control measurement consisting of a labeled vesicle with an unlabeled K3-Sx(QSAARRA) vesicle shows a typical curve shape and intensity.

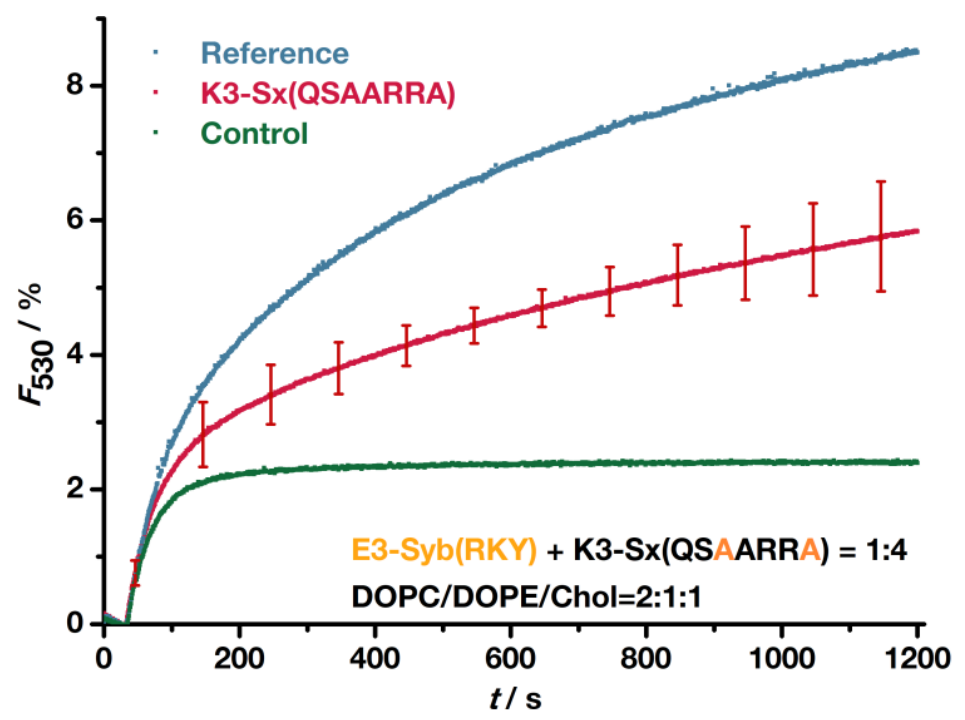

Figure 5.10: FRET dequenching assay of the modified K3-Sx(QSAARRA) peptide. (blue) Reference. (red) Average of five measurements containing the modified peptide. (green) Control. All measurements were conducted with $\mathrm{P} / \mathrm{L}=1: 500$. 
Comparing all three curves of Figure 5.10, several conclusions regarding the lysines 260/264 can be drawn. First, substitution of both lysines with alanine did not abolish the peptides ability to perform fusion. This can be clearly seen by comparing the measurements with the control. The lysines do not appear to be essential for the model system but do increase the efficiency of this process. Neither the loss of the two positive charges in the membrane headgroup area nor the possible missing interactions of the two residues with synaptobrevin or lipids is sufficient to render K3-Sx(QSAARRA) incapable of fusion. In regard to the initial assumption that the lysines might interact with synaptobrevin, hence stabilizing the complex, it is likely that these interactions are not the driving force of this process. Indeed, it is assumed that the main function of the polybasic area is the interaction with lipids, especially $\mathrm{PIP}_{2}$ and the resulting formation of syntaxin clusters. ${ }^{[24,27,28,249]}$ Particularly lysines 264/265 have been found to be effective at binding PIP $2 .{ }^{[26]}$ However, as no charged lipids (except the labeled lipids) were used during the experiment, this specific function of the lysines was of no need. Therefore, the reduced fusion efficiency might still be due to minimal interaction between the two peptides. Results of the accompanying DLS measurements are shown in Table 5.9.

Table 5.9: DLS data of vesicle species used in the experiments regarding the lysines of the linker of syntaxin.

K3-Sx(QS吕ARA) $\quad$ E3-Syb(RKY) $\quad$ Mix

\begin{tabular}{ccccccc} 
No. & $\begin{array}{c}\text { Z-average } \\
\boldsymbol{d} \mathbf{h} / \mathbf{n m}\end{array}$ & PdI & $\begin{array}{c}\text { Z-average } \\
\boldsymbol{d} \mathbf{h} / \mathbf{n m}\end{array}$ & PdI & $\begin{array}{c}\text { Z-average } \\
\boldsymbol{d} \mathbf{h} / \mathbf{n m}\end{array}$ & PdI \\
\hline 1 & 152.4 & 0.132 & 128.3 & 0.115 & 152.9 & 0.127 \\
2 & 162.7 & 0.114 & 148.7 & 0.111 & 185.4 & 0.138 \\
3 & 159.4 & 0.107 & 134.6 & 0.105 & 170.3 & 0.124 \\
4 & 163.3 & 0.093 & 134.6 & 0.105 & 173.3 & 0.103 \\
5 & 157.4 & 0.083 & 141.2 & 0.116 & 169.3 & 0.114 \\
\hline
\end{tabular}

Measurements 3-5 show normal vesicle sizes and size development after fusion. The first measurement does not show a size increase and the second presents an unusually high increase in the mixed population. In combination with the individual FRET dequenching curves (not shown) there is no obvious correlation. All measurements except measurement 5 show a fairly identical curve shape. All in all, the modified K3-Sx(QSAARRA) is handicapped in its ability to perform fusion compared to the reference. If the initial assumption of these lysines being involved in intermolecular peptide to peptide interactions 
is true, the lysines contributions are important, but not as essential as the arginines (see section 5.3.1). Indeed, the reason for this behavior can not be determined with just the employed techniques. However, one of the main differences between the two lysines and the arginines is their positions in the peptide. The charge should be the same at $\mathrm{pH}=7.40$ as both amino acids have $\mathrm{pK}$ values for the ionizable groups well over 9. ${ }^{[250]}$ Consequently, if the ionic interactions are the reason for the change in fusion behavior, it is most likely because of the charges position in the peptide.

In the end, the lysines seem to be suitable points of modification, as the peptides functional ability is not completely inhibited. These results are the basis for the mechanistic investigations as described in chapter 6 . 


\section{Photocaging of K3-Sx}

The zippering hypothesis is the most accepted theory regarding the membrane fusion mechanism of SNARE complexes. ${ }^{[168]}$ One of the initial reasons for the design of the E3/K3-TMD model system was to maintain the peptidic backbone to study the zippering mechanism. ${ }^{[22]}$ To investigate this process, numerous experiments can be performed. One approach is to stop the process at a specific point and starting it again upon a specific trigger. This method was chosen for investigating the mechanism in the E3/K3-TMD model system.

Briefly, the interactions between the peptides should start at the N-termini with the formation of the E3/K3 coiled coil, similar to the natural system. At some point the interaction either of the motif, linker or TMD is encumbered by means of steric hindrance, arresting the peptides in a state where the C-terminal parts are not engaging in contact with each other. A trigger, e.g. $\mathrm{pH}$ - or temperature change, light or specific chemicals releases the obstruction, continuing the formation of the complex and ideally leading to membrane fusion. In this chapter the specific experiment and the peptide requirements are elucidated (section 6.1). Further, the design specifications of the caging group and the synthesis are discussed in section 6.2. Ultimately, the results of the experiments are presented (sections 6.3 and 6.4)

\subsection{Design of the Photocaged Peptide}

The aim of the introduction of the photo caging group is to get more insight into the mechanism of the fusion process of the E3/K3-TMD system. This can be achieved by the introduction of these groups at specific positions in the peptide sequence (see Figure 6.1). The cage is inserted in the linker region of one of the peptides, more specifically in K3-Sx. The initial interactions of the two motifs are not encumbered by this. The coiled coil at the $\mathrm{N}$-terminal end can form, but stops in the middle of the peptide at the linker region. The cage sits in between both peptides, making it sterically impossible for more interactions. At this point, both vesicles are in close proximity but arrested in a primed like state. Similar 
to the natural system, where the influx of $\mathrm{Ca}^{2+}$ starts the fusion step, a trigger is applied. In this case, light of $\sim 400 \mathrm{~nm}$ starts the rearrangement of the photo active group, leading to the elimination of the steric group. The interaction of the peptides continues, and membrane fusion is facilitated. This process can be observed via FRET dequenching assays, as one of the vesicles is equipped with a FRET pair.

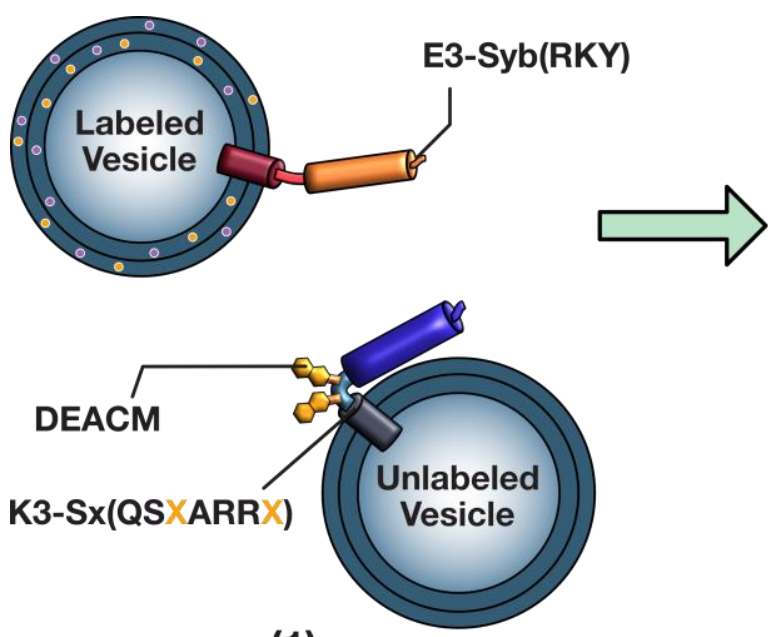

(1)

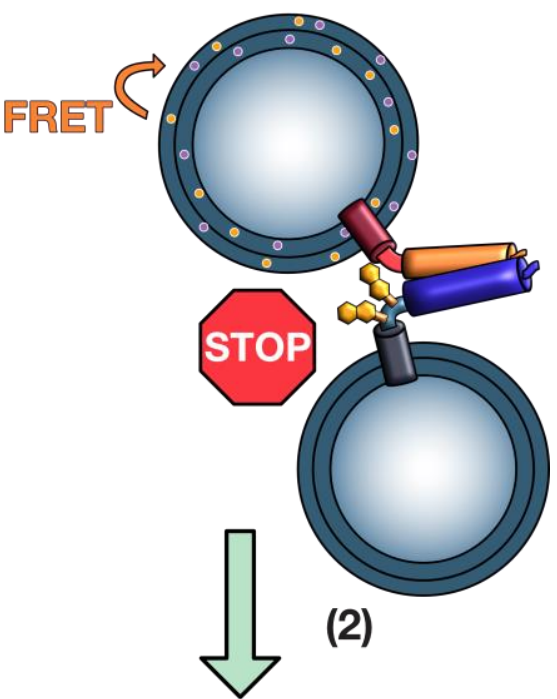

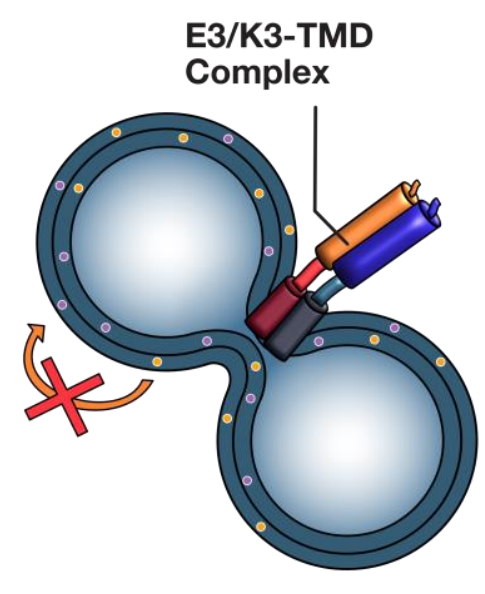

(4)
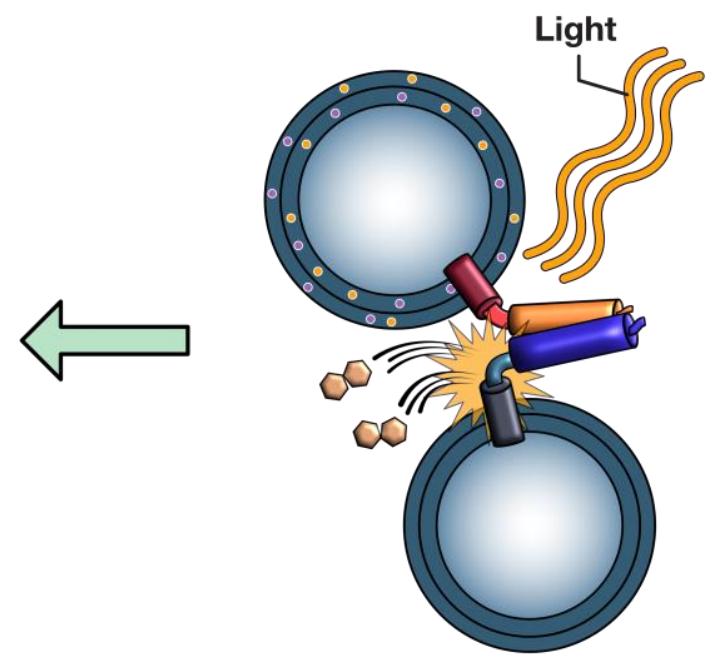

(3)

Figure 6.1: Schematic depiction of the experiment employed for the investigation of the fusion process of the E3/K3-TMD model system. DEACM is the used photocage. (1) The two peptides E3-Syb(RKY) and K3-Sx(QSXARRX) are incorporated in opposing vesicles. (2) Interaction of the motifs starts at the $\mathrm{N}$-terminal end, pulling the vesicles closer to each other. The zippering stops after the caging groups are reached. (3) The peptides stay in the ready state until light of suitable wavelength cleaves the photocages off. (4) The peptides proceed with the pore opening, leading to the fusion of both vesicles. A positive signal in the FRET dequenching assay can be observed as a result of the successful fusion. 
To perform an experiment where the fusion process is stopped in the middle, special peptides had to be synthesized. Here, the obstruction was introduced into the linker region of K3-Sx (Table 6.1). Two lysines in the polybasic area, namely Lys 260 and Lys 264 of the natural syntaxin $1 \mathrm{~A}$ were chosen, as they showed to be important for the fusion of this system (see section 5.3.2).

Table 6.1: Comparison between the reference K3-Sx peptide and the caged species sequence. The syntaxin 1A TMD sequence is omitted.

\begin{tabular}{llc} 
Peptide & Sequence & No. \\
\hline K3-Sx(QSK) & WWG-(KIAALKE) ${ }_{3}-Q S K A R R K K-T M D$ & (2) \\
K3-Sx(QSXARRX) & WWG-(KIAALKE) ${ }_{3}-Q S X A R R X K-T M D$ & (5) \\
\hline
\end{tabular}

Arguably, the adjacent arginine domain seems to have an even bigger impact on the fusion behavior of the model system. However, there are two reasons why the substitution of the two lysines was preferred for the mechanistic study. First, in the natural SNARE cis-complex the arginines point away from the interface of the two proteins, whereas the lysins are near it (see Figure 6.2). Assuming that the configuration in the model system is similar to nature during the fusion process, the lysins are the preferred point of modification. Second, the target component which is used for steric hindrance was already synthesized and successfully incorporated into the model system. ${ }^{[219]}$ The building block, which consists of a lysine with a coumarin based photocleavable group in the sidechain, was already established in several other experiments and proved to be reliable. ${ }^{[219]}$

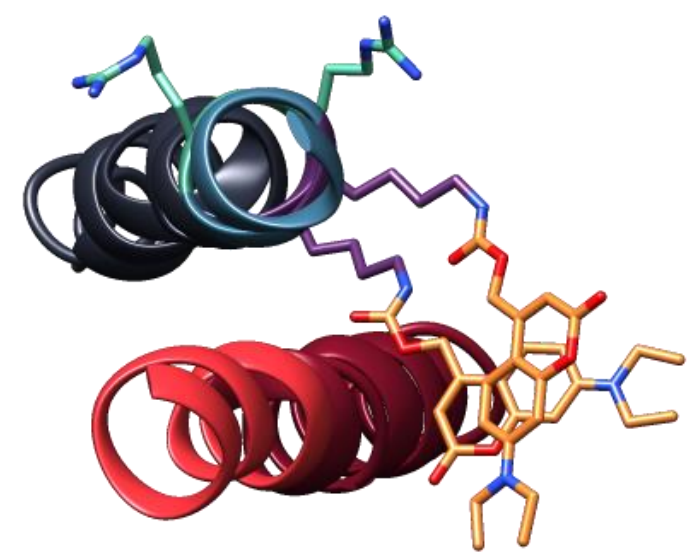

Figure 6.2: Illustration of a composition of the caged linker region of syntaxin 1A with DEACM. The DEACM cage is marked in orange. Lys 260/264 are marked in purple and Arg 262/263 are marked in teal. X-ray data from STEIN et al. ${ }^{[23]}$ Molecular graphics and analyses were performed with the UCSF Chimera package. ${ }^{[121]}$ 
To increase the steric hindrance and therefore ensure the stop of the fusion process, both lysines were modified simultaneously. With two bulky groups in slightly different positions, the effectiveness is increased due to more spatial coverage. Also, the X-ray data of the cis-complex does not necessarily reflect the situation in the model system. As described in chapter 5.1, the model system might be in a slightly other configuration compared to the natural system. Therefore, the positions of the two lysine residues might be aligned in another way.

In case of synaptobrevin 2, there are also two lysine residues namely Lys 85/91 in the linker which might be suitable candidates for modification (see Figure 6.3). However, Lys 85 is not included in the model system as the artificial motif is attached to Arg 86 of the synaptobrevin linker. Addition of the extra amino acid might interfere with the rest of the system as a sequence shift would be implied (see also section 5.1).

(a)

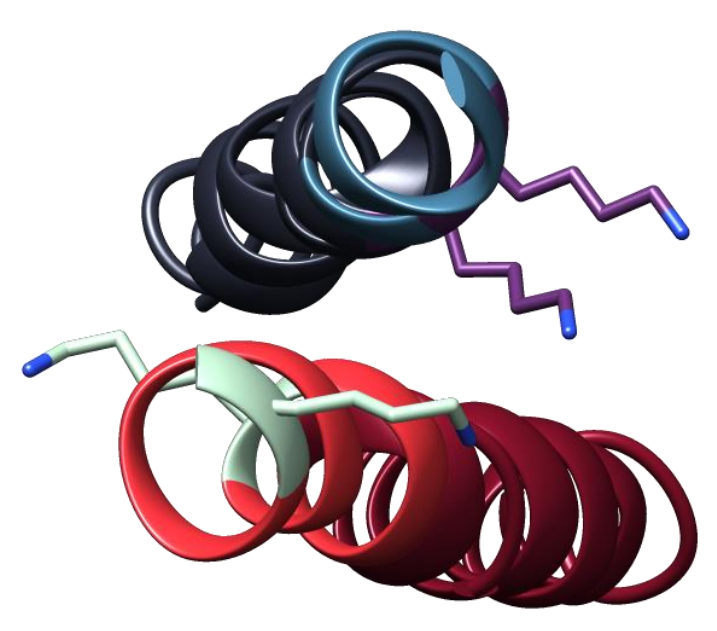

(b)

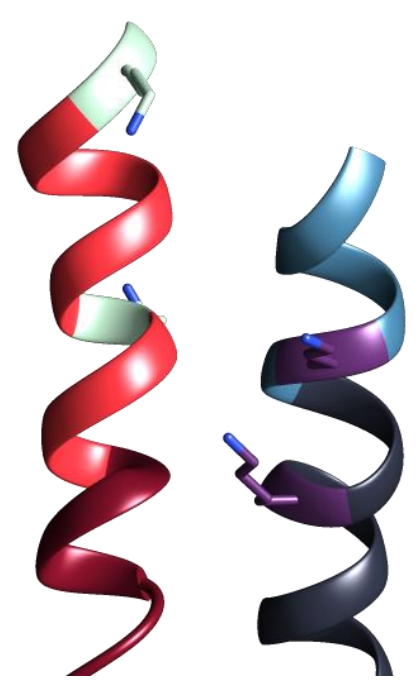

Figure 6.3: Marked lysines in the linkers and TMDs of synaptobrevin 2 and syntaxin 1A in the post fusion cis-configuration. Lysine residues in syntaxin (blue) which were modified with the photocage are marked in purple. Mint green marked lysines in synaptobrevin 2 (red) are possible candidates for caging groups. (a) View from the top. (b) View from the side. X-ray data from STEIN et al. ${ }^{[23]}$ Molecular graphics and analyses were performed with the UCSF Chimera package. ${ }^{[121]}$ 


\subsection{Synthesis of the Photocaged Peptide}

The group which is used to stop the annealing has to have specific properties. First, it has to be big enough to prevent the interaction of the peptides. A small group might interfere, but due to the length of the peptides would not be able to stop the process. Second, it should not interact with other amino acid residues, lipids or other parts of the system. A charged group, for example, could interact with the other peptide strongly, making it difficult to leave and therefore keep interfering with the process. Additionally, products formed during cleavage should also follow these criteria. These molecules have a high movability as they are not bound covalently to anything, making them prime candidates for unwanted interactions. Third, cleaving the group should be time efficient. The fusion process itself is fast and thus observation of this event would not be possible using a group which needs too much time during cleavage. Also, the more time is used, the less information can be gathered regarding fusion kinetics. Fourth, the outgoing group must be stable for use in SPPS. The incorporation of the group has to be done on the amino acid building block prior to peptide synthesis. The peptides of the model system are about 50 amino acids long. Introducing the group after peptide synthesis is far more challenging due to the many different amino acid residues present. Also, steric hindrance because of the peptide length could interfere with the yield of the reaction.

For the caging group 7-Diethylamino-4-methylcoumarin (DEACM) has been selected. DEACM is one of the fastest cages currently known. ${ }^{[251]}$ The quantum yields are high and the excitation wavelength is around $400 \mathrm{~nm}$, making it a prime candidate for incorporation into a peptide. ${ }^{[252]}$ Additionally, the group was already modified for the use on amines and successfully tested in bacterial and mammalian cells. ${ }^{[253]}$ Furthermore, the photo group was already tested in the MEYENBERG model system. ${ }^{[219]}$ A modified Lys-(DEACM) amino acid was used in the artificial motif to stop the zippering process. Due to their positioning in the $g$ and $e$ position of the coiled coil, the steric hindrance was too low resulting in no inhibition of the process. ${ }^{[219]}$

Synthesis of the building block was successful following the procedure of ZHANG and SCHIRMACHER (Figure 6.4). ${ }^{[219,254]}$ Briefly, 7-Diethylamino-4-methylcoumarin (12) was oxidized with $\mathrm{SeO}_{2}$ and subsequently reduced to the alcohol 13. The alcohol was then activated with 4-nitrophenyl chloroformate to anhydride 14. The anhydride was then 
converted by nucleophilic substitution with Fmoc-L-Lys-OH to form the desired Fmoc-LLys(DEACM)-OH building block 15.

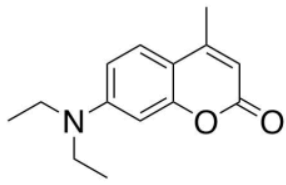

12

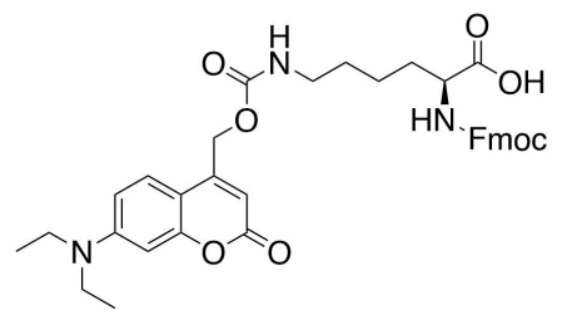

15 a) $\mathrm{SeO}_{2}$, Xylene, $145^{\circ} \mathrm{C}, 48 \mathrm{~h}$

b) $\mathrm{NaBH}_{4}, \mathrm{MeOH}, \mathrm{rt}, 24 \mathrm{~h}$ $42 \%$

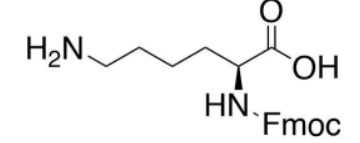
DIPEA

DMF, DCM, Toluol $24 \mathrm{~h}, 0^{\circ} \mathrm{C} \rightarrow \mathrm{rt}$ $77 \%$<smiles>CCN(CC)c1ccc2c(CO)cc(=O)oc2c1</smiles>

13

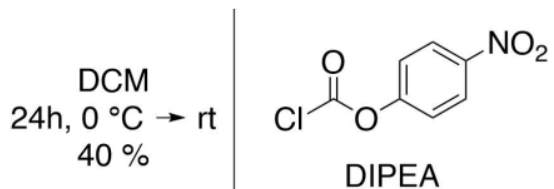<smiles>CCN(CC)c1ccc2c(COC(=O)Oc3ccc([N+](=O)[O-])cc3)cc(=O)oc2c1</smiles>

14

Figure 6.4: Synthesis route of the photo cage Fmoc-L-Lys-(DEACM)-OH. In the first step, 12 is oxidized and subsequently reduced to the alcohol 13 . Next, activation of the alcohol is performed with 4-nitropheyl chloroformate to the anhydride 14. In the last step, a nucleophilic substitution with the amine of FmocL-Lys-OH leads to the target structure 15.

The incorporation of the Fmoc-Lys-(DEACM)-OH building block into the peptide was done during automated peptide synthesis (see chapter 9.3). Owing to the fact that the building block had to be synthesized over several days, the coupling method was modified slightly compared to the other amino acids. The concentration was lowered but the reaction time was increased to ensure a good coupling yield. Because of the lower concentration of the building block, double coupling was performed for both positions, increasing the efficiency even further. Subsequent coupling steps of standard amino acids were performed as usual. After peptide synthesis was finished, every following step was done under the exclusion of light to minimize unwanted uncaging. Cleavage from resin and the workup procedure were not altered compared to the other peptides. The quality was checked using UPLC and mass analysis. 


\subsection{FRET Dequenching Assays of DEACM caged Peptide}

To test if the two cages are capable of stopping the fusion process, different FRET dequenching assays have been performed. One experiment consisted of the standard E3-Syb and K3-Sx(QSXARRXK) vesicles being mixed in the fluorescence cuvette, following the standard procedure (Figure 6.5). Due to the bulky cage, fusion is inhibited, as can be seen in the first $300 \mathrm{~s}$. A small increase in signal intensity was observed, but this might be due to unspecific interaction of the K3-Sx peptide with the other vesicle as already discussed in section 4.3.4. After $300 \mathrm{~s}$ the sample was taken out of the fluorescence spectrometer and irradiated with a laser of $400-415 \mathrm{~nm}(75 \mathrm{~mW})$ for $60 \mathrm{~s}$. During this time, no data points could be obtained. Subsequently, the cuvette was put back into the spectrometer and the measurement was continued. In the first few seconds, a small irregular increase followed by an intensity drop was observed. This is most likely due to the stirring of the sample starting again after inserting the cuvette into the spectrometer. After that, data points with a significant increase in intensity compared to the first $300 \mathrm{~s}$ were gathered. These results indicate the successful uncaging of the K3-Sx peptide and the subsequent fusion process between the two vesicle species. Arguably, the exact mechanism cannot be derived from this experiment as there are multiple feasible explanations for this behavior. First, the mechanism could follow the intended way of the motifs forming a coiled coil but not being able to perform the fusion process. This hypothesis is supported by the fact, that the increase after the first laser session is as pronounced as observed. The system would be in a primed state which formed in the first $300 \mathrm{~s}$ of the experiment, waiting for the trigger to continue the fusion. A second possible explanation is, that there is no interaction of the two peptides up until the uncaging. The fusion mechanism would start from the beginning directly after cleaving of the cage. The steepest intensity increase in dequenching assays can be observed in the first seconds after mixing the two vesicle species. Yet, in comparison to similar experiments the increase jump is much steeper. Most likely a combination of both hypothesis is the cause of these findings. The "primed" system is in equilibrium with the two free peptides. Consequently, a significant number of vesicles is ready to fuse with each other. 


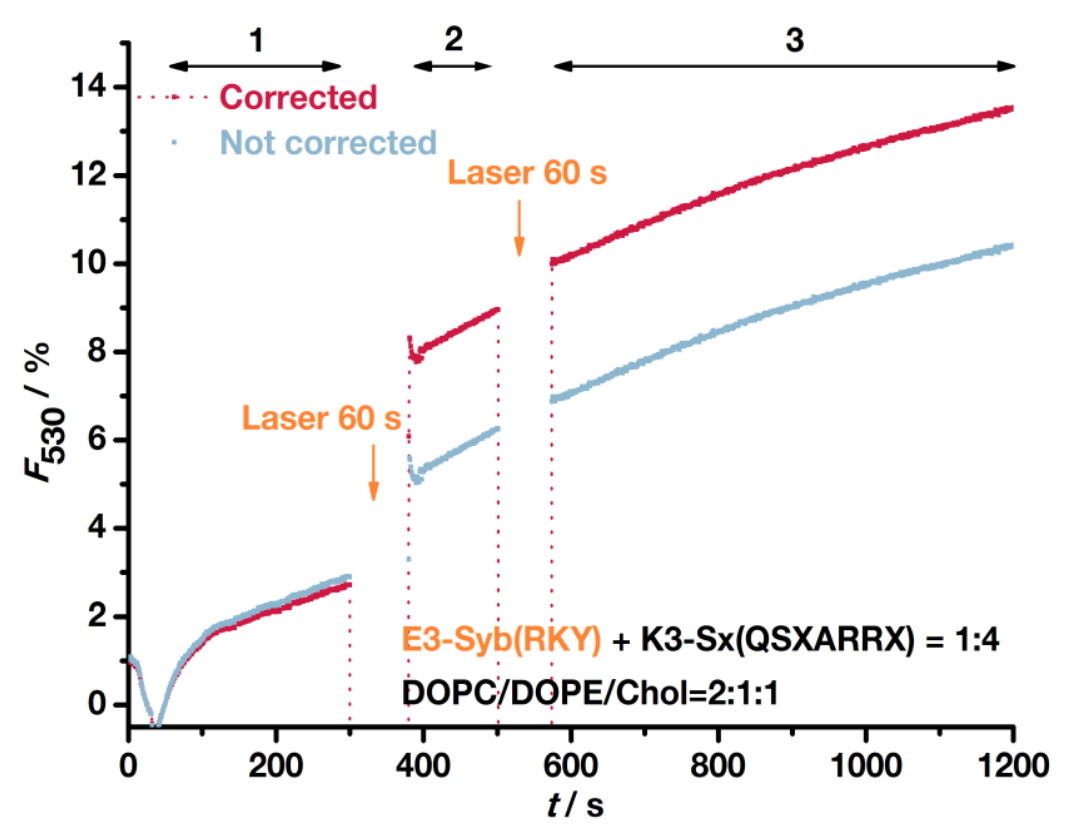

Figure 6.5: FRET dequenching assay with the caged K3-Sx peptide. (red) Curve corrected for photo bleaching and fluorescence increase by uncaging. (blue) Curve without corrections. At $300 \mathrm{~s}$ and $500 \mathrm{~s}$ the sample was taken out of the detector and irradiated with laser light of 400-415 nm for $60 \mathrm{~s}$ each. The numbers on top show the sections, where different amounts of laser irradiation were applied to the vesicle population.

To ensure that most of the caging groups were cleaved, the sample was irradiated a second time for $1 \mathrm{~min}$ at $500 \mathrm{~s}$. The increase in intensity is much lower compared to the first time. This indicates that the majority of the caging groups are cleaved off after the first $60 \mathrm{~s}$. These results are coherent with previous findings using similar peptides. ${ }^{[219]}$ Furthermore, DLS data was acquired for this experiment (Table 6.2). The E3-Syb vesicle shows typical average size and PdI values. The K3-Sx species shows an unusually small PdI but otherwise the size is comparable to other vesicles with different K3-Sx peptides. The mixed species is $8.6 \mathrm{~nm}$ bigger than the $\mathrm{K} 3-\mathrm{Sx}$ vesicle, supporting the observations of the dequenching assay of successful fusion. It was measured at the end of the experiment after both laser irradiation periods. However, determining the sizes between the different stages of irradiation without interfering in the lipid mixing assay is impossible. The DLS measurements take between 5-7 min at which time no fluorescence data can be obtained. On basis of these results, all following experiments with the caged peptides were conducted using a photo cleaving time of $60 \mathrm{~s}$. 
Table 6.2: DLS data of vesicle species used in the uncaging experiment.

\begin{tabular}{lcc} 
Peptide & $\begin{array}{c}\text { Z-Average } \\
\boldsymbol{d}_{\mathbf{h}} / \mathbf{n m}\end{array}$ & PdI \\
\hline E3-Syb(RKY) & 133.3 & 0.072 \\
K3-Sx(QSXARRX) & 148.0 & 0.058 \\
Mix & 156.6 & 0.906 \\
\hline
\end{tabular}

This type of measurement turned out to be quite important for understanding the behavior and handling of the caged peptide. First, in this measurement the whole sample was irradiated with the laser. This included the fluorophores present in the E3-Syb vesicles. For this reason, it had to be tested what effect the laser has on the fluorophores as photobleaching is a common phenomenon among this group of chemicals. To investigate this, measurements have been done using the same amount of E3-Syb vesicles and applying different durations of laser light to it (Figure 6.6).

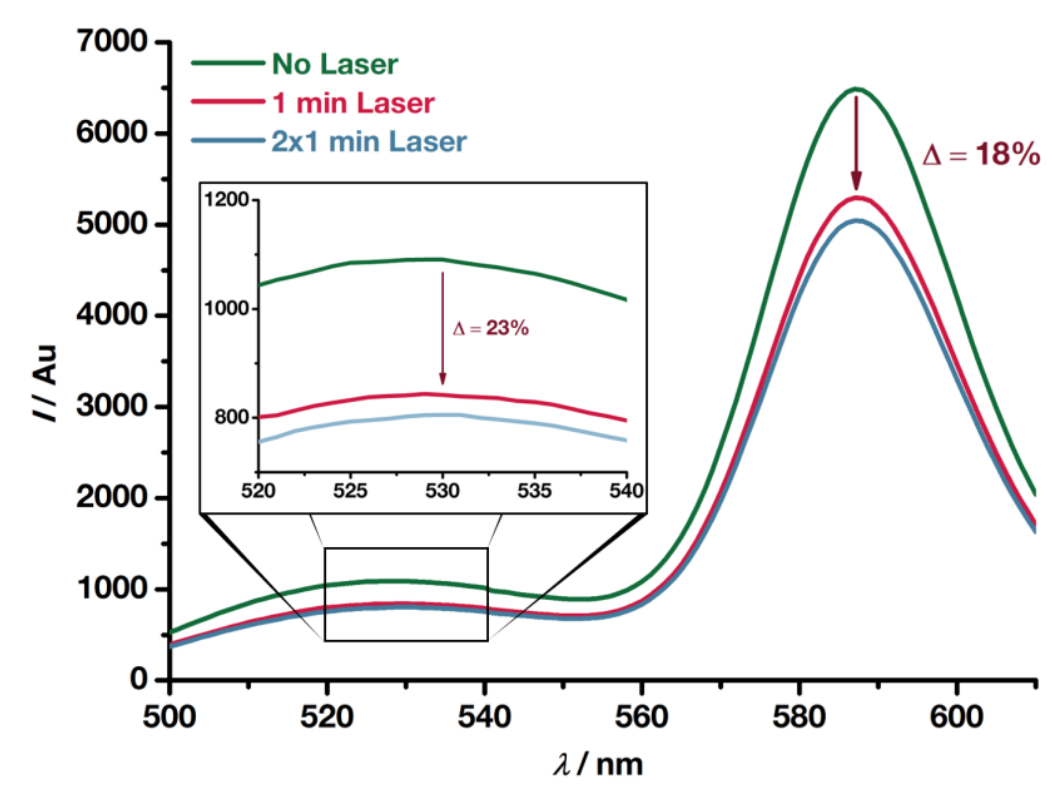

Figure 6.6: Fluorescence emission spectrum with excitation at $460 \mathrm{~nm}$ of E3-Syb vesicles. (green) Emission without laser. (red) Emission after $60 \mathrm{~s}$ laser irradiation. (blue) Emission after $120 \mathrm{~s}$ irradiation. The laser has a range of 400-415 nm with $75 \mathrm{~mW}$.

The emission maximum of Rhodamine at $587 \mathrm{~nm}$ decreases significantly after $60 \mathrm{~s}$ laser irradiation by $18 \%$. After subsequent $60 \mathrm{~s}$ the decrease is much smaller but still noticeable. The laser radiation is, as expected, contributing to photobleaching. As the time course measurements were recorded at $530 \mathrm{~nm}$, the change in intensity at this wavelength was quantified. Here, the decrease was even stronger with $23 \%$ after 60 s. As a result of this, 
for each section a data correction value, which was derived from Figure 4.6 was added to take this effect into account (red curve in Figure 4.5). Furthermore, different maximum values, which are recorded after the addition of TritonX-100, had to be set due to different amounts of photo bleaching in the three sections of the experiment (see chapter 9.4.4 for more detail).

In addition to the photo bleaching, a second phenomenon was observed. After cleavage of the photo group, the fluorescence intensity at $530 \mathrm{~nm}$ increased depending on the amount of cage present (Figure 6.7). This jump in intensity is visible for all dequenching assays where the uncaging occurred. The reason for this anomaly is most likely the fluorescence of the free DEACM-OH which is a result of the cleaving process. Although the excitation maximum for this group is at around $385 \mathrm{~nm}$ depending on the solvent, a small fraction is also absorbed at $460 \mathrm{~nm} \cdot{ }^{[255,256]}$ Emission at $530 \mathrm{~nm}$ is possible and could therefore be detected in this assay. Consequently, all the measurements were corrected to accommodate to this effect. For the ones depicted in Figure 6.5 two separate corrections for section two and three were performed, as the intensity increase is proportional to the amount of the cleaved group. In regard to the impact of this effect on the overall measurement, it is roughly a 3-4 \% increase in fusion efficiency which in the scale of these experiments is a significant amount. In comparison, photo bleaching reduces the intensity by 5-7\%. Combining both effects, the values were effectively increased by $\sim 2.5 \%$ fusion efficiency, making the jump in intensity from section one to two bigger compared to the uncorrected data.

Because of the many different phenomena in this experiment, the results have to be treated with caution. The gathered information is merely showing the proof of concept as it is also noticeable in the uncorrected curve of Figure 4.5. In the end, the adjustment of the data is necessary to emphasize the jump in fusion efficiency because of the removal of the steric obstruction. Furthermore, the adjusted values are more in line with the results from the experiments obtained in section 6.4. 


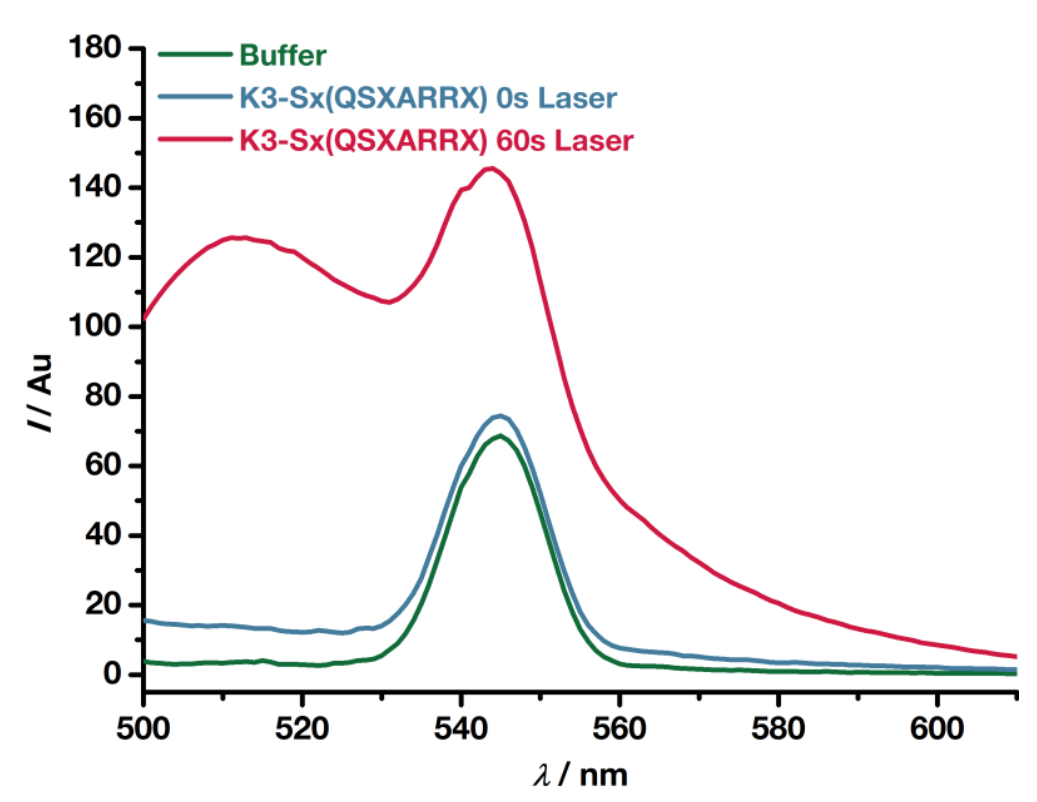

Figure 6.7: Fluorescence emission spectrum with excitation at $460 \mathrm{~nm}$ of K3-Sx(QSXARRX) vesicles with different amounts of laser exposure. (red) Spectrum after $60 \mathrm{~s}$ of laser irradiation. (blue) Spectrum with no laser exposure at the standard concentration in buffer. (green) Spectrum of HEPES buffer.

To verify if the peptide is uncaged completely after $60 \mathrm{~s}$, the efficiency was monitored via UPLC (Figure 6.8). Due to the high UV/Vis absorption of the caging group at $380 \mathrm{~nm}$, the allocation of the peaks is fairly easy. Furthermore, the identities of the peaks were confirmed by LC-MS measurements. The initial chromatogram of the K3-Sx(QSXARRX) peptide without exposure to the laser shows the main peak at $15.1 \mathrm{~min}$ which corresponds to the double caged peptide. Traces of cleaved DEACM-OH at $5.8 \mathrm{~min}$ are visible, indicating that a small percentage of the peptide is not caged fully. This assumption is further supported by the small peak at $14.5 \mathrm{~min}$ which is most likely a single caged peptide. However, the LC-MS data was not conclusive for this peak.

After $30 \mathrm{~s}$ of laser exposure, the main peaks intensity is significantly reduced. Several other peaks appear, which are side products of the photo cleaving step. These were not investigated further due to ambiguous MS data. The intensity ratio between all peaks changes only slightly after $60 \mathrm{~s}$ and $120 \mathrm{~s}$ exposure to the laser. 


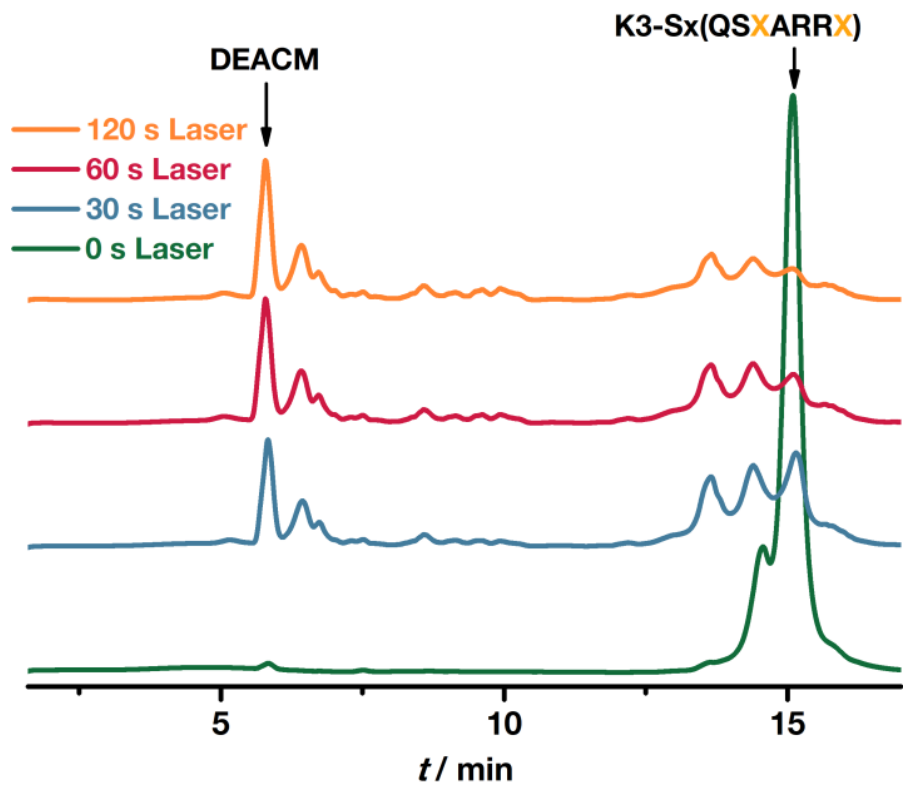

Figure 6.8: UPLC chromatograms of K3-Sx(QSXARRX) after different amounts of laser exposure. (yellow) After $120 \mathrm{~s}$ laser exposure; (red) $60 \mathrm{~s}$ laser exposure. (blue) $30 \mathrm{~s}$ laser exposure. (green) No laser exposure. Data was acquired with ACE Excel 2 C18, 65-95\% B in $18 \mathrm{~min}$ at $380 \mathrm{~nm}$.

All in all, the irradiation of the peptide with the laser leads to successful uncaging of the peptide. The peak at $15.1 \mathrm{~min}$ decreases in intensity whereas the amount of DEACM increases. Really, more than $30 \mathrm{~s}$ of irradiation did not alter the uncaging process. Some unidentified artefacts are formed which may interfere in the fusion assay. Also, it cannot be ruled out, that both cages are cleaved off of every peptide or that the free cage interacts with the peptide. Due to these observations, the chosen $60 \mathrm{~s}$ of irradiation for the fusion experiments is adequate.

\subsection{Effect of the Photocage on Fusion Efficiency}

Parts of the following experiments were done during Fehmke Reinhardt's bachelor thesis under the supervision of Mike C. Groth. ${ }^{[235]}$

The previously discussed experiments were complicated to interpretate, as several factors had a significant impact on the results. To reduce the complexity concerning data adjustments, the following FRET dequenching assays were performed in another way. Instead of the uncaging of the K3-Sx peptide with the labeled E3-Syb vesicles present, the cleavage was carried out before both populations were mixed (for experimental detail see 
section 9.4.4). The freshly extruded K3-Sx vesicle solution was treated with laser light directly before insertion to the cuvette at the $30 \mathrm{~s}$ mark. Ideally, a solution consisting of the standard K3-Sx(QSK) vesicles and the cleaved DEACM-OH group was added to the labeled E3-Syb vesicles. This implies full uncaging of the photo group. Therefore, all data acquired using this method, was corrected by the intensity value produced because of the emission of the free DEACM-OH (see Figure 4.7). For this, the fluorescence of the K3-Sx sample at $530 \mathrm{~nm}$ was recorded prior to laser treatment and compared to the value after the procedure. The difference between the values was subtracted from the measurements. On average, a difference of $\sim 125 \mathrm{Au}$ was measured and consequently subtracted from the data points. The results of these measurements are shown in Figure 6.9.

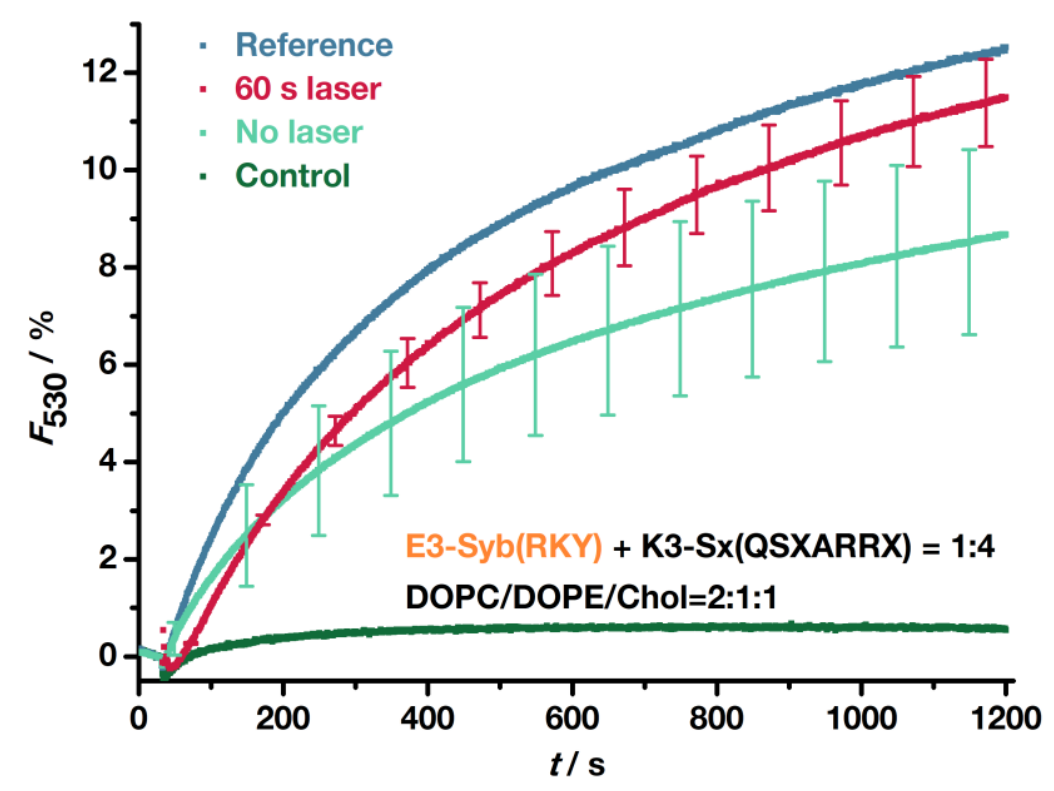

Figure 6.9: Comparison of different K3-Sx vesicle species in FRET dequenching assays. (blue) Reference vesicles containing K3-Sx(QSK) and E3-Syb(RKY). (red) K3-Sx(QSXARRX) vesicles after $60 \mathrm{~s}$ of irradiation with the laser. (teal) K3-Sx(QSXARRX) vesicles without laser. (green) Control measurement containing both K3-Sx(QSXARRX) and labeled vesicles.

Uncaging the K3-Sx species prior to the introduction to the other vesicle population was successful. The fusion efficiency obtained after $20 \mathrm{~min}$ reaches about $11 \%$ and falls short slightly behind the reference. However, these results have to be treated carefully as the measurements were only performed twice. Nevertheless, within the limits of the data a general trend can be postulated. In the first few seconds after injection (between $30 \mathrm{~s}$ and $60 \mathrm{~s}$ ), the intensity increase is minimal and some irregularities are visible. Here, the before mentioned correction is implemented. Another contributor to the irregular shape in the 
beginning is most likely the fluorescence of the free cage which is introduced as a side product at the $30 \mathrm{~s}$ mark. As the method is very sensitive, a sudden injection of a new photo active group disturbs the measurement. After that, the curve shape follows the expected criteria. As initially discussed, multiple explanations for the lower fusion efficiency are possible. First, uncaging was not completed in the $60 \mathrm{~s}$ of laser irradiation. The irradiation time was chosen according to previous uncaging experiments that showed full uncaging after $1 \mathrm{~min}$ in a E3/K3 coiled coil system. ${ }^{[219]}$ Longer uncaging times are not desirable as unexpected side reactions might occur due to the high energy. Furthermore, other experiments in which photo sensitive molecules are present (e.g. FRET quenching assays) would suffer from high photobleaching, rendering the caging group not useful. Therefore, a balance between laser irradiation time and amount of uncaging was chosen. The teal curve shows the outcome of the same experiment except that the K3-Sx vesicles are not treated with light prior to injection. Ideally, the fully caged peptide is introduced into the cuvette and should not show fusion. In comparison to the curve of the lasered experiments, a clear differentiation is possible. The kinetics vary as the slope of the not lasered curve flattens much more quickly. Furthermore, after about $1000 \mathrm{~s}$ the difference between the two in the total intensity becomes more apparent. However, considering the error margins of both curves, the deviation is not as pronounced as expected. Alongside the fusion experiments DLS data was also acquired for each measurement (Table 6.3).

Table 6.3: Sizes of vesicle species used in the uncaging experiments obtained via DLS.

$$
\text { E3-Syb(RKY) K3-Sx(QSXARRX) Mix }
$$

\begin{tabular}{lcccccc} 
Experiment & $\begin{array}{c}\text { Z-Average } \\
\boldsymbol{d}_{\mathbf{h}} / \mathbf{n m}\end{array}$ & PdI & $\begin{array}{c}\text { Z-Average } \\
\boldsymbol{d}_{\mathbf{h}} / \mathbf{n m}\end{array}$ & PdI & $\begin{array}{c}\text { Z-Average } \\
\boldsymbol{d}_{\mathbf{h}} / \mathbf{n m}\end{array}$ & PdI \\
\hline No Laser & 122.3 & 0.082 & 130.3 & 0.070 & 139.9 & 0.117 \\
60 s Laser & 123.4 & 0.076 & 138.5 & 0.120 & 156.9 & 0.136 \\
Before Laser & - & - & 134.2 & 0.083 & - & - \\
\hline
\end{tabular}

The sizes of the E3-Syb vesicles do not differ between the experiments and are of proper quality. For the laser experiment, the sizes of the K3-Sx vesicles were measured before and after irradiation to see whether this influences the size. Indeed, a small increase of $\sim 4 \mathrm{~nm}$ was observed. Additionally, the size distribution broadened significantly as indicated by the PdI values. Apparently, the uncaging process initiated changes in the structure of the vesicles. One possible explanation is that the K3-Sx peptide is more flexible after the 
cleavage of the two bulky caging groups. The enhanced flexibility could lead to an improved interaction of the motif with the own membrane and consequently destabilizing it. This argument can be supported by the result of the control measurement in Figure 6.9. Typically, an increase of $\sim 2 \%$ is recorded for the control measurements. Here, virtually no increase was observed. This phenomenon is explained in more detail in section 4.3.4. For both experiments the DLS data show a size increase after mixing of the liposomes. For the lasered experiment the size increase is twice as high compared to the not lasered mixed samples. This is also accompanied by a larger PdI, which is most likely due to the broader size distribution of the K3-Sx vesicle.

In summary, the introduction of two photocages into the linker region of the K3-Sx peptide was successful. The positioning of the cage at Lys260/264 of syntaxin 1A hampers fusion of the E3/K3-TMD model system. However, it does not completely stop the process. Reasons for this result are manyfold, ranging from pre cleavage of the photocage to insufficient obstruction of the coiled coil formation. Furthermore, the uncaging did not restore full fusion efficiency of the reference system. Nevertheless, the caging of the E3/K3-TMD model system shows potential concerning the elucidation of the fusion mechanism. Further investigations could yield valuable information in this regard. 



\section{Lipids and the E3/K3-TMD System}

Many proteins involved in neuronal exocytosis show interactions with lipids of different nature. For example, synaptotagmin and syntaxin $1 \mathrm{~A}$ are known to interact with $\mathrm{PIP}_{2}$ due to its charge. ${ }^{[257,258]}$ Furthermore, membrane lipid composition is an important factor in liposome fusion and some lipids have been identified to be critical for this activity. ${ }^{[32,259-}$ ${ }^{261]}$ Thus, investigations concerning membrane composition accommodate a high potential for gathering information about the E3/K3-TMD model system. In this chapter the effect of different lipid compositions (section 7.1) on fusion behavior of the E3/K3-TMD model system is presented. The chapters 7.2 and 7.3 show the influence of additional charges in the membrane on the model system.

\subsection{Effect of the Lipid Composition on the E3/K3- TMD System}

In the majority of the fusion experiments in this work, a standard membrane lipid composition of DOPC/DOPE/Cholesterol $=2: 1: 1 \mathrm{~mol} \%$ was used. This composition was used to produce easily comparable results to previous works. ${ }^{[184,185]}$ Furthermore, the netcharge of the membrane is kept mostly neutral (with the exception of the FRET fluorophores) to minimize interactions with the introduced peptides. Still, PC, PE and especially cholesterol concentrations might have an effect on the model system peptides fusion performance. Membrane thickness plays an important part in the TMD's insertion depth and angle. These elements can potentially influence the peptides fusion ability. To investigate whether this is the case, FRET fusion assays with varying membrane lipid compositions have been performed (Figure 7.1). 


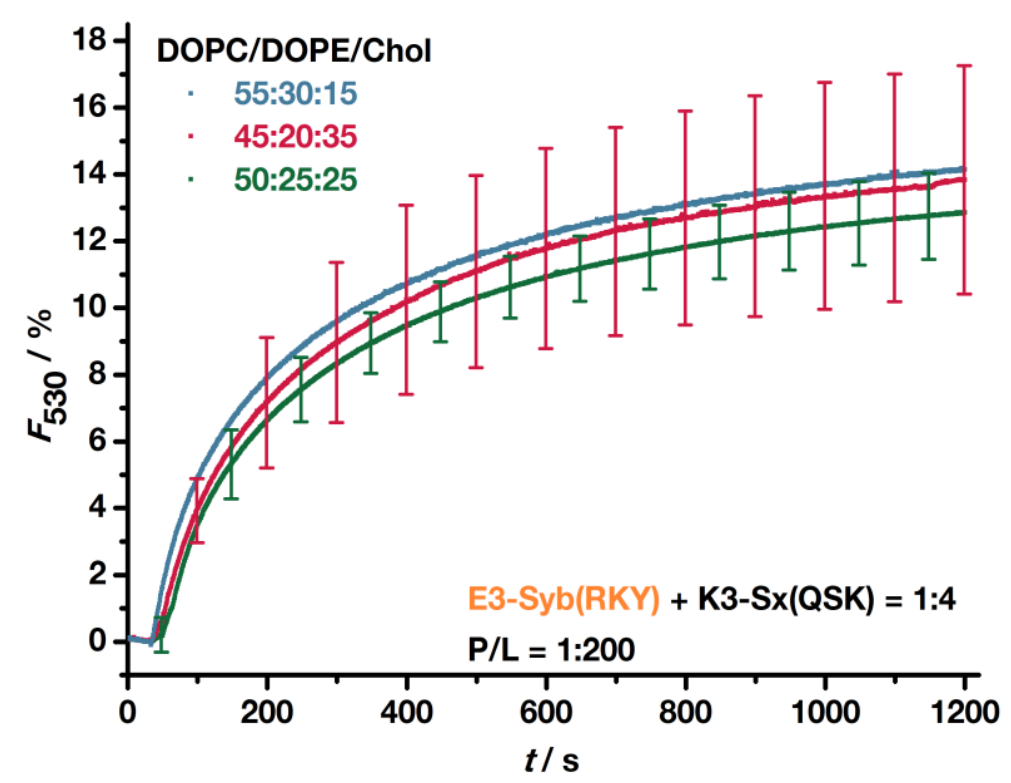

Figure 7.1: FRET dequenching assay with the reference peptides E3-Syb(RKY) and K3Sx(QSK) in varying membrane lipid compositions. (blue) DOPC/DOPE/Chol = 55:30:15. (green) Standard lipid composition DOPC/DOPE/Chol $=2: 1: 1$. (red) DOPC/DOPE/Chol = 45:20:35.

The standard composition of DOPC/DOPE/Chol = 2:1:1 (green curve) shows the typical curve form and a minor standard error. In case of the red curve which depicts a higher cholesterol fraction, a small increase in total fluorescence intensity after $20 \mathrm{~min}$ was observed. Though, this minimal increase is accompanied by a noticeably bigger standard deviation. The increase in cholesterol or the decrease of DOPC and DOPE seem to influence the fusion process in such a way, that the fusion behavior is less consistent. The green curve shows the opposite case with a lower cholesterol fraction in the membrane composition with only $15 \mathrm{~mol} \%$. This measurement was performed only once, therefore the validity is much lower. Nevertheless, it shows a tendency of the effect on fusion efficiency. Previous studies show, that the amount of cholesterol in the membrane has an impact on fusion performance of the native SNARE complex. ${ }^{[262,263]}$ This seems not to be the case for the model system, as small changes in the lipid composition between DOPC, DOPE and cholesterol do not alter the fusion behavior significantly. These results are useful regarding lipid film preparation (see section 9.4.1). Minor inconsistencies, which are unavoidable during the transfer of the lipid solutions, are less significant in case of alterations in the lipid composition. However, changes in the total amount of lipids transferred still have an effect on the number of vesicles in the sample. All in all, small changes in the lipid composition of DOPC, DOPE and cholesterol appear to have no effect on the model systems fusion capabilities. 


\subsection{Incorporation of DOPS in the Vesicle Membrane}

To mimic the natural lipid composition of vesicles in the neuronal exocytosis, experiments with an additional lipid were performed. 1,2-dioleoyl-sn-glycero-3-phospho-L-serine (DOPS) was added in a ratio of DOPC/DOPE/DOPS/Chol $=5: 2: 2: 1$ to reflect the native composition of neuronal vesicles more closely. ${ }^{[264]}$ The results of the lipid mixing assay are shown in Figure 7.2.

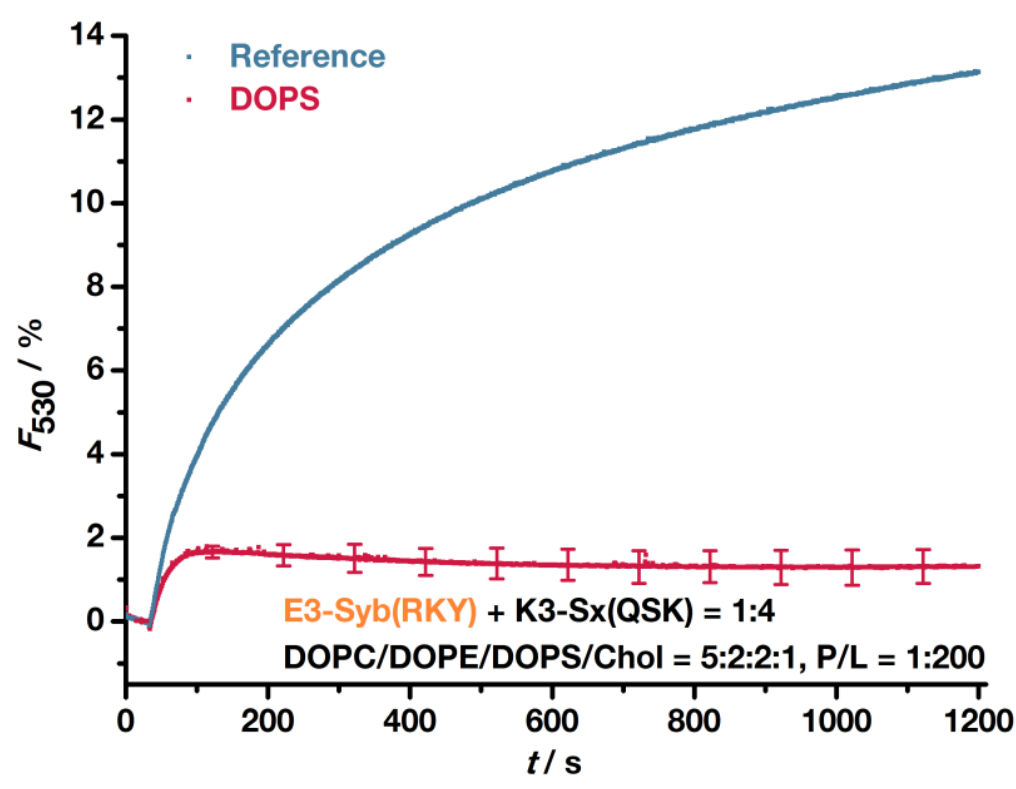

Figure 7.2: FRET dequenching assay of vesicles containing DOPS. (blue) Reference measurement with the standard lipid composition of DOPC/DOPE/Chol $=2: 1: 1$. (red) measurements containing DOPS with DOPC/DOPE/DOPS/Chol = 5:2:2:1.

The results indicate that the E3/K3-TMD system is not able to perform fusion with liposomes containing the above mentioned lipid composition. The measurements show a total fusion efficiency below $2 \%$ after $20 \mathrm{~min}$. The curve form resembles typical control measurements. It has to be noted, that these experiments were performed with a peptide to lipid ratio of 1:200. However, as described in section 4.3.2, the obtained signal in these assays is higher than with a $\mathrm{P} / \mathrm{L}=1: 500$. Therefore, a bigger effect should be observed. Despite this signal enhancing effect, the peptides are apparently not able to perform fusion. DOPS is a negatively charged lipid, and thus changes the electrostatic potential of the vesicles. As DOPS was introduced in both vesicle species, electrostatic repulsion between the vesicles increases. The E3/K3-TMD model system seems to be incapable of 
compensating this phenomenon, hence no fusion can be observed. These results are in agreement with previous findings, showing the inhibitory effect of DOPS in liposome fusion. ${ }^{[265,266]}$ Furthermore, similar results were obtained with the alternative PNA-TMD and Alanyl-PNA-TMD model systems. ${ }^{[188]}$ Another explanation for the dramatic drop in fusion efficiency could be attributed to the motifs used in this system. In contrast to the Alanyl-PNA-TMD and PNA-TMD model systems, the E3/K3-TMD system is highly positively charged. An additional charge inside the membrane, which is introduced by DOPS, can interact with the motifs, immobilizing the peptide on the vesicle membrane. Especially K3-Sx with its highly positive K3-motif might interact more with the lipid head group of DOPS. As mentioned in section 2.7.3, in the E3/K3-PEG-Lipid system, it was demonstrated that $\mathrm{K} 3$ rests on its own membrane until being picked up by $\mathrm{E} 3$ to facilitate fusion. ${ }^{[197,199,203]}$ Introduction of a negatively charged lipid will most likely enhance this behavior. In addition, the linker of syntaxin 1A is positively charged and well known to sequester negatively charged lipids like DOPS or PIP2. ${ }^{[26,241]}$ An additional effect of the recruitment of these lipids is the disassembly of the syntaxin clusters. If K3-Sx clusters form, which has yet to be determined, the addition of negatively charged lipids will probably influence this structure. These results are supported by the data obtained from the DLS measurements with the modified lipid composition as depicted in Table 7.1.

Table 7.1: DLS data of vesicle species with a lipid composition of DOPC/DOPE/DOPS/Chol = 5:2:2:1.

\begin{tabular}{cccccc}
\multicolumn{2}{c}{ E3-Syb(RKY) } & \multicolumn{2}{c}{ K3-Sx(QSK) } & \multicolumn{2}{c}{ Mix } \\
$\begin{array}{c}\text { Z-average } \\
\boldsymbol{d}_{\mathbf{h}} / \mathbf{n m}\end{array}$ & PdI & $\begin{array}{c}\text { Z-average } \\
\boldsymbol{d}_{\mathbf{h}} / \mathbf{n m}\end{array}$ & PdI & $\begin{array}{c}\text { Z-average } \\
\boldsymbol{d}_{\mathbf{h}} / \mathbf{n m}\end{array}$ & PdI \\
\hline 137.5 & 0.096 & 137.8 & 0.091 & 141.0 & 0.120 \\
145.1 & 0.089 & 136.1 & 0.090 & 140.2 & 0.117 \\
\hline
\end{tabular}

The size difference of the K3-Sx vesicle and E3-Syb vesicles is typically $\sim 10 \mathrm{~nm}$ with the K3-Sx vesicles being bigger for the standard lipid composition of DOPC/DOPE/Chol $=2: 1: 1$. Here, in both measurements the size is either the same or even smaller than the E3-Syb-vesicles. Evidently, a change in the vesicles is already visible before the fusion assays are performed, which leads to the conclusion, that interactions inside the vesicle membrane have changed. The mixed vesicle population post FRET dequenching assay shows no significant increase in size. Consequently, the initial vesicle species did not fuse, backing the results obtained from the fusion assay. 
Ultimately, DOPS inhibts fusion activity of the E3/K3-TMD complex. This is probably due to the introduced charges in the liposomes. Electrostatic interactions between DOPS and the peptides, as well as repulsion between the negatively charged vesicles might be the crucial factor for this result.

\subsection{Effect of $\mathrm{PIP}_{2}$ on Membrane Fusion}

The interaction between phosphatidylinositol 4,5-bisphosphate ( $\left.\mathrm{PIP}_{2}\right)$ and syntaxin $1 \mathrm{~A}$ has been a research topic for many years. As discussed in section 7.2, negatively charged lipids interact with positively charged areas of SNARE proteins. The polybasic area (KARRKK) in syntaxin's linker has previously shown to be a major partner for these interactions. ${ }^{[28,172,241,267]}$ Given the fact, that the K3-Sx model peptide includes the polybasic area, experiments have been performed with different $\mathrm{PIP}_{2}$ concentrations to investigate the behavior of the model system (Figure 7.3).

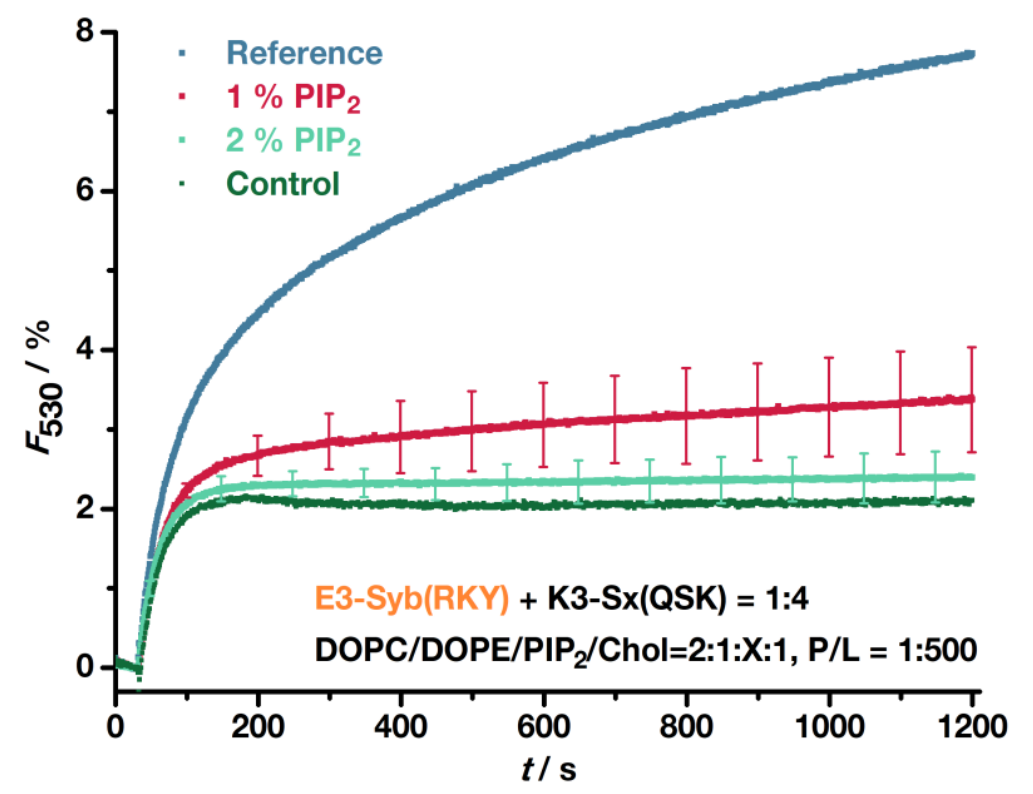

Figure 7.3: Fusion curves obtained in FRET dequenching assays with different amounts of $\mathrm{PIP}_{2}$. (blue) Reference measurement containing no $\mathrm{PIP}_{2}$. (red) Measurements with $1 \% \mathrm{PIP}_{2}$. (teal) Measurements containing $2 \% \mathrm{PIP}_{2}$. (green) Control measurement with $\mathrm{K} 3-\mathrm{Sx}(\mathrm{QSK})$ and $2 \% \mathrm{PIP}_{2}$.

A range between $0.01-2$ mol\% $\mathrm{PIP}_{2}$ has been chosen for the fusion assays. The initial measurements were done with $2 \% \mathrm{PIP}_{2}$ and showed, that this amount is sufficient to stop the model system from being able to fuse vesicles. The control measurement also included 
$2 \% \mathrm{PIP}_{2}$ in the $\mathrm{K} 3-\mathrm{Sx}(\mathrm{QSK})$ vesicle as well as in the labeled vesicle. The difference between both is insignificantly small, also including the standard deviation of the measurements. The measurements with $1 \% \mathrm{PIP}_{2}$ are slightly higher in intensity but are also accompanied with a bigger error. Nevertheless, a trend is visible with these two PIP $_{2}$ concentrations. Lower amounts of $\mathrm{PIP}_{2}$ seem to restore the ability of the model system to perform fusion. To verify this trend measurements with $0.1 \%$ and $0.01 \% \mathrm{PIP}_{2}$ were conducted as depicted in Figure 7.4.

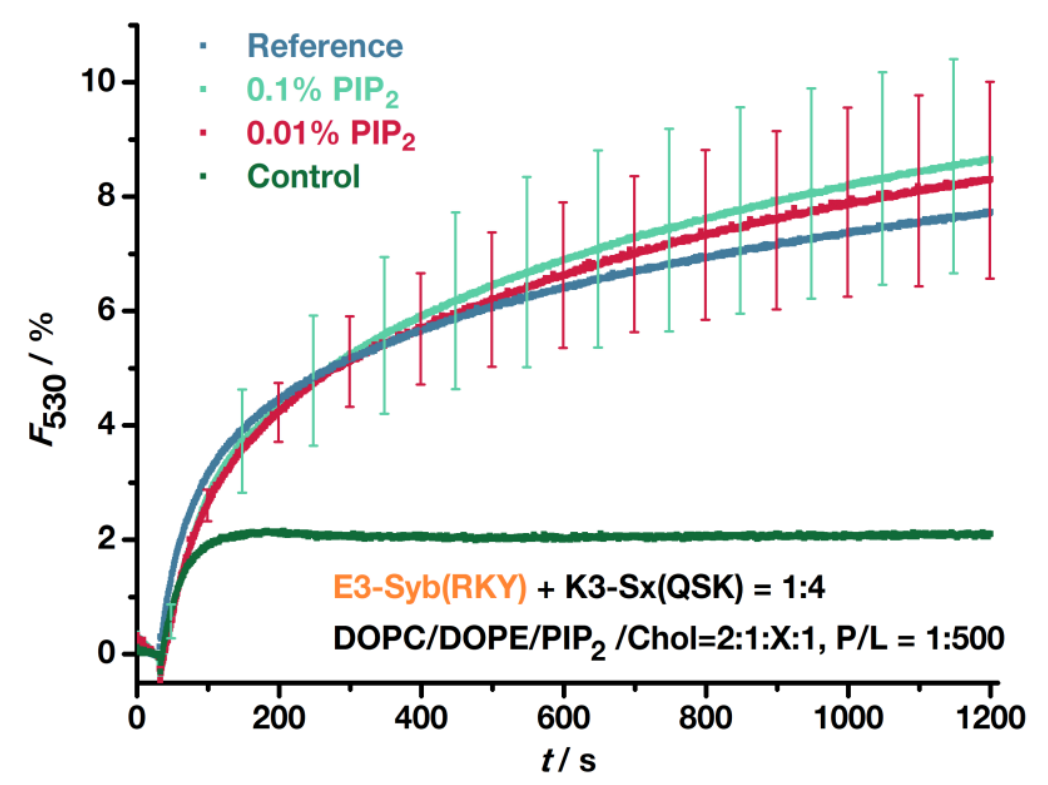

Figure 7.4: Fusion curves obtained in FRET dequenching assays with different amounts of $\mathrm{PIP}_{2}$. (blue) Reference measurement containing no $\mathrm{PIP}_{2}$. (teal) Average of measurements containing $0.1 \% \mathrm{PIP}_{2}$. (red) Measurements with $0.01 \% \mathrm{PIP}_{2}$. (green) Control measurement with $\mathrm{K} 3-\mathrm{Sx}(\mathrm{QSK})$ and $2 \% \mathrm{PIP}_{2}$.

For both $\mathrm{PIP}_{2}$ concentrations, $0.1 \%$ and $0.01 \%$, a similar result was obtained. The curve shape is as expected for this kind of experiment. The reference measurement does not contain $\mathrm{PIP}_{2}$ in the vesicles and resembles therefore the final measurement of the concentration gradient. The standard deviation of the $0.1 \%$ and $0.01 \% \mathrm{PIP}_{2}$ vesicle species is equally big, rendering both sets of measurements virtually indistinguishable from each other.

Literature suggests, that a concentration of $\sim 6 \mathrm{~mol} \%$ of $\mathrm{PIP}_{2}$ inhibits SNARE-dependent liposome fusion. ${ }^{[28]}$ Here, as low as 1 mol\% was sufficient to inhibit the model systems ability to fuse vesicles. Possible explanations are similar to the ones in section 7.2. A similarity between DOPS and $\mathrm{PIP}_{2}$ are the negatively charged headgroups. In fact, depending on the $\mathrm{pH}, \mathrm{PIP}_{2}$ can adopt charges ranging from $(-3)$ to $(-5) \cdot{ }^{[268]}$ This makes it 
an even better binding partner for the polybasic stretch of amino acids in K3-Sx peptides. This is also true for the lysines of the K3 motif. K3-Sx is probably firmly attached to the vesicle membrane, making it unavailable for E3-Syb in the opposing vesicle and consequently inhibiting fusion. Taking into account the total number of $\mathrm{PIP}_{2}$ and putting it into perspective of the number of peptides present, an explanation on the molecular level is possible. The peptide to lipid ratio in these experiments was $\mathrm{P} / \mathrm{L}=1 / 500$. With a concentration of $1 \mathrm{~mol} \%$, about five of these lipids are $\mathrm{PIP}_{2}$ lipids. This means, that on average every peptide can be accompanied by five $\mathrm{PIP}_{2}$ lipids, which might interact with the basic linker or motif. If the amount of $\mathrm{PIP}_{2}$ is dropped to $0.1 \mathrm{~mol} \%$, only 0.5 lipids per peptide are available in the vesicle. This is not sufficient to stop the vesicle in its entirety to fuse. Furthermore, it is highly likely that the $\mathrm{PIP}_{2}$ lipids are being recruited by K3-Sx, as this is the case for the natural syntaxin $1 \mathrm{~A}$ protein due to the polybasic stretch of amino acids in the linker. ${ }^{[28,269]}$ The acquired DLS data of the different measurements is presented in Table 7.2.

Table 7.2: Average of the recorded sizes of vesicle species used with varying PIP $_{2}$ concentrations.Data obtained via DLS.

\begin{tabular}{ccccccc} 
& \multicolumn{2}{c}{ E3-Syb(RKY) } & \multicolumn{2}{c}{ K3-Sx(QSK) } & \multicolumn{2}{c}{ Mix } \\
PIP2 & $\begin{array}{c}\text { Z-average } \\
\boldsymbol{d}_{\mathbf{h}} / \mathbf{n m}\end{array}$ & PdI & $\begin{array}{c}\text { Z-average } \\
\boldsymbol{d}_{\mathbf{h}} / \mathbf{n m}\end{array}$ & PdI & $\begin{array}{c}\text { Z-average } \\
\boldsymbol{d h} / \mathbf{n m}\end{array}$ & PdI \\
\hline $0.00 \mathrm{~mol} \%$ & 128.5 & 0.091 & 131.7 & 0.102 & 149.9 & 0.131 \\
$0.01 \mathrm{~mol} \%$ & 120.2 & 0.084 & 133.3 & 0.106 & 151.9 & 0.123 \\
$0.10 \mathrm{~mol} \%$ & 128.4 & 0.090 & 141.2 & 0.114 & 161.7 & 0.153 \\
$1.00 \mathrm{~mol} \%$ & 117.3 & 0.076 & 127.9 & 0.085 & 134.9 & 0.110 \\
$2.00 \mathrm{~mol} \%$ & 133.4 & 0.087 & 147.0 & 0.088 & 147.1 & 0.097 \\
\hline
\end{tabular}

The observations made from the FRET dequenching assays are also supported by the DLS data. PIP 2 concentrations below $1 \mathrm{~mol} \%$ show a significant increase in the average size of the mixed vesicle population post fusion. For the $1 \mathrm{~mol} \% \mathrm{PIP}_{2}$ concentration measurements, only a small size increase was recorded.

In combination with the dequenching assay data, it can be concluded that the 1 mol\% mark is on the edge of the inhibitory effect of the lipid. The 2 mol\% measurements clearly show that no size increase occurred confirming the inhibition due to $\mathrm{PIP}_{2}$. 



\section{Conclusion and Outlook}

This thesis focused on the linkers of the SNARE mimicking E3/K3-TMD model system. The system consists of the artificial E3/K3 coiled coil and the native sequences of the linker and TMD of synaptobrevin 2 and syntaxin 1A to form the E3-Syb and K3-Sx peptides. Modifications on the linkers of both peptides were performed and the changes in the fusion behavior in FRET dequenching assays recorded. Additionally, changes in the vesicle sizes due to the incorporated modified peptides were monitored with DLS. Insight into the fusion mechanism was gained by the introduction of photocleavable groups into one of the peptides. Furthermore, the effect of different lipid compositions and the introduction of charged lipids on the fusion behavior of the system was examined.

To gather reliable data and produce more consistent results, peptide quality and measurement parameters have been optimized. Reaction conditions for the microwave assisted automated peptide synthesis were specifically tailored for the used peptides. This approach lead to less truncated peptides and to an overall increase in peptide quality. Peptide purification via HPLC improved the purity even further with considerable changes to the model systems fusion behavior. The unexpected reduction in fusion efficiency with purified peptides, emphasized the importance of these two steps.

An improvement in the quality of the collected data during FRET dequenching assays and DLS measurements was achieved by changing the peptide to lipid ratio in the vesicles to $\mathrm{P} / \mathrm{L}=1: 500$. The lower overall signal intensity during the fusion assays was compensated by substantial improvements in the mean variation between measurements of the same type. The smaller error bars and consequently more convincing data was gained due to the improved vesicle quality. Notably, the improvements in the K3-Sx vesicle stability lead to these results. In previous experiments with this system, the stability of the vesicles was the limiting factor during fusion assays. ${ }^{[207]}$ The improvements introduced in this work are therefore expanding the potential of the E3/K3-TMD system. 
During the optimizations of the different experiments, first clues regarding the model systems fusion behavior could be obtained. It is possible, that K3-Sx peptides interact with the FRET fluorophores on the opposing vesicle membrane. The same behavior was also found in the similar PNA-TMD model system. ${ }^{[188]}$ Furthermore, this observation is in line with experiments regarding the lipid composition of the vesicles. The addition of charged lipids e.g. DOPS or PIP $_{2}$ into the vesicle membranes lead to a reduced or even completely abolished fusion efficiency of the system. One possible explanation for these observations is that the K3-Sx peptide interacts strongly with the own membrane due to the negative charges. The motif would not be available for the coiled coil formation with E3-Syb. Consequently, no fusion can be detected. Interaction of the $\mathrm{K} 3$ motif with the membrane was already investigated with the E3/K3-PEG-lipid system. ${ }^{[200,203]}$ Due to the similarities between the two SNARE analoga, it is plausible that this behavior is also exhibited in the E3/K3-TMD system used in this thesis.

However, to verify this theory, more tests remain to be performed. For example, the use of two vesicle populations with different lipid compositions could yield more information regarding this question. If the $\mathrm{K} 3$ motif is attached to the own vesicle membrane due to charged lipids, no fusion should be detected with liposome species lacking these lipids. The other way round, fusion is expected if the opposing vesicle is the one containing the negatively charged lipids. The positively charged K3 motif should prefer interaction with the other membrane. This experiment would be similar to the ones performed in section 4.3.3. Another approach is to measure the distance of the $\mathrm{K} 3$ motif to the membrane. This can be achieved via tryptophan fluorescence emission, which is sensitive to the polarity of the environment. ${ }^{[270,271]}$

The incorporation of the photocleavable protecting group into the K3-Sx peptide impaired the ability of the system to perform fusion. The jump in fluorescence intensity which was detected after the caged peptide was exposed to light irradiation confirms the intended effect of the group. Yet, this experiment is afflicted with several difficulties. Photobleaching of the FRET fluorophores due to the laser had to be accounted for. Also, the side products of the cleaved group interfered with the fluorescence measurements. These findings lead to adaptions of the experiment, where photobleaching due to the laser was removed as a factor. During these new measurements, the vesicles containing the caged peptides were separately lasered and subsequently introduced to the labeled vesicles. In comparison to the fully caged species, a difference in fusion behavior was observed. 
However, the inhibition was not absolute, as the measurements with the fully caged K3-Sx peptide still showed some positive fusion activity. Due to the difficult synthesis and handling of the peptide, a small percentage of the protecting groups was already missing before the fusion assays were started. Consequently, some peptides were not sterically hindered and could fuse without problems. Furthermore, the positioning of the two cages might not be hindering the interaction between the two peptides sufficiently to stop the fusion completely.

To improve this experiment, the inhibiting effect of the group could be enhanced by changing the positions in the peptide sequence or even introduce one additional group. A promising candidate would be Lys 265 of syntaxin due to its orientation in the cis-SNARE complex.

In this thesis, the connection between the artificial motif and natural linker sequence was investigated. Analysis of the three dimensional conformation of the natural SNARE ciscomplex and the fundamentals of coiled coil heptad repeat interactions suggested that the original peptide sequence of the E3/K3-TMD model system was not optimal regarding intermolecular interactions between the peptides. To investigate this, a peptide pair with modified length was synthesized and its fusion behavior monitored. These modifications lead to a slightly reduced fusion efficiency compared to the reference system. Apparently, the model systems conformation and fusion behavior differ compared to the natural SNARE machinery. Accordingly, the initial design seems to be the best performing variant of this SNARE analog.

Investigations concerning the aromatic collar in the linkers of synaptobrevin and syntaxin showed that they are important to maintain a high fusion efficiency of the system. However, in comparison to the natural machinery, opposing results were obtained in respect to the number of substituted residues. Whereas merely the substitution of both Trp 89/90 in synaptobrevin showed a change in the exocytotic burst of chromaffin cells, ${ }^{[29]}$ the exchange of already one of the tryptophan residues decreased the fusion behavior of the E3/K3-TMD complex. Substitution of Y257A in the K3-Sx peptide and Y88A in the E3-Syb species did not lead to conclusive results. The mean variation between the individual measurements was significantly higher compared to other fusion assays in this work. In addition to the substituted amino acids, K3-Sx had to be elongated by at least one amino acid to include the tyrosine residue, as it is not included in the original sequence. Ultimately, two amino 
acids were added to the peptide. This approach entailed the need for an elongated reference peptide without the tyrosine substitution. The comparison between elongated reference and the tyrosine substituted assays suggested an inhibitory effect of the tyrosines. Considering the high error margin of the measurements, further tests have to be conducted before a more accurate conclusion can be made.

The polybasic area in syntaxin is a center of research interest, as interactions with a variety of lipids and other proteins have been observed in the past. ${ }^{[26,28,172]}$ To answer the question whether this is also true for the E3/K3-TMD system, specific amino acids have been exchanged. The substitution of Lys 260/264 in K3-Sx with alanine reduced the fusion efficiency by $\sim 40 \%$ compared to the reference system. Initially, these lysines have been chosen due to their position sitting in between synaptobrevin 2 and syntaxin $1 \mathrm{~A} .{ }^{[23]}$ Thus, possible stabilizing interactions would be eliminated and more information about their magnitude during the fusion mechanism could be gathered. An even bigger effect was observed during experiments where the two Arg 262/263 of K3-Sx were substituted for alanine. Fusion could not be observed during the FRET dequenching assays. Due to the arginines being adjacent to the two Lys 260/264 and having the same charge, the orientation in the peptide is most likely the reason for this result. In the natural SNARE cis-complex, the arginines point toward the membrane, which enables them to interact with lipids or other proteins. However, as the exact conformation of the model system is not identified, additional experiments have to be executed to verify this hypothesis.

Although, the E3/K3-TMD system was verified to perform full fusion via content mixing assays in the past, ${ }^{[22,206]}$ due to the many improvements in peptide and vesicle quality, these experiments have to be repeated under the new conditions. Additionally, inner lipid mixing assays as described by HUBRICH need to be executed with this system as well to distinguish between hemi- and full fusion. ${ }^{[185]}$ Further insight about the fusion behavior could also be obtained with FCCS measurements or using pore-spanning membranes to record single fusion events. ${ }^{[272,273]}$ 


\section{Material and Methods}

\subsection{Solvents and Reagents}

All used solvents were of laboratory grade $(\geq 99.8 \%)$ or higher. Ultrapure water was obtained by filtering demineralized water with an arium ${ }^{\circledR}$ mini ultrapure water system from Sartorius (Göttingen, Germany). Solvents for HPLC and UPLC were of HPLC grade quality (99.9\%). Solvents used for LC-MS were of LC-MS grade quality. Commercially available reagents were purchased from Merck KGaA (Darmstadt, Germany), SigmaAldrich GmbH (München, Germany) and Fisher Scientific GmbH (Schwerte, Germany). Amino acids were purchased from GL Biochem (Shanghai) Ltd. (Shanghai, China) and Sigma-Aldrich GmbH (München, Germany). Lipids and fluorophores were purchased from Avanti Polar Lipids, Inc. (Alabaster, USA). Fluorinated solvents were obtained from chemPUR (Karlruhe, Germany). Phospholipids were purchased from Avanti Polar Lipids, Inc. (Alabaster, USA).

\subsection{General Methods}

\subsubsection{Lyophilization}

Aqueous samples were thoroughly frozen in liquid nitrogen and dried in vacuo $(<1$ mbar $)$ using a Alpha 2-4 LD freeze dryer form Christ (Osterode am Harz, Germany) which was connected to a high vacuum pump. Raw peptides were solved in small amounts of HFIP and treated in a ultrasonic bath. The solution was diluted with a tenfold amount of ultrapure water and prior to freezing stirred thoroughly. Fractions collected from HPLC purification were reduced via nitrogen stream to a volume $\sim 2 \mathrm{~mL}$ and diluted with $6 \mathrm{~mL}$ ultrapure water before freezing. 


\subsubsection{Storage}

Freeze dried peptides and peptides solved in TFE were stored at $-18{ }^{\circ} \mathrm{C}$ in plastic tubes. Peptides attached to resins were stored under vacuum at room temperature. Chemicals were stored according to suppliers recommendation. Lipids were diluted in chloroform and stored under argon atmosphere in glass bottles at $-18{ }^{\circ} \mathrm{C}$. HEPES buffer stock solution (10x) was stored at $8{ }^{\circ} \mathrm{C}$. Light sensitive compounds were additionally protected from light.

\subsubsection{Mass Spectrometry}

ESI (Electrospray ionization) mass spectra were recorded using a maXis spectrometer by Bruker Daltonik GmbH (Bremen, Germany) with a TOF analyser. The peptides were dissolved in HFIP and mixed with $\mathrm{MeOH}$ in a 1:10 ratio. The mass-to-charge ratio $(\mathrm{m} / \mathrm{z})$ with the corresponding relative intensity is reported.

\subsubsection{LC-MS}

LC-MS measurements were done on the UltiMate 3000 from Thermo Fischer Scientific (Waltham, USA) consisting of pumps, autosampler, column oven and diode array detector of the 3000 series. The UPLC was connected to the ion trap mass spectrometer LTQ XL from Thermo Fischer Scientific (Waltham, USA). The mobile phase used water (LC-MS grade $)+0.1 \%$ FA and MeCN $($ LC-MS grade $)+0.1 \%$ FA. The column used for separation was an ACE Excel (100A-2-C18 $100 \mathrm{~mm}$ x $2.1 \mathrm{~mm}, 2 \mu \mathrm{m})$ which operated under a flow rate of $0.35 \mathrm{~mL} / \mathrm{min}$ at $40^{\circ} \mathrm{C}$. Detection was done at $215 \mathrm{~nm}, 254 \mathrm{~nm}, 280 \mathrm{~nm}$ and $380 \mathrm{~nm}$.

\subsubsection{Nuclear Magnetic Resonance Spectroscopy}

Measurements of ${ }^{1} \mathrm{H}$ - and ${ }^{13} \mathrm{C}-\mathrm{NMR}$ were recorded on a Mercury-VX 300 or VNMRS-300 from Varian (Palo Alto, USA). The deuterated solvents and measurement frequencies are disclosed at the respective compounds. As the internal standard, the residual proton signal of the solvent was used. The chemical shift $\delta$ is given in ppm and coupling constants $J$ in Hz. Coupling between nuclei is denoted with the following abbreviations: s (singlet), $\mathrm{d}$ (doublet), $\mathrm{t}$ (triplet), q (quartet) or m (multiplet). 


\subsubsection{UV/Vis Spectroscopy}

UV/Vis spectroscopy was used to determine the phosphorus content (see section 9.4.6) and the peptide concentrations of samples. For both, either NanoDrop 2000c or NanoDrop OneC from Thermo Fischer Scientific (Waltham, Massachusetts, USA) was used. As cuvettes a quartz SUPRASIL QS or disposable cuvettes from Brand GmbH (Wertheim, Germany) with a path length of $10 \mathrm{~mm}$ were used. Prior to the measurements a blank was measured with $195 \mu \mathrm{L}$ of MeCN (HPLC grade) and $5 \mu \mathrm{L}$ of TFE. The corresponding value was automatically subtracted by the software from the following measurements. The peptide concentration was determined at $280 \mathrm{~nm}$ or $385 \mathrm{~nm}$ for photocaged peptides with the Beer-Lambert law following equation 9.1.

$$
c=\frac{A}{\varepsilon \cdot d}
$$

The absorption $A$ is measured and corrected by the blank measurement, the molar extinction coefficient $\varepsilon$ is specific for every peptide and the path distance $d$ is determined by the cuvette which is usually $10 \mathrm{~mm}$. The extinction coefficient was calculated for every peptide individually by summation of every amino acids or building blocks contribution at the measured wavelength. The coefficients used in this thesis are listed in Table 9.1.

Table 9.1: Extinction coefficients used in this thesis.

\begin{tabular}{lccc} 
Fluorophore & $\begin{array}{c}\text { Absorption } \boldsymbol{\lambda} \\
\mathbf{n m}\end{array}$ & $\begin{array}{c}\text { Extinction coefficient } \boldsymbol{\varepsilon} \\
\mathbf{M}^{\mathbf{- 1}} \cdot \mathbf{c m}^{-\mathbf{1}}\end{array}$ & Reference \\
\hline Cysteine & 280 & 120 & {$[274]$} \\
Tryptophan & 280 & 5690 & {$[274]$} \\
Tyrosine & 280 & 1280 & {$[274]$} \\
DEACM & 385 & 16000 & {$[256]$} \\
\hline
\end{tabular}

\subsubsection{Purification via RP-HPLC}

Analysis and bulk purification of all peptides was done via reversed phase high performance liquid chromatography (RP-HPLC) using the HPLC systems listed in Table 9.2 . 
Table 9.2: HPLC-Systems used for analysis and bulk purification of peptides.

System

Modification

\begin{tabular}{|c|c|}
\hline UPLC & $\begin{array}{l}\text { Thermo Fisher (Waltham, Massachusetts, USA). } \\
\text { Ultimate 3000: Detector DAD-3000; pumps LPG-3400SD; column oven } \\
\text { TCC-3000SD; autosampler WPS-3000SL; software Chromeleon } 7.2 \text { SR4. }\end{array}$ \\
\hline HPLC Prep & $\begin{array}{l}\text { JASCO (Tokyo, Japan) } \\
\text { Detector UV-4075, pumps PU-4086, column oven CO-4060, interface } \\
\text { LC-NetII/ADC, collector interface Fraction collector interface, collector } \\
\text { CHF 122SC (Advantec), software ChromNav 2.01.06. }\end{array}$ \\
\hline $\begin{array}{c}\text { HPLC } \\
\text { Semi-Prep }\end{array}$ & $\begin{array}{l}\text { JASCO } \text { (Tokyo, Japan) } \\
\text { Detector } 2 \text { x } U V-2075 \text { Plus; } U V-975 \text {, pumps PU-2080 Plus, column oven } \\
\text { CO-4060, interface LC-NetII/ADC, degasser DG-2080-53, collector } \\
\text { interface Fraction collector controller, software ChromNav 2.01.06. }\end{array}$ \\
\hline $\begin{array}{l}\text { HPLC Prep- } \\
\text { DAD }\end{array}$ & $\begin{array}{l}\text { JASCO (Tokyo, Japan) } \\
\text { Detector } M D-2010 \text { Plus, pumps } P U-2080 \text { Plus } 20 \mathrm{ml} \text { version, interface } \\
\text { LC-NetII/ADC, degasser } D G-2080-53 \text {, collector interface } F C \text {-2088-30, } \\
\text { collector } C H F 122 S C \text { (Advantec), software ChromNav 2.01.06. }\end{array}$ \\
\hline
\end{tabular}

For the stationary phase, columns from Advanced Chromatography Technologies Ltd. (Aberdeen, Scotland) listed in Table 9.3 were used.

Table 9.3: Columns used for peptide analysis and purification and the flow rates at which they were used.

\begin{tabular}{clll} 
Column & Name & Specification & Flow rate \\
\hline 1 & ACE Excel 2 C18 & C18,100 x 2.1 mm,100, $2 \mu \mathrm{m}$ & $0.3 \mathrm{~mL} / \mathrm{min}$ \\
2 & ACE Excel 5 C18 & $\mathrm{C} 18,150 \times 10 \mathrm{~mm}, 100 \AA, 5 \mu \mathrm{m}$ & $5.0 \mathrm{~mL} / \mathrm{min}$ \\
3 & ACE Excel 5 C18 & $\mathrm{C} 18,150 \times 21.2 \mathrm{~mm}, 100 \AA, 5 \mu \mathrm{m}$ & $10 \mathrm{~mL} / \mathrm{min}$ \\
\hline
\end{tabular}

As eluents, different gradients of ultrapure water, acetonitrile and methanol as depicted in Table 9.4 were used. Generally, the sample was first solved in HFIP. For every $10 \mathrm{mg}$ of peptide sample $100 \mu \mathrm{L}$ HFIP were used. To accelerate solvation, the sample was treated in a sonication bath until no solid residues were visible to the naked eye. The sample was then diluted with ultrapure water to a maximum volume of $700 \mu \mathrm{L}$. Subsequent, filtration using a Phenex-RC syringe filter ( $0.45 \mu \mathrm{m}$ pore size) from Phenomenex (Aschaffenburg, Germany) was performed. 
Table 9.4: List of solvent systems used during LC peptide analysis and purification.

\begin{tabular}{cll} 
Solvent System & Solvent A & Solvent B \\
\hline I & $\mathrm{H}_{2} \mathrm{O}+0.1 \%$ TFA & $\mathrm{MeOH}+0.1 \%$ TFA \\
II & $\mathrm{H}_{2} \mathrm{O}+0.1 \%$ TFA & $\mathrm{MeCN}+0.1 \%$ TFA \\
III & $\mathrm{H}_{2} \mathrm{O}+0.1 \%$ TFA & $\mathrm{MeCN}+0.85 \%$ TFA \\
\hline
\end{tabular}

Absorbance was detected at $215 \mathrm{~nm}, 280 \mathrm{~nm}$ and optionally at $254 \mathrm{~nm}$. Peptides with fluorophores were detected at $380 \mathrm{~nm}$.

\subsubsection{Flash chromatography}

Silica gel for flash chromatography was obtained by Merck KGaA (Darmstadt, Germany) with a grain size of 40-63 $\mu \mathrm{m}$. The crude sample was eluted in a suitable solvent, mixed with a small amount of silica gel and afterwards separated by the solvent using a rotary evaporator. The column was run at low overpressure.

\subsubsection{Thin layer Chromatography}

Reaction monitoring was performed via thin layer chromatography with aluminum assisted silica gel 60 F254 from Merck KGaA (Darmstadt, Germany). Detection of the sample was achieved by fluorescence deletion at $254 \mathrm{~nm}$.

\subsubsection{Graphs and Illustrations}

Graphs depicting experimental data were plotted with OriginPro 8.5 from OriginLab (Massachusetts, USA), images of structures obtained by X-ray data were edited with the UCSF Chimera package (California, USA), chemical structures were drawn with ChemDraw Professional from PerkinElmer (Massachusetts, USA) and all other illustrations were created using Affinity Designer, Affinity Photo and Affinity Publisher by Serif Inc. (Nottingham, Great Britain). 


\subsection{Peptide Synthesis}

\subsubsection{Solid Phase Peptide Synthesis (SPPS)}

All peptides were synthesized using automated microwave assisted peptide synthesizers. For the synthesis either a Liberty Blue or Liberty Prime peptide synthesizer from CEM (Matthews, North Carolina, USA) was used, both were connected to a Discover microwave unit by $C E M$. The exact configurations are listed in Table 9.6.

Table 9.5: List of synthesizers used for automated microwave assisted peptide synthesis.

\section{Synthesizer Name Configuration}

Liberty Blue 1

Liberty Blue 2
Liberty Blue, Discover microwave, software Liberty Blue Application Software vers. 1.50.

Liberty Blue, Discover microwave, software Liberty Blue Application Software vers. 2.2.

Depending on the synthesizer, different concentrations of reagents were used (see Table 9.6). As solvent $N, N$-Dimethylformamide (DMF), as activator $N, N^{\prime}-$ Diisopropylcarbodiimide (DIC), as activator base Oxyma Pure (Ethyl cyano(hydroxyimino)acetate) and as deprotection piperidine with $N, N$-Diisopropylethylamine (DIPEA) was used.

Table 9.6: Used amino acids and concentrations of reagents for automated peptide synthesis on the Liberty Blue synthesizers.

\begin{tabular}{cc}
\hline Reagent & Concentration \\
\hline Amino acids & $0.2 \mathrm{M}$ \\
DIC & $0.5 \mathrm{M}$ \\
Oxyma & $0.5 \mathrm{M}$ \\
Piperidine & $20 \%$ \\
DIPEA & $0.1 \mathrm{M}$ \\
\hline Fmoc-Ala-OH, Fmoc-Arg(Pbf)-OH, Fmoc-Asn(Trt)-OH, Fmoc-Cys(Trt)-OH, \\
Fmoc-Gln(Trt)-OH, Fmoc-Glu(OtBu)-OH, Fmoc-Gly-OH, Fmoc-Ile-OH, \\
Fmoc-Leu-OH, Fmoc-Lys(Boc)-OH, Fmoc-Met-OH, Fmoc-Phe-OH, Fmoc- \\
Ser(tBu)-OH, Fmoc-Trp(Boc)-OH, Fmoc-Tyr(tBu)-OH, Fmoc-Val-OH \\
\hline
\end{tabular}

For all K3-Sx peptides a preloaded Fmoc-Gly-Wang LL resin with a loading density between $0.33-0.36 \mathrm{mmol} / \mathrm{g}$, for all E3-Syb peptides a preloaded Fmoc-Thr(tBu)-Wang LL 
resin with a loading density of $0.28-0.34 \mathrm{mmol} / \mathrm{g}$ was used. Prior to synthesis, the resin was swollen with DMF for a minimum of $10 \mathrm{~min}$.

Synthesis conditions varied between specific amino acids as well as position in the peptide. All used coupling methods are listed in Table 9.7. Generally, amino acids coupled to the peptide from the position 30 onwards, were coupled twice to ensure higher yields.

Table 9.7: List of the different coupling methods used for peptide synthesis.

\begin{tabular}{|c|c|}
\hline Name & Method \\
\hline Optimized & $\begin{array}{l}1.75^{\circ} \mathrm{C}, 170 \mathrm{~W}, 17 \mathrm{~s} ; 2.90^{\circ} \mathrm{C}, 30 \mathrm{~W}, 225 \mathrm{~s} . \\
5 \text { eq. aa, } 5 \text { eq. DIC, } 5 \text { eq. OxymaPure, } 0.5 \text { eq. DIPEA. }\end{array}$ \\
\hline Carbomax & $\begin{array}{l}1.85^{\circ} \mathrm{C}, 280 \mathrm{~W}, 20 \mathrm{~s} ; 2.105^{\circ} \mathrm{C}, 90 \mathrm{~W}, 75 \mathrm{~s} . \\
5 \text { eq. aa, } 10 \text { eq. DIC, } 5 \text { eq. OxymaPure, } 0.5 \text { eq. DIPEA. }\end{array}$ \\
\hline Arginine & $\begin{array}{l}\text { First coupling: } 1.25^{\circ} \mathrm{C}, 0 \mathrm{~W}, 1500 \mathrm{~s} ; 2.75^{\circ} \mathrm{C}, 30 \mathrm{~W}, 120 \mathrm{~s} \text {. } \\
\text { Second coupling: } 75^{\circ} \mathrm{C}, 30 \mathrm{~W}, 300 \mathrm{~s} .\end{array}$ \\
\hline Cysteine & 1. $25^{\circ} \mathrm{C}, 0 \mathrm{~W}, 120 \mathrm{~s} ; 2.50^{\circ} \mathrm{C}, 30 \mathrm{~W}, 480 \mathrm{~s}$. \\
\hline
\end{tabular}

Generally, the different coupling methods follow the same procedure. First, the N-terminal amino acid on the resin is deprotected using piperidine at elevated temperatures $\left(1.75^{\circ} \mathrm{C}\right.$, $\left.210 \mathrm{~W}, 15 \mathrm{~s} ; 2.90^{\circ} \mathrm{C}, 30 \mathrm{~W}, 50 \mathrm{~s}\right)$. Second, the next amino acid, activator and activator base are added to the resin. Depending on the peptide and specific amino acid a method from Table 9.7 was used. Finally, the resin is washed several times with DMF and the next coupling step is started. After the coupling of the last amino acid, a final Fmoc deprotection was performed and the resin was washed with DMF. The resin was then transferred to a BD syringe equipped with a polyethylene frit and washed three times with DCM. Lastly, the resin was dried for at least 30 min in vacuo.

\subsubsection{Resin cleavage}

Cleaving the peptide from the resin was performed in a BD syringe equipped with a frit. The cleavage cocktail consisting of TFA/TIS/EDT/ $\mathrm{H}_{2} \mathrm{O}(95: 2: 2: 1, v / v / v / v)$ was added to the resin and shaken at room temperature for at least $2.5 \mathrm{~h}$. Thereafter, the liquid was separated from the solid support using the frit in the BD syringe and the solid residue was rinsed with TFA to maximize peptide yield. The combined liquid phases were then concentrated in a nitrogen stream and afterwards precipitated with icecold diethyl ether. Using a Megafuge $8 R$ centrifuge from Thermo Fisher (Waltham, Massachusetts, USA), the peptide pellet was separated from the liquid phase. The precipitation process was performed three 
times. Finally, the crude peptide was dried for $30 \mathrm{~min}$ at ambient conditions and dissolved in minimal amounts of HFIP. The solution was diluted with ultrapure water (7-10 times the volume of HFIP), frozen in liquid nitrogen and freeze dried.

\subsection{Fusion Assays}

\subsubsection{Lipid Film Preparation}

For the lipid stock solutions either premade solutions were used or they were prepared by the solvation of solid lipids in chloroform. The stock solutions were stored at $-20{ }^{\circ} \mathrm{C}$. The concentration of 1,2-dioleoyl-sn-glycero-3-phosphocholine (18:1, $\Delta 9$-cis, DOPC), 1,2dioleoyl-sn-glycero-3-phosphoethanolamine (18:1, $\Delta$ 9-cis, DOPE) and cholesterol stock solution was $20 \mathrm{mg} / \mathrm{mL}$. The labeled lipids stock solutions of 1,2-dioleoyl-sn-glycero-3phosphoethanolamine- $N$-(7-nitro-2-1,3-benzoxadiazol-4-yl) (ammonium salt) (NBDDOPE), 1,2-dioleoyl-sn-glycero-3-phosphoethanolamine- $N$-(lissamine rhodamine B sulfonyl) (ammonium salt) (Rh-DOPE), 1,2-dihexadecanoyl-sn-glycero-3-phosphoethanolamine (Oregon green 488 DHPE) and 1,2-dihexadecanoyl-sn-glycero-3-phosphoethanolamine (triethylammonium salt) (Texas red DHPE) had a concentration of $1 \mathrm{mg} / \mathrm{mL}$. Peptides were dissolved in TFE and their concentration was determined prior to lipid film preparation via $\mathrm{UV} / \mathrm{Vis}$ absorption at $280 \mathrm{~nm}$ for uncaged peptides and $385 \mathrm{~nm}$ for caged peptides.

The lipid films were prepared in small glass tubes which were prefilled with chloroform. Test tubes containing labeled lipids were covered by aluminum foil to reduce the exposure to light. During the preparation the tubes and stock solutions were cooled in an ice bath. The stock solutions of lipids were added to the test tube with the appropriate volume to a total lipid amount of $625 \mathrm{nmol}$. The lipid composition varied depending on the experiment and are shown in Table 9.8. Subsequently, the peptide stock solutions were added according to the peptide to lipid ratio and TFE was added accordingly to reach $250 \mu \mathrm{L}$. Usually, $\mathrm{P} / \mathrm{L}=1: 500$ was used, leading to a total peptide amount of $1.25 \mathrm{nmol}$ per film. Lipid films without peptides were topped with pure TFE. After all components were added, the mixtures were mixed for $5 \mathrm{~s}$ and heated to $50{ }^{\circ} \mathrm{C}$. The solutions were vortexed again for $5 \mathrm{~s}$ and exposed to a slight nitrogen stream at $50{ }^{\circ} \mathrm{C}$. After the volatile solvent was evaporated, the lipid films were stored overnight in vacuo at $50{ }^{\circ} \mathrm{C}$ and used the next day. 
Table 9.8: List of the used lipid compositions in this thesis for the preparation of liposomes.

\begin{tabular}{ll} 
Liposome & Lipid composition (molar ratio) \\
\hline Unlabeled & DOPC/DOPE/Chol $=50: 25: 25$ \\
Unlabeled & DOPC/DOPE/DOPS/Chol = 50:20:20:10 \\
Unlabeled & DOPC/DOPE/PIP $/$ Chol = 50:25:0.1-2:25 \\
Labeled NBD+Rh & DOPC/DOPE/NBD-DOPE/Rh-DOPE/Chol = 50:22:1.5:1.5:25 \\
Labeled OG+TR & DOPC/DOPE/OG-DHPE/TR-DHPE/Chol = 50:22:1.5:1.5:25 \\
Labeled OG & DOPC/DOPE/OG-DHPE/Chol = 50:23.5:1.5:25 \\
Labeled TR & DOPC/DOPE/TR-DHPE/Chol = 50:23.5:1.5:25 \\
\hline
\end{tabular}

\subsubsection{Liposome Preparation via Extrusion}

Liposomes were prepared by the extrusion of the prepared films in HEPES buffer following standard procedure. ${ }^{[275]}$ The HEPES buffer (20 mM HEPES, $100 \mathrm{mM} \mathrm{KCl,} 1 \mathrm{~mm}$ EDTA, $1 \mathrm{mM}$ DTT, $\mathrm{pH}=7.4$ ) was prepared on the day of the experiment out of a stock solution consisting of a 10-fold stock of HEPES, $\mathrm{KCl}$ and EDTA in ultrapure water. After the addition of DTT, the $\mathrm{pH}$ was adjusted by the addition of $1 \mathrm{M} \mathrm{KOH}$. The HEPES buffer was filtered using a Chromafil $R C-45 / 15 M S$ syringe filter $(0.45 \mu \mathrm{m}$ pore size) from Macherey Nagel (Düren, Germany) and a fluorescence spectrum was measured to verify the quality. To the lipid films in the test tubes, $\sim 10$ glass beads ( $2 \mathrm{~mm}$ diameter) were added for better detachment of the glass wall. After the addition of $500 \mu \mathrm{L}$ buffer, the tubes were sealed with Parafilm and incubated at $40{ }^{\circ} \mathrm{C}$ while shaking at $190 \mathrm{rpm}$ for a minimum of $2.5 \mathrm{~h}$ on an Unimax 1010 platform shaker by Heidolph (Schwabach, Germany) equipped with the heating unit Incubator 1000.

For extrusion of the liposomes a LipoFast-Basic extruder from Avestin (Ottawa, Canada) was used with a $100 \mathrm{~nm}$ polycarbonate membrane either from Avestin or from GE Healthcare (Little Chalfont, UK). Additionally, Whatman polyester drain discs by GE Healthcare were used which were positioned before both membrane sides. The extruder was washed thoroughly before use with ethanol, ultrapure water and buffer. Next, the extruder was dried with paper tissues, assembled and filled with the buffer. All air bubbles were carefully removed during this process. After incubation, the lipid films were vortexed for $5 \mathrm{~s}$, treated in an ultrasonic bath for a maximum of $10 \mathrm{~s}$ and subsequently vortexed again for $5 \mathrm{~s}$. The homogeneous solution was filled inside one of the syringes of the extruder and 
extruded for 31 times. The liposome solution was added directly to a plastic tube or glass vial.

\subsubsection{FRET Dequenching Assay}

Detection of lipid mixing was performed on the FP-8200 fluorospectrometer by JASCO (Tokio, Japan) which was equipped with a magnetic stirrer and an injection port for direct injection of the sample. Temperature control was achieved by a ETC-272T peltier thermostat by JASCO connected to a Thermo Haake WKL26 water recirculator. Data was acquired using the provided software Spectra Manager (version 2.13.00). The sample was measured in a quartz glass cuvette with a path length of $10 \mathrm{~mm}$ for the excitation and $4 \mathrm{~mm}$ for the emission from Hellma Analytics (Müllheim, Germany).

The fusion experiments were performed at $25{ }^{\circ} \mathrm{C}$ with a stirring speed of $600 \mathrm{rpm}$. Two types of measurements were performed with the fluorophores NBD and Rh. First, fluorescence spectrum measurements were set to $460 \mathrm{~nm}$ excitation and emission was measured from $500-610 \mathrm{~nm}$. Second, time course measurements were performed with excitation at $460 \mathrm{~nm}$ and emission at $530 \mathrm{~nm}$ over $20 \mathrm{~min}$. In case of the use of Oregon green and Texas red as fluorophores, excitation was set to $495 \mathrm{~nm}$ and emission to $615 \mathrm{~nm}$ or $550-650 \mathrm{~nm}$ for spectrum measurements. Additional parameters were set according to Table 9.9.

Table 9.9: Parameters applied in fluorescence measurements. Values in parenthesis refer to measurements with Oregon green and Texas red.

\begin{tabular}{lll} 
Parameters & Spectrum & Time course \\
\hline Excitation & $460 \mathrm{~nm}(495 \mathrm{~nm})$ & $460 \mathrm{~nm}(495 \mathrm{~nm})$ \\
Ex bandwidth & $5 \mathrm{~nm}$ & $5 \mathrm{~nm}$ \\
Emission & $500-610 \mathrm{~nm}(550-650 \mathrm{~nm})$ & $530 \mathrm{~nm}(615 \mathrm{~nm})$ \\
Em bandwidth & $5 \mathrm{~nm}$ & $5 \mathrm{~nm}$ \\
Response & $0.5 \mathrm{~s}$ & $0.5 \mathrm{~s}$ \\
Sensitivity & high & high \\
Data interval & $1 \mathrm{~nm}$ & $1 \mathrm{~nm}$ \\
Scan speed/Measurement duration & $200 \mathrm{~nm} / \mathrm{min}$ & $1200 \mathrm{~s}$ \\
\hline
\end{tabular}

The measurements were performed according to the following standard operating procedure. First, a fluorescence spectrum of the buffer was recorded to obtain the value needed for background subtraction. For this, $1300 \mu \mathrm{L}$ were pipetted into the quartz cuvette. In case of deviations from the standard spectrum, the cuvette was washed again thoroughly with a solution of $3 \%$ Hellmanex III from Hellma (Mühlheim, Germany), ultrapure water 
and ethanol. Next, $10 \mu \mathrm{L}$ of the liposome sample with the fluorophores was added to the cuvette through the injection port with a Hamilton syringe and a spectrum was recorded to check whether the signal strength is enough for the measurement. After that, the time course measurement was started and $40 \mu \mathrm{L}$ of the unlabeled liposomes was added after exactly $30 \mathrm{~s}$ to the cuvette. In total, approximately $62.5 \mathrm{nmol}$ lipids were added the cuvette of which $12.5 \mathrm{nmol}$ are from the labeled sample and $50 \mathrm{nmol}$ of the unlabeled one. However, the actual values are lower, as lipid loss during extrusion is not accounted for. After 20 min, $1000 \mu \mathrm{L}$ of the sample is transferred to another cuvette for DLS measurements of the mixed population (see section 9.4.5) and retransferred to the quartz cuvette afterwards. A second time course measurement is started and around $\sim 15 \mathrm{~s}$ in, $30 \mu \mathrm{L}$ of a TritonX-100 solution $(2.5 \%$ in buffer $v / v)$ is added. After the signal stabilized, the measurement is aborted and a fluorescence spectrum from the mixed population is recorded. Here, the complete destruction of the vesicles is checked. The obtained data was subsequently analyzed according to equation 9.2.

$$
F=\frac{F_{\mathrm{t}}-F_{0}}{F_{\text {total }}-F_{0}}
$$

The fusion efficiency $F$ is calculated out of the recorded fluorescence $F_{\mathrm{t}}$ at the time $t$, the fluorescence before addition of the second liposome species $F_{0}$ and the total fluorescence after TritonX-100 addition $F_{\text {total }} . F_{0}$ is obtained by calculating the average of the last 10 data points before the addition. $F_{\text {total }}$ is the average of 50 data points after the signal stabilized. For visualization of the data, $F$ is plotted as the function of the time $t$.

\subsubsection{Uncaging experiments}

FRET dequenching assays with photocaged peptides were performed with the same instruments and generally with the same procedure as in section 9.4.3. Two types of uncaging experiments were performed during this thesis. In the following the differences to the standard FRET dequenching assay is described.

The first uncaging experiment starts with both liposome species being mixed in the fluorescence spectrometer. The photocaged peptide is not treated with a laser until this point. 5 min into the measurement, the fluorescence cuvette is taken out of the spectrometer and a handheld laser with a wavelength of $405 \mathrm{~nm}$ and $100 \mathrm{~mW}$ distributed by 
starklasers.com (London, UK) is pointed inside the top of the cuvette to illuminate the whole sample for $60 \mathrm{~s}$. Afterwards, the sample is put back into the spectrometer and the measurement is continued. The procedure is repeated after $500 \mathrm{~s}$ to verify if uncaging was complete.

In the second uncaging experiment, the sample with the liposome species containing the photocaged peptide was split into two parts. One part was set aside for DLS measurements of the sample without being exposed to the laser. The second part was exposed for $60 \mathrm{~s}$ to the handheld laser, right before injection into the fluorescence cuvette. Injection was done exactly $30 \mathrm{~s}$ into the time course measurement.

\subsubsection{Dynamic Light scattering}

DLS measurements were performed using a Zetasizer Nano $S$ by Malvern Panalytical (Kassel, Germany) with a $633 \mathrm{~nm}$ Laser at a probing angle of $173^{\circ}$. All data was acquired using the provided software. Each sample was measured at $25{ }^{\circ} \mathrm{C}$ using either disposable semi-micro or macro cuvettes from Brand GmbH (Wertheim, Germany). In case of semimicro cuvettes, single vesicle populations were measured using $100 \mu \mathrm{L}$ of the sample with $400 \mu \mathrm{L}$ of buffer. For mixed populations, $500 \mu \mathrm{L}$ of the sample was used. Using macro cuvettes, $900 \mu \mathrm{L}$ buffer was added for single vesicle populations. Mixed populations were measured using $1000 \mu \mathrm{L}$ of the sample. All measurements were performed as triplicates, while individual measurements were obtained with multiple runs between 12-18 times as determined by the software for optimal data quality. The collected data was processed by calculating the weighted arithmetic mean value of each measurement. The hydrodynamic radius $\left(d_{\mathrm{h}}\right)$ and PdI (Polydispersity index) were obtained using the instruments software.

\subsubsection{Phosphate Test}

The amount of lipids left in the sample after extrusion can be estimated with the phosphate test. Here, the total amount of phosphorus is determined of a small volume of the liposome sample and extrapolated for the whole sample. For this, a set of calibration samples with different specified quantity of dihydrogenphosphate, ranging from a total phosphorus amount of $0 \mu \mathrm{g}$ to $2 \mu \mathrm{g}$ were prepared from a stock solution $(\mathrm{c}=0.089 \mathrm{~g} / \mathrm{L})$. Out of these samples a calibration curve can be generated, which is used for the determination of the phosphorus content in the liposome samples. The samples were topped with ultrapure water 
to a total of $100 \mu \mathrm{L}$. For the liposome samples, $20 \mu \mathrm{L}$ and $200 \mu \mathrm{L}$ perchloric acid $(70 \%$ in water) were mixed in a glass vial. Every sample was made as duplicates and incubated at $220{ }^{\circ} \mathrm{C}$ for $60 \mathrm{~min}$ without cap. The vials were cooled to room temperature and $700 \mu \mathrm{L}$ of reagent $\mathrm{A}(0.45 \%$ ammonium orthomolybdate and $12.6 \%$ perchloric acid in water) and $700 \mu \mathrm{L}$ of reagent B (1.7\% ascorbic acid in water) were added. The vials were closed tightly with the caps and mixed thoroughly before incubating them in an $80{ }^{\circ} \mathrm{C}$ water bath for $7.5 \mathrm{~min}$. After the samples cooled to room temperature, they were transferred to single use cuvettes from Brand GmbH (Wertheim, Germany) and their absorbance at $820 \mathrm{~nm}$ was measured using a NanoDrop One C or NanoDrop 2000c. Using the calibration samples, a linear fit was applied and the calibration curve could be obtained. 


\subsection{Synthesized Compounds}

\subsubsection{Synthesized Molecules}

\section{7-Diethylamino-4-hydroxymethylcoumarin}

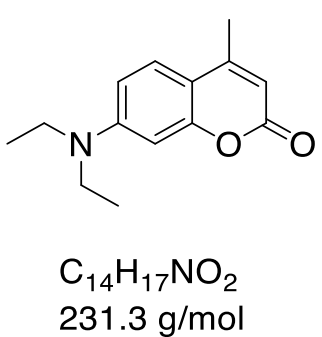

12 a) $\mathrm{SeO}_{2}$ xylene, $145^{\circ} \mathrm{C}, 48 \mathrm{~h}$

b) $\mathrm{NaBH}_{4}, \mathrm{MeOH}, \mathrm{RT}, 24 \mathrm{~h}$ $42 \%$

$$
\mathrm{C}_{14} \mathrm{H}_{17} \mathrm{NO}_{3}
$$

$247.3 \mathrm{~g} / \mathrm{mol}$

13

7-Diethylamino-4-methylcoumarin $(5.00 \mathrm{~g}, 21.6 \mathrm{mmol}, 1.00 \mathrm{eq}$.$) and selenium dioxide$ (2.72 g, $24.5 \mathrm{mmol}, 1.13$ eq.) were dissolved in xylene $(500 \mathrm{~mL})$ and stirred under reflux for $48 \mathrm{~h}$. The mixture was cooled to room temperature, filtered and the filtrate was reduced in vacuo. The residue was redissolved in methanol $(500 \mathrm{~mL})$ and $\mathrm{NaBH}_{4}(0.80 \mathrm{~g}$, $21.1 \mathrm{mmol}, 0.97$ eq.) was added to the mixture. After stirring at room temperature overnight, the unreacted $\mathrm{NaBH}_{4}$ was quenched by neutralizing with $\mathrm{HCl}(1 \mathrm{M})$. Methanol was removed at reduced pressure and the aqueous phase was extracted with DCM $(5 \mathrm{x}$ $100 \mathrm{~mL})$. The combined organic layers were washed with brine $(3 \times 80 \mathrm{~mL})$, dried over $\mathrm{NaSO}_{4}$ and the solvent was removed under reduced pressure. The crude product was purified by column chromatography (gradient of DCM/acetone $=19: 1 \rightarrow 5: 1$ ). The product (2.23 g, $9.00 \mathrm{mmol}, 42 \%)$ was obtained as a brown solid.

${ }^{1}$ H-NMR $\left(300 \mathrm{MHz}, \mathrm{CD}_{2} \mathrm{Cl}_{2}\right) ; \delta(\mathrm{ppm})=1.19\left(\mathrm{t}, J=7.1 \mathrm{~Hz}, 6 \mathrm{H}, \mathrm{CH}_{3}\right), 2.60$ (t, $J=6.0 \mathrm{~Hz}, 1 \mathrm{H}, \mathrm{OH}), 3.40\left(\mathrm{q}, J=7.1 \mathrm{~Hz}, 4 \mathrm{H}, \mathrm{CH}_{2}\right), 4.81\left(\mathrm{dd}, J=5.9,1.4 \mathrm{~Hz}, 2 \mathrm{H}, \mathrm{CH}_{2}-\right.$ $\mathrm{OH}), 6.18\left(\mathrm{~d}, J=1.4 \mathrm{~Hz}, 1 \mathrm{H}, \mathrm{CH}_{\text {arom }}\right), 6.47$ (d, $\left.J=2.6 \mathrm{~Hz}, 1 \mathrm{H}, \mathrm{CH}_{\text {arom }}\right), 6.58$ (dd, $J=9.0$, $\left.2.6 \mathrm{~Hz}, 1 \mathrm{H}, \mathrm{CH}_{\text {arom }}\right), 7.32$ (d, $\left.J=9.0 \mathrm{~Hz}, 1 \mathrm{H}, \mathrm{CH}_{\text {arom }}\right)$.

MS (ESI) $m / z: 248.1[\mathrm{M}+\mathrm{H}]^{+}, 270.1[\mathrm{M}+\mathrm{Na}]^{+}$.

HR-MS (ESI) $m / z$ : calc. for $\mathrm{C}_{14} \mathrm{H}_{18} \mathrm{NO}_{3}[\mathrm{M}+\mathrm{H}]^{+}: 248.1281$, found: 248.1282 ; calc. for $\mathrm{C}_{14} \mathrm{H}_{17} \mathrm{NO}_{3} \mathrm{Na}[\mathrm{M}+\mathrm{Na}]^{+}:$270.1101, found: 270.1104 . 


\section{(7-Diethylaminocoumarin-4-yl)methyl (4-nitrophenyl) carbonate}

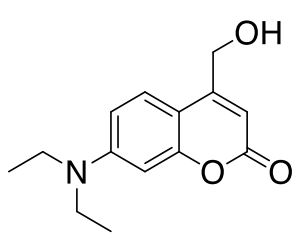

$\mathrm{C}_{14} \mathrm{H}_{17} \mathrm{NO}_{3}$

$247.3 \mathrm{~g} / \mathrm{mol}$

13
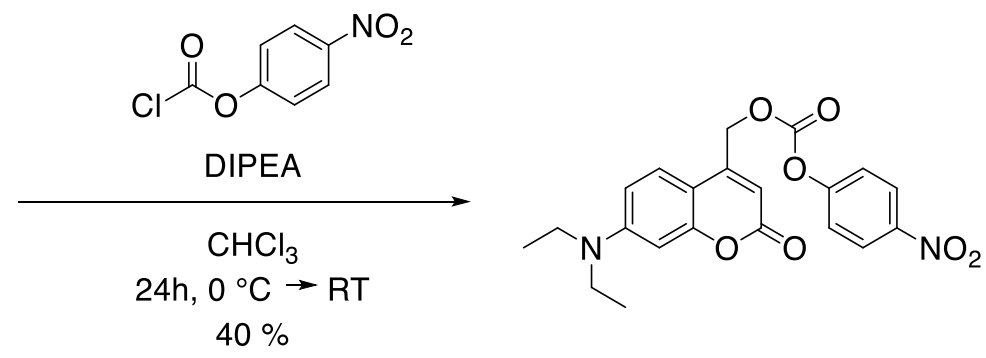

$\mathrm{C}_{21} \mathrm{H}_{20} \mathrm{~N}_{2} \mathrm{O}_{7}$ $412.4 \mathrm{~g} / \mathrm{mol}$

14

Under a nitrogen atmosphere, $13(1.80 \mathrm{~g}, 7.28 \mathrm{mmol}, 1.00$ eq.) and DIPEA (2.7 mL, $15.5 \mathrm{mmol}, 2.10$ eq.) were mixed in chloroform $(45 \mathrm{~mL})$. To the mixture 4-nitrophenyl chloroformate (3.08 g, $15.2 \mathrm{mmol}, 2.10$ eq.) and 4-dimethylaminopyridine (0.89 g, $7.28 \mathrm{mmol}$, 1.00 eq.) in THF $(80 \mathrm{~mL})$ were added. After the reaction mixture was stirred at room temperature for $24 \mathrm{~h}$, the solvent was removed in vacuo. The precipitate was dissolved in EtOAc $(300 \mathrm{~mL})$ and washed with $\mathrm{H}_{3} \mathrm{PO}_{4}(1 \mathrm{M}, 2 \times 80 \mathrm{~mL})$, water $(2 \times 80 \mathrm{~mL})$, saturated $\mathrm{NaHCO}_{3}$ solution $(2 \times 80 \mathrm{~mL})$ and brine $(2 \times 80 \mathrm{~mL})$ before being dried over $\mathrm{Na}_{2} \mathrm{SO}_{4}$. The organic phase was adsorbed on silica and the crude product was purified by column chromatography (gradient DCM $\rightarrow$ DCM/acetone =10:1). The product $(1.2 \mathrm{~g}, 2.91 \mathrm{mmol}$, $40 \%$ ) was obtained as a yellow solid.

${ }^{1} \mathbf{H}-\mathrm{NMR}(300 \mathrm{MHz}, \mathrm{DMSO}-\mathrm{d} 6) ; \delta(\mathrm{ppm})=1.13\left(\mathrm{t}, J=7.0 \mathrm{~Hz}, 6 \mathrm{H}, \mathrm{CH}_{3}\right), 3.45$ (q, $\left.J=7.0 \mathrm{~Hz}, 4 \mathrm{H}, \mathrm{CH}_{2}\right), 5.51\left(\mathrm{~d}, J=1.2 \mathrm{~Hz}, 2 \mathrm{H}, \mathrm{O}-\mathrm{CH}_{2}\right), 6.12\left(\mathrm{~s}, 1 \mathrm{H}, \mathrm{CH}_{\text {arom }}\right), 6.57$ (d, $\left.J=2.5 \mathrm{~Hz}, 1 \mathrm{H}, \mathrm{CH}_{\text {arom }}\right), 6.73\left(\mathrm{dd}, J=9.10 \mathrm{~Hz}, J=2.60 \mathrm{~Hz}, 1 \mathrm{H}, \mathrm{CH}_{\text {arom }}\right), 7.54(\mathrm{~d}, J=9.10$ $\mathrm{Hz}, 1 \mathrm{H}, \mathrm{CH}_{\text {arom }}$ ), 7.60-7.72 (m, $\left.2 \mathrm{H}, \mathrm{CH}_{\text {arom }}\right), 8.32-8.39$ (m, $2 \mathrm{H}, \mathrm{CH}_{\text {arom }}$ ).

MS (ESI) $m / z: 413.2[\mathrm{M}+\mathrm{H}]^{+}, 435.1[\mathrm{M}+\mathrm{Na}]^{+}$.

HR-MS (ESI) $m / z$ : calc. for $\mathrm{C}_{21} \mathrm{H}_{21} \mathrm{~N}_{2} \mathrm{O}_{7}[\mathrm{M}+\mathrm{H}]^{+}:$413.1346, found: 413.1345; calc. for $\mathrm{C}_{21} \mathrm{H}_{20} \mathrm{~N}_{2} \mathrm{O}_{7} \mathrm{Na}[\mathrm{M}+\mathrm{Na}]^{+}:$435.1163, found: 435.1166 . 
Fmoc-L-Lys(DEACM)-OH

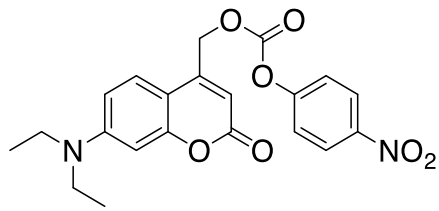

$\mathrm{C}_{21} \mathrm{H}_{20} \mathrm{~N}_{2} \mathrm{O}_{7}$

$412.4 \mathrm{~g} / \mathrm{mol}$

14

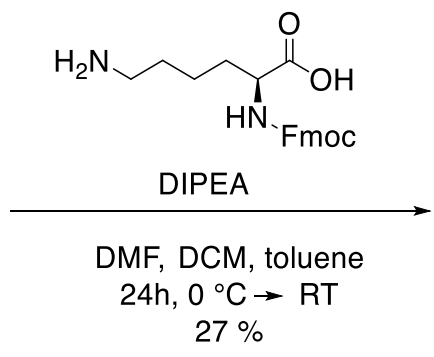

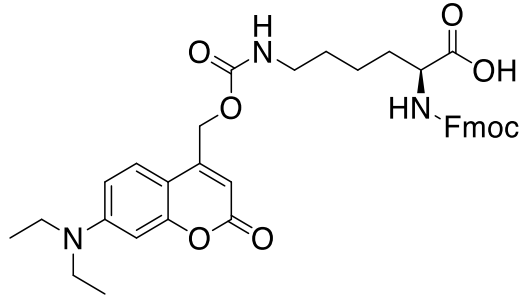

$\mathrm{C}_{36} \mathrm{H}_{39} \mathrm{~N}_{3} \mathrm{O}_{8}$

$641.7 \mathrm{~g} / \mathrm{mol}$

15

The reaction was performed under exclusion of light. To a suspension of $\mathbf{1 4}(1.2 \mathrm{~g}$, $2.91 \mathrm{mmol}, 1.0$ eq.) in DMF (30 mL) and DCM (30 mL), Fmoc-L-Lys-OH (1.07 g, $2.91 \mathrm{mmol}, 1.0$ eq. $)$ in toluene $(80 \mathrm{~mL})$ was added. The mixture was cooled to $0{ }^{\circ} \mathrm{C}$, DIPEA (510 $\mu \mathrm{L}, 2.93 \mathrm{mmol}, 1.0$ eq.) was added and it was stirred for $12 \mathrm{~h}$ while allowing it to slowly come to room temperature. Further Fmoc-L-Lys-OH (0.54 g, $1.46 \mathrm{mmol}, 0.5$ eq.) and DIPEA (250 $\mu \mathrm{L}, 1.44 \mathrm{mmol}, 0.5$ eq.) were added and stirred at room temperature overnight. After evaporation of the solvents in vacuo, the crude product was purified by column chromatography $(\mathrm{DCM} / \mathrm{MeOH} / \mathrm{AcOH}=97: 3: 0.1)$ to yield a light yellow solid $(0.50 \mathrm{~g}$, $0.78 \mathrm{mmol}, 27 \%)$.

${ }^{1}$ H-NMR $(300 \mathrm{MHz}, \mathrm{DMSO}-\mathrm{d} 6) ; \delta(\mathrm{ppm})=1.11\left(\mathrm{t}, J=6.9 \mathrm{~Hz}, 6 \mathrm{H}, \mathrm{CH}_{3}\right), 1.22-1.81(\mathrm{~m}$, $\left.6 \mathrm{H}, \mathrm{CH}_{2}\right), 3.04\left(\mathrm{q}, J=6.4 \mathrm{~Hz}, 2 \mathrm{H}, \mathrm{CH}_{2}\right), 3.43\left(\mathrm{t}, J=7.0 \mathrm{~Hz}, 4 \mathrm{H}, \mathrm{CH}_{2}-\mathrm{CH}_{3}\right), 3.86-4.00$ (m, $1 \mathrm{H}, \mathrm{CH}), 4.18-4.33$ (m, $3 \mathrm{H}$, Fmoc-CH, Fmoc-CH $), 5.20$ (s, $\left.2 \mathrm{H}, \mathrm{CH}_{2}\right), 5.97$ (s, $1 \mathrm{H}$, $\left.\mathrm{CH}_{\text {Arom }}\right), 6.53\left(\mathrm{~d}, J=2.5 \mathrm{~Hz}, 1 \mathrm{H}, \mathrm{CH}_{\text {Arom }}\right), 6.67$ (dd, $\left.J=9.1,2.5 \mathrm{~Hz}, 1 \mathrm{H}, \mathrm{CH}_{\text {Arom }}\right), 7.33$ (td, $J=7.4,1.2 \mathrm{~Hz}, 2 \mathrm{H}$, Fmoc- $\left.\mathrm{CH}_{\text {Arom }}\right), 7.38-7.46$ (m, $3 \mathrm{H}, \mathrm{Fmoc}-\mathrm{CH}_{\text {Arom }}, \mathrm{CH}_{\text {Arom }}$ ), 7.52 $\left(\mathrm{t}, J=5.7 \mathrm{~Hz}, 1 \mathrm{H}, \mathrm{NH}-\mathrm{CH}_{2}\right), 7.62(\mathrm{~d}, J=8.0 \mathrm{~Hz}, 1 \mathrm{H}, \mathrm{NH}-\mathrm{CH}), 7.73(\mathrm{~d}, J=7.4 \mathrm{~Hz}, 2 \mathrm{H}$, Fmoc- $\left.\mathrm{CH}_{\text {Arom }}\right), 7.89$ (d, $J=7.4 \mathrm{~Hz}, 2 \mathrm{H}$, Fmoc- $\left.\mathrm{CH}_{\text {Arom }}\right)$.

MS (ESI) $m / z: 664.3[\mathrm{M}+\mathrm{Na}]^{+}, 1305.5[2 \mathrm{M}+\mathrm{Na}]^{+}$.

HR-MS (ESI) $m / z$ : calc. for $\mathrm{C}_{36} \mathrm{H}_{40} \mathrm{~N}_{3} \mathrm{O}_{8}[\mathrm{M}+\mathrm{H}]^{+}:$642.2810, found: 642.2791; calc. for $\mathrm{C}_{36} \mathrm{H}_{39} \mathrm{~N}_{3} \mathrm{O}_{8} \mathrm{Na}[\mathrm{M}+\mathrm{Na}]^{+}:$664.2629, found: 664.2607 . 


\title{
9.5.2 Synthesized Peptides
}

\section{E3-Syb(RKY)}

\author{
H-G-E I AALEK-E I AALEK-E I AALEK-RKYWWKNLK- \\ MM I I LGV I CA I I L I I I I VYFST-OH \\ $\mathrm{C}_{288} \mathrm{H}_{473} \mathrm{~N}_{65} \mathrm{O}_{71} \mathrm{~S}_{3}$ \\ $6078.5 \mathrm{~g} / \mathrm{mol}$ \\ 1
}

The peptide was synthesized according to section 9.3.1 at a scale of $50 \mu \mathrm{mol}$. After cleavage and lyophilization the peptide was purified with HPLC Semi-Prep using column 2 and a twostep gradient of solvent system I (75-85 \% B in 3 min, 85-99 \% B in 15 min).

UPLC: (Column 1, solvent system II; 70-99 \% B in 8 min) $R_{t}=9.6$ min.

MS (ESI) $m / z: 1014.0[\mathrm{M}+6 \mathrm{H}]^{6+}, 1216.5[\mathrm{M}+5 \mathrm{H}]^{5+}, 1520.3[\mathrm{M}+4 \mathrm{H}]^{4+}$.

HR-MS (ESI) $m / z$ : calc. for $\mathrm{C}_{288} \mathrm{H}_{479} \mathrm{~N}_{65} \mathrm{O}_{71} \mathrm{~S}_{3}\left([\mathrm{M}+6 \mathrm{H}]^{6+}\right)$ : 1014.0851, found: 1014.0848; calc. for $\mathrm{C}_{288} \mathrm{H}_{478} \mathrm{~N}_{65} \mathrm{O}_{71} \mathrm{~S}_{3}\left([\mathrm{M}+5 \mathrm{H}]^{5+}\right)$ : 1216.7006, found: 1216.7011; calc. for $\mathrm{C}_{288} \mathrm{H}_{477} \mathrm{~N}_{65} \mathrm{O}_{71} \mathrm{~S}_{3}\left([\mathrm{M}+4 \mathrm{H}]^{4+}\right): 1520.3734$, found: 1520.3714 .

\section{E3-Syb(RKYAW)}

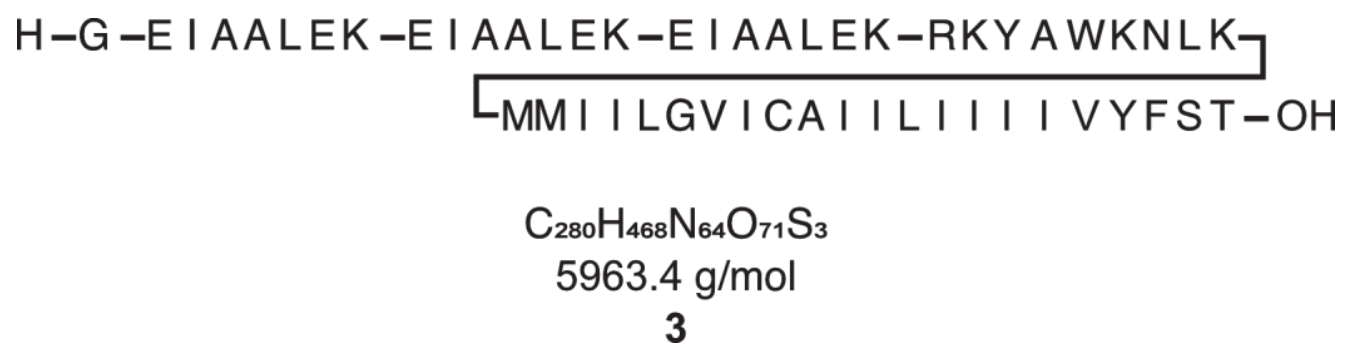

The peptide was synthesized according to section 9.3.1 at a scale of $50 \mu \mathrm{mol}$. After cleavage and lyophilization the peptide was purified with HPLC Prep using column 3 and a twostep gradient of solvent system I (75-85 \% B in 3 min, 85-99 \% B in 15 min).

UPLC: (Column 1, solvent system II; 70-99 \% B in 8 min) $R_{t}=8.8$ min. 
MS (ESI) $m / z: 994.7[\mathrm{M}+6 \mathrm{H}]^{6+}, 1193.5[\mathrm{M}+5 \mathrm{H}]^{5+}$.

HR-MS (ESI) $m / z$ : calc. for $\mathrm{C}_{280} \mathrm{H}_{474} \mathrm{~N}_{64} \mathrm{O}_{71} \mathrm{~S}_{3}\left([\mathrm{M}+6 \mathrm{H}]^{6+}\right)$ : 994.7443, found: 994.7480; calc. for $\mathrm{C}_{280} \mathrm{H}_{474} \mathrm{~N}_{64} \mathrm{O}_{71} \mathrm{~S}_{3}\left([\mathrm{M}+5 \mathrm{H}]^{5+}\right)$ : 1193.4917 , found: 1193.4943 .

\section{E3-Syb(RKYWA)}

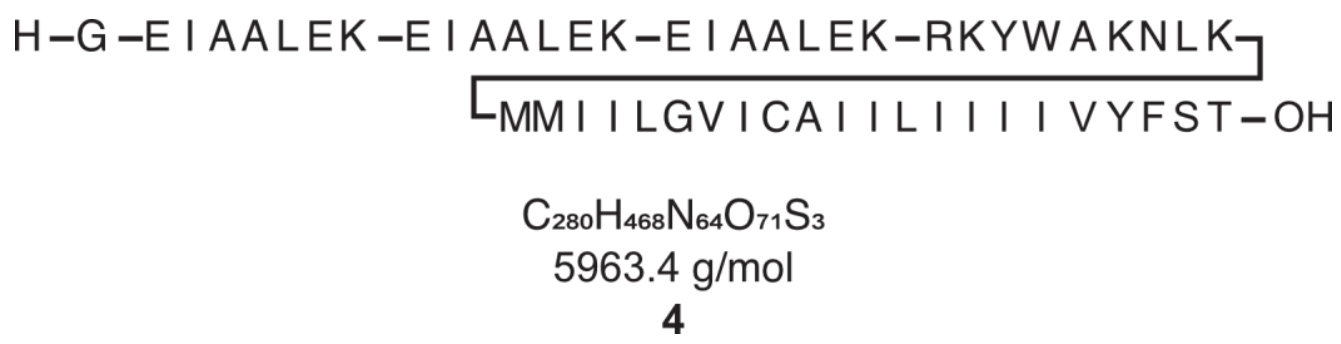

The peptide was synthesized according to section 9.3.1 at a scale of $50 \mu \mathrm{mol}$. After cleavage and lyophilization the peptide was purified with HPLC Prep using column 3 and a twostep gradient of solvent system I (75-85 \% B in 3 min, 85-99 \% B in 15 min).

UPLC: (Column 1, solvent system II; 70-99 \% B in 8 min) $\mathrm{R}_{\mathrm{t}}=8.6 \mathrm{~min}$.

MS (ESI) $m / z: 1193.6[\mathrm{M}+5 \mathrm{H}]^{5+}, 1491.8[\mathrm{M}+4 \mathrm{H}]^{4+}$.

HR-MS (ESI) $m / z$ : calc. for $\mathrm{C}_{280} \mathrm{H}_{473} \mathrm{~N}_{64} \mathrm{O}_{71} \mathrm{~S}_{3}\left([\mathrm{M}+5 \mathrm{H}]^{5+}\right)$ : 1193.6922, found: 1193.6931; calc. for $\mathrm{C}_{280} \mathrm{H}_{472} \mathrm{~N}_{64} \mathrm{O}_{71} \mathrm{~S}_{3}\left([\mathrm{M}+4 \mathrm{H}]^{4+}\right)$ : 1491.8634 , found: 1491.8639 .

\section{E3-Syb(RKYAA)}

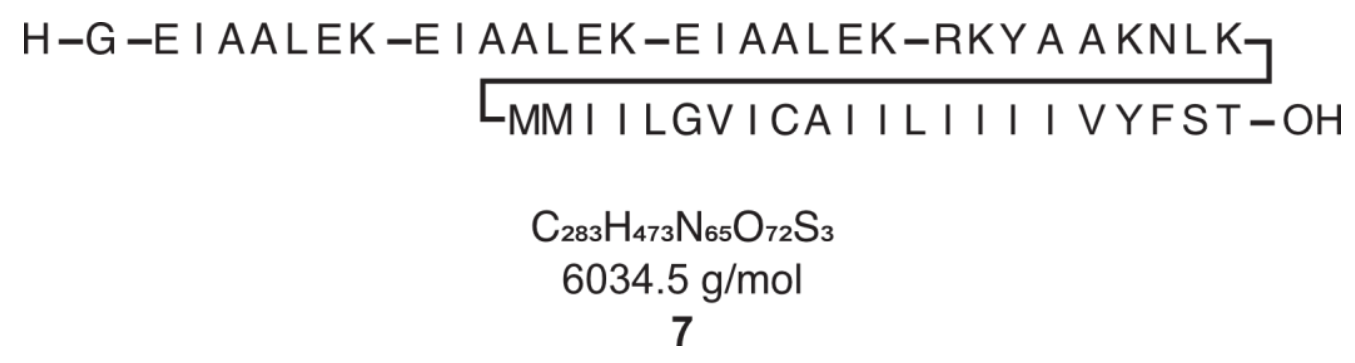

The peptide was synthesized according to section 9.3.1 at a scale of $50 \mu \mathrm{mol}$. After cleavage and lyophilization the peptide was purified with HPLC Prep using column 3 and a twostep gradient of solvent system I (75-85 \% B in 3 min, 85-99 \% B in 15 min). 
UPLC: (Column 1, solvent system II; 70-99 \% B in 8 min) $\mathrm{R}_{\mathrm{t}}=8.5 \mathrm{~min}$.

MS (ESI) $m / z: 862.9[\mathrm{M}+7 \mathrm{H}]^{7+}, 1006.7[\mathrm{M}+6 \mathrm{H}]^{6+}, 1207.7[\mathrm{M}+5 \mathrm{H}]^{5+}, 1509.3[\mathrm{M}+4 \mathrm{H}]^{4+}$.

HR-MS (ESI) $m / z$ : calc. for $\mathrm{C}_{283} \mathrm{H}_{480} \mathrm{~N}_{65} \mathrm{O}_{72} \mathrm{~S}_{3}\left([\mathrm{M}+7 \mathrm{H}]^{7+}\right)$ : 862.9300, found: 862.9289; calc. for $\mathrm{C}_{283} \mathrm{H}_{479} \mathrm{~N}_{65} \mathrm{O}_{72} \mathrm{~S}_{3}\left([\mathrm{M}+6 \mathrm{H}]^{6+}\right)$ : 1006.7509, found: 1006.7500 ; calc. for $\mathrm{C}_{283} \mathrm{H}_{478} \mathrm{~N}_{65} \mathrm{O}_{72} \mathrm{~S}_{3}\left([\mathrm{M}+5 \mathrm{H}]^{5+}\right): 1207.6991$, found: 1207.6981 ; calc. for $\mathrm{C}_{283} \mathrm{H}_{477} \mathrm{~N}_{65} \mathrm{O}_{72} \mathrm{~S}_{3}$ $\left([\mathrm{M}+4 \mathrm{H}]^{4+}\right): 1509.3721$, found: 1509.3711 .

\section{E3-Syb(RKA)}

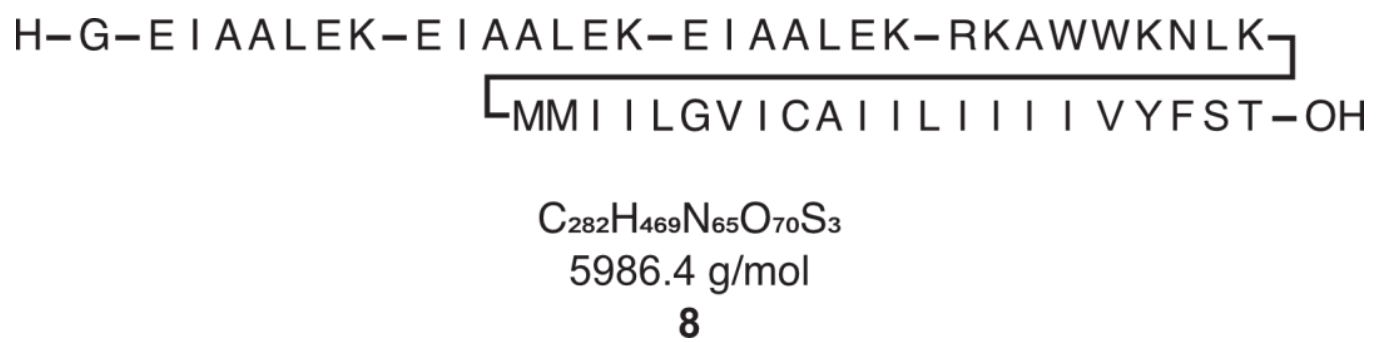

The peptide was synthesized according to section 9.3.1 at a scale of $50 \mu \mathrm{mol}$. After cleavage and lyophilization the peptide was purified with HPLC Semi-Prep using column 2 and a gradient of solvent system I (75-85 \% B in 3 min, 85-99 \% B in 15 min).

UPLC: (Column 1, solvent system II; 70-99 \% B in 8 min) $\mathrm{R}_{\mathrm{t}}=9.1 \mathrm{~min}$.

MS (ESI) $m / z: 998.6[\mathrm{M}+6 \mathrm{H}]^{6+}, 1198.3[\mathrm{M}+5 \mathrm{H}]^{5+}, 1497.6[\mathrm{M}+4 \mathrm{H}]^{4+}$.

HR-MS (ESI) $m / z$ : calc. for $\mathrm{C}_{282} \mathrm{H}_{475} \mathrm{~N}_{65} \mathrm{O}_{70} \mathrm{~S}_{3}\left([\mathrm{M}+6 \mathrm{H}]^{6+}\right)$ : 998.5803, found: 998.5802; calc. for $\mathrm{C}_{282} \mathrm{H}_{474} \mathrm{~N}_{65} \mathrm{O}_{70} \mathrm{~S}_{3}\left([\mathrm{M}+5 \mathrm{H}]^{5+}\right)$ : 1198.4958 , found: 1198.4924 ; calc. for $\mathrm{C}_{282} \mathrm{H}_{473} \mathrm{~N}_{65} \mathrm{O}_{70} \mathrm{~S}_{3}\left([\mathrm{M}+4 \mathrm{H}]^{4+}\right)$ : 1497.6174 , found: 1497.6186 . 


\section{K3-Sx(QSK)}

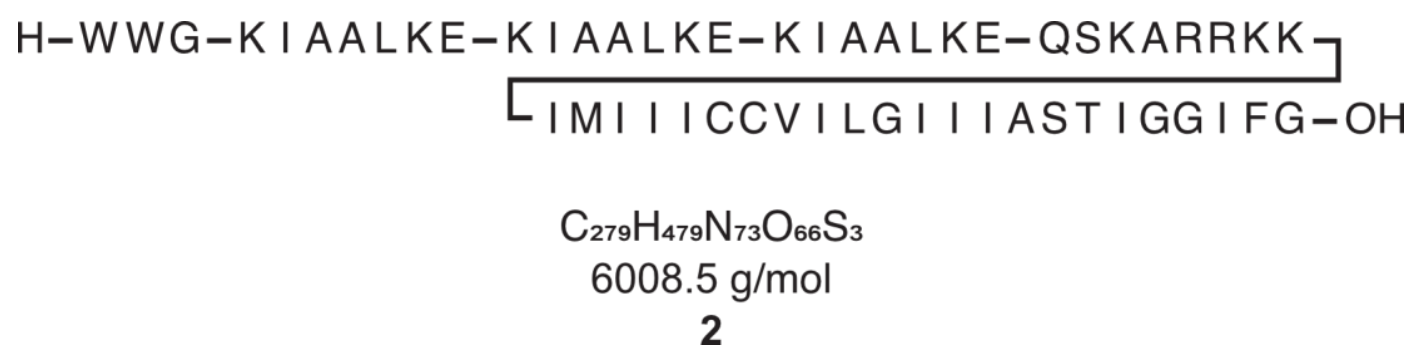

The peptide was synthesized according to section 9.3.1 at a scale of $50 \mu \mathrm{mol}$. After cleavage and lyophilization the peptide was purified with HPLC Semi-Prep using column 2 and a twostep gradient of solvent system I (75-85 \% B in 3 min, 85-99 \% B in 15 min).

UPLC: (Column 1, solvent system II; 70-99 \% B in 8 min) $\mathrm{R}_{\mathrm{t}}=6.4 \mathrm{~min}$.

MS (ESI) $m / z: 668.5[\mathrm{M}+9 \mathrm{H}]^{9+}, 752.1[\mathrm{M}+8 \mathrm{H}]^{8+}, 859.2[\mathrm{M}+7 \mathrm{H}]^{7+}, 1002.3[\mathrm{M}+6 \mathrm{H}]^{6+}$.

HR-MS (ESI) $m / z$ : calc. for $\mathrm{C}_{279} \mathrm{H}_{488} \mathrm{~N}_{73} \mathrm{O}_{66} \mathrm{~S}_{3}\left([\mathrm{M}+9 \mathrm{H}]^{9+}\right)$ : 668.5141, found: 668.5128; calc. for $\mathrm{C}_{279} \mathrm{H}_{487} \mathrm{~N}_{73} \mathrm{O}_{66} \mathrm{~S}_{3}\left([\mathrm{M}+8 \mathrm{H}]^{8+}\right): \quad 7$ 52.0777, found: 752.0765; calc. for $\mathrm{C}_{279} \mathrm{H}_{486} \mathrm{~N}_{73} \mathrm{O}_{66} \mathrm{~S}_{3}\left([\mathrm{M}+7 \mathrm{H}]^{7+}\right): 859.2303$, found: 859.2290; calc. for $\mathrm{C}_{279} \mathrm{H}_{485} \mathrm{~N}_{73} \mathrm{O}_{66} \mathrm{~S}_{3}$ $\left([\mathrm{M}+6 \mathrm{H}]^{6+}\right): 1002.2675$, found: 1002.2662 .

\section{K3-SX(QSXARRXK)}

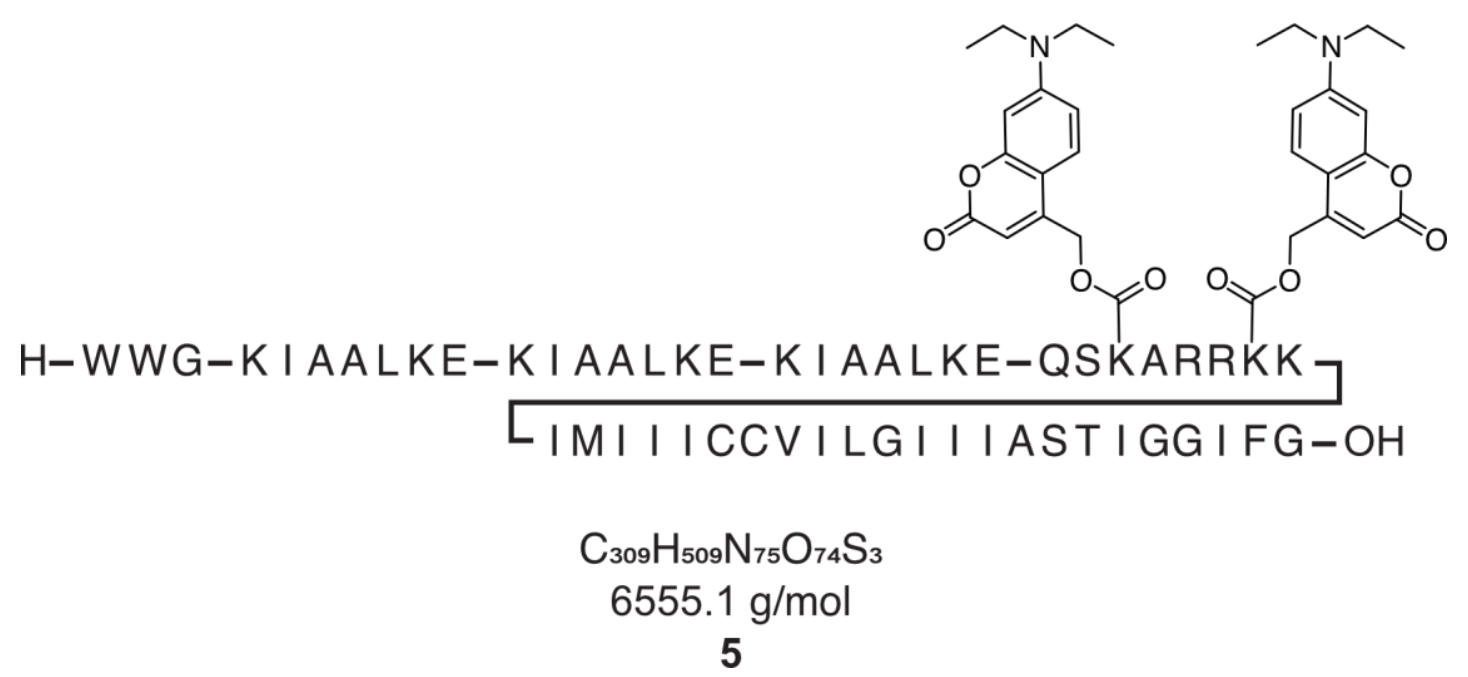

The peptide was synthesized according to section 9.3.1 at a scale of $10 \mu \mathrm{mol}$. To the activator base additionally DIPEA ( $0.02 \mathrm{M})$ was added. Coupling of each caged amino acid was performed twice. The peptide and caged amino acids were handled under exclusion of 
light. After cleavage and lyophilization the peptide was purified with HPLC Semi-Prep using column 2 and a twostep gradient of solvent system I (75-85 \% B in 3 min, 85-99 \% $\mathrm{B}$ in $15 \mathrm{~min})$.

UPLC: (Column 1, solvent system II; 25-65\% B in $3 \mathrm{~min}, 65-95 \% \mathrm{~B}$ in $12 \mathrm{~min}$ ) $\mathrm{R}_{\mathrm{t}}=15.0 \mathrm{~min}$.

MS (ESI) $m / z: 1093.3[\mathrm{M}+6 \mathrm{H}]^{6+}, 1097.1[\mathrm{M}+5 \mathrm{H}+\mathrm{Na}]^{6+}, 1311.9[\mathrm{M}+5 \mathrm{H}]^{5+}$.

HR-MS (ESI) $m / z$ : calc. for $\mathrm{C}_{309} \mathrm{H}_{515} \mathrm{~N}_{75} \mathrm{O}_{74} \mathrm{~S}_{3}\left([\mathrm{M}+6 \mathrm{H}]^{6+}\right)$ : 1093.3009 , found: 1093.2952; calc. for $\mathrm{C}_{309} \mathrm{H}_{514} \mathrm{~N}_{75} \mathrm{O}_{74} \mathrm{~S}_{3} \mathrm{Na}\left([\mathrm{M}+5 \mathrm{H}+\mathrm{Na}]^{6+}\right)$ : 1097.1316 , found: 1097.1288 ; calc. for $\mathrm{C}_{309} \mathrm{H}_{514} \mathrm{~N}_{75} \mathrm{O}_{74} \mathrm{~S}_{3}\left([\mathrm{M}+5 \mathrm{H}]^{5+}\right)$ : 1311.9601 , found: 1311.9572 .

\section{K3-Sx(KYQSK)}

\section{H-WWG-K I AALKE-K I AALKE-K I AALKE-KYQSKARRKK - IM I I I CCV ILGI I I ASTIGG I FG-OH \\ $\mathrm{C}_{294} \mathrm{H}_{500} \mathrm{~N}_{76} \mathrm{O}_{69} \mathrm{~S}_{3}$ $6299.9 \mathrm{~g} / \mathrm{mol}$ \\ 6}

The peptide was synthesized according to section 9.3.1 at a scale of $50 \mu \mathrm{mol}$. After cleavage and lyophilization the peptide was purified with HPLC Semi-Prep using column 2 and a twostep gradient of solvent system I (75-85 \% B in 3 min, 85-99 \% B in 15 min).

UPLC: (Column 1, solvent system II; 70-99 \% B in 8 min) $R_{t}=6.1$ min.

MS (ESI) $m / z: 788.41[\mathrm{M}+8 \mathrm{H}]^{8+}, 901.0[\mathrm{M}+7 \mathrm{H}]^{7+}, 1051.0[\mathrm{M}+6 \mathrm{H}]^{6+} 1260.8[\mathrm{M}+5 \mathrm{H}]^{5+}$.

HR-MS (ESI) $m / z$ : calc. for $\mathrm{C}_{294} \mathrm{H}_{508} \mathrm{~N}_{76} \mathrm{O}_{69} \mathrm{~S}_{3}\left([\mathrm{M}+8 \mathrm{H}]^{8+}\right)$ : 788.4725, found: 788.4731; calc. for $\mathrm{C}_{294} \mathrm{H}_{507} \mathrm{~N}_{76} \mathrm{O}_{69} \mathrm{~S}_{3}\left([\mathrm{M}+7 \mathrm{H}]^{7+}\right)$ : 900.9675, found: 900.9685; calc. for $\mathrm{C}_{294} \mathrm{H}_{506} \mathrm{~N}_{76} \mathrm{O}_{69} \mathrm{~S}_{3}\left([\mathrm{M}+6 \mathrm{H}]^{6+}\right): 1050.7939$, found: 1050.7954 ; calc. for $\mathrm{C}_{294} \mathrm{H}_{505} \mathrm{~N}_{76} \mathrm{O}_{69} \mathrm{~S}_{3}$ $\left([\mathrm{M}+5 \mathrm{H}]^{5+}\right): 1260.9516$, found: 1260.9520 . 


\section{K3-Sx(KAQSK)}

H-WWG-K I AALKE-K I AALKE-K I AALKE-KAQSKARRKK

IM I I I CCV I LG I I I AST IGG I FG-OH

\section{$\mathrm{C}_{288} \mathrm{H}_{496} \mathrm{~N}_{76} \mathrm{O}_{68} \mathrm{~S}_{3}$}

$6203.7 \mathrm{~g} / \mathrm{mol}$

\section{9}

The peptide was synthesized according to section 9.3.1 at a scale of $50 \mu \mathrm{mol}$. After cleavage and lyophilization the peptide was purified with HPLC Semi-Prep using column 2 and a twostep gradient of solvent system I (75-85 \% B in 3 min, 85-99 \% B in $15 \mathrm{~min}$ ).

UPLC: (Column 1, solvent system II; 70-99 \% B in 8 min) $\mathrm{R}_{\mathrm{t}}=6.6 \mathrm{~min}$.

MS (ESI) $m / z: 776.8[\mathrm{M}+8 \mathrm{H}]^{8+}, 887.7[\mathrm{M}+7 \mathrm{H}]^{7+}, 1051.0[\mathrm{M}+6 \mathrm{H}]^{6+}$.

HR-MS (ESI) $m / z$ : calc. for $\mathrm{C}_{288} \mathrm{H}_{504} \mathrm{~N}_{76} \mathrm{O}_{68} \mathrm{~S}_{3}\left([\mathrm{M}+8 \mathrm{H}]^{8+}\right)$ : 776.8439, found: 776.8422; calc. for $\mathrm{C}_{288} \mathrm{H}_{503} \mathrm{~N}_{76} \mathrm{O}_{68} \mathrm{~S}_{3}\left([\mathrm{M}+7 \mathrm{H}]^{7+}\right):$ 887.6777, found: 887.6794; calc. for $\mathrm{C}_{288} \mathrm{H}_{502} \mathrm{~N}_{76} \mathrm{O}_{68} \mathrm{~S}_{3}\left([\mathrm{M}+6 \mathrm{H}]^{6+}\right)$ : 1035.6232 , found: 1035.6197.

\section{K3-Sx(QSKAAAK)}

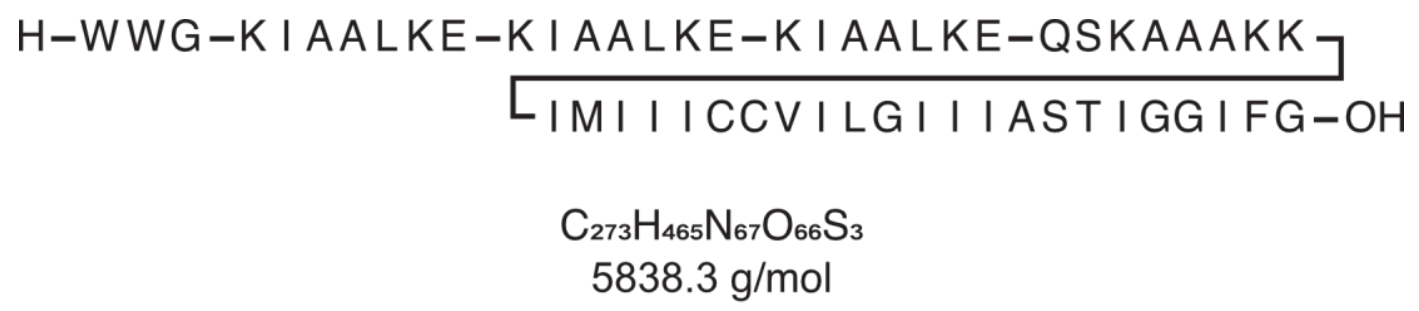

10

The peptide was synthesized according to section 9.3.1 at a scale of $50 \mu \mathrm{mol}$. After cleavage and lyophilization the peptide was purified with HPLC Prep using column 3 and a twostep gradient of solvent system I (75-85 \% B in $3 \mathrm{~min}, 85-99 \%$ B in $15 \mathrm{~min})$.

UPLC: (Column 1, solvent system II; 70-99 \% B in 8 min) $\mathrm{R}_{\mathrm{t}}=7.5 \mathrm{~min}$.

MS (ESI) $m / z: 834.9[\mathrm{M}+7 \mathrm{H}]^{7+}, 973.9[\mathrm{M}+6 \mathrm{H}]^{6+}, 1168.5[\mathrm{M}+5 \mathrm{H}]^{5+}, 1460.6[\mathrm{M}+4 \mathrm{H}]^{4+}$.

HR-MS (ESI) $m / z$ : calc. for $\mathrm{C}_{273} \mathrm{H}_{472} \mathrm{~N}_{67} \mathrm{O}_{66} \mathrm{~S}_{3}\left([\mathrm{M}+7 \mathrm{H}]^{7+}\right)$ : 834.9263, found: 834.9268; calc. for $\mathrm{C}_{273} \mathrm{H}_{471} \mathrm{~N}_{67} \mathrm{O}_{66} \mathrm{~S}_{3}\left([\mathrm{M}+6 \mathrm{H}]^{6+}\right)$ : 973.9128, found: 973.9135; calc. for 
$\mathrm{C}_{273} \mathrm{H}_{470} \mathrm{~N}_{67} \mathrm{O}_{66} \mathrm{~S}_{3}\left([\mathrm{M}+5 \mathrm{H}]^{5+}\right): 1168.4939$, found: 1168.4952 ; calc. for $\mathrm{C}_{273} \mathrm{H}_{469} \mathrm{~N}_{67} \mathrm{O}_{66} \mathrm{~S}_{3}$ $\left([\mathrm{M}+4 \mathrm{H}]^{4+}\right):$ 1460.6161, found: 1460.6186 .

\section{K3-Sx(QSAARRA)}

$$
\begin{aligned}
& \text { H-WWG-K I A A LKE-K I A A LKE-K I A A LKE-QSAARRAK } \\
& \qquad \begin{array}{c}
\hline \text { M I I I CCV I LG I I I AST I GG I FG-OH } \\
\mathrm{C}_{273} \mathrm{H}_{465} \mathrm{~N}_{71} \mathrm{O}_{66} \mathrm{~S}_{3} \\
5894.3 \mathrm{~g} / \mathrm{mol} \\
11
\end{array}
\end{aligned}
$$

The peptide was synthesized according to section 9.3.1 at a scale of $50 \mu \mathrm{mol}$. After cleavage and lyophilization the peptide was purified with HPLC Prep using column 3 and a twostep gradient of solvent system I (75-85 \% B in $3 \mathrm{~min}, 85-99 \%$ B in $15 \mathrm{~min}$ ).

UPLC: (Column 1, solvent system II; 70-99 \% B in 8 min) $\mathrm{R}_{t}=7.5 \mathrm{~min}$.

MS (ESI) $m / z: 842.9[\mathrm{M}+7 \mathrm{H}]^{7+}, 983.2[\mathrm{M}+6 \mathrm{H}]^{6+}, 1474.3[\mathrm{M}+4 \mathrm{H}]^{4+}$.

HR-MS (ESI) $m / z$ : calc. for $\mathrm{C}_{273} \mathrm{H}_{472} \mathrm{~N}_{71} \mathrm{O}_{66} \mathrm{~S}_{3}\left([\mathrm{M}+7 \mathrm{H}]^{7+}\right)$ : 842.9280, found: 842.9296; calc. for $\mathrm{C}_{273} \mathrm{H}_{471} \mathrm{~N}_{71} \mathrm{O}_{66} \mathrm{~S}_{3} \quad\left([\mathrm{M}+6 \mathrm{H}]^{6+}\right)$ : 983.2482, found: 983.2496; calc. for $\mathrm{C}_{273} \mathrm{H}_{469} \mathrm{~N}_{71} \mathrm{O}_{66} \mathrm{~S}_{3}\left([\mathrm{M}+4 \mathrm{H}]^{4+}\right)$ : 1474.3686 , found: 1474.372 . 



\section{Appendix}

\subsection{FRET Dequenching Assay Data}

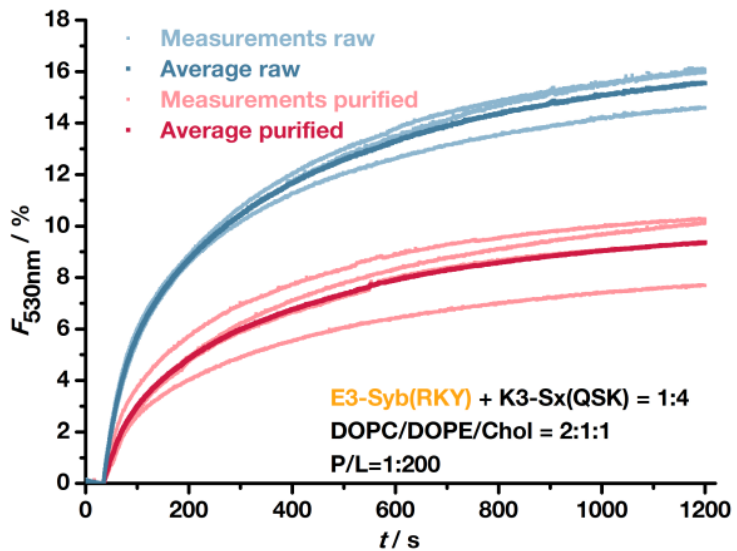

Additional data for Figure 4.3.

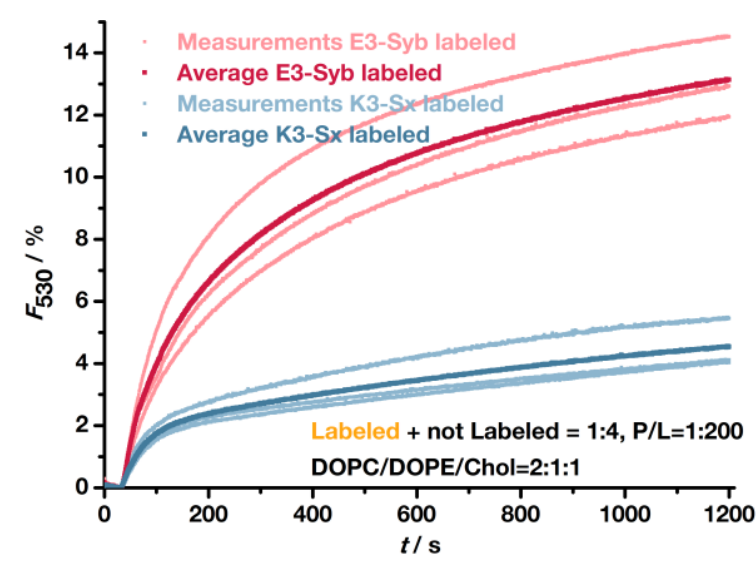

Additional data for Figure 4.7.

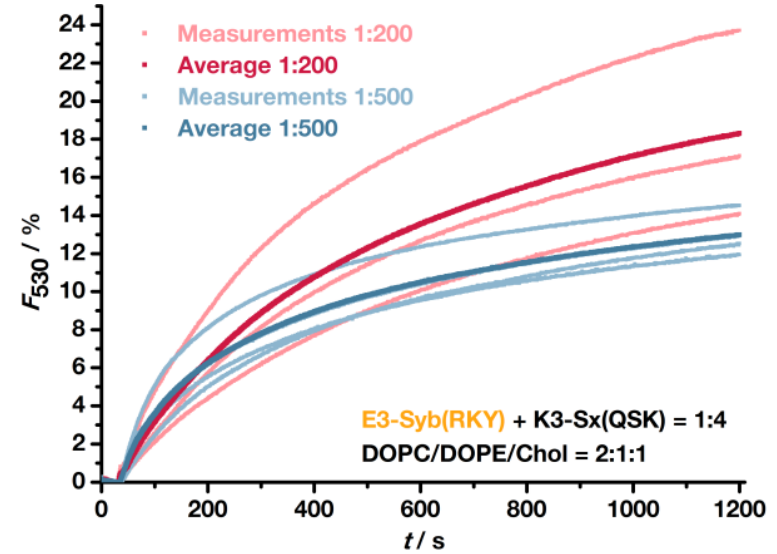

Additional data for Figure 4.5.

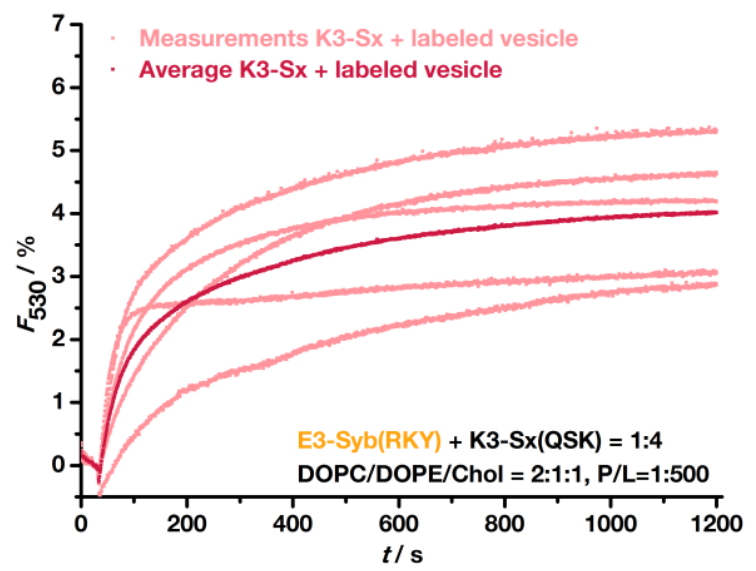

Additional data for Figure 4.8.

Figure A1: Individual fusion curves from FRET dequenching assays. The corresponding figures are indicated for each graph. 


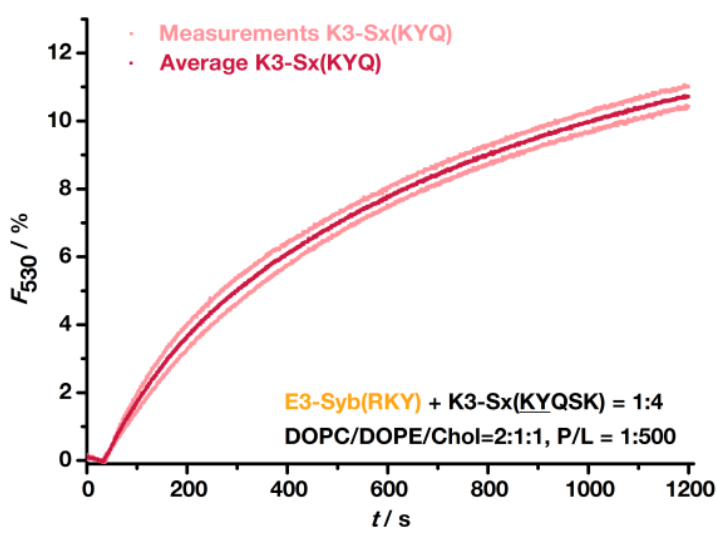

Additional data for Figure 5.2.

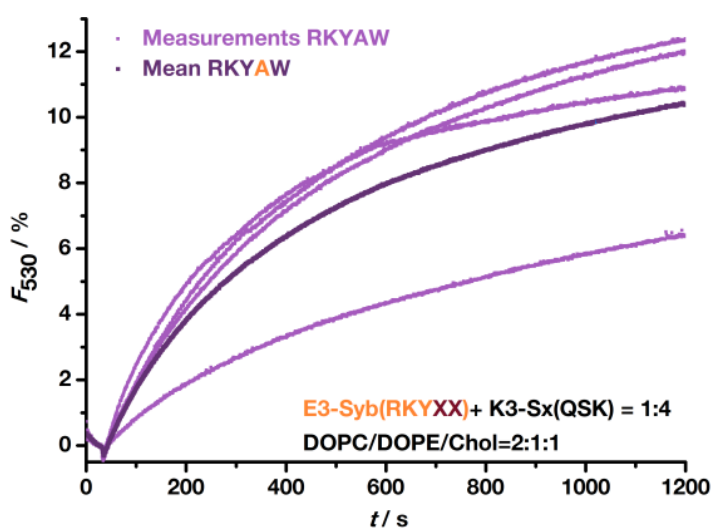

Additional data for Figure 5.4.

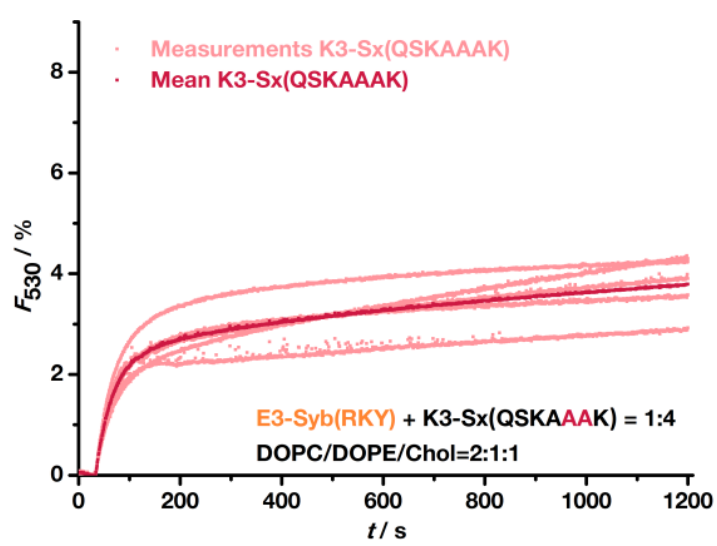

Additional data for Figure 5.9.

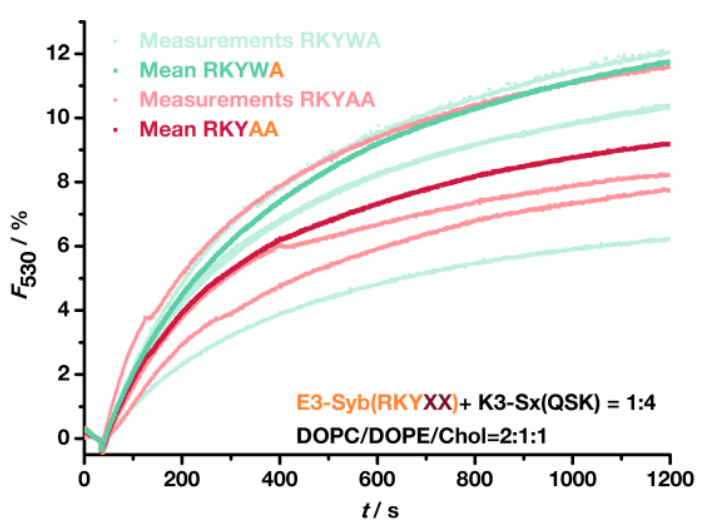

Additional data for Figure 5.4.

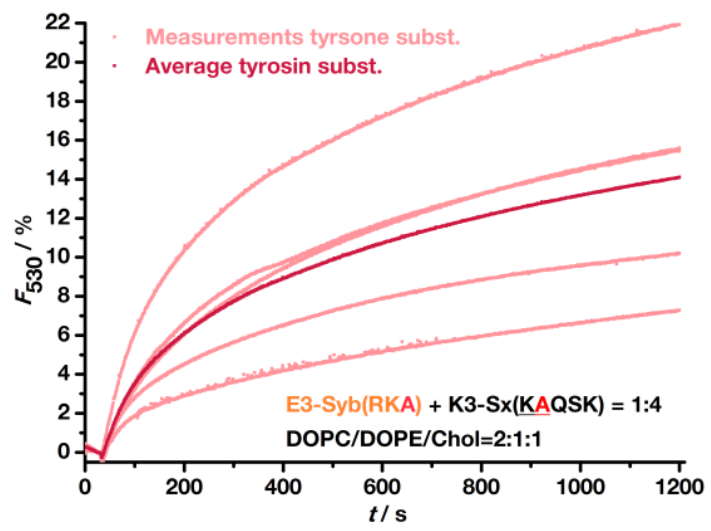

Additional data for Figure 5.6.

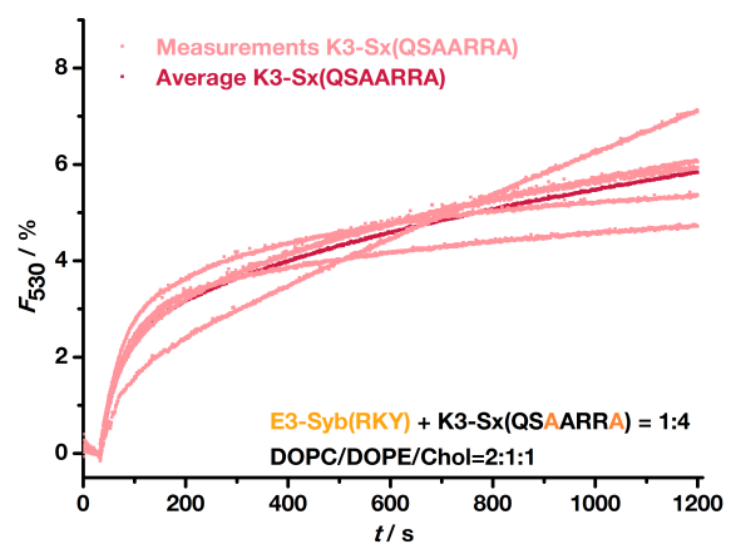

Additional data for Figure 5.10.

Figure A2: Individual fusion curves from FRET dequenching assays. The corresponding figures are indicated for each graph. 


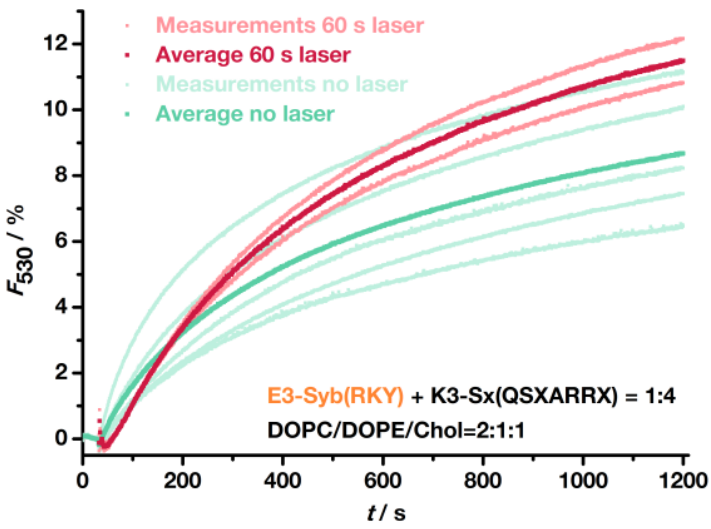

Additional data for Figure 6.9.

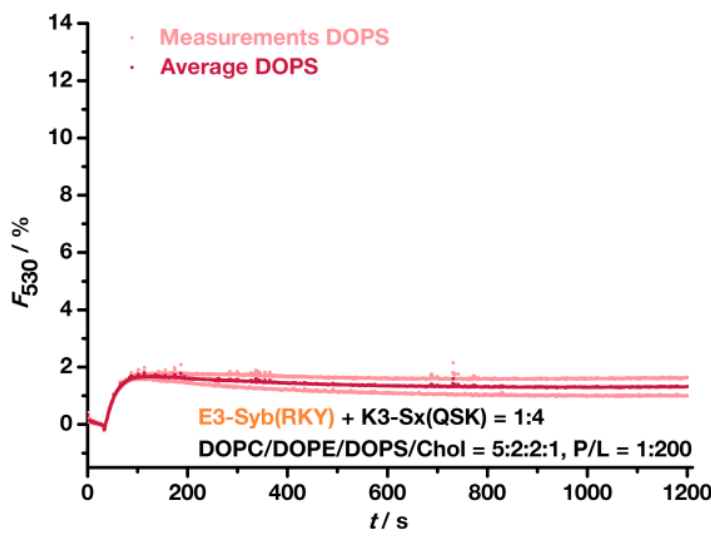

Additional data for Figure 7.2.

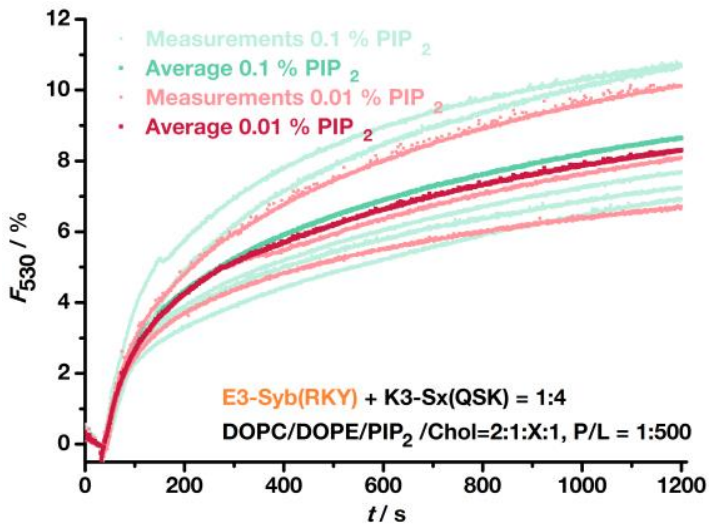

Additional data for Figure 7.4.

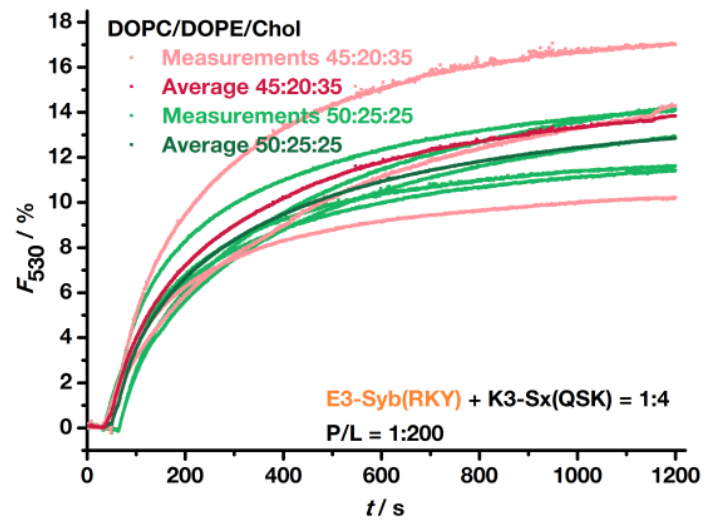

Additional data for Figure 7.1.

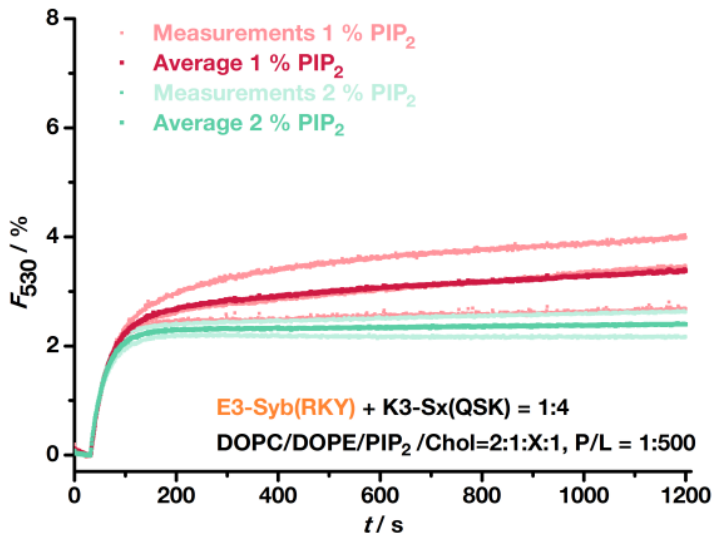

Additional data for Figure 7.3.

Figure A3: Individual fusion curves from FRET dequenching assays. The corresponding figures are indicated for each graph. 



\section{List of Abbreviations}

Amino acids are abbreviated using their standard three-letter or one-letter code.

\begin{tabular}{|c|c|}
\hline aa & amino acid \\
\hline aeg-PNA & $\mathrm{N}$-(2-aminoethyl)glycine-PNA \\
\hline AFF-1 & anchor-cell fusion failure 1 \\
\hline ATP & adenosine triphosphate \\
\hline AU & absorption unit \\
\hline $\mathrm{BD}$ & syringe syringe by Becton Dickinson \\
\hline Boc & butyloxycarbonyl protection group \\
\hline calc. & calculated \\
\hline Chol & cholesterol \\
\hline COVID-19 & coronavirus disease 2019 \\
\hline Cpx & complexins \\
\hline$\delta$ & chemical shift (NMR) \\
\hline DCM & dichloromethane \\
\hline DEACM & 7-diethylamino-4-(hydroxymethyl)coumarin \\
\hline DIC & $N, N$ '-diisopropylcarbodiimide \\
\hline DIPEA & $N, N$-diisopropylethylamine \\
\hline$d_{\mathrm{h}}$ & hydrodynamic diameter \\
\hline DLS & dynamic light scattering \\
\hline DMF & dimethylformamide \\
\hline DMSO & dimethylsulfoxide \\
\hline DNA & deoxyribonucleic acid \\
\hline DOPC & 1,2-dioleoyl-sn-glycero-3-phosphocholine \\
\hline DOPE & 1,2-dioleoyl-sn-glycero-3-phosphoethanolamine \\
\hline DOPS & 1,2-dioleoyl-sn-glycero-3-phospho-L-serine \\
\hline DTT & dithiothreitol \\
\hline$\varepsilon$ & extinction coefficient \\
\hline EDT & 1,2-ethanedithiole \\
\hline EDTA & ethylenediaminetetraacetic acid \\
\hline EFF-1 & epithelial fusion failure 1 \\
\hline eq. & equivalents \\
\hline EtOAC & ethyl acetate \\
\hline $\mathrm{EtOH}$ & ethanol \\
\hline ESI-MS & electrospray ionisation mass spectrometry \\
\hline$F_{530}$ & normalized fluorescence intensity at $530 \mathrm{~nm}$ \\
\hline
\end{tabular}




\begin{tabular}{|c|c|}
\hline FCCS & fluorescence cross-correlation spectroscopy \\
\hline Fmoc & 9-fluorenylmethoxycarbonyl protection group \\
\hline FRET & Förster resonance energy transfer \\
\hline GTP & guanosine tripshosphate \\
\hline HA & hemagglutinin \\
\hline HIV & human immunodeficiency viruses \\
\hline HEPES & 4-(2-hydroxyethyl)-1-piperazineethanesulfonic acid \\
\hline HFIP & 1,1,1,3,3,3-hexafluoro-2-propanol \\
\hline HPLC & high performance liquid chromatography \\
\hline HR-MS & high resolution mass spectrometry \\
\hline$J$ & coupling constant \\
\hline LPC & lysophosphatidylcholine \\
\hline LUV & large unilamellar vesicle \\
\hline$m / z$ & mass-to-charge ratio \\
\hline $\mathrm{MeCN}$ & acetonitrile \\
\hline $\mathrm{MeOH}$ & methanol \\
\hline MFG & mitofusin \\
\hline MLV & multilamellar vesicles \\
\hline Munc18 & mammalian uncoordinated-18 \\
\hline NBD & 7-nitro-2-1,3-benzoxadiazole \\
\hline NMR & nuclear magnetic resonance \\
\hline NSF & N-ethylmaleimide sensitive factor \\
\hline OG & Oregon Green 488 \\
\hline OPa1 & optic atrophy protein 1 \\
\hline Oxyma & ethyl cyanohydroxyiminoacetate \\
\hline $\mathrm{Pbf}$ & 2,2,4,6,7-pentamethyldihydrobenzofuran-5-sulfonyl \\
\hline $\mathrm{PC}$ & phosphatidylcholine \\
\hline PE & phosphatidylethanolamine \\
\hline PEG & polyethylene glycol \\
\hline $\mathrm{PIP}_{2}$ & phosphatidylinositol 4,5-bisphosphate \\
\hline $\mathrm{P} / \mathrm{L}$ ratio & peptide-to-lipid ratio \\
\hline PNA & PNA peptide nucleic acid \\
\hline POPC & 1-palmitoyl-2-oleoyl-sn-glycero-3-phosphocholine \\
\hline ppm & ppm parts per million \\
\hline $\mathrm{Rh}$ & Lissamine Rhodamine B \\
\hline $\mathrm{RP}$ & reversed phase \\
\hline $\mathrm{rpm}$ & rotations per minute \\
\hline RT & room temperature \\
\hline $\mathrm{R}_{\mathrm{t}}$ & retention time \\
\hline SARS-CoV-2 & severe acute respiratory syndrome coronavirus 2 \\
\hline SM & Sec1/Munc18-like \\
\hline SNAP & soluble NSF attachment protein \\
\hline SNAP-25 & 25-kDa synaptosome-associated protein \\
\hline
\end{tabular}


SNARE

SPPS

SUV

Sx

Syb

Syt1

${ }^{t} \mathrm{Bu}$

TFA

TFE

TIS

THF

TLC

TMD

TR

Trt

UPLC

UV

$v / v$ soluble $N$-ethylmaleimide-sensitive factor attachment protein receptor SPPS solid phase peptide synthesis

small unilamellar vesicle

Syntaxin 1A

synaptobrevin 2

synaptotagmin 1

tert-butyl protection group

trifluoroacetic acid

trifluoroethanol

triisopropylsilane

tetrahydrofuran

thin layer chromatography

transmembrane domain

Texas Red

trityl protection group

ultra-performance liquid chromatography

ultraviolet

volume/volume ratio 



\section{Bibliography}

[1] T. C. Südhof, Neurotransmitter release: the last millisecond in the life of a synaptic vesicle, Neuron 2013, 80, 675.

[2] T. C. Südhof, The synaptic vesicle cycle, Annu Rev Neurosci 2004, 27, 509.

[3] W. Antonin, D. Fasshauer, S. Becker, R. Jahn, T. R. Schneider, Crystal structure of the endosomal SNARE complex reveals common structural principles of all SNAREs, Nat Struct Biol 2002, 9, 107.

[4] R. W. Baker, F. M. Hughson, Chaperoning SNARE assembly and disassembly, Nat Rev Mol Cell Biol 2016, 17, 465.

[5] Y. A. Chen, R. H. Scheller, SNARE-mediated membrane fusion, Nat Rev Mol Cell Biol 2001, 2, 98 .

[6] R. Jahn, R. H. Scheller, SNAREs--engines for membrane fusion, Nat Rev Mol Cell Biol 2006, 7, 631.

[7] A. V. Pobbati, A. Stein, D. Fasshauer, N- to C-terminal SNARE complex assembly promotes rapid membrane fusion, Science 2006, 313, 673.

[8] X. Lou, Y.-K. Shin, SNARE zippering, Biosci Rep 2016, 36.

[9] Y. Gao, S. Zorman, G. Gundersen, Z. Xi, L. Ma, G. Sirinakis, J. E. Rothman, Y. Zhang, Single reconstituted neuronal SNARE complexes zipper in three distinct stages, Science 2012, 337, 1340.

[10] R. B. Sutton, D. Fasshauer, R. Jahn, A. T. Brunger, Crystal structure of a SNARE complex involved in synaptic exocytosis at 2.4 A resolution, Nature 1998, 395, 347.

[11] M. A. Poirier, W. Xiao, J. C. Macosko, C. Chan, Y. K. Shin, M. K. Bennett, The synaptic SNARE complex is a parallel four-stranded helical bundle, Nat Struct Biol 1998, $5,765$.

[12] D. Fasshauer, Structural insights into the SNARE mechanism, Biochim Biophys Acta Mol Cell Res 2003, 1641, 87.

[13] W. Hong, SNAREs and traffic, Biochim Biophys Acta 2005, 1744, 120.

[14] W. S. Trimble, D. M. Cowan, R. H. Scheller, VAMP-1: a synaptic vesicleassociated integral membrane protein, Proc Natl Acad Sci U S A 1988, 85, 4538. 
[15] M. Baumert, P. R. Maycox, F. Navone, P. de Camilli, R. Jahn, Synaptobrevin: an integral membrane protein of 18,000 daltons present in small synaptic vesicles of rat brain, EMBO J 1989, 8, 379.

[16] M. K. Bennett, N. Calakos, R. H. Scheller, Syntaxin: a synaptic protein implicated in docking of synaptic vesicles at presynaptic active zones, Science 1992, 257, 255.

[17] P. Kumar, S. Guha, U. Diederichsen, SNARE protein analog-mediated membrane fusion, J. Pept. Sci. 2015, 21, 621.

[18] A. Kashiwada, M. Tsuboi, K. Matsuda, Target-selective vesicle fusion induced by molecular recognition on lipid bilayers, Chem. Commun. 2009, 695.

[19] H. Robson Marsden, N. A. Elbers, P. H. H. Bomans, N. A. J. M. Sommerdijk, A. Kros, A reduced SNARE model for membrane fusion, Angew. Chem. Int. Ed. 2009, $48,2330$.

[20] G. Stengel, R. Zahn, F. Höök, DNA-induced programmable fusion of phospholipid vesicles, J Am Chem Soc 2007, 129, 9584.

[21] J. R. Litowski, R. S. Hodges, Designing Heterodimeric Two-stranded $\alpha$-Helical Coiled-coils, J Biol Chem 2002, 277, 37272.

[22] K. Meyenberg, A. S. Lygina, G. van den Bogaart, R. Jahn, U. Diederichsen, SNARE derived peptide mimic inducing membrane fusion, Chem. Commun. 2011, 47, 9405.

[23] A. Stein, G. Weber, M. C. Wahl, R. Jahn, Helical extension of the neuronal SNARE complex into the membrane, Nature 2009, 460, 525.

[24] D. Singer-Lahat, N. Barak-Broner, A. Sheinin, D. Greitzer-Antes, I. Michaelevski, I. Lotan, The Dual Function of the Polybasic Juxtamembrane Region of Syntaxin 1A in Clamping Spontaneous Release and Stimulating Ca2+-Triggered Release in Neuroendocrine Cells, J Neurosci 2018, 38, 220.

[25] J. S. van Komen, X. Bai, T. L. Rodkey, J. Schaub, J. A. McNew, The polybasic juxtamembrane region of Sso1p is required for SNARE function in vivo, Eukaryotic Cell 2005, 4, 2017.

[26] D. H. Murray, L. K. Tamm, Clustering of syntaxin-1A in model membranes is modulated by phosphatidylinositol 4,5-bisphosphate and cholesterol, Biochemistry 2009, 48, 4617.

[27] K. Aoyagi, T. Sugaya, M. Umeda, S. Yamamoto, S. Terakawa, M. Takahashi, The activation of exocytotic sites by the formation of phosphatidylinositol 4,5bisphosphate microdomains at syntaxin clusters, J Biol Chem 2005, 280, 17346.

[28] D. J. James, C. Khodthong, J. A. Kowalchyk, T. F. J. Martin, Phosphatidylinositol 4,5-bisphosphate regulates SNARE-dependent membrane fusion, J Cell Biol 2008, 182,355 . 
[29] M. Borisovska, Y. N. Schwarz, M. Dhara, A. Yarzagaray, S. Hugo, D. Narzi, S. W. I. Siu, J. Kesavan, R. Mohrmann, R. A. Böckmann et al., Membrane-proximal tryptophans of synaptobrevin II stabilize priming of secretory vesicles, J Neurosci 2012, 32, 15983.

[30] Q. Fang, Y. Zhao, M. Lindau, Juxtamembrane tryptophans of synaptobrevin 2 control the process of membrane fusion, FEBS Lett 2013, 587, 67.

[31] M. S. Bretscher, M. C. Raff, Mammalian plasma membranes, Nature 1975, 258, 43.

[32] G. van Meer, D. R. Voelker, G. W. Feigenson, Membrane lipids: where they are and how they behave, Nat Rev Mol Cell Biol 2008, 9, 112.

[33] M. Cai, J. Gao, H. Wang (Eds.) Composition and Function of Cell Membranes, Springer Nature, Singapore, 2018.

[34] C. Tanford, The hydrophobic effect and the organization of living matter, Science 1978, 200, 1012.

[35] G. L. Nicolson, The Fluid-Mosaic Model of Membrane Structure: still relevant to understanding the structure, function and dynamics of biological membranes after more than 40 years, Biochim Biophys Acta 2014, 1838, 1451.

[36] G. A. Jamieson, D. M. Robinson, Mammalian Cell Membranes. Volume 2: The Diversity of Membranes, Elsevier Science, Burlington, 1977.

[37] D. J. Müller, N. Wu, K. Palczewski, Vertebrate membrane proteins: structure, function, and insights from biophysical approaches, Pharmacol Rev 2008, 60, 43.

[38] Z. Cournia, T. W. Allen, I. Andricioaei, B. Antonny, D. Baum, G. Brannigan, N.-V. Buchete, J. T. Deckman, L. Delemotte, C. Del Val et al., Membrane Protein Structure, Function, and Dynamics: A Perspective from Experiments and Theory, $J$ Membr Biol 2015, 248, 611.

[39] L. E. Hedin, K. Illergård, A. Elofsson, An introduction to membrane proteins, $J$ Proteome Res 2011, 10, 3324.

[40] J. W. Fairman, N. Noinaj, S. K. Buchanan, The structural biology of $\beta$-barrel membrane proteins: a summary of recent reports, Curr Opin Struct Biol 2011, 21, 523.

[41] V. Monje-Galvan, J. B. Klauda, Peripheral membrane proteins: Tying the knot between experiment and computation, Biochim Biophys Acta 2016, 1858, 1584.

[42] B. A. Seaton, M. F. Roberts in Biological membranes. A molecular perspective from computation and experiment (Eds.: K. M. Merz, B. Roux), Birkhäuser Boston, Boston, 1996, pp. 355-403.

[43] J. M. Crane, V. Kiessling, L. K. Tamm, Measuring lipid asymmetry in planar supported bilayers by fluorescence interference contrast microscopy, Langmuir 2005, 21, 1377. 
[44] M. S. Bretscher, Asymmetrical lipid bilayer structure for biological membranes, Nature New Biol 1972, 236, 11.

[45] J. M. Boon, B. D. Smith, Chemical control of phospholipid distribution across bilayer membranes, Med Res Rev 2002, 22, 251.

[46] S. J. Singer, G. L. Nicolson, The fluid mosaic model of the structure of cell membranes, Science 1972, 175, 720.

[47] J. F. Danielli, H. Davson, A contribution to the theory of permeability of thin films, J. Cell. Comp. Physiol. 1935, 5, 495.

[48] J. D. ROBERTSON, The ultrastructure of cell membranes and their derivatives, Biochem Soc Symp 1959, 16, 3.

[49] J. D. ROBERTSON, The molecular structure and contact relationships of cell membranes, Prog Biophys Mol Biol 1960, 10, 343.

[50] K. Simons, E. Ikonen, Functional rafts in cell membranes, Nature 1997, 387, 569.

[51] R. Varma, S. Mayor, GPI-anchored proteins are organized in submicron domains at the cell surface, Nature 1998, 394, 798.

[52] E. Sezgin, I. Levental, S. Mayor, C. Eggeling, The mystery of membrane organization: composition, regulation and roles of lipid rafts, Nat Rev Mol Cell Biol 2017, 18,361 .

[53] E. J. Shimshick, H. M. McConnell, Lateral phase separation in phospholipid membranes, Biochemistry 1973, 12, 2351.

[54] L. J. Pike, Rafts defined: a report on the Keystone Symposium on Lipid Rafts and Cell Function, J Lipid Res 2006, 47, 1597.

[55] F. M. Goñi, "Rafts": A nickname for putative transient nanodomains, Chem Phys Lipids 2019, 218, 34.

[56] M. L. Kraft, Plasma membrane organization and function: moving past lipid rafts, Mol Biol Cell 2013, 24, 2765.

[57] T. Yeung, G. E. Gilbert, J. Shi, J. Silvius, A. Kapus, S. Grinstein, Membrane phosphatidylserine regulates surface charge and protein localization, Science $\mathbf{2 0 0 8}$, $319,210$.

[58] E. Sevcsik, G. J. Schütz, With or without rafts? Alternative views on cell membranes, Bioessays 2016, 38, 129.

[59] R. G. W. Anderson, K. Jacobson, A role for lipid shells in targeting proteins to caveolae, rafts, and other lipid domains, Science 2002, 296, 1821.

[60] L. Lim, M. R. Wenk in Handbook of Neurochemistry and Molecular Neurobiology. Neural Lipids (Eds.: A. Lajtha, G. Goracci, G. Tettamanti), Springer US, Boston, MA, 2009, pp. 223-238. 
[61] T. Harayama, H. Riezman, Understanding the diversity of membrane lipid composition, Nat Rev Mol Cell Biol 2018, 19, 281.

[62] O. G. Mouritsen, The liquid-ordered state comes of age, Biochim Biophys Acta 2010, 1798, 1286.

[63] M. Cebecauer, M. Amaro, P. Jurkiewicz, M. J. Sarmento, R. Šachl, L. Cwiklik, M. Hof, Membrane Lipid Nanodomains, Chem Rev 2018, 118, 11259.

[64] O. S. Andersen, R. E. Koeppe, Bilayer thickness and membrane protein function: an energetic perspective, Annu Rev Biophys Biomol Struct 2007, 36, 107.

[65] S.-T. Yang, A. J. B. Kreutzberger, J. Lee, V. Kiessling, L. K. Tamm, The role of cholesterol in membrane fusion, Chem Phys Lipids 2016, 199, 136.

[66] L. Bagatolli, P. B. Sunil Kumar, Phase behavior of multicomponent membranes: Experimental and computational techniques, Soft Matter 2009, 5, 3234.

[67] H. T. McMahon, E. Boucrot, Membrane curvature at a glance, J Cell Sci 2015, 128, 1065.

[68] A. M. Smondyrev, M. L. Berkowitz, Structure of Dipalmitoylphosphatidylcholine/ Cholesterol Bilayer at Low and High Cholesterol Concentrations: Molecular Dynamics Simulation, Biophys J 1999, 77, 2075.

[69] P. Heftberger, B. Kollmitzer, A. A. Rieder, H. Amenitsch, G. Pabst, In situ determination of structure and fluctuations of coexisting fluid membrane domains, Biophys J 2015, 108, 854.

[70] J. J. Hulce, A. B. Cognetta, M. J. Niphakis, S. E. Tully, B. F. Cravatt, Proteomewide mapping of cholesterol-interacting proteins in mammalian cells, Nat Methods 2013, 10, 259 .

[71] J. Fantini, F. J. Barrantes, How cholesterol interacts with membrane proteins: an exploration of cholesterol-binding sites including CRAC, CARC, and tilted domains, Front Physiol 2013, 4, 31 .

[72] M. M. Kozlov, V. S. Markin, Vozmozhnyĭ mekhanizm sliiania membran, Biofizika 1983, 28, 242.

[73] L. V. Chernomordik, M. M. Kozlov, Membrane hemifusion: crossing a chasm in two leaps, Cell 2005, 123, 375.

[74] P. M. Kasson, N. W. Kelley, N. Singhal, M. Vrljic, A. T. Brunger, V. S. Pande, Ensemble molecular dynamics yields submillisecond kinetics and intermediates of membrane fusion, Proc Natl Acad Sci U S A 2006, 103, 11916.

[75] L. Yang, H. W. Huang, Observation of a membrane fusion intermediate structure, Science 2002, 297, 1877. 
[76] S. Aeffner, T. Reusch, B. Weinhausen, T. Salditt, Energetics of stalk intermediates in membrane fusion are controlled by lipid composition, Proc Natl Acad Sci U S A 2012, 109, E1609-18.

[77] J. M. Hernandez, A. Stein, E. Behrmann, D. Riedel, A. Cypionka, Z. Farsi, P. J. Walla, S. Raunser, R. Jahn, Membrane fusion intermediates via directional and full assembly of the SNARE complex, Science 2012, 336, 1581.

[78] J. Nikolaus, M. Stöckl, D. Langosch, R. Volkmer, A. Herrmann, Direct visualization of large and protein-free hemifusion diaphragms, Biophys J 2010, 98, 1192.

[79] R. J. Ryham, T. S. Klotz, L. Yao, F. S. Cohen, Calculating Transition Energy Barriers and Characterizing Activation States for Steps of Fusion, Biophys J 2016, $110,1110$.

[80] Y. Kozlovsky, M. M. Kozlov, Stalk Model of Membrane Fusion: Solution of Energy Crisis, Biophys J 2002, 82, 882.

[81] D. P. Siegel, Energetics of intermediates in membrane fusion: comparison of stalk and inverted micellar intermediate mechanisms, Biophys $J$ 1993, 65, 2124.

[82] P. I. Kuzmin, J. Zimmerberg, Y. A. Chizmadzhev, F. S. Cohen, A quantitative model for membrane fusion based on low-energy intermediates, Proc Natl Acad Sci U S A 2001, 98, 7235.

[83] M. Fuhrmans, G. Marelli, Y. G. Smirnova, M. Müller, Mechanics of membrane fusion/pore formation, Chem Phys Lipids 2015, 185, 109.

[84] M. Müller, K. Katsov, M. Schick, New mechanism of membrane fusion, J. Chem. Phys. 2002, 116, 2342.

[85] H. J. Risselada, G. Marelli, M. Fuhrmans, Y. G. Smirnova, H. Grubmüller, S. J. Marrink, M. Müller, Line-tension controlled mechanism for influenza fusion, PLoS One 2012, 7, e38302.

[86] A. F. Smeijers, A. J. Markvoort, K. Pieterse, P. A. J. Hilbers, A detailed look at vesicle fusion, J Phys Chem B 2006, 110, 13212.

[87] K. Katsov, M. Müller, M. Schick, Field theoretic study of bilayer membrane fusion: II. Mechanism of a stalk-hole complex, Biophys $J$ 2006, 90, 915.

[88] M. Müller, K. Katsov, M. Schick, A New Mechanism of Model Membrane Fusion Determined from Monte Carlo Simulation, Biophys J 2003, 85, 1611.

[89] S. A. Akimov, R. J. Molotkovsky, P. I. Kuzmin, T. R. Galimzyanov, O. V. Batishchev, Continuum Models of Membrane Fusion: Evolution of the Theory, Int J Mol Sci 2020, 21.

[90] A. J. Markvoort, S. J. Marrink, Lipid acrobatics in the membrane fusion arena, Curr Top Membr 2011, 68, 259. 
[91] L. V. Chernomordik, M. M. Kozlov, Mechanics of membrane fusion, Nat Struct Mol Biol 2008, 15, 675 .

[92] B. Katz, R. Miledi, The timing of calcium action during neuromuscular transmission, J Physiol (Lond) 1967, 189, 535.

[93] S. SANKARANARAYANAN, T. A. RYAN in Protein trafficking in neurons (Ed.: A. J. Bean), Elsevier/Academic Press, Amsterdam, Boston, 2007, pp. 97-124.

[94] R. Jahn, D. Fasshauer, Molecular machines governing exocytosis of synaptic vesicles, Nature 2012, 490, 201.

[95] P. S. Kaeser, W. G. Regehr, The readily releasable pool of synaptic vesicles, Curr Opin Neurobiol 2017, 43, 63.

[96] S. C. Harrison, Viral membrane fusion, Virology 2015, 479-480, 498.

[97] T. Tang, M. Bidon, J. A. Jaimes, G. R. Whittaker, S. Daniel, Coronavirus membrane fusion mechanism offers a potential target for antiviral development, Antiviral Res 2020, 178, 104792.

[98] S. Martens, H. T. McMahon, Mechanisms of membrane fusion: disparate players and common principles, Nat Rev Mol Cell Biol 2008, 9, 543.

[99] J. M. White, S. E. Delos, M. Brecher, K. Schornberg, Structures and mechanisms of viral membrane fusion proteins: multiple variations on a common theme, Crit Rev Biochem Mol Biol 2008, 43, 189.

[100] I. A. Wilson, J. J. Skehel, D. C. Wiley, Structure of the haemagglutinin membrane glycoprotein of influenza virus at 3 A resolution, Nature 1981, 289, 366.

[101] J. J. Skehel, D. C. Wiley, Receptor binding and membrane fusion in virus entry: the influenza hemagglutinin, Annu Rev Biochem 2000, 69, 531.

[102] M. Kielian, Mechanisms of Virus Membrane Fusion Proteins, Annu Rev Virol 2014, $1,171$.

[103] J. M. White, G. R. Whittaker, Fusion of Enveloped Viruses in Endosomes, Traffic 2016, 17, 593.

[104] L. Wang, Y. Wang, D. Ye, Q. Liu, Review of the 2019 novel coronavirus (SARSCoV-2) based on current evidence, Int J Antimicrob Agents 2020, 55, 105948.

[105] S. Belouzard, J. K. Millet, B. N. Licitra, G. R. Whittaker, Mechanisms of coronavirus cell entry mediated by the viral spike protein, Viruses 2012, 4, 1011.

[106] Y. Yuan, D. Cao, Y. Zhang, J. Ma, J. Qi, Q. Wang, G. Lu, Y. Wu, J. Yan, Y. Shi et al., Cryo-EM structures of MERS-CoV and SARS-CoV spike glycoproteins reveal the dynamic receptor binding domains, Nat Commun 2017, 8, 15092. 
[107] B. J. Bosch, R. van der Zee, C. A. M. de Haan, P. J. M. Rottier, The coronavirus spike protein is a class I virus fusion protein: structural and functional characterization of the fusion core complex, J Virol 2003, 77, 8801.

[108] G. Simmons, D. N. Gosalia, A. J. Rennekamp, J. D. Reeves, S. L. Diamond, P. Bates, Inhibitors of cathepsin L prevent severe acute respiratory syndrome coronavirus entry, Proc Natl Acad Sci U S A 2005, 102, 11876.

[109] G. Simmons, J. D. Reeves, A. J. Rennekamp, S. M. Amberg, A. J. Piefer, P. Bates, Characterization of severe acute respiratory syndrome-associated coronavirus (SARS-CoV) spike glycoprotein-mediated viral entry, Proc Natl Acad Sci U S A 2004, 101, 4240.

[110] M. Giacomello, A. Pyakurel, C. Glytsou, L. Scorrano, The cell biology of mitochondrial membrane dynamics, Nat Rev Mol Cell Biol 2020, 21, 204.

[111] L. Tilokani, S. Nagashima, V. Paupe, J. Prudent, Mitochondrial dynamics: overview of molecular mechanisms, Essays Biochem 2018, 62, 341.

[112] A. M. van der Bliek, Q. Shen, S. Kawajiri, Mechanisms of mitochondrial fission and fusion, Cold Spring Harb Perspect Biol 2013, 5.

[113] T. "Brandt, L. Cavellini, W. Kühlbrandt, M. M. Cohen, A mitofusin-dependent docking ring complex triggers mitochondrial fusion in vitro, Elife 2016, 5.

[114] E. H. Chen, E. Grote, W. Mohler, A. Vignery, Cell-cell fusion, FEBS Lett 2007, $581,2181$.

[115] N. Takahashi, Y. Kobayashi, N. Udagawa in Principles of bone biology (Ed.: J. P. Bilezikian), Acad. Press, San Diego [u.a.], 2020, pp. 111-131.

[116] V. Horsley, G. K. Pavlath, Forming a multinucleated cell: molecules that regulate myoblast fusion, Cells Tissues Organs (Print) 2004, 176, 67.

[117] M. J. Petrany, D. P. Millay, Cell Fusion: Merging Membranes and Making Muscle, Trends Cell Biol 2019, 29, 964.

[118] J. Pérez-Vargas, T. Krey, C. Valansi, O. Avinoam, A. Haouz, M. Jamin, H. RavehBarak, B. Podbilewicz, F. A. Rey, Structural basis of eukaryotic cell-cell fusion, Cell 2014, 157, 407.

[119] B. Podbilewicz, E. Leikina, A. Sapir, C. Valansi, M. Suissa, G. Shemer, L. V. Chernomordik, The C. elegans developmental fusogen EFF-1 mediates homotypic fusion in heterologous cells and in vivo, Dev Cell 2006, 11, 471.

[120] A. Sapir, J. Choi, E. Leikina, O. Avinoam, C. Valansi, L. V. Chernomordik, A. P. Newman, B. Podbilewicz, AFF-1, a FOS-1-regulated fusogen, mediates fusion of the anchor cell in C. elegans, Dev Cell 2007, 12, 683. 
[121] E. F. Pettersen, T. D. Goddard, C. C. Huang, G. S. Couch, D. M. Greenblatt, E. C. Meng, T. E. Ferrin, UCSF Chimera--a visualization system for exploratory research and analysis, J Comput Chem 2004, 25, 1605.

[122] D. Fasshauer, R. B. Sutton, A. T. Brunger, R. Jahn, Conserved structural features of the synaptic fusion complex: SNARE proteins reclassified as Q- and R-SNAREs, Proc Natl Acad Sci U S A 1998, 95, 15781.

[123] D. Fasshauer, W. Antonin, V. Subramaniam, R. Jahn, SNARE assembly and disassembly exhibit a pronounced hysteresis, Nat Struct Biol 2002, 9, 144.

[124] J.-K. Ryu, R. Jahn, T.-Y. Yoon, Review: Progresses in understanding Nethylmaleimide sensitive factor (NSF) mediated disassembly of SNARE complexes, Biopolymers 2016, 105, 518.

[125] K. Wiederhold, D. Fasshauer, Is assembly of the SNARE complex enough to fuel membrane fusion?, J. Biol. Chem. 2009, 284, 13143.

[126] X. Lu, Y. Zhang, Y.-K. Shin, Supramolecular SNARE assembly precedes hemifusion in SNARE-mediated membrane fusion, Nat Struct Mol Biol 2008, 15, 700 .

[127] H. J. Risselada, C. Kutzner, H. Grubmüller, Caught in the act: visualization of SNARE-mediated fusion events in molecular detail, Chembiochem 2011, 12, 1049.

[128] V. Knecht, H. Grubmüller, Mechanical Coupling via the Membrane Fusion SNARE Protein Syntaxin 1A: A Molecular Dynamics Study, Biophys J 2003, 84, 1527.

[129] J. Han, K. Pluhackova, D. Bruns, R. A. Böckmann, Synaptobrevin transmembrane domain determines the structure and dynamics of the SNARE motif and the linker region, Biochim Biophys Acta 2016, 1858, 855.

[130] A. E. Blanchard, M. J. Arcario, K. Schulten, E. Tajkhorshid, A highly tilted membrane configuration for the prefusion state of synaptobrevin, Biophys $J$ 2014, $107,2112$.

[131] L. E. Dietrich, C. Boeddinghaus, T. J. LaGrassa, C. Ungermann, Control of eukaryotic membrane fusion by N-terminal domains of SNARE proteins, Biochim Biophys Acta Mol Cell Res 2003, 1641, 111.

[132] M. Margittai, J. Widengren, E. Schweinberger, G. F. Schröder, S. Felekyan, E. Haustein, M. König, D. Fasshauer, H. Grubmüller, R. Jahn et al., Single-molecule fluorescence resonance energy transfer reveals a dynamic equilibrium between closed and open conformations of syntaxin 1, Proc Natl Acad Sci U S A 2003, 100, 15516.

[133] J. Rizo, T. C. Südhof, Snares and Munc18 in synaptic vesicle fusion, Nat Rev Neurosci 2002, 3, 641 . 
[134] J. S. van Komen, X. Bai, B. L. Scott, J. A. McNew, An intramolecular t-SNARE complex functions in vivo without the syntaxin NH2-terminal regulatory domain, $J$ Cell Biol 2006, 172, 295.

[135] Y. Wang, I. Dulubova, J. Rizo, T. C. Südhof, Functional analysis of conserved structural elements in yeast syntaxin Vam3p, J. Biol. Chem. 2001, 276, 28598.

[136] R. F. Toonen, M. Verhage, Vesicle trafficking: pleasure and pain from SM genes, Trends Cell Biol 2003, 13, 177.

[137] F. Li, F. Pincet, E. Perez, W. S. Eng, T. J. Melia, J. E. Rothman, D. Tareste, Energetics and dynamics of SNAREpin folding across lipid bilayers, Nat Struct Mol Biol 2007, 14, 890.

[138] L. Ma, Y. Kang, J. Jiao, A. A. Rebane, H. K. Cha, Z. Xi, H. Qu, Y. Zhang, $\alpha$-SNAP Enhances SNARE Zippering by Stabilizing the SNARE Four-Helix Bundle, Cell Rep 2016, 15, 531.

[139] F. Li, N. Tiwari, J. E. Rothman, F. Pincet, Kinetic barriers to SNAREpin assembly in the regulation of membrane docking/priming and fusion, Proc Natl Acad Sci U S A 2016, 113, 10536.

[140] J. Shin, X. Lou, D.-H. Kweon, Y.-K. Shin, Multiple conformations of a single SNAREpin between two nanodisc membranes reveal diverse pre-fusion states, Biochem J 2014, 459, 95.

[141] D. Min, K. Kim, C. Hyeon, Y. H. Cho, Y.-K. Shin, T.-Y. Yoon, Mechanical unzipping and rezipping of a single SNARE complex reveals hysteresis as a forcegenerating mechanism, Nat Commun 2013, 4, 1705.

[142] J. Wang, O. Bello, S. M. Auclair, J. Coleman, F. Pincet, S. S. Krishnakumar, C. V. Sindelar, J. E. Rothman, Calcium sensitive ring-like oligomers formed by synaptotagmin, Proc Natl Acad Sci U S A 2014, 111, 13966.

[143] D. Kümmel, S. S. Krishnakumar, D. T. Radoff, F. Li, C. G. Giraudo, F. Pincet, J. E. Rothman, K. M. Reinisch, Complexin cross-links prefusion SNAREs into a zigzag array, Nat Struct Mol Biol 2011, 18, 927.

[144] Q. Fang, M. Lindau, How could SNARE proteins open a fusion pore?, Physiology (Bethesda) 2014, 29, 278.

[145] A. N. Ngatchou, K. Kisler, Q. Fang, A. M. Walter, Y. Zhao, D. Bruns, J. B. Sørensen, M. Lindau, Role of the synaptobrevin $\mathrm{C}$ terminus in fusion pore formation, Proc Natl Acad Sci U S A 2010, 107, 18463.

[146] F. Li, D. Kümmel, J. Coleman, K. M. Reinisch, J. E. Rothman, F. Pincet, A halfzippered SNARE complex represents a functional intermediate in membrane fusion, J Am Chem Soc 2014, 136, 3456. 
[147] A. Witkowska, R. Jahn, Rapid SNARE-Mediated Fusion of Liposomes and Chromaffin Granules with Giant Unilamellar Vesicles, Biophys J 2017, 113, 1251.

[148] T. C. Südhof, A molecular machine for neurotransmitter release: synaptotagmin and beyond, Nat Med 2013, 19, 1227.

[149] S. Wang, Y. Li, J. Gong, S. Ye, X. Yang, R. Zhang, C. Ma, Munc18 and Munc13 serve as a functional template to orchestrate neuronal SNARE complex assembly, Nat Commun 2019, 10, 69.

[150] T. C. Südhof, J. E. Rothman, Membrane fusion: grappling with SNARE and SM proteins, Science 2009, 323, 474.

[151] J. E. Gerst, SNARE regulators: matchmakers and matchbreakers, Biochim Biophys Acta Mol Cell Res 2003, 1641, 99.

[152] J. Rizo, Mechanism of neurotransmitter release coming into focus, Protein Sci 2018 , $27,1364$.

[153] K. M. Misura, R. H. Scheller, W. I. Weis, Three-dimensional structure of the neuronal-Sec1-syntaxin 1a complex, Nature 2000, 404, 355.

[154] J. Rizo, J. Xu, The Synaptic Vesicle Release Machinery, Annu Rev Biophys 2015, $44,339$.

[155] E. He, K. Wierda, R. van Westen, J. H. Broeke, R. F. Toonen, L. N. Cornelisse, M. Verhage, Munc13-1 and Munc18-1 together prevent NSF-dependent de-priming of synaptic vesicles, Nat Commun 2017, 8, 15915.

[156] M. Khvotchev, I. Dulubova, J. Sun, H. Dai, J. Rizo, T. C. Südhof, Dual modes of Munc18-1/SNARE interactions are coupled by functionally critical binding to syntaxin-1 N terminus, J Neurosci 2007, 27, 12147.

[157] C. Ma, L. Su, A. B. Seven, Y. Xu, J. Rizo, Reconstitution of the vital functions of Munc18 and Munc13 in neurotransmitter release, Science 2013, 339, 421.

[158] X. Yang, S. Wang, Y. Sheng, M. Zhang, W. Zou, L. Wu, L. Kang, J. Rizo, R. Zhang, T. Xu et al., Syntaxin opening by the MUN domain underlies the function of Munc13 in synaptic-vesicle priming, Nat Struct Mol Biol 2015, 22, 547.

[159] Y. Lai, U. B. Choi, J. Leitz, H. J. Rhee, C. Lee, B. Altas, M. Zhao, R. A. Pfuetzner, A. L. Wang, N. Brose et al., Molecular Mechanisms of Synaptic Vesicle Priming by Munc13 and Munc18, Neuron 2017, 95, 591-607.e10.

[160] E. R. Chapman, How does synaptotagmin trigger neurotransmitter release?, Аnnu Rev Biochem 2008, 77, 615.

[161] K. L. Fuson, M. Montes, J. J. Robert, R. B. Sutton, Structure of human synaptotagmin $1 \mathrm{C} 2 \mathrm{AB}$ in the absence of $\mathrm{Ca} 2+$ reveals a novel domain association, Biochemistry 2007, 46, 13041. 
[162] W. C. Tucker, T. Weber, E. R. Chapman, Reconstitution of Ca2+-regulated membrane fusion by synaptotagmin and SNAREs, Science 2004, 304, 435.

[163] K. Reim, M. Mansour, F. Varoqueaux, H. T. McMahon, T. C. Südhof, N. Brose, C. Rosenmund, Complexins Regulate a Late Step in Ca2+-Dependent Neurotransmitter Release, Cell 2001, 104, 71.

[164] J. Tang, A. Maximov, O.-H. Shin, H. Dai, J. Rizo, T. C. Südhof, A complexin/synaptotagmin 1 switch controls fast synaptic vesicle exocytosis, Cell 2006, 126, 1175 .

[165] N. A. Courtney, H. Bao, J. S. Briguglio, E. R. Chapman, Synaptotagmin 1 clamps synaptic vesicle fusion in mammalian neurons independent of complexin, Nat Commun 2019, 10, 4076.

[166] Q. Zhou, P. Zhou, A. L. Wang, D. Wu, M. Zhao, T. C. Südhof, A. T. Brunger, The primed SNARE-complexin-synaptotagmin complex for neuronal exocytosis, Nature 2017, 548, 420.

[167] M. Zhao, S. Wu, Q. Zhou, S. Vivona, D. J. Cipriano, Y. Cheng, A. T. Brunger, Mechanistic insights into the recycling machine of the SNARE complex, Nature 2015, 518, 61 .

[168] J. Han, K. Pluhackova, R. A. Böckmann, The Multifaceted Role of SNARE Proteins in Membrane Fusion, Front Physiol 2017, 8, 5.

[169] M. S. Al-Abdul-Wahid, C. M. DeMill, M. B. Serwin, R. S. Prosser, B. A. Stewart, Effect of juxtamembrane tryptophans on the immersion depth of Synaptobrevin, an integral vesicle membrane protein, Biochim Biophys Acta 2012, 1818, 2994.

[170] J. Kesavan, M. Borisovska, D. Bruns, v-SNARE actions during $\mathrm{Ca}(2+)$-triggered exocytosis, Cell 2007, 131, 351.

[171] F. Deák, O.-H. Shin, E. T. Kavalali, T. C. Südhof, Structural determinants of synaptobrevin 2 function in synaptic vesicle fusion, J Neurosci 2006, 26, 6668.

[172] A. D. Lam, P. Tryoen-Toth, B. Tsai, N. Vitale, E. L. Stuenkel, SNARE-catalyzed fusion events are regulated by Syntaxin1A-lipid interactions, Mol Biol Cell 2008, $19,485$.

[173] T. Weber, B. V. Zemelman, J. A. McNew, B. Westermann, M. Gmachl, F. Parlati, T. H. Söllner, J. E. Rothman, SNAREpins: Minimal Machinery for Membrane Fusion, Cell 1998, 92, 759.

[174] X. Chen, D. Araç, T.-M. Wang, C. J. Gilpin, J. Zimmerberg, J. Rizo, SNAREmediated lipid mixing depends on the physical state of the vesicles, Biophys $J \mathbf{2 0 0 6}$, 90, 2062.

[175] B. L. Sabatini, W. G. Regehr, Timing of neurotransmission at fast synapses in the mammalian brain, Nature 1996, 384, 170. 
[176] M. Ma, A. Paredes, D. Bong, Intra- and intermembrane pairwise molecular recognition between synthetic hydrogen-bonding phospholipids, J Am Chem Soc 2008, $130,14456$.

[177] S. A. Whitehead, C. D. McNitt, S. I. Mattern-Schain, A. J. Carr, S. Alam, V. V. Popik, M. D. Best, Artificial Membrane Fusion Triggered by Strain-Promoted Alkyne-Azide Cycloaddition, Bioconjug Chem 2017, 28, 923.

[178] Y.-H. M. Chan, B. van Lengerich, S. G. Boxer, Lipid-anchored DNA mediates vesicle fusion as observed by lipid and content mixing, Biointerphases 2008, 3, FA17.

[179] G. Stengel, L. Simonsson, R. A. Campbell, F. Höök, Determinants for membrane fusion induced by cholesterol-modified DNA zippers, J Phys Chem B 2008, 112, 8264.

[180] C. Böhler, P. E. Nielsen, L. E. Orgel, Template switching between PNA and RNA oligonucleotides, Nature 1995, 376, 578.

[181] P. Wittung, P. E. Nielsen, O. Buchardt, M. Egholm, B. Nordén, DNA-like double helix formed by peptide nucleic acid, Nature 1994, 368, 561.

[182] A. Rabe, P. M. G. Löffler, O. Ries, S. Vogel, Programmable fusion of liposomes mediated by lipidated PNA, Chem. Commun. 2017, 53, 11921.

[183] A. S. Lygina, K. Meyenberg, R. Jahn, U. Diederichsen, Transmembrane domain peptide/peptide nucleic acid hybrid as a model of a SNARE protein in vesicle fusion, Angew. Chem. Int. Ed. 2011, 50, 8597.

[184] J.-D. Wehland, A. S. Lygina, P. Kumar, S. Guha, B. E. Hubrich, R. Jahn, U. Diederichsen, Role of the transmembrane domain in SNARE protein mediated membrane fusion: peptide nucleic acid/peptide model systems, Mol. Biosyst. 2016, $12,2770$.

[185] B. E. Hubrich, P. Kumar, H. Neitz, M. Grunwald, T. Grothe, P. J. Walla, R. Jahn, U. Diederichsen, PNA Hybrid Sequences as Recognition Units in SNARE-ProteinMimicking Peptides, Angew. Chem. Int. Ed. 2018, 57, 14932.

[186] D. Weicherding, W. B. Davis, S. Hess, T. von Feilitzsch, M. E. Michel-Beyerle, U. Diederichsen, Femtosecond time-resolved guanine oxidation in acridine modified alanyl peptide nucleic acids, Bioorg Med Chem Lett 2004, 14, 1629.

[187] O. Berger, L. Adler-Abramovich, M. Levy-Sakin, A. Grunwald, Y. Liebes-Peer, M. Bachar, L. Buzhansky, E. Mossou, V. T. Forsyth, T. Schwartz et al., Light-emitting self-assembled peptide nucleic acids exhibit both stacking interactions and WatsonCrick base pairing, Nat Nanotechnol 2015, 10, 353. 
[188] Barbara Elisabeth Hubrich, Synthesis of model systems for SNARE mediated membrane fusion based on PNA/PNA base pair recognition, Cuvillier Verlag, Göttingen, 2018.

[189] M. Sadek, D. Berndt, D. Milovanovic, R. Jahn, U. Diederichsen, Distance Regulated Vesicle Fusion and Docking Mediated by $\beta$-Peptide Nucleic Acid SNARE Protein Analogues, Chembiochem 2016, 17, 479.

[190] H. R. Marsden, I. Tomatsu, A. Kros, Model systems for membrane fusion, Chem Soc Rev 2011, 40, 1572.

[191] H. Robson Marsden, A. V. Korobko, T. Zheng, J. Voskuhl, A. Kros, Controlled liposome fusion mediated by SNARE protein mimics, Biomater Sci 2013, 1, 1046.

[192] A. LUPAS, Coiled coils: new structures and new functions, Trends Biochem. Sci. 1996, 21,375 .

[193] K. M. Müller, K. M. Arndt, T. Alber in Methods in Enzymology, Elsevier, 2000, pp. 261-282.

[194] F. H. C. Crick, The packing of $\alpha$-helices: simple coiled-coils, Acta Cryst 1953, 6, 689.

[195] D. N. Woolfson in Advances in Protein Chemistry : Fibrous Proteins: Coiled-Coils, Collagen and Elastomers, Academic Press, 2005, pp. 79-112.

[196] D. A. Lindhout, J. R. Litowski, P. Mercier, R. S. Hodges, B. D. Sykes, NMR solution structure of a highly stable de novo heterodimeric coiled-coil, Biopolymers 2004, $75,367$.

[197] G. A. Daudey, H. R. Zope, J. Voskuhl, A. Kros, A. L. Boyle, Membrane-Fusogen Distance Is Critical for Efficient Coiled-Coil-Peptide-Mediated Liposome Fusion, Langmuir 2017, 33, 12443.

[198] F. Versluis, J. Voskuhl, B. van Kolck, H. Zope, M. Bremmer, T. Albregtse, A. Kros, In situ modification of plain liposomes with lipidated coiled coil forming peptides induces membrane fusion, $J$ Am Chem Soc 2013, 135, 8057.

[199] M. Rabe, C. Schwieger, H. R. Zope, F. Versluis, A. Kros, Membrane interactions of fusogenic coiled-coil peptides: implications for lipopeptide mediated vesicle fusion, Langmuir 2014, 30, 7724.

[200] G. A. Daudey, C. Schwieger, M. Rabe, A. Kros, Influence of Membrane-Fusogen Distance on the Secondary Structure of Fusogenic Coiled Coil Peptides, Langmuir 2019, 35, 5501.

[201] M. Rabe, H. R. Zope, A. Kros, Interplay between Lipid Interaction and Homocoiling of Membrane-Tethered Coiled-Coil Peptides, Langmuir 2015, 31, 9953.

[202] E. Strandberg, J. Killian, Snorkeling of lysine side chains in transmembrane helices: how easy can it get?, FEBS Lett 2003, 544, 69. 
[203] M. Rabe, C. Aisenbrey, K. Pluhackova, V. de Wert, A. L. Boyle, D. F. Bruggeman, S. A. Kirsch, R. A. Böckmann, A. Kros, J. Raap et al., A Coiled-Coil Peptide Shaping Lipid Bilayers upon Fusion, Biophys J 2016, 111, 2162.

[204] K. Pluhackova, T. A. Wassenaar, S. Kirsch, R. A. Böckmann, Spontaneous adsorption of coiled-coil model peptides $\mathrm{K}$ and $\mathrm{E}$ to a mixed lipid bilayer, $J$ Phys Chem B 2015, 119, 4396.

[205] S. Y. Woo, H. Lee, All-atom simulations and free-energy calculations of coiled-coil peptides with lipid bilayers: binding strength, structural transition, and effect on lipid dynamics, Sci Rep 2016, 6, 22299.

[206] Karsten Meyenberg, Synthese und Untersuchungen von peptidischen Modellsystemen für SNARE-induzierte Membranfusion, Books on Demand, Norderstedt, 2011.

[207] J.-D. Wehland, Hybrids of SNARE Transmembrane Domains and Aritificial Recognition Motifs as Membrane Fusion Inducing Model Peptides, PhD thesis, Georg-August-Universität, Göttingen, 2017.

[208] A. T. Brunger, D. J. Cipriano, J. Diao, Towards reconstitution of membrane fusion mediated by SNAREs and other synaptic proteins, Crit Rev Biochem Mol Biol 2015, $50,231$.

[209] J. W. Kuhlmann, M. Junius, U. Diederichsen, C. Steinem, SNARE-Mediated Single-Vesicle Fusion Events with Supported and Freestanding Lipid Membranes, Biophys J 2017, 112, 2348.

[210] D. K. Struck, D. Hoekstra, R. E. Pagano, Use of resonance energy transfer to monitor membrane fusion, Biochemistry 1981, 20, 4093.

[211] T. Forster, Energiewanderung und Fluoreszenz, Naturwissenschaften 1946, 33, 166.

[212] B. T. Bajar, E. S. Wang, S. Zhang, M. Z. Lin, J. Chu, A Guide to Fluorescent Protein FRET Pairs, Sensors (Basel) 2016, 16.

[213] L. M. S. Loura, M. Prieto, FRET in Membrane Biophysics: An Overview, Front Physiol 2011, 2, 82.

[214] V. Trivedi, C. Yu, B. Veeramuthu, S. Francis, D. Chang, Fusion induced aggregation of model vesicles studied by dynamic and static light scattering, Chem Phys Lipids 2000, 107, 99.

[215] S. Castorph, S. Schwarz Henriques, M. Holt, D. Riedel, R. Jahn, T. Salditt, Synaptic vesicles studied by dynamic light scattering, Eur Phys J E Soft Matter 2011, 34, 63.

[216] A.-K. Awizio, F. Onofri, F. Benfenati, E. Bonaccurso, Influence of synapsin I on synaptic vesicles: an analysis by force-volume mode of the atomic force microscope and dynamic light scattering, Biophys $J$ 2007, 93, 1051. 
[217] N. Moskowitz, S. Puszkin, W. Schook, Characterization of brain synaptic vesicle phospholipase A2 activity and its modulation by calmodulin, prostaglandin E2, prostaglandin F2 alpha, cyclic AMP, and ATP, J Neurochem 1983, 41, 1576.

[218] J. Stetefeld, S. A. McKenna, T. R. Patel, Dynamic light scattering: a practical guide and applications in biomedical sciences, Biophys Rev 2016, 8, 409.

[219] A. Schirmacher, Modification of transmembrane peptides to probe SNARE-induced membrane fusion and cross-presentation of membrane-buried epitopes, GeorgAugust-Universität, Göttingen, 2020.

[220] H. Chao, D. L. Bautista, J. Litowski, R. T. Irvin, R. S. Hodges, Use of a heterodimeric coiled-coil system for biosensor application and affinity purification, J. chromatogr., B, Biomed. sci. appl. 1998, 715, 307.

[221] H. Chao, M. E. Houston, S. Grothe, C. M. Kay, M. O'Connor-McCourt, R. T. Irvin, R. S. Hodges, Kinetic Study on the Formation of a de Novo Designed Heterodimeric Coiled-Coil: Use of Surface Plasmon Resonance To Monitor the Association and Dissociation of Polypeptide Chains $\uparrow$, Biochemistry 1996, 35, 12175.

[222] B. Tripet, L. Yu, D. L. Bautista, W. Y. Wong, R. T. Irvin, R. S. Hodges, Engineering a de novo -designed coiled-coil heterodimerization domain for the rapid detection, purification and characterization of recombinantly expressed peptides and proteins, Protein Eng Des Sel 1996, 9, 1029.

[223] R. B. Merrifield, Solid Phase Peptide Synthesis. I. The Synthesis of a Tetrapeptide, J Am Chem Soc 1963, 85, 2149.

[224] J. M. Collins, S. K. Singh, "Use of Excess Carbodiimide for Peptide Synthesis at Elevated Temperatures", 2018, US20180066013A1.

[225] B. Apostolovic, H.-A. Klok, pH-sensitivity of the E3/K3 heterodimeric coiled coil, Biomacromolecules 2008, 9, 3173.

[226] P. Padmanabhan, A. T. Bademosi, R. Kasula, E. Lauwers, P. Verstreken, F. A. Meunier, Need for speed: Super-resolving the dynamic nanoclustering of syntaxin1 at exocytic fusion sites, Neuropharmacology 2020, 169, 107554.

[227] C. Rickman, C. N. Medine, A. R. Dun, D. J. Moulton, O. Mandula, N. D. Halemani, S. O. Rizzoli, L. H. Chamberlain, R. R. Duncan, t-SNARE protein conformations patterned by the lipid microenvironment, J Biol Chem 2010, 285, 13535.

[228] J. J. Sieber, K. I. Willig, R. Heintzmann, S. W. Hell, T. Lang, The SNARE motif is essential for the formation of syntaxin clusters in the plasma membrane, Biophys $J$ 2006, 90, 2843.

[229] A. Ullrich, M. A. Böhme, J. Schöneberg, H. Depner, S. J. Sigrist, F. Noé, Dynamical Organization of Syntaxin-1A at the Presynaptic Active Zone, PLoS Comput Biol 2015, 11, e1004407. 
[230] B. L. Bray, Large-scale manufacture of peptide therapeutics by chemical synthesis, Nat Rev Drug Discov 2003, 2, 587.

[231] K. Kachel, E. Asuncion-Punzalan, E. London, The location of fluorescence probes with charged groups in model membranes, Biochim Biophys Acta Biomembr 1998, $1374,63$.

[232] D. E. Wolf, A. P. Winiski, A. E. Ting, K. M. Bocian, R. E. Pagano, Determination of the transbilayer distribution of fluorescent lipid analogues by nonradiative fluorescence resonance energy transfer, Biochemistry 1992, 31, 2865.

[233] S. Fery-Forgues, J.-P. Fayet, A. Lopez, Drastic changes in the fluorescence properties of NBD probes with the polarity of the medium: involvement of a TICT state?, J. Photochem. Photobiol. A 1993, 70, 229.

[234] A. Chattopadhyay, E. London, Spectroscopic and ionization properties of N-(7nitrobenz-2-oxa-1,3-diazol-4-yl)-labeled lipids in model membranes, Biochim Biophys Acta Biomembr 1988, 938, 24.

[235] Fehmke Reichardt, Untersuchung des Einflusses der Linker-Region von CoiledCoil SNARE-Analoga auf Vesikelfusion, Bachelor Thesis, Georg-AugustUniversität, Göttingen, 2020.

[236] J. A. McNew, T. Weber, D. M. Engelman, T. H. Söllner, J. E. Rothman, The Length of the Flexible SNAREpin Juxtamembrane Region Is a Critical Determinant of SNARE-Dependent Fusion, Mol. Cell 1999, 4, 415.

[237] S. S. Rathore, Y. Liu, H. Yu, C. Wan, M. Lee, Q. Yin, M. H. B. Stowell, J. Shen, Intracellular Vesicle Fusion Requires a Membrane-Destabilizing Peptide Located at the Juxtamembrane Region of the v-SNARE, Cell Rep 2019, 29, 4583-4592.e3.

[238] C. M. DeMill, X. Qiu, M. Kisiel, A. Bolotta, B. A. Stewart, Investigation of the juxtamembrane region of neuronal-Synaptobrevin in synaptic transmission at the Drosophila neuromuscular junction, J Neurophysiol 2014, 112, 1356.

[239] Y. Lai, U. B. Choi, J. Leitz, H. J. Rhee, C. Lee, B. Altas, M. Zhao, R. A. Pfuetzner, A. L. Wang, N. Brose et al., Molecular Mechanisms of Synaptic Vesicle Priming by Munc13 and Munc18, Neuron 2017, 95, 591-607.e10.

[240] D.-H. Kweon, C. S. Kim, Y.-K. Shin, The membrane-dipped neuronal SNARE complex: a site-directed spin labeling electron paramagnetic resonance study, Biochemistry 2002, 41, 9264.

[241] G. van den Bogaart, K. Meyenberg, H. J. Risselada, H. Amin, K. I. Willig, B. E. Hubrich, M. Dier, S. W. Hell, H. Grubmüller, U. Diederichsen et al., Membrane protein sequestering by ionic protein-lipid interactions, Nature 2011, 479, 552. 
[242] D. Greitzer-Antes, N. Barak-Broner, S. Berlin, Y. Oron, D. Chikvashvili, I. Lotan, Tracking $\mathrm{Ca} 2+-$ dependent and $\mathrm{Ca} 2+$-independent conformational transitions in syntaxin 1A during exocytosis in neuroendocrine cells, J Cell Sci 2013, 126, 2914.

[243] S. Barg, M. K. Knowles, X. Chen, M. Midorikawa, W. Almers, Syntaxin clusters assemble reversibly at sites of secretory granules in live cells, Proc Natl Acad Sci $U$ $S$ A 2010, 107, 20804.

[244] A. T. Bademosi, E. Lauwers, P. Padmanabhan, L. Odierna, Y. J. Chai, A. Papadopulos, G. J. Goodhill, P. Verstreken, B. van Swinderen, F. A. Meunier, In vivo single-molecule imaging of syntaxin1A reveals polyphosphoinositide- and activity-dependent trapping in presynaptic nanoclusters, Nat Commun 2017, 8, 13660.

[245] D. H. Murray, L. K. Tamm, Molecular mechanism of cholesterol- and polyphosphoinositide-mediated syntaxin clustering, Biochemistry 2011, 50, 9014.

[246] T. F. J. Martin, PI(4,5) $\mathrm{P}_{2}$-binding effector proteins for vesicle exocytosis, Biochim Biophys Acta 2015, 1851, 785.

[247] F. Huang, W. M. Nau, A conformational flexibility scale for amino acids in peptides, Angew. Chem. Int. Ed. 2003, 42, 2269.

[248] E. E. Oude Blenke, J. van den Dikkenberg, B. van Kolck, A. Kros, E. Mastrobattista, Coiled coil interactions for the targeting of liposomes for nucleic acid delivery, Nanoscale 2016, 8, 8955.

[249] G. van den Bogaart, T. Lang, R. Jahn, Microdomains of SNARE proteins in the plasma membrane, Curr Top Membr 2013, 72, 193.

[250] R. L. Thurlkill, G. R. Grimsley, J. M. Scholtz, C. N. Pace, pK values of the ionizable groups of proteins, Protein Sci 2006, 15, 1214.

[251] L. J. G. W. van Wilderen, C. Neumann, A. Rodrigues-Correia, D. Kern-Michler, N. Mielke, M. Reinfelds, A. Heckel, J. Bredenbeck, Picosecond activation of the DEACM photocage unravelled by VIS-pump-IR-probe spectroscopy, Phys Chem Chem Phys 2017, 19, 6487.

[252] V. Hagen, S. Frings, B. Wiesner, S. Helm, U. B. Kaupp, J. Bendig, 7(Dialkylamino)coumarin-4-ylmethyl-Caged Compounds as Ultrafast and Effective Long-Wavelength Phototriggers of 8-Bromo-Substituted Cyclic Nucleotides, Chembiochem 2003, 4, 434.

[253] J. Luo, R. Uprety, Y. Naro, C. Chou, D. P. Nguyen, J. W. Chin, A. Deiters, Genetically encoded optochemical probes for simultaneous fluorescence reporting and light activation of protein function with two-photon excitation, $J$ Am Chem Soc 2014, 136, 15551. 
[254] X. Zhang, W. Xi, C. Wang, M. Podgórski, C. N. Bowman, Visible-Light-Initiated Thiol-Michael Addition Polymerizations with Coumarin-Based Photobase Generators: Another Photoclick Reaction Strategy, ACS Macro Lett 2016, 5, 229.

[255] L.-M. Herzig, I. Elamri, H. Schwalbe, J. Wachtveitl, Light-induced antibiotic release from a coumarin-caged compound on the ultrafast timescale, Phys Chem Chem Phys 2017, 19, 14835.

[256] A. V. Pinheiro, P. Baptista, J. C. Lima, Light activation of transcription: photocaging of nucleotides for control over RNA polymerization, Nucleic Acids Res 2008, 36, e90.

[257] J. Bai, W. C. Tucker, E. R. Chapman, PIP2 increases the speed of response of synaptotagmin and steers its membrane-penetration activity toward the plasma membrane, Nat Struct Mol Biol 2004, 11, 36.

[258] T. Liang, L. Xie, C. Chao, Y. Kang, X. Lin, T. Qin, H. Xie, Z.-P. Feng, H. Y. Gaisano, Phosphatidylinositol 4,5-biphosphate (PIP2) modulates interaction of syntaxin-1A with sulfonylurea receptor 1 to regulate pancreatic $\beta$-cell ATPsensitive potassium channels, J Biol Chem 2014, 289, 6028.

[259] P. M. Kasson, V. S. Pande, Control of membrane fusion mechanism by lipid composition: predictions from ensemble molecular dynamics, PLoS Comput Biol 2007, 3, e220.

[260] G. van Meer, J. Davoust, K. Simons, Parameters affecting low-pH-mediated fusion of liposomes with the plasma membrane of cells infected with influenza virus, Biochemistry 1985, 24, 3593.

[261] K. Emoto, M. Umeda, An essential role for a membrane lipid in cytokinesis. Regulation of contractile ring disassembly by redistribution of phosphatidylethanolamine, J Cell Biol 2000, 149, 1215.

[262] L. H. Chamberlain, R. D. Burgoyne, G. W. Gould, SNARE proteins are highly enriched in lipid rafts in PC12 cells: implications for the spatial control of exocytosis, Proc Natl Acad Sci U S A 2001, 98, 5619.

[263] J. Zhang, R. Xue, W.-Y. Ong, P. Chen, Roles of cholesterol in vesicle fusion and motion, Biophys J 2009, 97, 1371.

[264] S. Takamori, M. Holt, K. Stenius, E. A. Lemke, M. Grønborg, D. Riedel, H. Urlaub, S. Schenck, B. Brügger, P. Ringler et al., Molecular anatomy of a trafficking organelle, Cell 2006, 127, 831.

[265] M. K. Domanska, V. Kiessling, L. K. Tamm, Docking and fast fusion of synaptobrevin vesicles depends on the lipid compositions of the vesicle and the acceptor SNARE complex-containing target membrane, Biophys $J$ 2010, 99, 2936. 
[266] M. E. Haque, T. J. McIntosh, B. R. Lentz, Influence of lipid composition on physical properties and peg-mediated fusion of curved and uncurved model membrane vesicles: "nature's own" fusogenic lipid bilayer, Biochemistry 2001, 40, 4340.

[267] A. Honigmann, G. van den Bogaart, E. Iraheta, H. J. Risselada, D. Milovanovic, V. Mueller, S. Müllar, U. Diederichsen, D. Fasshauer, H. Grubmüller et al., Phosphatidylinositol 4,5-bisphosphate clusters act as molecular beacons for vesicle recruitment, Nat Struct Mol Biol 2013, 20, 679.

[268] S. McLaughlin, J. Wang, A. Gambhir, D. Murray, PIP(2) and proteins: interactions, organization, and information flow, Annu Rev Biophys Biomol Struct 2002, 31, 151.

[269] J. Wang, A. Gambhir, G. Hangyás-Mihályné, D. Murray, U. Golebiewska, S. McLaughlin, Lateral sequestration of phosphatidylinositol 4,5-bisphosphate by the basic effector domain of myristoylated alanine-rich $\mathrm{C}$ kinase substrate is due to nonspecific electrostatic interactions, J. Biol. Chem. 2002, 277, 34401.

[270] J. R. Lakowicz, Principles of Fluorescence Spectroscopy, Springer US, Boston, MA, 1999.

[271] M. R. Eftink, Fluorescence techniques for studying protein structure, Methods Biochem Anal 1991, 35, 127.

[272] P. Mühlenbrock, K. Herwig, L. Vuong, I. Mey, C. Steinem, Fusion Pore Formation Observed during SNARE-Mediated Vesicle Fusion with Pore-Spanning Membranes, Biophys J 2020, 119, 151.

[273] R. Hubrich, Y. Park, I. Mey, R. Jahn, C. Steinem, SNARE-Mediated Fusion of Single Chromaffin Granules with Pore-Spanning Membranes, Biophys J 2019, 116, 308.

[274] S. C. Gill, P. H. von Hippel, Calculation of protein extinction coefficients from amino acid sequence data, Anal Biochem 1989, 182, 319.

[275] M. J. Hope, M. B. Bally, G. Webb, P. R. Cullis, Production of large unilamellar vesicles by a rapid extrusion procedure. Characterization of size distribution, trapped volume and ability to maintain a membrane potential, Biochim Biophys Acta Biomembr 1985, 812, 55. 


\section{Acknowledgments}

I would like to thank my supervisor Prof. Dr. Ulf Diederichsen for giving me the opportunity to work in an interesting scientific field in his group. I was granted freedom in my scientific research as well as in my self-development and was supported by you with trust in my capabilities.

I am grateful for my second supervisor Prof. Dr. Claudia Steinem for helpful scientific discussions and advices. Particularly all SFB 803 related colloquia and conferences were well organized by you and your team and I am thankful to be a part of this scientific group.

Furthermore, I would like to thank Prof. Dr. Kai Tittmann, Prof. Dr. Michael Meinecke, Prof. Dr. Jörg Enderlein and Dr. Holm Frauendorf for being part of my examination board. Moreover, I would like to thank Dr. Frauendorf and his team of the MS analytical department for swiftly measuring all my peptide mass spectra.

I thank the whole Diederichsen group for a pleasant time during the last four years, especially Lab 102. I really enjoyed sharing my lab with Brigitte Worbs who stands out with her admirable work ethic and was always an enjoyable conversational partner. Dr. Barbara Hubrich, Dr. Jan-Dirk Wehland, Robert Schrimacher and Viktoria Mrden Debono with whom I had a great time in lab 102 as well.

The sessions at the foosball table were essential for our good group dynamic and were a bright spot in dark times during my $\mathrm{PhD}$ time. For this I want to thank Pirajeev Selvachandran, Benedikt Kugler, Tobias Schmidt, Dr. Matthias Krull and Mathis Rink.

I am very grateful for Martin Kloos, Pirajeev Selvachandran, Clara Bosbach and Tobias Schmidt for reading and correcting my work.

I thank my bachelor student Fehmke Reichardt, who performed exceptionally well in all tasks she did during her bachelor thesis and her uplifting spirit during our time together.

I would also like to thank Jun.-Prof. Dr. Franziska Thomas and her work group for support in scientific problems. Especially Vanessa Reusche who helped me in the BIN with the peptide synthesizer.

Addionally, I want to thank Angela Heinemann for handling all administrational matters. 
I would also like to thank my friends and fellow chemistry students Valentin Müller, Marcus Thater and Jerome Spyra who fought valiantly with me during the bachelor, master and $\mathrm{PhD}$ time.

I am very grateful for my wife Anika Groth, who supported me throughout my bachelor, master and $\mathrm{PhD}$ time in every way imaginable. Without you there would not be much happiness in my life. I am very lucky that you are by my side for the rest of my life.

Ich bin sehr dankbar dafür, dass meine Eltern und mein Bruder mich mein Leben lang begleitet und immer unterstützt haben. Ihr habt immer an mich geglaubt und habt mich nie im Stich gelassen 